Lehrstuhl für Apparate- und Anlagenbau

Experimentelle Spannungsanalyse

der Technischen Universität München

\title{
Dreidimensionale numerische Simulation strömungsinduzierter Rohrbündelschwingungen
}

\author{
Michael Fischer \\ Vollständiger Abdruck der von der Fakultät für Maschinenwesen \\ der Technischen Universität München zur Erlangung \\ des akademischen Grades eines \\ Doktor-Ingenieurs \\ genehmigten Dissertation.
}

Vorsitzender: Univ.-Prof. Dr.-Ing., Dr.-Ing. habil. J. Stichlmair

Prüfer der Dissertation:

1. Univ.-Prof. Dr.-Ing. K. Strohmeier

2. Univ.-Prof. Dr.-Ing., Dr.-Ing. habil. R. Friedrich

Die Dissertation wurde am 26.09.2001 bei der Technischen Universität München eingereicht und durch die Fakultät für Maschinenwesen am 21.11.2001 angenommen. 
Die vorliegende Arbeit wurde im Internet auf dem Server der Bibliothek der Technischen Universität München veröffentlicht unter der Adresse

http://tumb1.biblio.tu-muenchen.de/publ/diss/

Druck: Eigenverlag 
Die vorliegende Arbeit entstand während meiner Zeit als Stipendiat und wissenschaftlicher Assistent am Lehrstuhl für Apparate- und Anlagenbau - Experimentelle Spannungsanalyse der Technischen Universität München. Meinem Doktorvater Ordinarius Univ.-Prof. Dr.-Ing. Klaus Strohmeier danke ich für die finanzielle und moralische Unterstützung des Vorhabens, die Beschaffung einer Reihe von Literaturstellen, die großen Freiheiten bei der Bearbeitung des Dissertationsthemas, der Organisation des alljährlich in Übersee stattfindenen „Symposium on Emerging Technologies in Fluids, Structures and Fluid/Structure Interaction“ im Rahmen der ASME-Konferenzen und allen anfallenden Lehrstuhlaufgaben, das stets gute Arbeitsverhältnis und die Übernahme der Korrektur. Herrn Univ.-Prof. Dr.-Ing., Dr.-Ing. habil. Rainer Friedrich danke ich für die Übernahme des Korreferates, Herrn Univ.-Prof. Dr.-Ing., Dr.Ing. habil. Johann Stichlmair für die Übernahme des Prüfungsvorsitzes.

Ohne die große Kollegialität am Lehrstuhl wäre die Anfertigung der vorliegenden Arbeit deutlich schwerer gefallen, hierfür danke ich allen Mitarbeitern des Lehrstuhls für Apparate- und Anlagenbau. Großer Dank gilt meinem direkten Vorgänger, Herrn Dr.-Ing. Volker Kassera, für die reichhaltigen Vorarbeiten auf meinem Gebiet und die große Anzahl an fachlichen Diskussionen. Außerdem war er es, der mich als studierten Luft- und Raumfahrttechniker hinsichtlich des Gebiets des Apparatebaus erst ,auf den Geschmack“ gebracht und mich in den Lehrstuhl eingeführt hat. Weiter danke ich meinem ehemaligen Kollegen Herrn Dr.-Ing. Reinhold Hölzl für die fachliche Unterstützung bei der Anwendung des hydraulischen Aufweitens für die Anfertigung meiner Experimentierrohrbündel und die fachliche Diskussion aller Probleme der Berechnung im Apparatebau. Meinem ehemaligen Kollegen Herrn Dr.-Ing. Edgar Merkl danke ich für die zügige und professionelle Abwicklung des Umzugs unseres Lehrstuhl in die neuen Räumlichkeiten in Garching, wodurch der Prüfstand rechtzeitig für die Messungen fertig werden konnte. Meinem Kollegen Dipl.-Ing. (Univ.) Jürgen Deininger danke ich für seine Unterstützung bei der Berechnung der Kontaktsteifigkeiten mit der Methode der Finiten Elemente und der Durchführung der zugehörigen Druckversuche. Außerdem ist allen Kollegen zu danken, die im Verlauf der Jahre das Rechnernetz und die Arbeitsplatzrechner des Lehrstuhls betreut haben. 
Allen an der Arbeit beteiligten Studenten danke ich für ihren unermüdlichen Einsatz als Semesterarbeiter, Diplomarbeiter oder studentische Hilfskraft. Besonders bedanken möchte ich mich bei Mathias Birkner für seine Programmierarbeiten, Dieter Dragon für seine konstruktiven und numerischen Arbeiten, Jennifer Stolz für ihre experimentellen Arbeiten und Auswertungen, sowie Ludwig Bauer für eine Unzahl von Messungen und seine Programmierarbeiten.

Für die Unterstützung bei der Vorbereitung des Folgeprojekts danke ich Ulrike Haber für ihre umfangreichen Konstruktionstätigkeiten, sowie Mirjam Kretschmar und Theo Paul Kaam für ihre numerischen Arbeiten.

Großer Dank gilt den Angehörigen der lehrstuhleigenen Werkstatt, allen voran unserem ehemaligen Werkstattmeister Herrn Anton Viehweider für seine fortwährenden Bemühungen, den Prüfstand vorwärts zu bringen. Außerdem danke ich Herrn Herbert Eppert für seine unermüdlichen Arbeiten zur Aufrechterhaltung der Meßtechnik, sowie Herrn Helmut Herzig und Herrn Josef Geissinger für die fachgerechte und hochqualitative Durchführung aller Holz- und Metallarbeiten. Frau Erika Jäger danke ich für die mit großer Sorgfalt angefertigten Fotodokumentationsarbeiten.

Nicht zuletzt gilt schließlich mein besonderer Dank Frau Luise Fuchs, die die große Mühe des Korrekturlesens des Manuskriptes auf sich nahm. 


\section{Inhalt}

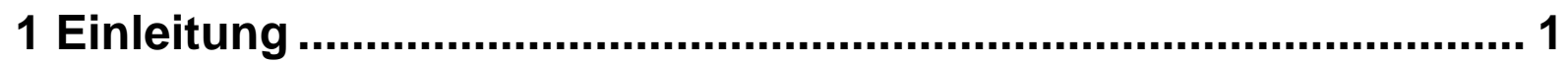

1.1 Funktionsprinzip, wirtschaftliche Aspekte und Schadensfälle ................. 1

1.2 Erregermechanismen .......................................................................................2

1.3 Bisherige Absicherung gegen Rohrbündelschwingungen .......................... 4

1.4 Literaturübersicht: Numerische Arbeiten................................................6

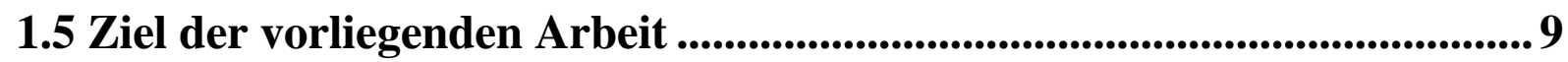

1.6 Gliederung der Arbeit.......................................................................11

2 Das numerische Simulationsverfahren...................................... 13

2.1 Simulation des Strömungsfeldes im Mantelraum ...................................13

2.1.1 Strömungsmechanische Grundgleichungen ......................................... 13

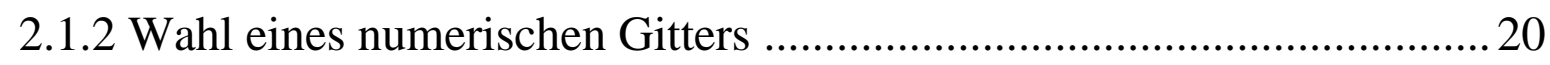

2.1.3 Diskretisierung der strömungsmechanischen Grundgleichungen .........21

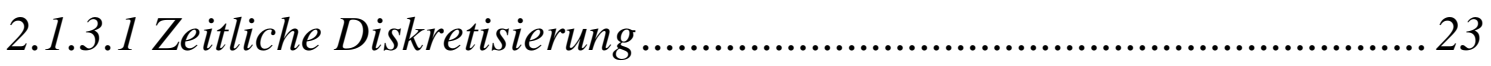

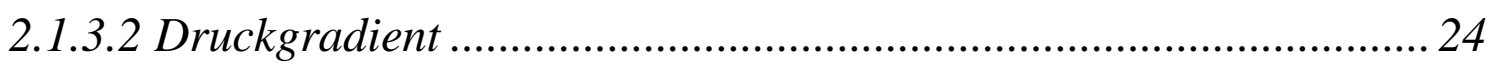

2.1.3.3 Gemischte Konvektions/Diffusions-Terme ................................... 24

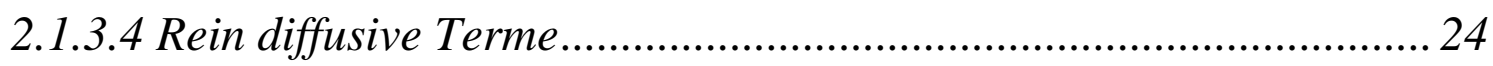

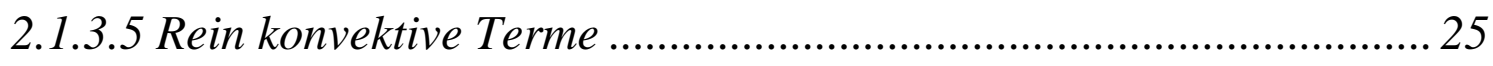

2.1.3.6 Zusammenfassung der Impulsgleichung ........................................26

2.1.3.7 Ergänzung um weitere konvektive Diskretisierungsschemata .......27

2.1.4 Lösung des Gleichungssystems ..........................................................29

2.1.4.1 Die Berechnung des Druckfeldes................................................... 29

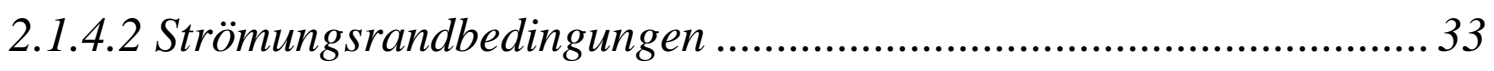

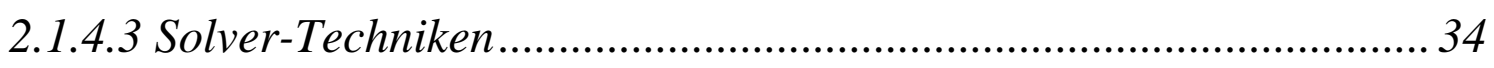

2.1.4.4 Ablauf der strömungsmechanischen Berechnung .......................... 36

2.1.5 Testberechnungen: Kräfte auf einen umströmten Einzelzylinder ..........36

2.1.6 Alternative Diskretisierungstechniken ................................................. 40

2.1.6.1 Die Finite Analytische Methode .................................................... 40

2.1.6.2 Die Finite Elemente Methode ........................................................ 45

2.1.7 Analytische Behandlung der Spaltströmung ........................................ 46

2.2 Simulation der strukturmechanischen Rohrbündeldynamik..................50

2.2.1 Strukturmechanische Grundgleichungen ........................................50

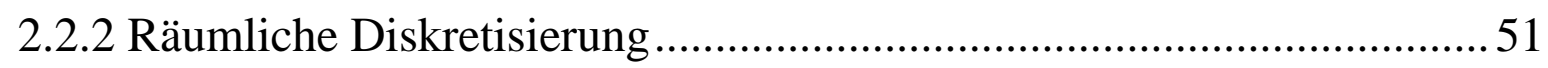

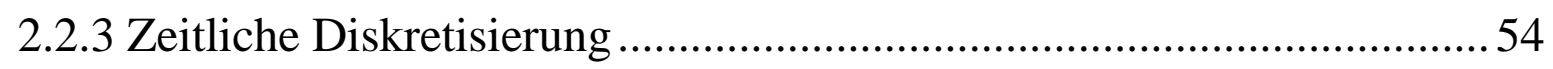


2.2.4 Zusätzliche Randbedingungen an den Leitblechen ..............................55

2.2.4.1 Modifikation der Steifigkeitsmatrix an der Lagerstelle ..................56

2.2.4.2 Mechanische Zusatzkräfte infolge der Reibstöße im Leitblech ...... 57

2.2.4.3 Filmkräfte aufgrund von Spaltströmung .......................................60

2.2.4.4 Zusammenfassung und Beispielrechnung Kontaktmodell .............. 62

2.2.5 Iterative Lösung des Gleichungssystems .............................................63

2.2.6 Überprüfung der Modellantwort mit analytischen Formeln ..................65

2.3 Kopplung von Strömung und Struktur .........................................................66

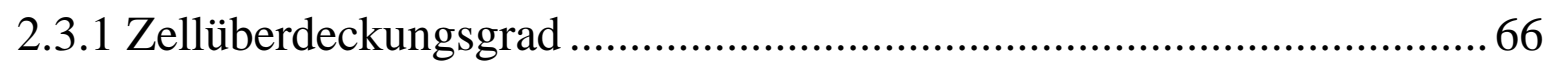

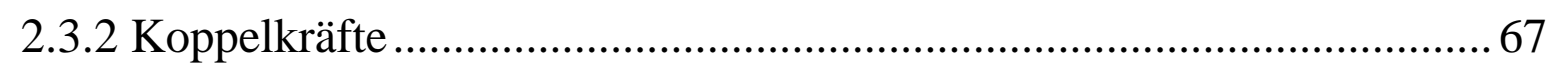

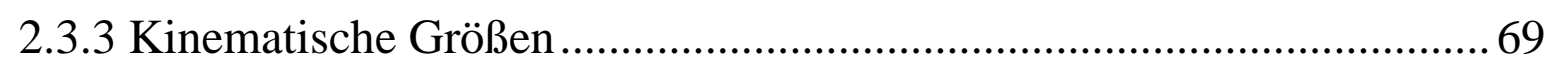

2.3.4 Verbesserung des Druckkorrekturverfahrens .....................................69

2.3.5 Ablauf eines fluid-struktur-gekoppelten Zeitschritts ........................... 70

2.3.6 Berechnungsbeispiele: Schwingender Einzelzylinder in einem Kanal .71

2.4 Stabile numerische Parameter ................................................................ 71

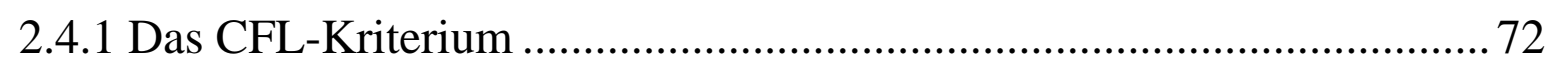

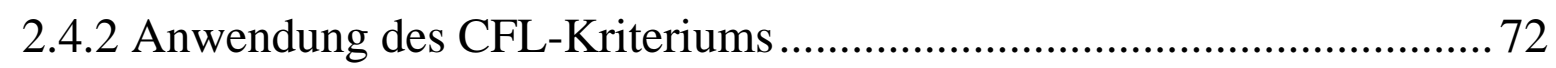

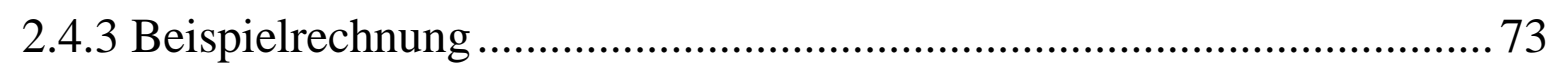

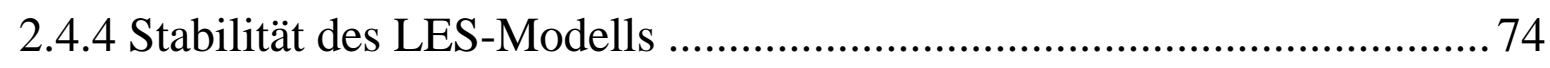

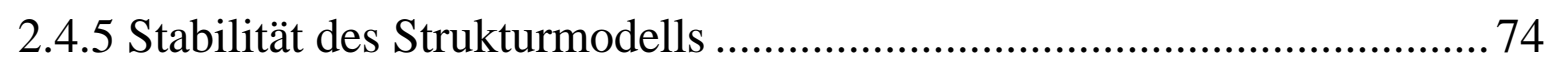

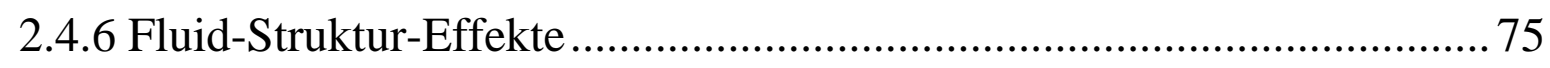

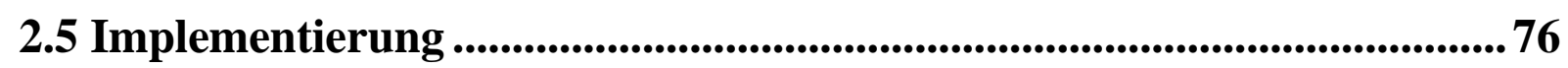

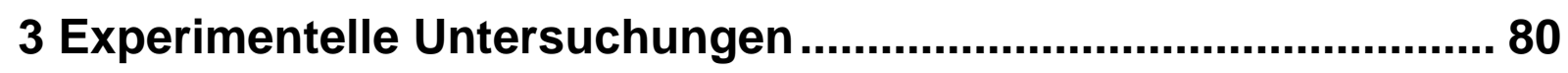

3.1 Beschreibung der Versuchseinrichtung .......................................................80

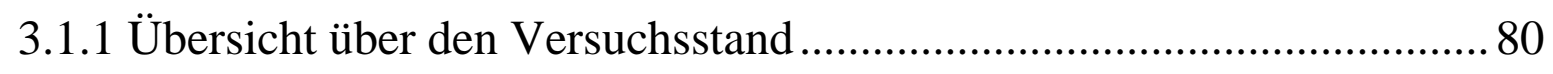

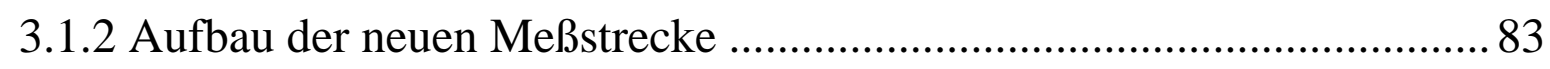

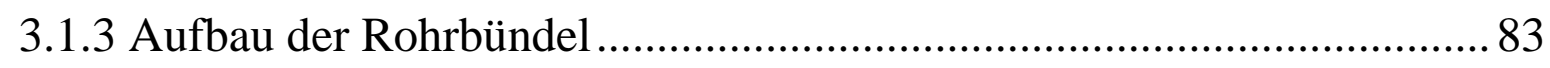

3.1.4 Zielmeßgröße und verwendete Meßtechnik ...................................... 90

3.2 Versuchsprogramm............................................................................94

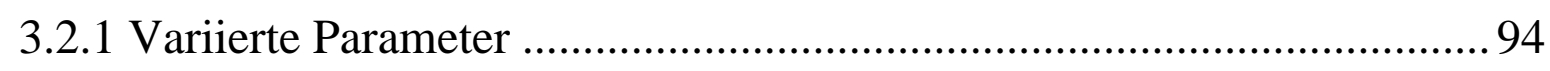

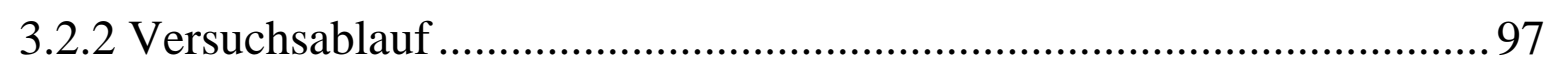

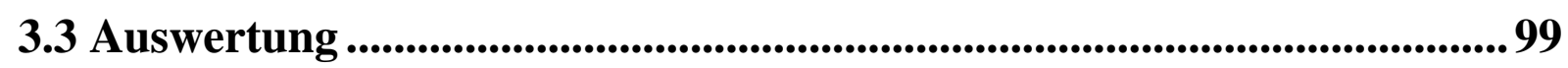

3.3.1 RMS-Werte in Abhängigkeit der Rohrlängskoordinate ........................ 99

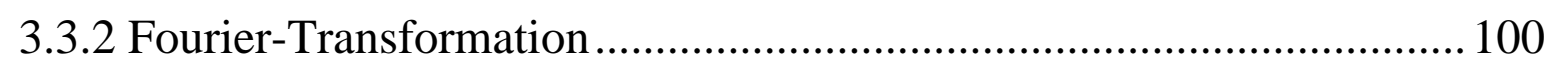

3.3.3 Rohrcharakteristische Fouriertransformierte ..................................... 101 
3.3.4 Analytische Identifikation der Schwingungsmoden ........................... 102

3.4 Feststellbare Schwingungsphänomene .....................................................108

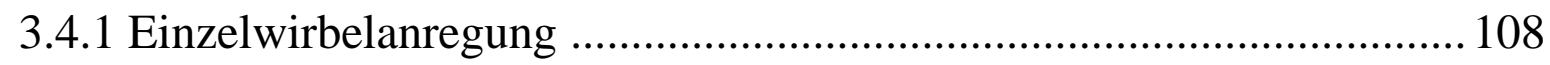

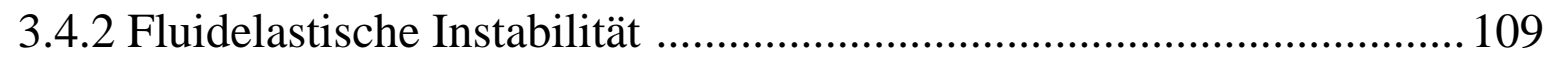

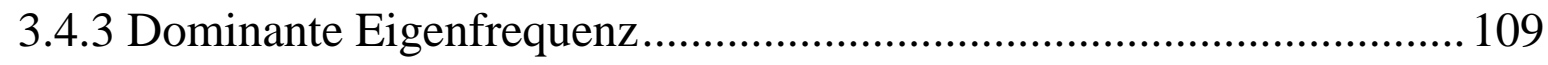

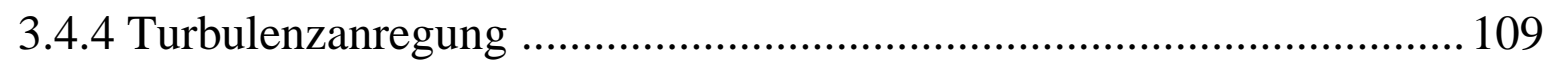

3.5 Validierung der Messungen anhand theoretischer Überlegungen .......110

3.5.1 Vergleich bei großen Teilungsverhältnissen ....................................... 110

3.5.2 Kritik am Konzept der hydrodynamischen Zusatzmassen ................. 113

3.5.3 Erweiterung des Theorievergleichs auf kleine Teilungsverhältnisse .. 115

4 Berechnungsbeispiele........................................................... 118

4.1 Relevante Schadensmechanismen .............................................................. 118

4.2 Bisherige halbempirische Absicherung...............................................118

4.2.1 Bestimmung der Rohreigenfrequenzen.............................................. 118

4.2.2 Bestimmung des Strömungsfeldes im Mantelraum ............................ 120

4.2.3 Anregung durch Einzelwirbelablösung ............................................ 122

4.2.4 Halbempirische Kriterien gegen fluidelastische Instabilität ............... 125

4.3 Zusammenfassung und Methodenvergleich ...........................................126

4.4 Ergebnisse aus numerischer Simulation ................................................127

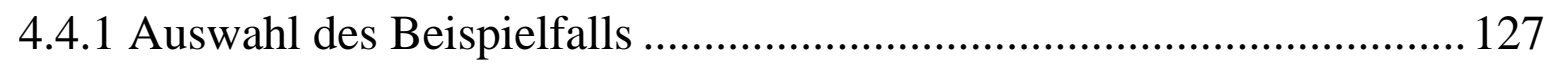

4.4.2 Vorgehen bei der Berechnung und Berechnungsparameter ................ 128

4.4.3 Beschreibung der Simulationsergebnisse ............................................ 129

5 Zusammenfassung und Ausblick.............................................. 134

5.1 Zusammenfassung der Arbeitsinhalte ...................................................134

5.2 Weiterentwicklungen gegenüber Kassera ...........................................135

5.3 Vorteile der Simulation gegenüber bisherigen Verfahren...................... 139

5.4 Vorteile gegenüber kommerziellen Simulationscodes ............................140

5.5 Mögliche zukünftige Entwicklungen ............................................................. 141

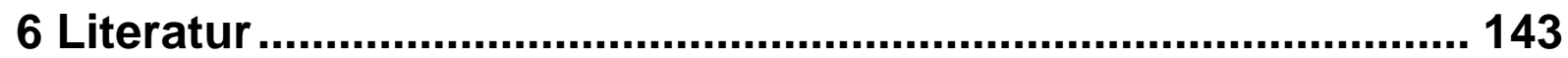

7 Anhang: Farbabbildungen ....................................................... 163 


\section{Nomenklatur}

\section{Lateinische Symbole}

A

$a$

B

Hilfsgröße für Definitionen

Koeffizienten in numerischen Gleichungen

Hilfsgröße für Definitionen

Massenerhaltungsfehler einer Strömungszelle

Smagorinsky-Konstante im dynamischen LES-Modell

Smagorinsky-Konstante im Standard-LES-Modell

Dämpfungsmatrix im Strukturmodell

Freie Konstanten bei Lösung von Differentialgleichungen

Auftriebsbeiwert

Courant-Friedrichs-Levy-Zahl

Widerstandsbeiwert

Rohraußendurchmesser

Rohrinnendurchmesser

Ersatzhülsenaußendurchmesser beim hydraulischen Aufweiten

Elastizitätsmodul

Rohrwandstärke

Axialkraft am Rohr bzw. Strömungskraft auf Zylinder

Kontaktkraft an einem Rohr in Stoßrichtung

Kontaktkraft an einem Rohr in Tangentialrichtung

Rohreigenfrequenz der $i$-ten Eigenform

Fehlergröße

Flächenträgheitsmoment

Empirischer Stabilitätsfaktor im Connors-Kriterium

Steifigkeitsmatrix im Strukturmodell

Biegesteifigkeitsfaktor im numerischen Strukturmodell

Axialsteifigkeitsfaktor im numerischen Strukturmodell

Kinetische Energie turbulenter Fluktuationen je Masseneinheit

Koeffizient der hydraulischen Zusatzmassen

Kontaktsteifigkeit nach Finite-Elemente-Lösung

Kontaktsteifigkeit an einem Rohr in Stoßrichtung

Stützweite eines Rohrabschnitts

Freie Gesamtrohrlänge 
$L_{i} \quad$ Leckagefaktoren

$L_{i j} \quad$ test-window-Spannungstensor

$M \quad$ Biegemoment

M Massenmatrix im Strukturmodell

$M_{i j} \quad$ Kontrahierender Tensor im Lilly-Germano-LES-Turbulenzmodell

$m \quad$ Rohrmasse pro Rohrlänge

$n \quad$ Anzahl der Meßpunkte einer Meßreihe

$n_{x} \quad$ Knotenzahl im numerischen Strukturmodell

$\Delta n \quad$ Laterale Rohreindrückung bei Kontakt

$p$

Strömungsmechanischer Druck

$\Delta p \quad$ Druckunterschied

Aufweitdruck beim hydraulischen Aufweiten

$Q \quad$ Querkraft

Linienlast auf ein Rohr

$q_{i}$

Zeitabhängiger Anteil der Biegelinie im Separationsansatz

$R \quad$ Leitblechbohrungsradius

$r \quad$ Radiuskoordinate

Re Reynoldszahl

$r_{I}$

Radius der inneren Oberfläche eines Sichelspaltes

$S$

Leitblechdicke

Rohrplattendicke

Belastungsfaktor in halbempirischen Stabilitätskriterien

$S_{C}$

Quellterm in numerischen Gleichungen

Sr Strouhal-Zahl

$s_{i j}$

Deformationstensor

$T_{i j}$

Testfilter-Spannungstensor

$t_{i j}$

Physikalische Zeit

$\Delta T$

Feinstruktur-Spannungstensor

Meßzeit

$u$

Zeitschrittweite im numerischen Modell

$u_{0}$

Strömungsgeschwindigkeit in $x$-Richtung

$u_{i}$

Zuströmgeschwindigkeit im Stutzen oder im Kanal

$\Delta u$

$i$-te kartesische Komponente der Strömungsgeschwindigkeit

Zusatzterm in Konvektionsschemata höherer Ordnung

$u_{s p}$

Spaltgeschwindigkeit 


$\begin{array}{ll}u_{s p, k r i t} & \text { Kritische Spaltgeschwindigkeit } \\ v & \text { Strömungsgeschwindigkeit in } y \text {-Richtung } \\ v_{R} & \text { Betrag des Geschwindigkeitsvektors eines Rohres in der Bohrung } \\ w & \text { Biegelinie im Balkenmodell der Rohre } \\ w & \text { Strömungsgeschwindigkeit in } z \text {-Richtung } \\ \Delta w & \text { Radiale Verschiebung eines Rohres im Leitblech } \\ w_{i} & \text { Ortsabhängiger Anteil der Biegelinie im Separationsansatz } \\ w_{n} & \text { Verschiebung in Stoßrichtung bei Kontakt } \\ w_{r} & \text { Radiale Strömungsgeschwindigkeit im Sichelspalt } \\ w_{\varphi} & \text { Azimutale Strömungsgeschwindigkeit im Sichelspalt } \\ w_{x} & \text { Axiale Strömungsgeschwindigkeit im Sichelspalt } \\ x & \text { 1. kartesische Koordinate } \\ \Delta x & \text { Zellkantenlänge in } x \text {-Richtung } \\ x_{i} & \text { i-te kartesische Koordinate } \\ y & \text { 2. kartesische Koordinate } \\ \Delta y & \text { Zellkantenlänge in } y \text {-Richtung } \\ z & \text { 3. kartesische Koordinate } \\ \Delta z & \text { Zellkantenlänge in } z \text {-Richtung }\end{array}$

\section{Griechische Symbole}

$\alpha \quad$ Empirischer Stabilitätsexponent im Connors-Kriterium

$\alpha \quad$ Stabilitätsparameter im Newmark-Schema

$\alpha \quad$ Hertzscher Exponent im Kontaktmodell

$\alpha_{i} \quad$ Exponent des Axiallasteinflusses auf die Rohreigenfrequenz

$\beta \quad$ Winkel der Geschwindigkeitsrichtung eines Rohres im Leitblech

$\beta_{i} \quad$ Eigenwert für analytische Rohreigenfrequenzberechnung

$\hat{\beta}_{i} \quad i$-ter Eigenwert der Biegeschwingungen bei Axiallast

$\beta_{V} \quad$ Verkippwinkel zwischen Rohr und Leitblech

$\gamma \quad$ Winkel der Richtung der Verschiebung eines Rohres im Leitblech

$\Delta \quad$ Kantenlänge eines finiten Strömungsvolumens

$\Delta \quad$ Dämpfungsparameter im Connors-Kriterium

$\overline{\bar{\Delta}} \quad$ Testfiltergröße

$\delta \quad$ Dämpfungsparameter im Newmark-Schema

$\delta_{i j} \quad$ Kronecker-Symbol 
$\varepsilon \quad$ Turbulente Dissipation je Masseneinheit

$\varepsilon \quad$ Oberflächendeformation im Reibungsmodell

$\varepsilon_{0} \quad$ Grenzoberflächendeformation im Reibungsmodell

$\dot{\varepsilon}_{t} \quad$ Grenzgeschwindigkeit im Reibungsmodell

$\Phi \quad$ normierte Biegeschwingungseigenform

$\varphi \quad$ Umfangswinkel

$\hat{\varphi} \quad$ Axiallastverhältnis

$\kappa \quad$ Ersatzhülsenparameter nach Kohlpaintner

$\eta \quad$ Stoff- bzw. Gesamtviskosität des Fluids im Mantelraum

$\eta_{T} \quad$ Turbulente Viskosität

$\Lambda \quad$ Logarithmisches Dekrement

$\lambda \quad$ Lagerfaktor im Strukturmodell

$\lambda^{*} \quad$ Skalare Stoffgröße

$\lambda_{i} \quad$ Eigenwerte der Leckagefaktoren

$\mu_{0} \quad$ Deformationsunabhängiger Haftreibungskoeffizient

$\mu_{G l} \quad$ Geschwindigkeitsunabhängiger Gleitreibungskoeffizient

$\mu_{t} \quad$ Geschwindigkeitsabhängiger Gleitreibungskoeffizient

$\rho \quad$ Fluiddichte der Mantelraumströmung

$\rho_{R} \quad$ Dichte des Rohrwerkstoffs

$\sigma_{F} \quad$ Fließgrenze des Werkstoffs

Querteilungsverhältnis der Berohrung T/D

$\tau_{i j} \quad$ Spannungstensor

$\omega \quad$ Spezifische turbulente Dissipationsrate

$\hat{\omega}_{i} \quad i$-ter Eigenwert der Biegeschwingungen bei Axiallast

normierte Rohrlängsachsenkoordinate

$\Psi$

normierte Verteilung der Rohrqueranströmgeschwindigkeit

$\psi_{i} \quad i$-ter Eigenwert der Biegeknickdifferentialgleichung

Strukturdämpfung bzw. Fluid-Struktur-Dämpfung

$\zeta_{n}$

Numerische Zusatzdämpfung bei Kontakt in Stoßrichtung

$\zeta_{t}$

Numerische Zusatzdämpfung bei Kontakt in Tangentialrichtung 


\section{Kopfzeiger}

* Konvektierende Strömungsgeschwindigkeit

1, 2, 3 Zeiger für Bereiche der Biegelinie

$D \quad$ Druck

$n \quad$ Nummer des Zeitschritts

$R \quad$ Reibung

$T \quad$ Transponierung eines Vektors oder einer Matrix

\section{Fußzeiger}

Azimutalkoordinate

I Numerischer Zähler in $x$-Richtung

$i \quad$ Indizierung kartesischer Tensoren oder Zählvariable

$J \quad$ Numerischer Zähler in $y$-Richtung

$j \quad$ Indizierung kartesischer Tensoren

$K \quad$ Numerischer Zähler in $z$-Richtung

$L \quad$ Knoten links von einem Leitblechlager

$n b \quad$ Nachbarwerte

$R \quad$ Knoten rechts von einem Leitblechlager

$R \quad$ Rohr

$r \quad$ Radiuskoordinate

RMS root mean square - Quadratischer Mittelwert

$s \quad$ Knoten in einem Leitblech

sp $\quad$ Spalt

$T, B, E, \quad$ Indizierung von Zellzentren nach Kompaßnotation $W, N, S, P$

$t, b, e, w, \quad$ Indizierung von Zellseitenflächen nach Kompaßnotation

$n, s$

$x, y, z \quad$ Kartesische Koordinatenrichtungen

\section{Operatoren}

Tiefpaßfilterung auf dem Simulationsgitter

Tiefpaßfilterung auf dem Testgitter

Mittelwertbildung

Schwankungswert bzw. Korrekturwert 


\section{Einleitung}

\subsection{Funktionsprinzip, wirtschaftliche Aspekte und Schadensfälle}

Rohrbündelwärmetauscher sind häufig anzutreffende Komponenten z.B. im Apparate- und Anlagenbau, der Chemieingenieurtechnik, der Verfahrenstechnik, der pharmazeutischen Industrie, sowie in konventionellen Kraftwerken und kerntechnischen Anlagen [Kle00]. Sie werden verwendet, um in einem gewünschten Prozeß z.B. ein heißes Fluid zu kühlen oder ein kaltes Fluid zu erwärmen. Eine typische Bauform ist in Abbildung 1-1 dargestellt. Ein Rohrbündel wird von zwei Rohrplatten gehalten, z.B. ist die eine Rohrplatte fest mit dem Behälter verbunden und die andere etwa als Schwimmkopf ausgeführt. Die kreiszylindrischen Rohre des Bündels können fluchtend oder versetzt angeordnet sein. Durch das Innere der Rohre strömt z.B. das heiße Fluid des Primärkreislaufs, während die Rohre an der Außenseite im Gegenstrom vom Kühlmedium des Sekundärkreislaufs umströmt werden. Leitbleche als Einbauten sorgen für eine mäanderförmige Durchströmung des Mantelraums. Dabei kann es sich bei den Fluiden um eine Flüssigkeit, ein Gas oder ein mehrphasiges Fluid handeln.

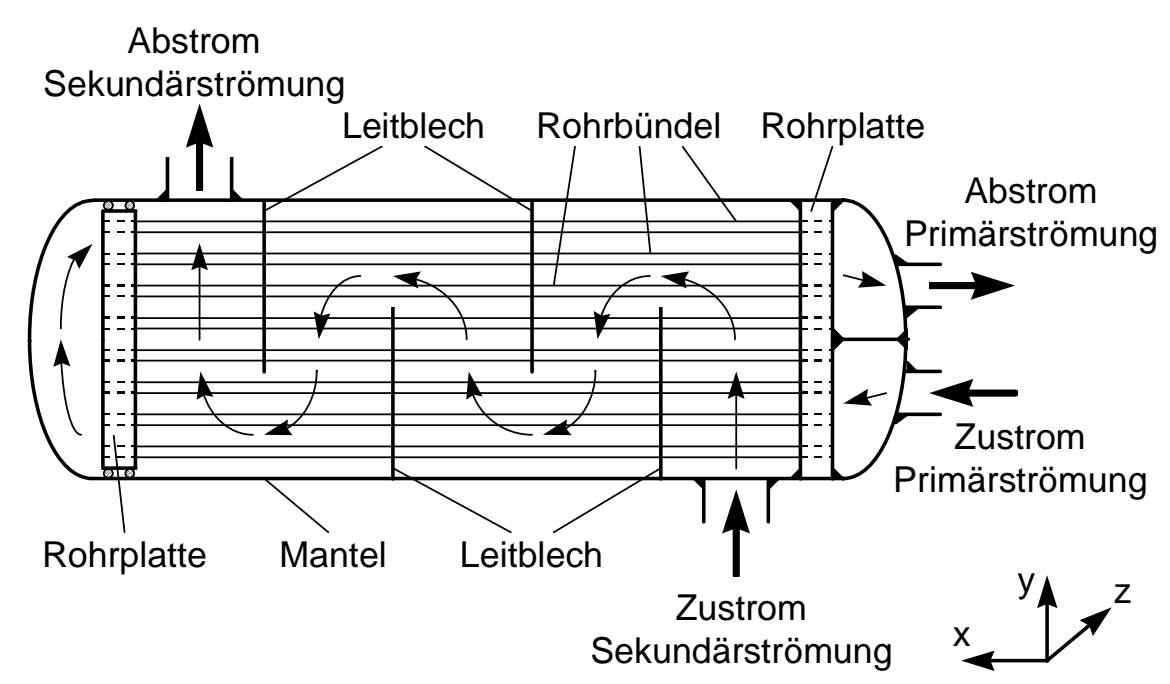

Abbildung 1-1: Funktionsprinzip des Geradrohrbündelwärmetauschers mit Koordinatensystem für die vorliegende Arbeit.

Unter dem betriebswirtschaftlichen Zwang [Ker84], einen immer höheren thermischen Wirkungsgrad erzielen zu müssen, war man zunehmend dazu überge- 
gangen, die Durchströmungsgeschwindigkeiten zu erhöhen und die Rohrwandstärken immer weiter zu reduzieren. Dies führte jedoch zu einer Reihe von Schadensfällen [Bau85, Kot84, Tro86] infolge der durch die Außenströmung induzierten Schwingungen der Rohrbündelstruktur. Ein Überblick über solche Schadensfälle wird auch in [Pai80] gegeben. Ein weiteres Problem tritt auf, wenn nach einiger Betriebszeit aus produktionswirtschaftlichen Gründen zu entscheiden ist, ob die Produktionsrate und damit die Anströmgeschwindigkeit des Rohrbündelapparates noch gesteigert werden kann, ohne Schwingungsschäden zu riskieren.

Die Schädigungen können in der Folge zum Stillstand der betroffenen Anlage oder zu Unfällen führen. Der betroffene Betrieb erleidet bei Produktionsstillstand einen hohen finanziellen Schaden infolge des Produktionsausfalls, ggf. noch durch zusätzliche Entschädigungszahlungen an Auftraggeber. Die Ausfallzeiten sind im Schadensfall für gewöhnlich deshalb lang, weil im Anlagenbau jede Apparatekomponente als Einzelstuick beschafft wird. Ein Ersatzapparat steht daher in aller Regel nicht sofort zur Verfügung. Außerdem läuft der produzierende Betrieb bei Anlagenstillstand Gefahr, den Auftraggeber als Kunden überhaupt zu verlieren. Insbesondere dann, wenn es sich um ein Unternehmen mit geringer Kapitaldecke handelt, können die finanziellen Schäden infolge eines Schwingungsschadens an einem Rohrbündelapparat in die Millionen gehen [Fis02] und zur Insolvenz des Unternehmens führen. Betriebsschließungen sind dann mit Arbeitsplatzverlusten verbunden.

Bei Unfällen können weitere Komponenten der Anlage zerstört werden. Die Reparatur ist dadurch erschwert oder verzögert und führt zu weiteren finanziellen Nachteilen. Auch Personenschäden sind bereits aufgetreten. Versicherungsunternehmen haben im Jahr 1998 an Anlagen, die der Störfallverordnung unterliegen, 2 Tote, 168 Verletzte und Schäden in Millionenhöhe gemeldet, wobei mechanisches Versagen einen hohen Prozentsatz der Schadensursachen darstellt [Fis00a]. Wegen des existenziellen wirtschaftlichen Risikos und des Sicherheitsrisikos haben sowohl der Lieferant als auch der Verwender eines Rohrbündelapparates ein natürliches Interesse daran, die beschriebenen Schwingungschäden zu verhindern.

\subsection{Erregermechanismen}

Als Reaktion auf die genannten Schadensfälle begann man etwa Ende der Sechziger Jahre mit intensiven Forschungsarbeiten auf dem Gebiet der strömungsin- 
duzierten Schwingungen in Rohrbündelwärmetauschern. So unterscheidet man inzwischen verschiedene strömungsmechanische Anregungsmechanismen [Ku189]. Bei Anregung der Struktur durch wechselseitige Wirbelablösung kann die Wirbelablösefrequenz der Struktureigenfrequenz zu nahe kommen, es tritt Resonanz ein (Lock-In-Effekt), und die Schwingungsamplituden wachsen beliebig an. Beim Buffeting wird die Struktur durch Turbulenz in der Strömung angeregt. Dabei filtert die Struktur gewissermaßen den Anteil aus dem Turbulenzspektrum heraus, der ihrer Eigenfrequenz entspricht. Durch die gegenseitige Beeinflussung der Strömungsfelder um die einzelnen Rohre kann es zu einer fluidelastischen Kopplung kommen. Dabei wirkt das Fluid zwischen den Rohren des Bündels wie eine elastische Feder, wodurch das Bündel insgesamt ab einer gewissen Spaltgeschwindigkeit zu Flatterschwingungen angeregt wird. Weitere Anregungsmechanismen sind in [Kul89] beschrieben.

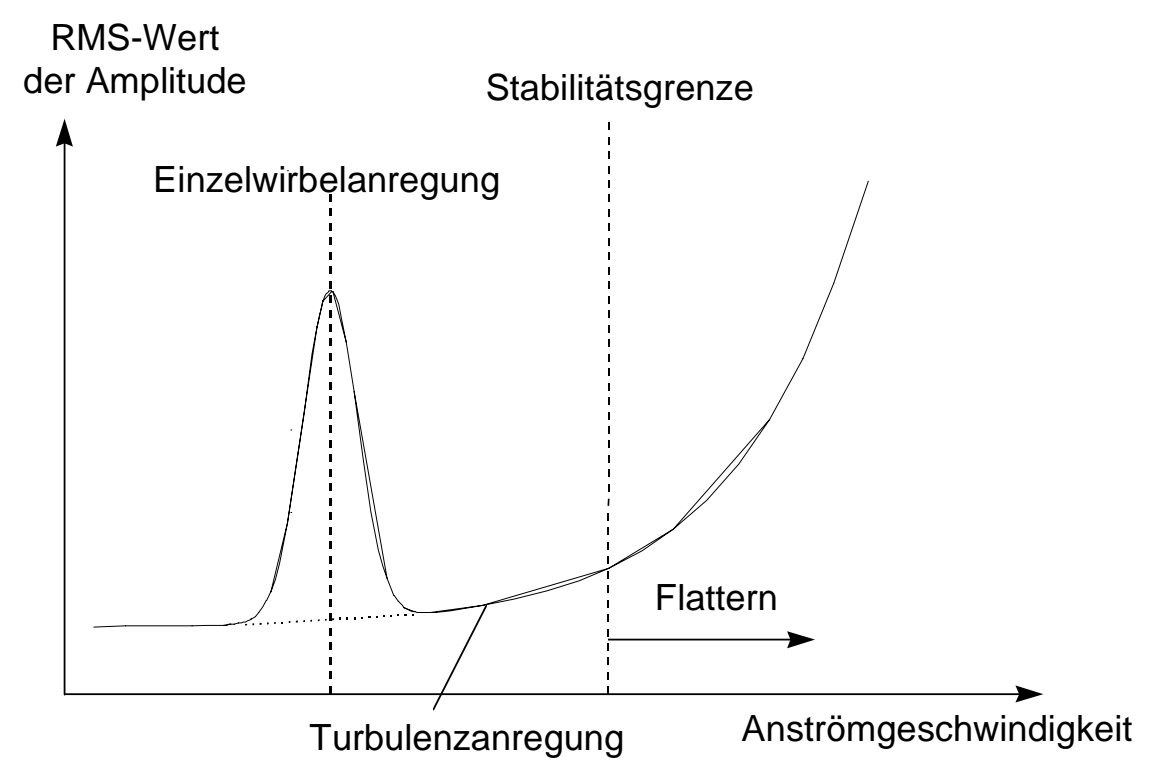

Abbildung 1-2: Prinzipwirkung von strömungsmechanischen Anregungsmechanismen auf die Schwingungsamplituden in Rohrbündeln.

Die Schwingungen der Rohrstruktur infolge der strömungsmechanischen Anregung bleiben entweder bei geringer und mäßiger Amplitude stabil oder werden aufgrund von Resonanz- oder Flattererscheinungen mit über alle Grenzen wachsenden Amplituden instabil. Abbildung 1-2 zeigt die Prinzipwirkung der jeweiligen Anregungsmechanismen auf die Amplituden der Rohrschwingungen. Das Erreichen des instabilen Punktes hängt dabei entscheidend von der Zuströmge- 
schwindigkeit ab. Die Zuströmgeschwindigkeit bei einsetzender fluidelastischer Instabilität wird „kritisch“ genannt. Instabil schwingende Rohre werden nach kürzester Zeit zerstört, während stabil schwingende Rohre einem länger andauernden Verschleißprozeß ausgesetzt sind. Insbesondere findet an den als Stützstellen wirkenden Leitblechen ein verstärkter Abrieb des Rohrmaterials statt.

\subsection{Bisherige Absicherung gegen Rohrbündelschwingungen}

Für die bisherige halbempirische Absicherung gegen strömungsinduzierte Schwingungen in Rohrbündelwärmetauschern müssen die einzelnen bekannten Anregungsmechanismen getrennt voneinander abgeprüft werden. Eine Reihe von halbempirischen Stabilitätskriterien werden z.B. in [Pai80, Pet91, Pri95, VDI97] zusammengetragen. Die Wichtigsten von ihnen werden nachfolgend kurz dargestellt.

Die Absicherung gegen Einzelwirbelablösung an Einzelrohren bzw. in Rohrbündeln ausreichend großer Teilung wird mit Hilfe der Strouhalzahl vorgenommen. Die Strouhalzahlen für Wirbelablösungen erster bis dritter Ordnung sind für einen weiten Reynoldszahlenbereich durch eine große Anzahl von Experimenten, die in der Literatur dokumentiert sind, genau genug bekannt [VDI97]. Aus den Strouhalzahlen werden die Wirbelablösefrequenzen berechnet. Mit Hilfe der damit bekannten Frequenz der strömungsmechanischen Erregerkräfte kann im stabilen Fall die Beanspruchung eines Rohrs über die Betrachtung der entsprechenden Bewegungsgleichungen bestimmt werden. Als Faustregel gilt darüber hinaus, daß die Wirbelablösefrequenz höchstens $70 \%$ der Eigenfrequenz der Einzelrohre erreichen darf, um den Lock-In, das Überspringen der Wirbelablösefrequenz auf die Rohreigenfrequenz, und damit einen resonanten Schwingungsfall mit theoretisch unendlich großen Amplituden, zu verhindern [VDI97]. Einzelrohrresonanzen können über das beschriebene Vorgehen gut abgesichert werden.

Komplizierter gestalten sich die Verhältnisse bei fluidelastischen Instabilitäten. Die experimentell gewonnenen Erkenntnisse und Ergebnisse über den Zusammenhang zwischen den statistisch gemittelten Rohramplituden und der kritischen Anströmgeschwindigkeit wurden in Ermangelung besserer Methoden in halbempirischen Stabilitätskriterien zusammengefaßt. Bekanntestes Beispiel ist das erweiterte Connors-Kriterium [Con78, VDI97] 


$$
\frac{u_{s p, k r i t}}{f_{1} D}=\frac{K}{\sqrt{S}}\left(\frac{m \Lambda}{D^{2} \rho}\right)^{\alpha} \text { mit } S=\frac{\int_{0}^{1} \Psi^{2}(\xi) \cdot \Phi^{2}(\xi) d \xi}{\int_{0}^{1} \Phi^{2}(\xi) d \xi}
$$

mit empirischen Konstanten $K$ und $\alpha$, sowie einem Belastungsfaktor $S$, der den Einfluß der normierten inhomogenen Geschwindigkeitsverteilung $\Psi$ und des normierten Schwingungsmodes $\Phi$ auf das Stabilitätsverhalten erfassen soll. Die normierte Geschwindigkeitsverteilung wird entweder halbempirisch als vereinfachtes Kastenprofil bestimmt [VDI97] oder aus stationären CFDBerechnungen gewonnen [Ley93, Ge194, Urb94, Jah95, Ge198]. Bei großen Wärmetauschern können die Bündel durch poröse Strömungszellen modelliert [Bot00, Pri98] werden. Eine Reihe von Autoren geben modifizierte Kriterien an [Pai80, Pet91, Pri95]. Die vorhandenen halbempirischen Stabilitätskriterien gegen Rohrbündelschwingungen sind oft auf reale Behältergeometrien schlecht anwendbar, da sie dem Konstrukteur schwer zugängliche Werte enthalten (z.B. logarithmisches Dekrement $\Lambda$, dominierende Eigenfrequenz, meist $f_{1}$ angenommen, empirische Faktoren $K$ und $\alpha$, Spaltgeschwindigkeiten $u_{s p}$ ) und dadurch Unsicherheiten in sich bergen. Das Kriterium von Troidl [Tro86, Kas94]

$$
\begin{array}{ccc} 
& \tau<1,68 & \tau>1,68 \\
\text { 30 -Anordnung: } & \frac{u_{s p, k r i t}}{f_{1} D}=\frac{1,09 \cdot(\tau+1)}{\sqrt{S}} & \frac{u_{s p, k r i t}}{f_{1} D}=\frac{3,5}{\sqrt{S}} \\
\text { 90 -Anordnung: } & \frac{u_{s p, k r i t}}{f_{1} D}=\frac{2,08 \tau}{\sqrt{S}} & \frac{u_{s p, k r i t}}{f_{1} D}=\frac{3,5}{\sqrt{S}}
\end{array}
$$

vermeidet zur Stabilitätsbeurteilung immerhin die Verwendung des am schwierigsten zu bestimmenden logarithmischen Dekrements $\Lambda$. Es ist aber nicht auf andere Medien als Wasser übertragbar. Grundlegende Schwäche aller halbempirischen Stabilitätskriterien ist darüberhinaus, daß nur Aussagen über die unterste Stabilitätsgrenze getroffen werden können, aber prinzipiell keine Aussagen über die Dauerschwingbelastung der Rohre im zeitfesten Bereich sowie über die Lebensdauer möglich sind. Um die Sicherheit der Anlage trotzdem zu gewährleisten, werden die Kriterien so verwendet, daß in der Praxis die kritische Anströmgeschwindigkeit des Bündels um bis zu $500 \%$ zu hoch vorhergesagt wird [Kas97a], d.h., daß die Anlage nur einen Bruchteil ihres Potentials ausnutzt. 
Bei Turbulenzerregung steigen die Amplituden nahezu linear mit der Anströmgeschwindigkeit an, so daß erhöhte Amplituden über Ähnlichkeitsbetrachtungen reduziert werden können. Schäden infolge von Turbulenzerregung bestehen meist in erhöhtem Abrieb an den Lagerstellen im Leitblech [AuY98, Ble79, Ble85, Cha87, Che85, KoP84]. Zur Absicherung ist jedoch die Kenntnis eines Turbulenzspektrums in der betrachteten Konfiguration notwendig [VDI97]. Dieses ist dem auslegenden Konstrukteur in den meisten Fällen aber nicht bekannt.

Die Absicherung gegen akustische Resonanzen erfolgt analytisch über Betrachtungen zu stehenden Wellen in Luftsäulen quer zur Anströmrichtung und quer zur Rohrachse [VDI97]. In der vorliegenden Arbeit werden allerdings nur inkompressible Medien berücksichtigt, so daß akustische Resonanzen nicht auftreten können.

\subsection{Literaturübersicht: Numerische Arbeiten}

Nachdem die numerische Strömungsmechanik, z.B. in der Automobilbranche, der Nukleartechnik und der Luft- und Raumfahrtindustrie, schon seit vielen Jahren als Berechnungswerkzeug eingesetzt wird, beginnen mit der zunehmenden Entwicklung kommerzieller strömungsmechanischer Programme [AEA97] auch die konventionelle Kraftwerkstechnik [Bir00] und die Verfahrenstechnik [Bra98], CFD als Werkzeug einzusetzen. Bischoff [Bis93] berechnet z.B. den Wärmeübergang in Rohrbündeln auf der Basis des CFD-Codes PHOENICS. Kaam [Kaa00] und Kretschmar [Kre00] generieren mit dem CFD-Code CFX [AEA97] Modelle von Rohrbündelwärmetauschern mit starren Rohren unter Berücksichtigung der Leitblechspalte und berechnen die Kräfte auf die Rohre in den einzelnen Strömungssektionen. Sawtschenko [Saw97] berechnet mit Hilfe von PHOENICS und FIDAP die Strömungsverhältnisse der Primärströmung in der Haube eines Rohrbündelwärmetauschers. Hamid [Ham97] berechnet die Strömungs- und Temperaturverteilungen in großen Rohrbündelwärmetauschern mit Hilfe von CFX [AEA97]. Leitbleche werden dabei als Porositäten dargestellt. In [Ley93, Gel94, Urb94, Jah95, Ge198] werden Strömungsverteilungen in großen Wärmetauschern mit Hilfe von STAR-CD berechnet, um den Stabilitätsfaktor des Connors-Kriteriums zu bestimmen. In [Bir00] wird hierfür CFX verwendet.

In der Forschung wird dagegen im Bereich der Rohrbündelschwingungen wegen der Unzulänglichkeiten halbempirischer Stabilitätskriterien schon seit etwa 
20 Jahren darauf hingearbeitet, Rohrbündelschwingungen numerisch zu simulieren. Die aus den Strömungskräften resultierende Fluiddämpfung wird implizit mitgerechnet und muß nicht wie etwa beim Connors-Kriterium [Con78, Kas94, Kas97a] vom Konstrukteur aus Ausschwingversuchen ermittelt werden. Außerdem sind mit der Vorgehensweise der Simulation Aussagen über die Höhe der Schwingungsamplituden möglich.

In [Kas97a] ist eine umfangreiche Literaturübersicht zu den bis 1995 entstandenen Arbeiten auf dem Gebiet der numerischen Simulation von Einzelrohrumströmungen bzw. der Rohrreihen- und Rohrbündeldurchströmungen enthalten. Als numerische Methoden kamen die Finite-Differenzen-Methode, die FiniteElemente-Methode und die Finite-Volumen-Methode zum Einsatz. Dabei ist zunächst zu unterscheiden zwischen stationärer und instationärer Berechnung. Stationäre Berechnungen [Gor78, Cha89] sind im Zusammenhang mit dem Ziel der Simulation von Rohrbündelschwingungen eher als historisch durch die zunächst zur Verfügung stehende mangelhafte Rechnerleistung bedingte erste numerische Gehversuche zu werten. Der für die vorliegende Arbeit notwendige zeitabhängige Strömungskraftverlauf ist so grundsätzlich nicht zu ermitteln.

Weiter ist zu unterscheiden zwischen Rechnungen mit starren Rohren oder elastisch gelagerten Rohren. Instationäre zweidimensionale Rechnungen an starren Einzelrohren werden in [Kaw83, Kaw84, Bra85, Son90, Fra91] durchgeführt. In [Sin89] wird eine starre Rohrreihe analysiert. In [Has90] werden die Turbulenzspektren der Kräfte auf ein zweidimensional durchströmtes starres Rohrbündel mit Hilfe eines modifizierten LES-Turbulenzmodells sowie teilblockierter Zellen berechnet. Eine weitere Rechnung für zweidimensionale starre Rohrbündel tritt 1994 auf [Sat94].

Für höhere Reynoldszahlen ist die Einführung von Turbulenzmodellen notwendig. Hierfür liegen bis 1997 nur Arbeiten für starre Rohre bzw. Bündel in zwei und drei Dimensionen vor [Son90, Fra91, Bar94]. Hier werden aber auch schon Reynoldszahlen bis 8400000 [Son90] erreicht. Als Turbulenzmodelle wurden Large-Eddy-Modelle, Standard- $k$ - $\varepsilon$-Modelle und LRR-Modelle verwendet. In [Bar94] wird dabei ein in [Bar83] entwickeltes modifiziertes LES-Modell verwendet.

Elastisch gelagerte Rohre bzw. Bündel in zweidimensionaler Rechnung werden in [Tam88, Ich94, Ich95, Sad95] verwendet. Hierbei werden aber keine Turbulenzmodelle berücksichtigt, so daß der Einsatz der Verfahren auch bei Nutzung 
der leistungsfähigsten Computer auf den Reynoldszahlenbereich bis ca. 18000 beschränkt bleibt.

Auch neueste Arbeiten befassen sich oft nur mit der zweidimensionalen Umströmung von Einzelzylindern bei Reynoldszahlen bis maximal 5000 [Cha00, Liu00, Gui00]. In [Fuj01] werden mit einem nicht näher benannten CFD-Code 2D-Strömungen um Einzelzylinder bis zu Reynoldszahlen von 1000000 berechnet. Allerdings stimmen die ab $R e=10000$ berechneten Strouhalzahlen nicht mehr mit Literaturwerten überein. Die Bewegung des steifen Zylinders wird mit Hilfe eines Feder-Masse-Schwingers in der schon von Kassera [Kas97a] verwendeten Art berechnet.

In [Wil00] wird eine Quasi-3D-Rechnung vorgestellt, bei der einzelne Strömungsebenen um einen Zylinder mit einem 2D-Stromfunktionsverfahren berechnet werden. Allerdings werden nur Reynoldszahl bis 100 berücksichtigt. In [Nak01] wird CFX zur Berechnung des 3D-Strömungsfeldes um einen Einzelzylinder verwendet. Die Bewegung des steifen Zylinders wird ebenso wie bei [Fuj01] mit Hilfe eines Feder-Masse-Schwingers berechnet. Die Reynoldszahl ist auf 1000 beschränkt. Der Widerstandsbeiwert ist mit 1,29 etwas zu hoch, während die Strouhalzahl mit 0,21 korrekt berechnet wird. Der Auftriebsbeiwert wird nicht angegeben. Um die Ablösung hervorrufen zu können, werden 120 Zellen am Zylinderumfang benötigt.

Kassera [Kas97a] entwickelte bis 1997 das Programmpaket FIVSIC-2D für inkompressible zweidimensionale Fälle, das mit der Kontinuitätsgleichung und der Navier-Stokes-Gleichung die Grundgleichungen der Strömungsmechanik simulierte und die Kräfte auf die Rohre, die als Feder-Masse-Schwinger modelliert waren, über Summation der Oberflächendrücke darstellte. Hierfür verwendet er die Finite-Volumen-Methode nach Patankar [Pat80] und erstmals ein $k$ - $\omega$ Turbulenzmodell [Wi194]. Mit dem QUICK-Verfahren [Leo79] wurde auch ein konvektives Schema höherer als erster Ordnung implementiert. Dadurch konnten nach einer Reihe in der Literatur dokumentierter Arbeiten über Teilaspekte der zweidimensionalen Simulation fluid-struktur-gekoppelter Einzelrohr- bzw. Rohrbündelschwingungen erstmals fluid-struktur-gekoppelte Schwingungssimulationen für ganze Rohrbündel unter Beachtung der Turbulenz bis zu Reynoldszahlen von 200000 durchgeführt werden.

Schröder [Schr99] führte instationäre zweidimensionale Rechnungen für Rohrbündel mit dem kommerziellen CFD-Paket STAR-CD durch und koppelte die CFD-Rechnungen mit einem nicht näher bezeichneten FE-Programm für die 
Berechnung der dynamischen Rohrantwort. Schröder testet mehrere Turbulenzmodelle $(k-\varepsilon$, Zwei-Zonen- $k-\varepsilon$ und $k-\omega)$ und kommt zu dem Ergebnis, daß das von Kassera [Kas97a] benutzte $k$ - $\omega$-Modell [Wi194] die besten Resultate liefert. Das von Kassera benutzte Stufengitter [Kas97a] liefert mit STAR-CD oszillierende Geschwindigkeitsverläufe im Nachlauf der Zylinder. Ebenso wie Kassera verwendet Schröder das QUICK-Diskretisierungsverfahren [Leo79]. Schröder erhält relativ gute Übereinstimmungen mit experimentellen Ergebnissen. Reale Wärmetauschergeometrien mit Leitblechspalten und Kontakt zwischen Rohren und Leitblechen einerseits oder von Rohren untereinander andererseits sind aber in seinen Simulationen nicht enthalten. Schröder verwendet im Gegensatz zu Kassera ein körperangepaßtes unstrukturiertes Gitter. Damit ist sein Verfahren im Gegensatz zu dem von Kassera [Kas97a] und der vorliegenden Arbeit nicht in der Lage, sich sehr nahe kommende oder sich berührende Rohre zu berücksichtigen, da das Gitter hierdurch zu stark deformiert wird und regeneriert werden müßte.

Zum Stand der Literatur kann zum Zeitpunkt des Abschlusses der vorliegenden Arbeit zusammenfassend festgestellt werden, daß es noch keine Veröffentlichung gibt, in der ein realistisches Rohrbündelmodell unter Berücksichtigung von Turbulenz, dreidimensionaler Strömung im Mantelraum und Kontakt zwischen Rohren und Leitblechen auf Basis eines numerischen Simulationscodes hinsichtlich strömungsinduzierter Schwingungen behandelt wird. Dies ist zu diesem Zeitpunkt auch die vorherrschende Meinung in Übersichtsbeiträgen der internationalen Literatur [Wea00, Pri01].

\subsection{Ziel der vorliegenden Arbeit}

Die vorhandenen halbempirischen Stabilitätskriterien gegen Rohrbündelschwingungen sind meist schlecht anwendbar, da sie dem Konstrukteur schwer zugängliche Werte enthalten, auf reale Apparate schwierig zu übertragen sind und dadurch Unsicherheiten in sich bergen. Grundlegende Schwäche der Stabilitätskriterien ist darüberhinaus, daß nur Aussagen über den Abstand der Anströmgeschwindigkeit von einer Stabilitätsgrenze getroffen werden können, jedoch keine Aussagen über die Dauerschwingbelastung der Rohre im zeitfesten Bereich möglich sind. Schädigungen aufgrund von Dauerbeanspruchung können damit nicht erklärt werden. Frühzeitige Schädigungen können sich insbesondere dann ergeben, wenn das Rohrbündel auf der ansteigenden Flanke der Verstärkerfunktion zu einem instabilen Bereich hin betrieben wird. Eine kleine 
Veränderung der Anströmgeschwindigkeit kann dann eine große Auswirkung auf die Schwingungsamplitude und damit auf die Lebensdauer haben.

Auch an zweidimensional rechnenden Simulationsverfahren [Kas97a, Schr99] ergeben sich noch erhebliche Kritikpunkte. Die mit Spalt versehenen Lagerungen im Leitblech können nicht berücksichtigt werden, so daß innerhalb des Strukturmodells das Lagerungsverhalten der Rohre nicht richtig wiedergegeben wird. Der in der Praxis wichtige Fall der Schädigung der Rohre infolge des dauernden Abriebs der Rohre an den Lagerstellen in den Leitblechen wird einer Betrachtung grundsätzlich entzogen. Es können keine realen Geometrien berechnet werden, der Einfluß der Anzahl und Positionen der Leitbleche und evtl. vorhandener weiterer Einbauten auf die Strömungsführung ist somit nicht in der Rechnung enthalten. Durch die Darstellung der Rohre im zweidimensionalen Modell als Feder-Masse-Schwinger kann nur die erste Eigenfrequenz der Rohre erfaßt werden. Aus den Experimenten zur vorliegenden Arbeit wurde jedoch deutlich, daß z.B. bei Rohren, die bei einem Drittel ihrer Länge gelagert sind, strömungsabhängig die dritte und sechste Eigenfrequenz dominant werden können.

Im Rahmen der vorliegenden Arbeit sollte daher als Fortsetzung der Arbeiten von Kassera [Kas97a] der dreidimensional rechnende Code FIVSIC-3D entwikkelt werden, der auch die mit Spalt versehenen Lagerungen im Leitblech, die dreidimensionale Strömung, die Rohre als elastisches Kontinuum sowie die dreidimensionale Geometrie von Wärmetauschern berücksichtigt, so daß den Hauptkritikpunkten an den halbempirischen Auslegungskriterien und den zweidimensionalen Simulationen prinzipiell abgeholfen ist:

- Innerhalb des Strukturmodells wird das Lagerungsverhalten unter Berücksichtigung von teils aktiven, teils inaktiven Lagerstellen in den Leitblechen richtig wiedergegeben,

- dadurch wird der Verschleiß der Rohre an den Lagerstellen in den Leitblechen einer Betrachtung zugänglich,

- Leckageströmungen an den Leitblechen sind in den Betrachtungen enthalten,

- die aus den Strömungskräften resultierende Fluiddämpfung und die hydrodynamischen Zusatzmassen werden implizit mitgerechnet,

- es können reale Geometrien und damit reale Strömungsführungen berechnet werden,

- es sind Aussagen über die Höhe der Amplituden möglich und 
- damit ist die Lebensdauer prinzipiell bei Kenntnis einer Wöhlerlinie über die Schadensakkumulationshypothese von Palmgren und Miner nach [Rom98] berechenbar.

Kommerzielle CFD-Programme sind zum gegenwärtigen Zeitpunkt nicht in der Lage, voll fluid-struktur-gekoppelte Probleme bei praxisrelevanten Reynoldszahlen zu berechnen [Wea00]. Für die vorliegende Arbeit mußten gängige Algorithmen, wie sie z.T. auch in kommerziellen CFD-Programmen für die Strömungsberechnung eingesetzt werden, für den eigens entwickelten Simulationscode stark verändert und angepaßt werden, um stabile und konvergente Ergebnisse zu erhalten. Dies wäre bei ausschließlicher Verwendung kommerzieller Programme nicht möglich gewesen, da der hierfür notwendige tiefe Eingriff in den Programmtext aus urheberschutzrechtlichen Gründen nicht zugelassen wird. Außerdem soll eine Abhängigkeit von vorhandenen kommerziellen Programmen vermieden werden. Eine Versionsänderung bei einem kommerziellen Code hat oft den Fortfall aller Wartungsmöglichkeiten durch den Vertreiber zur Folge. Daher wurde der in dieser Arbeit entwickelte Algorithmus in Fortran 90 implementiert.

Dreidimensionale strömungsmechanische Rechnungen sind wegen der fortschreitenden Computertechnik seit kurzem überhaupt erst möglich. Es besteht inzwischen kein Zweifel mehr daran, daß nur numerische Simulationsverfahren das Potential besitzen, die Rohrbündelstruktur so zu optimieren, daß bei erhöhter Wirtschaftlichkeit und Sicherheit der thermische Wirkungsgrad verbessert wird [Wea00]. Die Industrie sieht wegen der hohen potentiellen Schadenssummen ebenfalls eine Notwendigkeit zur Entwicklung verbesserter Auslegungsund Nachrechnungsverfahren zu den beschriebenen Schwingungsproblemen. Eine größere Anzahl in- und ausländischer Unternehmen des Apparate- und Anlagenbaus hat bereits ihr Interesse an dem im Rahmen der vorliegenden Arbeit entwickelten Verfahren bekundet [Fis99c].

\subsection{Gliederung der Arbeit}

Im folgenden Kapitel 2 wird das im Rahmen der vorliegenden Arbeit entwikkelte numerische Berechnungsverfahren vorgestellt. Dabei werden nach jedem wichtigen Entwicklungsabschnitt die Teilmodule des Algorithmus mit Hilfe von Testrechnungen, Testmessungen oder Literaturrecherchen auf ihre Funktionsfähigkeit überprüft. Die drei Hauptentwicklungsschritte sind dabei das Strömungsmodul, das Strukturmodell, sowie die Kopplung der beiden Module. In 
Kapitel 3 wird der Aufbau des Versuchsstands beschrieben, mit dessen Hilfe Meßergebnisse der Rohrbewegungen in wirklichkeitsgetreuen Rohrbündelmodellen ermittelt werden konnten. Die Plausibilität der Meßergebnisse wird unabhängig vom numerischen Verfahren anhand analytischer Überlegungen belegt. Gleichzeitig werden anhand der eigenen Messungen Schwächen des Konzepts der hydraulischen Zusatzmassen und der bisherigen Auslegungsverfahren diskutiert. In Kapitel 4 erfolgt eine Gegenüberstellung der gemessenen und simulierten Ergebnisse für eine Beispielkonfiguration, mit der das Berechnungsverfahren überprüft wurde. Außerdem werden die Ergebnisse Resultaten gemäß der bisher verwendeten halbempirischen Auslegungsmethode gegenübergestellt, um die Unterschiede und den Fortschritt herauszuarbeiten. In Kapitel 5 erfolgt eine abschließende Zusammenfassung und Bewertung der Ergebnisse der vorliegenden Arbeit. In Kapitel 6 sind die für die Anfertigung der Arbeit verwendeten Literaturstellen aufgelistet. Aus drucktechnischen Gründen findet sich als Anhang in Kapitel 7 eine Zusammenstellung von Farbgrafiken. Hier sind insbesonders numerisch simulierte und mit Hilfe eines eigens hierfür entwickelten Postprocessors visualisierte Strömungsbilder zu sehen. 


\section{Das numerische Simulationsverfahren}

\subsection{Simulation des Strömungsfeldes im Mantelraum}

\subsubsection{Strömungsmechanische Grundgleichungen}

Zur Herleitung der inkompressiblen instationären Impulsgleichungen der Strömungsmechanik ist eine Vielzahl von Lehrbüchern (z.B. [Tru68]) und Fachaufsätzen bekannt, sie soll daher hier nicht noch ein weiteres Mal wiederholt werden. Zur Turbulenzmodellierung gibt es ebenfalls eine Unzahl von Literaturstellen (z.B. [Wi194, Fri94, Wen93, Ber96]), die hier ebenfalls nicht alle zitiert werden können. Daher wird für beide Problemkreise lediglich das kurz dargestellt, was hinsichtlich der Auswahl der numerischen Simulationstechnik im vorliegenden Verfahren von Bedeutung ist.

Die Navier-Stokes-Gleichungen stellen letztlich die Maxwellschen Transportgleichungen für den Impuls der Strömung dar und können entweder aus der Sicht der Makromechanik aus dem dynamischen Kräftegleichgewicht an einem infinitesimalen Fluidelement oder aus der Sicht der Mikromechanik durch Bildung der Momente der Boltzmann-Gleichung hergeleitet werden [Fri94]. Für ein dichteveränderliches Fluid erhält man in differentieller tensorieller Schreibweise [Tru68, Kli89, Fri94]

$$
\frac{\partial\left(\rho u_{i}\right)}{\partial t}+\frac{\partial\left(\rho u_{i} u_{j}\right)}{\partial x_{j}}=-\frac{\partial p}{\partial x_{i}}+\frac{\partial \tau_{i j}}{\partial x_{j}}
$$

Grundsätzlich sind die so dargestellten Impulsgleichungen geeignet, auch turbulente Strömungen in Direkter Numerischer Simulation (DNS) zu berechnen. Es kann jedoch gezeigt werden, daß die Anzahl der zur räumlichen Auflösung der Strömung notwendigen Gitterpunkte im turbulenten Fall mit $R e^{9 / 4}$ und die Anzahl der notwendigen Zeitschritte zur zeitlichen Auflösung der turbulenten Strömungsvorgänge mit $R e^{3 / 4}$ ansteigt [Wen93]. Mithin ergeben sich für praktische Ingenieuranwendungen große Speicheranforderungen, die mit heutigen Rechnern nicht zu erfüllen sind. Zudem sorgt das später noch darzustellende CFL-Kriterium dafür, daß die Ergebnisse aus numerischer Simulation (CFD) für Ingenieuranwendungen auch deshalb nicht innerhalb einer vernünftigen Rechenzeit zu erwarten sind, weil mit höherer Netzauflösung auch aus Stabilitätsgründen kleinere Zeitschritte zu verwenden sind. Für kleine Reynoldszahlen 
werden oft DNS-Ergebnisse als Vergleichsrechnung verwendet. Für größere Reynoldszahlen muß man sich aber mit sogenannten Turbulenzmodellen behelfen. Für den allgemeinen Fall der turbulenten Strömung wird für alle Strömungsgrößen eine Aufspaltung in einen Mittelwert und einen Schwankungswert vorgenommen. Man setzt [Fri94, Wi194]

$$
\begin{gathered}
p=\langle p\rangle+p^{\prime} \\
u_{i}=\left\langle u_{i}\right\rangle+u_{i}^{\prime} \\
\tau_{i j}=\left\langle\tau_{i j}\right\rangle+\tau_{i j}^{\prime}
\end{gathered}
$$

ein und mittelt die Strömungsgleichungen. Dadurch erhält man unter Berücksichtigung der Kontinuitätsgleichung [Tru68, Fri94, Wi194]

$$
\frac{\partial\left\langle u_{i}\right\rangle}{\partial x_{i}}=0
$$

für den inkompressiblen Fall die gemittelte Impulsgleichung [Wi194, Wen93]

$$
\rho \frac{\partial\left\langle u_{i}\right\rangle}{\partial t}+\rho\left\langle u_{j}\right\rangle \frac{\partial\left\langle u_{i}\right\rangle}{\partial x_{j}}=-\frac{\partial\langle p\rangle}{\partial x_{i}}+\frac{\partial\left\langle\tau_{i j}\right\rangle}{\partial x_{j}}-\rho \frac{\partial\left\langle u_{i}^{\prime} u_{j}^{\prime}\right\rangle}{\partial x_{j}}
$$

Aus den konvektiven Anteilen der Impulsgleichung resultiert bei der Mittelungsoperation ein Tensor, der die gemittelten Korrelationen der Schwankungen der Geschwindigkeiten enthält. Dieser Tensor wird als Reynoldsscher Schubspannungstensor bezeichnet. Aus der Sicht der Makromechanik bewirkt er eine zusätzliche Reibungskraftwirkung am Fluidelement infolge der turbulenten Schwankungsbewegungen der Strömung, aus der Sicht der Mikromechanik beschreibt er den mittleren turbulenten Impulsaustausch zwischen den Strömungsrichtungen. Der Tensor ist symmetrisch und enthält somit sechs weitere unabhängige Unbekannte des Gleichungssystems, für die zunächst keine weiteren Gleichungen vorliegen. Dies nennt man das Schließungsproblem der Turbulenztheorie [Fri94, Wen93].

Es gibt eine Reihe von Ansätzen zur Modellierung der sechs zusätzlich auftretenden Schubspannungskomponenten [Wi194, Wen93]. Nach der Anzahl der neu eingeführten Differentialgleichungen werden sie als Null-, Ein-, Zwei- oder Mehrgleichungsmodelle bezeichnet. Ein mögliches Mehrgleichungsmodell ist das sogenannte Reynoldssche Schubspannungsmodell, bei dem die sechs neuen Unbekannten über sechs weitere Schubspannungstransportgleichungen be- 
schrieben werden. Die zu lösenden Differentialgleichungen enthalten aber neue unbekannte Korrelationen, die wiederum modelliert werden müssen. Insofern verschiebt dieses Modell das Schließungsproblem nur auf eine höhere Ebene. Die Anzahl von Unbekannten kann aber auf nur noch eine Unbekannte reduziert werden. Für den mittleren Spannungstensor wird die Annahme

$$
\left\langle\tau_{i j}\right\rangle=2 \eta\left\langle s_{i j}\right\rangle+\delta_{i j} \lambda^{*}\left\langle s_{k k}\right\rangle
$$

getroffen [Fri94], die als Newtonsche Schubspannungshypothese bezeichnet wird und auch für laminare Strömungen gültig ist. Dabei wird

$$
\left\langle s_{i j}\right\rangle=\frac{1}{2}\left(\frac{\partial\left\langle u_{i}\right\rangle}{\partial x_{j}}+\frac{\partial\left\langle u_{j}\right\rangle}{\partial x_{i}}\right)
$$

als Deformationstensor bezeichnet. Für den Reynoldsschen Schubspannungstensor wird die Annahme von Boussinesq [Wil94]

$$
-\rho\left\langle u_{i}^{\prime} u_{j}^{\prime}\right\rangle=2 \eta_{T}\left\langle s_{i j}\right\rangle-\frac{2}{3} \rho\left\langle s_{k k}\right\rangle \delta_{i j}
$$

getroffen. Beide Spannungshypothesen werden eingesetzt, und da wegen der Massenerhaltung (2-3) der zweite Term auf der rechten Seite von (2-7) entfällt, erhält man für den vorliegenden inkompressiblen Fall die Gleichung

$$
\left.\rho \frac{\partial\left\langle u_{i}\right\rangle}{\partial t}+\rho\left\langle u_{j}\right\rangle \frac{\partial\left\langle u_{i}\right\rangle}{\partial x_{j}}=-\frac{\partial\langle p\rangle}{\partial x_{i}}+\frac{\partial}{\partial x_{j}}\left(\eta+\eta_{T}\right)\left(\frac{\partial\left\langle u_{i}\right\rangle}{\partial x_{j}}+\frac{\partial\left\langle u_{j}\right\rangle}{\partial x_{i}}\right)\right)
$$

Die Turbulenz der Strömung wird also über einen zusätzlichen Beitrag zur Viskosität in die Impulsgleichungen eingebracht. Sie stellt für das strömungsmechanische Gleichungssystem nur noch eine weitere unbekannte Größe dar.

Bekannte Zwei-Gleichungs-Modelle zur Berechnung der turbulenten Viskosität sind das $k$ - $\varepsilon$-Modell und das $k$ - $\omega$-Modell [Wi194], bei denen je eine zusätzliche Transportgleichung für die Energie $k$ der turbulenten Schwankungsbewegungen und für die turbulente Dissipation $\omega$ bzw. $\varepsilon$ zu lösen sind, aus denen dann die zusätzliche Turbulenzviskosität berechnet wird. Beide Modelle enthalten jedoch Modellkonstanten. Somit liefern diese Modelle für ein Strömungsproblem evtl. sogar sehr gute Ergebnisse, für ein anderes jedoch nicht. Ihre Allgemeingültigkeit ist also nicht gegeben. Insbesondere das $k$ - $\varepsilon$-Modell hat sich wegen der 
Voraussetzung der isotropen Turbulenz [Wil94] für viele komplexere Strömungsanwendungen, auch für Zylinderumströmung [Fra91] bzw. Rohrbündeldurchströmung, als nicht geeignet herausgestellt. Mit dem $k$ - $\omega$-Modell konnte Kassera bei der zweidimensionalen Simulation schwingender Rohrbündel hingegen gute Ergebnisse erreichen [Kas97a,b]. Außerdem ist vorteilhaft, daß ohne Verwendung von Randfunktionen durch die Grenzschicht hindurchintegriert werden darf [Wi194, Kas97a]. Jedoch müssen zwei weitere Differentialgleichungen gelöst werden, von denen sich die $\omega$-Gleichung steif verhält, was die Konvergenzrate des Gesamtverfahrens verringert.

Aufgrund der kurz dargestellten Problematik wurde die sogenannte LargeEddy-Simulationstechnik (LES) in den letzten Jahren stark weiterentwickelt [Wi194, Ber96]. Dabei wird das großskalige Strömungsfeld ähnlich dem Vorgehen in der DNS-Technik direkt berechnet, während Wirbelstrukturen unterhalb einer Filtergröße häufig durch zusätzliche Viskosität modelliert werden. Die Mittelung wird räumlich durchgeführt und ist im Sinne der LES-Theorie als Tiefpaßfilterung zu verstehen. Formal ergeben sich dieselben Gleichungen wie in (2-2) bis (2-8), wenn die Mittelungsoperatorzeichen (Brackets) durch die Filterungsoperatorzeichen (einfache Überstreichung) ersetzt werden. Daher werden diese Gleichungen hier nicht noch einmal notiert. Nach dem Ansatz von Smagorinsky [Sma63, Wi194] wird in der vorliegenden Arbeit die zusätzliche Turbulenz nach der Gleichung

$$
\eta_{T}=\rho C(\Delta x \Delta y \Delta z)^{2 / 3} \sqrt{2 \bar{s}_{i j} \bar{s}_{i j}}
$$

berechnet. Dabei ist $C$ eine weitere unbekannte Konstante, die SmagorinskyKonstante, die extern vorgegeben sein muß. Sie liegt beim StandardSmagorinsky-Modell [Sma63, Wi194] typischerweise im Bereich von 0,01 bis 0,0576. In den Arbeiten zum ursprünglichen einfachen Smagorinsky-Modell wurde statt $C$ ein Term $C_{S}{ }^{2}$ verwendet, so daß sich typische Werte für $C_{S}$ von 0,1 bis 0,24 ergeben. Kassera hat für die zweidimensionale Simulation schwingender Rohrbündel mit dem dargestellten LES-Modell gute Ergebnisse erzielen können [Kas95]. Problematisch war jedoch, daß die Qualität der Ergebnisse stark von der Wahl der Konstanten abhängig war. Um diesen Rechenaufwand für Variationsrechnungen zu vermeiden, ging Kassera zum $k$ - $\omega$-Modell über [Kas97a].

Bernert und Eppler [Ber96] untersuchen in ihrem Bericht zum Stand der LESTechnik eine sehr große Anzahl von Literaturstellen und LES-Modellen. Große 
Unterstützung finden dabei sogenannte dynamische Modelle, bei denen die Konstante $C$ als räumlich und zeitlich variabel betrachtet wird. Als Berechnungsgrundlage dienen dabei lokale Betrachtungen der verschiedenen Turbulenzgrößen. Bernert und Eppler empfehlen aufgrund ihrer umfangreichen Studien das sogenannte Feinstruktur-Modell von Lilly [Li192] und Germano [Ger91], das im Folgenden dargestellt werden soll. Im nachfolgenden Textteil ist die Überstreichung einer Größe als die Darstellung einer auf dem Simulationsfilter räumlich tiefpaßgefilterten Größe zu verstehen. Die strömungsmechanischen Größen werden analog zu (2-2) als Summe aus einer tiefpaßgefilterten Größe und einer Schwankungsgröße dargestellt. Der Feinstruktur-Spannungstensor $t_{i j}$ ist definiert als

$$
t_{i j}=\bar{u}_{i} \bar{u}_{j}-\overline{u_{i} u_{j}}
$$

Wird auf die räumlich gefilterten Navier-Stokes-Gleichungen ein zweites gröberes Filter (Testfilter) angewendet, so entsteht auf der rechten Seite der Testfilter-Spannungstensor $T_{i j}$

$$
T_{i j}=\overline{\bar{u}}_{i} \overline{\bar{u}}_{j}-\overline{\overline{\overline{u_{i} u_{j}}}}
$$

Die Filterung auf dem gröberen Gitter ist durch doppelte Überstreichung angedeutet. Die Filterungen werden in der vorliegenden Arbeit numerisch mittels eines Rechteckfilters nach Trapezregel [Ber96] durchgeführt. Die beiden Turbulenzprobleme werden geschlossen über die Modellgleichungen

$$
\begin{aligned}
& t_{i j}-\frac{1}{3} \delta_{i j} t_{k k}=2 C \Delta^{2}|\bar{s}| \bar{s}_{i j} \\
& T_{i j}-\frac{1}{3} \delta_{i j} T_{k k}=2 C \overline{\bar{\Delta}}^{2}|\overline{\bar{s}}| \overline{\bar{S}}_{i j}
\end{aligned}
$$

wobei die Testfiltergröße mit $\overline{\bar{\Delta}}=2 \Delta$ bezeichnet ist. Die beiden Modellgleichungen können nur konsistent sein, wenn die Konstante $C$ lokal passend gewählt wird [Ger91]. Hierzu wird die Differenz zwischen dem Spannungstensor $T_{i j}$ auf dem gröberen Gitter und dem mit der gröberen Maschenweite gefilterten Spannungstensor des feineren Gitters gebildet. Dabei entsteht der test-windowSpannungstensor

$$
L_{i j}=T_{i j}-\overline{\bar{t}}_{i j}=-\overline{\overline{\bar{u}_{i} \bar{u}_{j}}}+\overline{\bar{u}}_{i} \overline{\bar{u}}_{j}
$$


Die test-window-Spannungen können direkt ausgewertet und mit dem SchlieBungsansatz verglichen werden. Dazu wird die Gleichung

$$
L_{i j}-\frac{1}{3} \delta_{i j} L_{k k}=2 C \overline{\bar{\Delta}}^{2}|\overline{\bar{S}}| \overline{\bar{S}}_{i j}-2 \overline{\overline{C \Delta^{2}|\bar{S}| \bar{S}_{i j}}}
$$

gebildet. (2-14) stellt ein System von fünf unabhängigen Intergralgleichungen für nur eine Funktion $C$ dar, da $C$ im Filterungsoperator erscheint. Lilly [Li192] umgeht das Problem, indem er die Funktion $C$ vor den Filterungsoperator zieht, was an sich unzulässig ist. Zur Anpassung der nur noch einen freien Konstante $C$ schlägt er einen Ansatz nach der minimalen Fehlerquadratmethode vor. Mit der Definition

$$
M_{i j}=\overline{\bar{\Delta}}^{2}|\overline{\bar{S}}| \overline{\bar{S}}_{i j}-\Delta^{2} \mid \overline{\overline{\bar{S} \mid \bar{S}_{i j}}}
$$

kann als Fehlergröße

$$
G=\left(L_{i j}-\frac{1}{3} \delta_{i j} L_{k k}-2 C M_{i j}\right)^{2}
$$

definiert werden. Der Fehler wird über die Bedingung $\partial G / \partial C=0$ minimiert. Der optimale Wert für $C$ ergibt sich somit unter Beachtung der Inkompressibilität (2-3) zu

$$
C=\frac{1}{2} \frac{L_{i j} M_{i j}}{M_{i j} M_{i j}}
$$

Daß es sich um ein Fehler-Minimum handeln muß, zeigt die zweite Ableitung von $G$ nach $C$, da $\partial^{2} G / \partial C^{2}=8 M_{i j} M_{i j}>0$ ist. Die Verwendung des dargestellten Smagorinsky-Turbulenzmodells in Verbindung mit der lokal optimierten Modellkonstante $C$ hat eine Reihe von Vorteilen gegenüber dem StandardLES-Modell oder bisherigen Zwei-Gleichungs-Modellen:

- Es müssen keine weiteren Transportgleichungen gelöst werden [Kas95].

- Es müssen keine Modellkonstanten [Kas95] verwendet werden, das Ergebnis des Modells ist insofern eindeutig.

- $C$ kann auch negativ werden, dadurch kann „,backscatter“ [Pio91], also der Energierücktransfer von kleinskaligen auf großskalige Wirbel, mitmodelliert werden. 
- Aus Sicht der Diskretisierung ergibt die vorgeschlagene Gittervergröberung um den Faktor Zwei [Ger91, Li192] den Vorteil, daß alle benötigten Größen unmittelbar vorliegen und nicht interpoliert werden müssen.

- Es darf ohne Verwendung von Randfunktionen durch die Grenzschicht hindurchintegriert werden [Mur99], es wird an Rändern also keine Dämpfungsfunktion, z.B. nach Van Driest, benötigt. Dies ist für die vorliegende Arbeit von Vorteil, da ja in einem Rohrbündelwärmetauscher eine große Anzahl von Oberflächen vorliegen.

- Die Qualität der Ergebnisse wird in der Literatur als sehr gut eingestuft [Ber96].

In der Literatur werden einige Stabilitätsprobleme mit dem dargestellten Modell beschrieben [Ber96, Mur99]. So verhält sich etwa das Modell sehr steif in dem Sinne, daß ein einmal negativ gewordener lokaler Wert für $C$ über eine lange Simulationsdauer negativ bleibt und somit die Stabilität des Verfahrens gefährdet. In der vorliegenden Arbeit wurde daher innerhalb eines Zeitschritts zwischen dem Standard-Modell und dem Lilly-Germano-Modell abhängig vom Residuum der Massenerhaltung hin- und hergesprungen. Wird das maximale Residuum größer, wird mit dem Standard-Modell und einem konstanten positiven Wert für $C$ gerechnet; dies wird v.a. bei Iterationen zu Beginn eines Zeitschritts der Fall sein. Durch die daraus entstehende Positivität der Koeffizienten des diskretisierten Lösungsschemas bleibt die Stabilität garantiert. Sinkt das Residuum von einem zum nächsten Iterationsschritt, wird das optimierende Modell verwendet; dies wird immer zum Ende des Zeitschritts hin der Fall sein. Daher liegen zum Ende des Zeitschritts auch immer die korrekt optimierten Werte für die turbulente Zähigkeit vor. Außerdem werden die in der Literatur beschriebenen typischen ,clipping“-Techniken zusätzlich angewandt:

- Die Gesamtviskosität muß immer größer als Null sein, damit die Koeffizienten des numerischen Systems immer positiv bleiben [Pat80]. Daher darf die turbulente Zusatzviskosität nicht kleiner als die negative Stoffviskosität werden.

- In [Ols94] wird die Verteilung der dynamischen Konstanten für das Beispiel einer Stufenerweiterung einer Rohrströmung berechnet. $38 \%$ bis $54 \%$ der Zellen enthalten demnach negative $C$-Werte, jedoch sind nur 2,2\% bis 3,3\% der Zellen mit Werten $C<-0,01$ belegt. Nur 0,029\% bis $0,64 \%$ der Zellen sind mit Werten $C>0,5$ belegt. Werte für $C<-0,01$ und Werte $C>0,5$ kön- 
nen daher [Ber96] folgend abgeschnitten werden, ohne daß die berechnete $C$-Verteilung zu stark verfälscht wird.

\subsubsection{Wahl eines numerischen Gitters}

Die strömungsmechanischen Erhaltungsgleichungen müssen auf einem numerischen Gitter diskretisiert werden, um punktweise gelöst werden zu können. Grundsätzlich gibt es die Möglichkeit strukturierter, teilstrukturierter und unstrukturierter Gitter. Unstrukturierte Gitter erfordern einen hohen programmiertechnischen Aufwand für die „Buchführung“ der einzelnen Zellen und Knoten [Irm94, Chu83]. Daher wurde diese Möglichkeit verworfen. Gitter können kartesisch oder körperangepaßt sein. Körperangepaßte Gitter haben zwar den Vorteil, in der Nähe eines umströmten Körpers sehr gut aufzulösen, allerdings ergibt sich eine aufwendige Herleitung, die den Programmcode anwachsen läßt [Fis95a]. Durch Kreuzterme und Coriolisterme in den Impuls- und Druckkorrekturgleichungen verlangsamt sich die Konvergenz, bei stark verzerrten Zellen kann das Verfahren instabil werden [Schö90]. Zudem hat sich rechnerisch in eigenen Untersuchungen gezeigt, daß für die Simulation der Wirbelablösung an zylindrischen Körpern bei körperangepaßten Koordinaten eine vergleichsweise sehr große Zellenanzahl am Umfang der Rohre notwendig ist. In [Nak01] wird dies bestätigt.
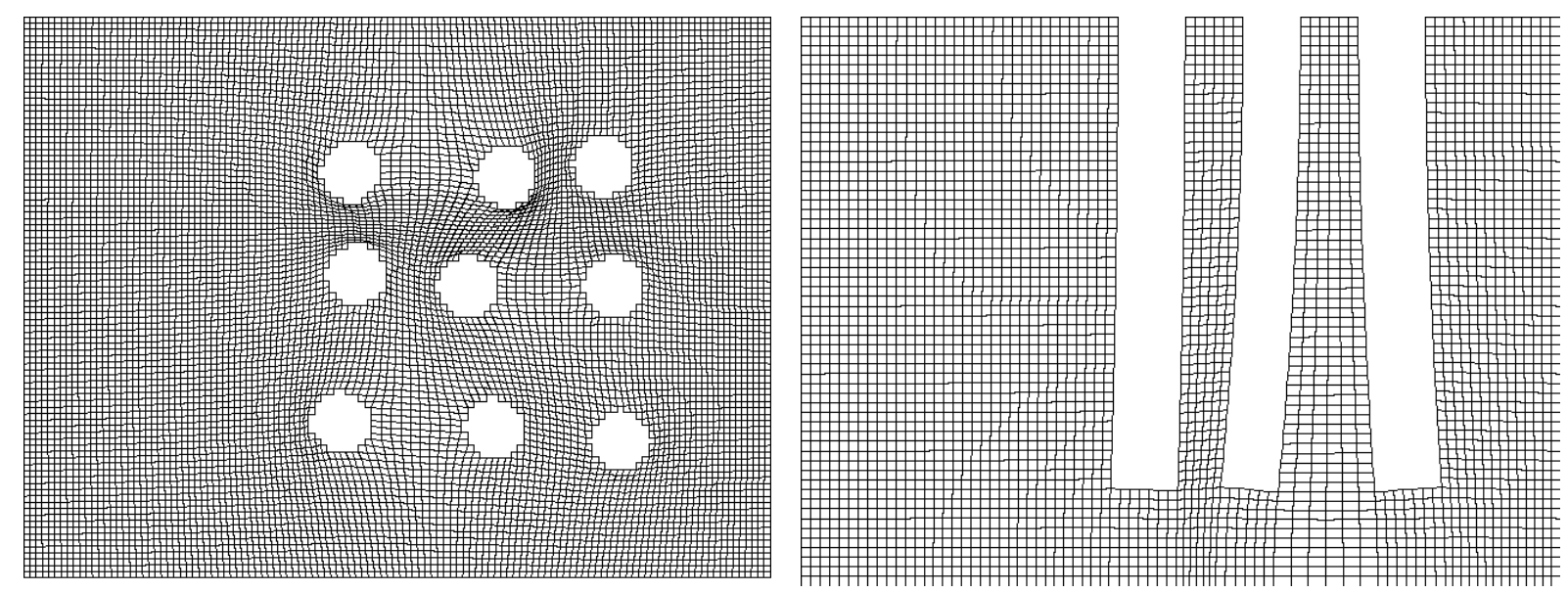

Abbildung 2-1: Orthogonale verzerrte 2D-Gitter mit ausblockierten

Zellen und oberflächennormalen Netzlinien.

Eine mögliche Lösung zur Umgehung starker Verzerrungen wären orthogonale Koordinaten [Ada84, Mob80, Hau81], jedoch kann gezeigt werden, daß diese im dreidimensionalen Raum allgemein nicht existieren [Hau81]. Daher wurde 
ein kartesisches Gitter gewählt, bei dem die Struktur durch Ausblockieren einzelner Zellen dargestellt wurde. Diese Vorgehensweise wurde zum Teil in [Kas97a] angewendet. In [Kas97a] werden jedoch teilstrukturierte Gitter verwendet, bei denen jedem Rohr ein feines Gitter zugeordnet wird, das entweder durch die Rohrbewegungen verzerrt oder über ein Grundgitter verschoben wird. Freitas [Fre97] verwendet in seinem 2D-Code ebenso wie Kassera [Kas97a] ein kartesisches Grundgitter und ein verschiebliches kartesisches Gitter, um die Umströmung eines schwingenden Vierkants zu berechnen. Die Kopplung zwischen den Gittern erfolgte in [Kas97a] und in [Fre97] durch Interpolationen. Kasseras Verfahren wurde dabei in manchen Fällen instabil [Kas98]. Abbildung 2-1 zeigt zwei der möglichen untersuchten Gitter, die nach der Methode von Eça [Eca96] berechnet wurden. Hier wurde der Versuch unternommen, wenigstens in bestimmten Berechnungsebenen orthogonale Gitter zu erzeugen. Probleme ergeben sich dann bei starker Rohrannäherung.

Endergebnis der Untersuchung verschiedener Gitterarten ist, daß aufgrund der Anforderungsliste (Speicherplatz sparen, Rechenzeit einsparen, extreme Fälle abdecken) körperangepaßte, teil- und unstrukturierte Gitter für die vorliegende Aufgabenstellung nicht in Frage kommen konnten. Schließlich wurde daher ein voll kartesisches Gitter gewählt, bei dem die Rohre durch teilweises Ausblokkieren von Zellen dargestellt werden. Die entwickelte Methode zur Darstellung der Verschieblichkeit der Rohre mit Hilfe eines Zellüberdeckungsgrades wird ausführlich in Abschnitt 2.3.1 (Seite 66) beschrieben.

\subsubsection{Diskretisierung der strömungsmechanischen Grundgleichungen}

$\mathrm{Zu}$ den Grundlagen des Gebiets der numerischen Strömungsmechanik (CFD) sind in letzter Zeit eine Reihe von umfangreichen Lehrbüchern (z.B. [Pat80, Schö90, No193, Gri95, Oer95, Fer99]) veröffentlicht worden. Deshalb soll hier nicht noch ein weiteres Mal eine umfassende Darstellung aller Grundlagen der CFD erläutert werden. Die verwendeten Gleichungen werden hier nur soweit dargestellt, wie es auf Basis einiger CFD-Kenntnisse zum Verständnis des in der vorliegenden Arbeit entwickelten Berechnung sverfahrens notwendig ist.

Um das hier verwendete numerische Verfahren - ein gemischtes FiniteDifferenzen/Finite-Volumen-Verfahren - zu verdeutlichen, wird beispielhaft die Herleitung der diskretisierten Impulsgleichung für die $x$-Richtung dargestellt. Dabei werden im folgenden alle Strömungsgrößen als nach dem Verständnis der LES-Turbulenztheorie räumlich gefilterte Größen angesehen. Zur 
Vereinfachung wird deshalb auch die Überstreichung zur Kennzeichnung gefilterter Größen fortgelassen. Ebenfalls zur Vereinfachung wird die Gesamtviskosität nur noch mit $\eta$ bezeichnet. Es ist dabei die Summe aus Stoffviskosität und turbulenter Viskosität gemeint. Mithin ist diese Summe auch eine lokal und zeitlich veränderliche Größe.

Als numerisches Gitter wird ein in $y$ - und $z$-Richtung äquidistant unterteiltes kartesisches Gitter mit der Maschenweite $\Delta y=\Delta z=\Delta$ verwendet. Die kartesischen Geschwindigkeitskomponenten werden dabei auf den Seitenflächen der Kontrollvolumina gespeichert, die Größen Druck und Zähigkeit hingegen in der Zellmitte. Man spricht von einem versetzten Gitter (,staggered grid“, [Har65]), wodurch unphysikalische Oszillationen im Druckverlauf verhindert werden [No193, Schö90]. In den $y$-z-Ebenen liegt der Rohrspiegel. Die $y$-z-Ebenen werden als Hauptströmungsebenen bezeichnet. Die $x$-Richtung wird ebenfalls äquidistant unterteilt, aber gegenüber der Unterteilung in $y$ und $z$ um einen Faktor $1 / \xi$ gestreckt. Diese Raumrichtung entspricht dabei der Richtung der Rohrlängsachsen. Die gleiche Unterteilung $\Delta x$ wird auch für die Diskretisierung der Rohrbewegungsgleichungen verwendet, um die Interpolation der Kräfte, Geschwindigkeiten und Verschiebungen zwischen Strömungsmechanik und Strukturmechanik zu vereinfachen. Der Zusammenhang der Netzweiten ist also durch die Gleichung

$$
\Delta=\Delta x \cdot \xi
$$

gegeben.

Zunächst wird die $x$-Impulsgleichung zur einfacheren Handhabbarkeit umgeschrieben in

$$
\begin{gathered}
\rho \frac{\partial u}{\partial t}+\frac{\partial}{\partial x}\left(\rho u^{2}-\eta \frac{\partial u}{\partial x}\right)+\frac{\partial}{\partial y}\left(\rho u v-\eta \frac{\partial u}{\partial y}\right)+\frac{\partial}{\partial z}\left(\rho u w-\eta \frac{\partial u}{\partial z}\right)= \\
=-\frac{\partial p}{\partial x}+\frac{\partial \eta}{\partial x} \frac{\partial u}{\partial x}+\frac{\partial \eta}{\partial y} \frac{\partial v}{\partial x}+\frac{\partial \eta}{\partial z} \frac{\partial w}{\partial x}
\end{gathered}
$$

Diese Gleichung wird durch Finite-Differenzen-Bildung an einem um eine halbe Maschenweite in $x$-Richtung verschobenen Kontrollvolumen diskretisiert. Dabei wird zur Bezeichnung die Kompaßnotation gewählt [Schö90]. Damit ergeben sich für die $x$-Richtung Top- und Bottom-Seite, für die $y$-Richtung Eastund West-Seite und für die $z$-Richtung North- und South-Seite des Kontrollvolumens. Dabei werden die Terme in den Positionen ihrer Speicherung mit 
Großbuchstaben $T, B, E, W, N, S$ bezeichnet, die Terme auf den Kontrollvolumen-Seitenflächen mit kleinen Buchstaben $t, b, e, w, n, s$. Mit $P$ wird der Mittelpunkt des betrachteten Kontrollvolumens bezeichnet. Zur Vereinfachung der Darstellung wird von jeder vorkommenden Art von Termen nur jeweils eine Diskretisierung besprochen, die weiteren Terme der gleichen Klasse ergeben sich analog. Abbildung 2-2 verdeutlicht die Notation.

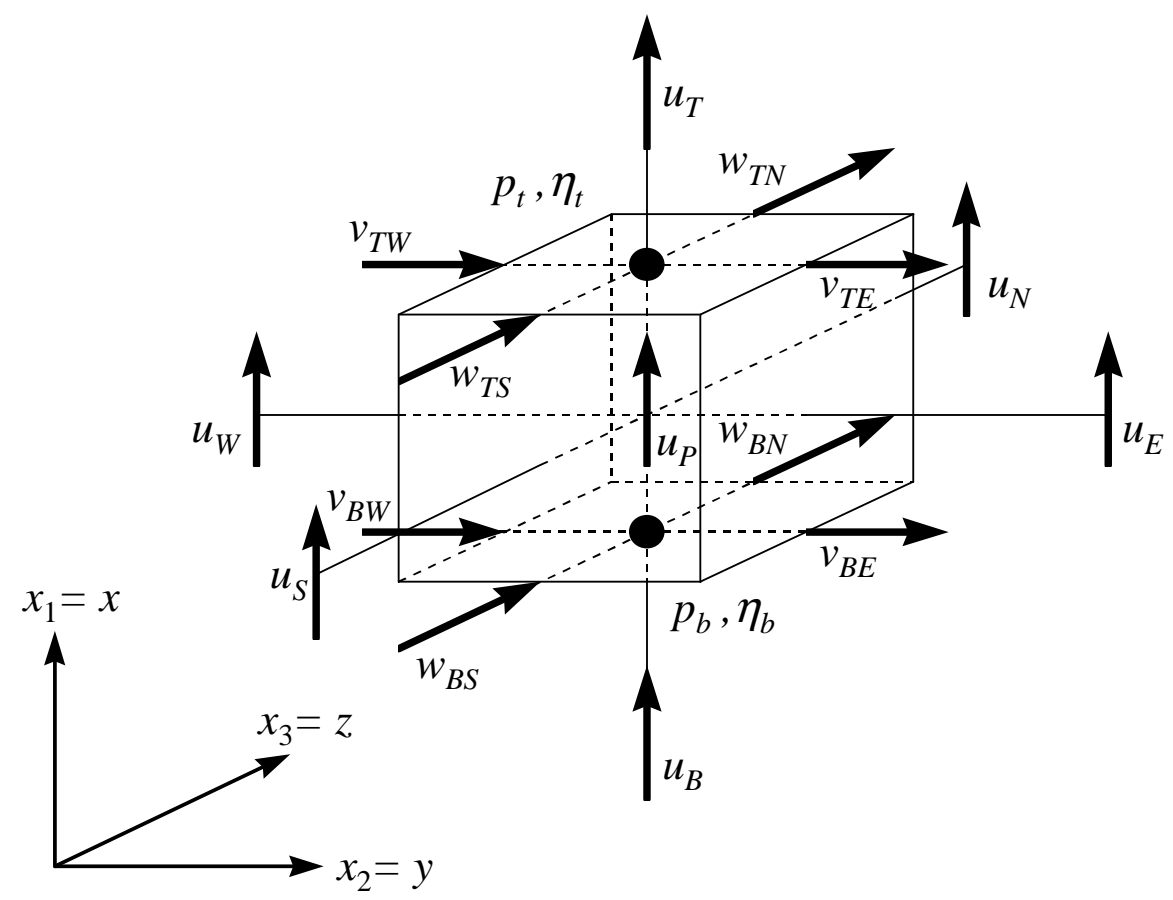

Abbildung 2-2: In $x$-Richtung um $\Delta x / 2$ versetztes Kontrollvolumen.

\subsubsection{Zeitliche Diskretisierung}

Für die zeitliche Diskretisierung wird eine Finite Differenz zweiter Ordnung gebildet, d.h., der gesamte Satz von Geschwindigkeiten muß zu drei Zeitebenen $n+1, n$ und $n-1$ vorgehalten werden. Durch Rückwärts-Diskretisierung ergibt sich für den Punkt $P$ der Finite-Differenzen-Quotient

$$
\left(\frac{\partial u}{\partial t}\right)_{P} \cong \frac{3 u_{P}-4 u_{P}^{n}+u_{P}^{n-1}}{2 \Delta t}
$$

Zur Vereinfachung der Schreibweise werden die oberen Indizes $n+1$ für alle Strömungsgrößen des neu zu berechnenden Zeitschritts fortgelassen. Da alle weiteren Diskretisierungen sich nur noch auf Größen des neuen Zeitschritts be- 
ziehen, erhält man ein vollständig implizites Verfahren. Hierdurch ergeben sich Vorteile hinsichtlich der Stabilität und des zu wählenden Zeitschritts im Vergleich zu expliziten Verfahren [Schö90].

\subsubsection{Druckgradient}

Aufgrund des versetzt angeordneten Gitters kann der Druckgradient ohne weitere Interpolationen als Finite Differenz von Knotenwerten dargestellt werden. Es ergibt sich z.B. für den Gradienten in $x$-Richtung

$$
\left(\frac{\partial p}{\partial x}\right)_{P} \cong \frac{p_{t}-p_{b}}{\Delta x}
$$

\subsubsection{Gemischte Konvektions/Diffusions-Terme}

Die räumlichen Ableitungen der gemischten Terme werden durch zentrale Finite-Differenzen-Bildung dargestellt. Für die Ableitung in $x$-Richtung ergibt sich z.B.

$$
\frac{\partial}{\partial x}\left(\rho u^{2}-\eta \frac{\partial u}{\partial x}\right)_{P} \cong \frac{\left(\rho u^{2}-\eta \frac{\partial u}{\partial x}\right)_{t}-\left(\rho u^{2}-\eta \frac{\partial u}{\partial x}\right)_{b}}{\Delta x}
$$

\subsubsection{Rein diffusive Terme}

Der noch innerhalb der Klammern verbleibende diffusive Term wird wiederum durch Finite Differenzen dargestellt. Es ergibt sich etwa

$$
\left(\eta \frac{\partial u}{\partial x}\right)_{t} \cong \eta_{t} \frac{u_{T}-u_{P}}{\Delta x}
$$

Auf der rechten Seite der umgeschriebenen $x$-Impulsgleichung (2-19) befindet sich noch ein rein dissipativer Term. Er resultiert aus der lokal veränderlichen Viskosität bei turbulenter Strömung und wird durch einfache FiniteDifferenzen-Bildung diskretisiert. Die dabei nicht direkt durch Knotenwerte darstellbaren Größen werden linear aus den daneben liegenden Knotenwerten interpoliert. Es ergibt sich etwa in der $x$-Impulsgleichung der Term

$$
\left(\frac{\partial \eta}{\partial x} \frac{\partial u}{\partial x}\right)_{P} \cong \frac{\eta_{t}-\eta_{b}}{\Delta x} \frac{u_{T}-u_{B}}{2 \Delta x}
$$




\subsubsection{Rein konvektive Terme}

Die rein konvektiven Terme bedürfen besonderer Aufmerksamkeit. Sie sind nichtlinear, da die gesuchten Größen, die Geschwindigkeiten, quadratisch vorkommen. Es wurde eine Reihe verschiedener Schemata für die Diskretisierung der konvektiven Terme entwickelt. Die in der vorliegenden Arbeit verwendeten Methoden sind UPWIND, PowerLaw, Hybrid-Verfahren und Monotonized Linear Upwind (MLU). Die diskretisierten Impulsgleichungen sollen aber zunächst anhand des UPWIND-Verfahrens [Pat80] dargestellt werden, die Implementierung der weiteren Verfahren ergibt sich dann durch Zusatzterme. Wichtige Forderungen an Differenzenschemata für konvektive Terme sind [Pat80]

- die Erhaltung der Transporteigenschaften der Impulsgleichungen,

- die Vermeidung unpysikalischer Überschwinger bei der Interpolation von Strömungsgrößen auf die Seitenflächen der Kontrollvolumina,

- Stabilität des resultierenden linearisierten Gleichungssystems,

- und die Genauigkeit der Diskretisierung.

Das UPWIND-Verfahren hat Transporteigenschaften, enthält keine Überschwinger, ist stabil, aber relativ ungenau. Der konvektive Term kann z.B. für die Top-Seitenfläche als

$$
u_{t} u_{t}=u_{t}^{*} u_{t}=\left\{\begin{array}{ll}
u_{t}^{*} u_{P} & \forall u_{t}^{*}>0 \\
u_{t}^{*} u_{T} & \forall u_{t}^{*}<0
\end{array} \quad u_{t}^{*}=\frac{u_{P}+u_{T}}{2}\right.
$$

dargestellt werden. UPWIND unterscheidet also zwischen konvektierender Größe $u_{t}{ }^{*}$ und konvektierter Größe $u_{t}$. Gesucht ist die Größe auf der Seitenfläche. Je nach Strömungsrichtung, also je nach Vorzeichen von $u_{t}{ }^{*}$, wird für die Größe auf der Seitenfläche die Größe des rechts oder links von der Seitenfläche liegenden Knotens, also $u_{P}$ oder $u_{T}$, gesetzt. Die UPWIND-Diskretisierung kann umgeschrieben werden in

$$
u_{t}^{*} u_{t}=u_{P}\left[\left[u_{t}^{*}, 0\right]\right]-u_{T}\left[\left[-u_{t}^{*}, 0\right]\right]
$$

mit dem Maximum-Operator

$$
[[A, B]]= \begin{cases}A & \forall A>B \\ B & \forall B>A\end{cases}
$$




\subsubsection{Zusammenfassung der Impulsgleichung}

Mit Hilfe der dargestellten Methoden wird nun die Impulsgleichung in $x$ Richtung diskretisiert. Die diskretisierte Kontinuitätsgleichung

$$
\frac{u_{t}^{*}-u_{b}^{*}}{\Delta x}+\frac{v_{e}^{*}-v_{w}^{*}}{\Delta}+\frac{w_{n}^{*}-w_{s}^{*}}{\Delta}=0
$$

wird mit $u_{P} \rho \Delta$ multipliziert und von der diskretisierten Impulsgleichung (2-19) abgezogen. Dadurch kann eine der Hauptforderungen bezüglich Stabilität von Patankar [Pat80] an ein numerisches Schema, nämlich die Hauptachsendominanz der entstehenden Systemgleichung, erfüllt werden. In der Form eines Gauss-Seidel-Point-By-Point-Solvers kann für $u_{P}$ die diskretisierte Gleichung

$$
u_{P}=\frac{a_{T} u_{T}+a_{B} u_{B}+a_{E} u_{E}+a_{W} u_{W}+a_{N} u_{N}+a_{S} u_{S}+4 a_{P}^{0} u_{P}^{n}-a_{P}^{0} u_{P}^{n-1}+S_{C}}{a_{T}+a_{B}+a_{E}+a_{W}+a_{N}+a_{S}+3 a_{P}^{0}}
$$

erhalten werden. In formelmäßiger Darstellung ergeben sich die Koeffizienten von (2-29) zu

$$
\begin{array}{ll}
a_{T}=\left[\left[-\xi \rho \Delta u_{t}^{*}, 0\right]\right]+\eta_{t} & u_{t}^{*}=\frac{u_{P}^{*}+u_{T}^{*}}{2} \\
a_{B}=\left[\left[\xi \rho \Delta u_{b}^{*}, 0\right]\right]+\eta_{b} & u_{b}^{*}=\frac{u_{B}^{*}+u_{P}^{*}}{2} \\
a_{E}=\left[\left[-\rho \Delta v_{e}^{*}, 0\right]\right]+\eta_{e} & v_{e}^{*}=\frac{v_{T E}^{*}+v_{B E}^{*}}{2} \\
a_{W}=\left[\left[\rho \Delta v_{w}^{*}, 0\right]\right]+\eta_{w} & v_{w}^{*}=\frac{v_{T W}^{*}+v_{B W}^{*}}{2} \\
a_{N}=\left[\left[-\rho \Delta w_{n}^{*}, 0\right]\right]+\eta_{n} & w_{n}^{*}=\frac{w_{T N}^{*}+w_{B N}^{*}}{2} \\
a_{S}=\left[\left[\rho \Delta w_{s}^{*}, 0\right]\right]+\eta_{s} & w_{s}^{*}=\frac{w_{T S}^{*}+w_{B S}^{*}}{2} \\
a_{P}^{0}=\frac{\rho \Delta^{2}}{2 \Delta t} & S_{C}=-\xi\left(p_{t}-p_{b}\right) \Delta+\frac{\partial \eta}{\partial x_{j}} \frac{\partial u_{j}}{\partial x} \Delta^{2}+ \\
+\rho \Delta\left\{-\xi(\Delta u)_{t} u_{t}^{*}+\xi(\Delta u)_{b} u_{b}^{*}-(\Delta u)_{e} v_{e}^{*}+(\Delta u)_{w} v_{w}^{*}-(\Delta u)_{n} w_{n}^{*}+(\Delta u)_{s} w_{s}^{*}\right\}
\end{array}
$$

Durch diese Art der Diskretisierung verbleiben immer nichtnegative Koeffizienten, dadurch bleibt nach Patankar die Stabilität gesichert. Nach Patankar [Pat80] sollte außerdem der Quellterm linearisiert werden, jedoch hat sich in der vorliegenden Arbeit die Konvergenzrate dadurch nicht nennenswert verbessert. Der diffusive Term aufgrund des Viskositätsgradienten wurde hier nicht voll 
ausgeschrieben, er wird, wie beschrieben, mit Finiten Differenzen dargestellt und enthält keinerlei Besonderheiten.

\subsubsection{Ergänzung um weitere konvektive Diskretisierungsschemata}

Das PowerLaw-Diskretisierungsschema stammt von Patankar [Pat80] und orientiert sich an der analytischen Lösung der eindimensionalen KonvektionsDiffusionsgleichung. Die dabei auftretende Exponentialfunktion wird durch eine Potenzfunktion fünfter Ordnung angenähert, um den Berechnungsaufwand geringer zu halten. Damit soll eine höhere Genauigkeit erreicht werden als beim einfachen UPWIND-Schema. Nach Patankar [Pat80] wird etwa der Koeffizient der positiven $x$-Kontrollvolumenseite durch

$$
a_{T}=\left[\left[-\xi \rho \Delta u_{t}^{*}, 0\right]\right]+\eta_{t}\left[\left[\left(1-0,1\left|-\xi \rho \Delta u_{t}^{*}\right| / \eta_{t}\right)^{5}, 0\right]\right]
$$

dargestellt. In der Praxis ergeben sich jedoch keine nennenswerten Verbesserungen der Genauigkeit. Der eindimensional analytisch lösbare Fall kann offenbar nicht ohne weiteres auf drei Dimensionen ausgeweitet werden.

Das Central Differencing Scheme (CDS) wurde zu Beginn der Programmentwicklung testweise verwendet, führte aber erwartungsgemäß aufgrund der wesentlich zu hohen Zell-Peclet-Zahlen zur Instabilität des Verfahrens bei praxisrelevanten Reynoldszahlen. Das Verfahren ist nur für Peclet-Zahlen kleiner als Zwei verwendbar [Pat80]. Das Hybrid-Verfahren schaltet deshalb je nach ZellPeclet-Zahl zwischen dem UPWIND-Verfahren erster Ordnung und dem Zentrale-Differenzen-Verfahren zweiter Ordnung um. Für den Koeffizienten der positiven $x$-Kontrollvolumenseite ergibt sich nach Patankar [Pat80] etwa

$$
a_{T}=\left[\left[-\xi \rho \Delta u_{t}^{*}, 0, \eta_{t}-\frac{1}{2} \xi \rho \Delta u_{t}^{*}\right]\right]
$$

Auch dieses Verfahren führte nicht zu nennenswerten Verbesserungen in der Genauigkeit.

Wegen der unzureichenden Genauigkeit der Verfahren erster Ordnung wurde auch mit hochauflösenden bzw. Verfahren höherer Ordnung gearbeitet. Aufgrund seiner Stabilitätseigenschaften bildet UPWIND die Grundlage vieler hochauflösender Schemata bzw. Diskretisierungsschemata höherer Ordnung. Solche Diskretisierungen können in Form eines Zusatzterms bei der Diskretisierung der konvektiven Flüsse eingebracht werden. Es ergibt sich dann z.B.

$$
u_{t}^{*} u_{t}=u_{P}\left[\left[u_{t}^{*}, 0\right]\right]-u_{T}\left[\left[-u_{t}^{*}, 0\right]\right]+(\Delta u)_{t} u_{t}^{*}
$$


Damit ergeben sich dieselben Koeffizienten wie beim UPWIND-Verfahren, die Zusatzterme höherer Ordnung können in den Quellterm eingebracht werden. Dadurch ergibt sich eine sehr effiziente Programmierung des Verfahrens bei alternativer Verwendung mehrerer Diskretisierungsschemata. Alle bisher vorgestellten Varianten sind letztlich Verfahren erster Ordnung. Die in der Herleitung der konvektiven Diskretisierungen beim UPWIND-Verfahren bereits notierten Zusatzterme $(\Delta u)_{t},(\Delta u)_{b}, \ldots$ sind für Verfahren erster Ordnung sämtlich gleich Null.

Eines der ersten entwickelten Verfahren höherer Ordnung, QUICK [Leo79], ist von dritter Ordnung genau, wurde aber nicht implementiert. Es führt zu unphysikalischen Überschwingern in den Lösungen, die das Verfahren divergieren lassen. Um zu stabilen Lösungen zu gelangen, muß es z.B. mit UPWIND gewichtet eingesetzt werden, wobei der Gewichtungsfaktor ein weiterer unbekannter Parameter ist, der die Genauigkeit der Rechnung beeinflußt. Kassera [Kas97a] stellte fest, daß dies wiederum aufwendige Variationsrechnungen notwendig macht. Ebenfalls nur testweise wurde das Verfahren COPLA [Cho95, Cho98] implementiert, das auch von dritter Ordnung genau und eine Weiterentwicklung von SMART [Gas88] ist. Es führt nicht zu besseren Ergebnissen als MLU, benötigt aber aufgrund der aufwendigen Berechnung der Quellterme deutlich längere Rechenzeiten und wurde deshalb wieder aus dem Code gestrichen.

Bei der Herleitung des MLU-Verfahrens [No192] werden dieselben Koeffizienten verwendet wie beim UPWIND-Verfahren, es treten lediglich Zusatzterme im Quellterm auf. Unter Verwendung der minmod-Funktion [No192]

$$
\operatorname{minmod}(A, B)= \begin{cases}A & \forall|A| \leq|B| \wedge A B>0 \\ B & \forall|A|>|B| \wedge A B>0 \\ 0 & \forall A B \leq 0\end{cases}
$$

erhält man z.B. für den top-Teilterm des Quellterms der $x$-Impulsgleichung

$$
\begin{aligned}
& -\Delta \xi \rho(\Delta u)_{t} u_{t}^{*}= \\
= & -\left[\left[-\Delta \rho u_{t}^{*}, 0\right]\right] \cdot \xi^{2} \cdot \operatorname{minmod}\left(\frac{u_{T T}-u_{P}}{4}, \operatorname{minmod}\left(u_{T}-u_{P}, u_{T T}-u_{T}\right)\right)- \\
& -\left[\left[\Delta \rho u_{t}^{*}, 0\right]\right] \cdot \xi^{2} \cdot \operatorname{minmod}\left(\frac{u_{T}-u_{B}}{4}, \operatorname{minmod}\left(u_{T}-u_{P}, u_{P}-u_{B}\right)\right)
\end{aligned}
$$


MLU ist dort von zweiter Ordnung genau, wo in (2-34) $A B>0$ ist, ansonsten degeneriert es zu einem Verfahren erster Ordnung. MLU hat sich als wesentlich am leistungsfähigsten aller vier im Code verbliebenen Schemata herausgestellt. Die untersuchten Verfahren erster Ordnung waren nicht in der Lage, für die Kräfteentstehung an den Wärmetauscherrohren wesentliche Strömungsphänomene, insbesondere Wirbelablösung, bei höheren Reynoldszahlen korrekt darzustellen. Daher wurde MLU als einziges verbliebenes Verfahren höherer Ordnung innerhalb der vorliegenden Arbeit standardmäßig für die Berechnungen an Einzelrohren und realen Rohrbündeln verwendet.

\subsubsection{Lösung des Gleichungssystems}

\subsubsection{Die Berechnung des Druckfeldes}

Die letzte freie Variable des Systems ist der Druck. Für den Druck steht keine weitere direkte Berechnungsgleichung zur Verfügung. Die Navier-StokesGleichungen als Impulsgleichungen ergeben diskretisierte Gleichungen für die einzelnen Geschwindigkeitskomponenten. Allerdings erfüllen die mit den Impulsgleichungen berechneten Geschwindigkeiten noch nicht die Kontinuitätsgleichung. Daher wird das Druckfeld mit Hilfe einer diskretisierten Form der Massenerhaltungsgleichung so bestimmt, daß deren Erfüllung verbessert wird. Für die Berechnung des Druckfeldes bei Inkompressibilität werden in der Literatur im wesentlichen drei Möglichkeiten genannt:

- Einführung einer künstlichen Kompressibilität unter Verwendung der kompressiblen Kontinuitätsgleichung [Cho67a,b]. Dieses Verfahren ist jedoch nur für die Berechnung stationärer Strömungen geeignet [Fis95a] und scheidet daher für den vorliegenden Fall aus.

- Eine Differentialgleichung vom Poisson-Typ erhält man, wenn auf die Impulserhaltungsgleichungen in vektorieller Form der Divergenzoperator angewendet wird. Jedoch ist die Lösung der Gleichung sehr aufwendig, da sehr viele Terme in die Differentialgleichung eingehen, und schwierig zu stabilisieren [Schö90].

- Für das vorliegende Verfahren wurde daher eine Druckkorrekturgleichung aus den diskretisierten Impulsgleichungen und der diskretisierten Kontinuitätsgleichung abgeleitet [Oer95, No192, Schö90].

Die Herleitung der Geschwindigkeitskorrekturgleichungen wird wiederum am Beispiel der $x$-Impulsgleichung gezeigt. Die diskretisierte Form der Impulser- 
haltungsgleichungen im versetzten Gitter nach Abbildung 2-2 kann zunächst für die zu Beginn eines Iterationsschrittes vorliegenden Strömungsgeschwindigkeiten dargestellt werden als

$$
u_{P}^{*}\left(3 a_{P}^{0}+\sum a_{n b}\right)=\sum a_{n b} u_{n b}^{*}+4 a_{P}^{0} u_{P}^{n}-a_{P}^{0} u_{P}^{n-1}+S_{C}-\xi \Delta\left(p_{t}^{*}-p_{b}^{*}\right)
$$

wobei der Stern andeutet, daß es sich nur um geschätzte Größen handelt, und der Index $n b$ meint alle Nachbarknoten, die in der Impulsgleichung verwendet wurden. In der vorliegenden Herleitung ist also $n b \in\{T, B, E, W, N, S\}$, da bei der Diskretisierung ein Sieben-Knoten-Stern verwendet wurde. Für die exakte Lösung läßt sich die Gleichung analog formulieren. Man erhält

$$
u_{P}\left(3 a_{P}^{0}+\sum a_{n b}\right)=\sum a_{n b} u_{n b}+4 a_{P}^{0} u_{P}^{n}-a_{P}^{0} u_{P}^{n-1}+S_{C}-\xi \Delta\left(p_{t}-p_{b}\right)
$$

Die Differenz der beiden Gleichungen führt zu

$$
u_{P}^{\prime}\left(3 a_{P}^{0}+\sum a_{n b}\right)=\sum a_{n b} u_{n b}^{\prime}-\xi \Delta\left(p_{t}^{\prime}-p_{b}^{\prime}\right)
$$

Die gestrichenen Größen geben nun die Korrekturen an, die angebracht werden müssen, um von der geschätzten Lösung zur exakten Lösung zu gelangen. Der Quellterm entfällt, da er bei dem Korrekturschritt nicht verändert wird. Verschiedene Druckkorrekturverfahren unterscheiden sich in der Weise, wie die benachbarten Geschwindigkeitskorrekturen behandelt werden. Im SIMPLEVerfahren, das von Kassera auch verwendet wurde, werden alle benachbarten Geschwindigkeitskorrekturen zu Null gesetzt [Schö90, No193]. Dies führt dazu, daß zum einen die Nachbarkoeffizienten gespeichert werden müssen, zum anderen ist eine starke Relaxation notwendig. Eine bessere Nährung erhält man nach dem SIMPLEC-Verfahren, wenn man annimmt, daß alle Geschwindigkeitskorrekturen des betrachteten finiten Differenzensterns gleich groß sind [Schö90, No193]. Dies führt für die Geschwindigkeitskorrektur in $P$ zur Gleichung

$$
u_{P}^{\prime}=-\frac{\xi \Delta}{3 a_{P}^{0}}\left(p_{t}^{\prime}-p_{b}^{\prime}\right)
$$

Es müssen keine Koeffizienten mehr gespeichert werden und eine Relaxation ist nicht mehr notwendig. Die Konvergenzrate bei Anwendung des SIMPLECVerfahrens verdoppelt sich gegenüber der SIMPLE-Methode.

Die Kontinuitätsgleichung kann im nichtversetzten Kontrollvolumen nach Abbildung 2-3 in diskretisierter Form ebenso sowohl für die Schätzlösung 


$$
\frac{u_{P}^{*}-u_{B}^{*}}{\Delta x}+\frac{v_{P}^{*}-v_{W}^{*}}{\Delta}+\frac{w_{P}^{*}-w_{S}^{*}}{\Delta}=0
$$

als auch für die exakte Lösung

$$
\frac{u_{P}-u_{B}}{\Delta x}+\frac{v_{P}-v_{W}}{\Delta}+\frac{w_{P}-w_{S}}{\Delta}=0
$$

notiert werden. Die Differenz der beiden Gleichungen ergibt

$$
\begin{aligned}
& \xi\left(u_{P}^{\prime}-u_{B}^{\prime}\right)+\left(v_{P}^{\prime}-v_{W}^{\prime}\right)+\left(w_{P}^{\prime}-w_{S}^{\prime}\right)= \\
= & \xi\left(u_{P}^{*}-u_{B}^{*}\right)+\left(v_{P}^{*}-v_{W}^{*}\right)+\left(w_{P}^{*}-w_{S}^{*}\right)
\end{aligned}
$$

Für die Geschwindigkeitskorrekturgrößen der linken Seite können analog zu (2-39) die Beziehungen

$$
\begin{array}{rr}
u_{P}^{\prime}=-\frac{\xi \Delta}{3 a_{P}^{0}}\left(p_{T}^{\prime}-p_{P}^{\prime}\right) & u_{B}^{\prime}=-\frac{\xi \Delta}{3 a_{P}^{0}}\left(p_{P}^{\prime}-p_{B}^{\prime}\right) \\
v_{P}^{\prime}=-\frac{\Delta}{3 a_{P}^{0}}\left(p_{E}^{\prime}-p_{P}^{\prime}\right) & v_{W}^{\prime}=-\frac{\Delta}{3 a_{P}^{0}}\left(p_{P}^{\prime}-p_{W}^{\prime}\right) \\
w_{P}^{\prime}=-\frac{\Delta}{3 a_{P}^{0}}\left(p_{N}^{\prime}-p_{P}^{\prime}\right) & w_{S}^{\prime}=-\frac{\Delta}{3 a_{P}^{0}}\left(p_{P}^{\prime}-p_{S}^{\prime}\right)
\end{array}
$$

angegeben werden. Diese Korrekturgrößen können nun in die Kontinuitätsgleichung eingesetzt werden und führen auf ein lineares hauptachsendominantes Gleichungssystem für die Druckkorrekturen. In der Form eines Gauss-SeidelPoint-By-Point-Solvers ergibt sich etwa

$$
p_{P}^{\prime}=\frac{\xi^{2} p_{T}^{\prime}+\xi^{2} p_{B}^{\prime}+p_{E}^{\prime}+p_{W}^{\prime}+p_{N}^{\prime}+p_{S}^{\prime}+b}{2 \xi^{2}+4} .
$$

Dies entspricht einer gewichteten Mittelwertbildung der umliegenden Druckkorrekturgrößen. Es handelt sich daher um die diskretisierte Form einer Poisson-Gleichung. Dabei ist

$$
b=-\frac{3 a_{P}^{0}}{\Delta}\left\{\xi\left(u_{P}^{*}-u_{B}^{*}\right)+\left(v_{P}^{*}-v_{W}^{*}\right)+\left(w_{P}^{*}-w_{S}^{*}\right)\right\}
$$

der Massenerhaltungsfehler einer nichtversetzten Zelle bei geschätztem Strömungsfeld. Die zunächst geschätzten Geschwindigkeiten können dann bei vorliegenden Druckkorrekturen nach (2-43) verbessert werden. Dies verdoppelt etwa die Konvergenzrate. 


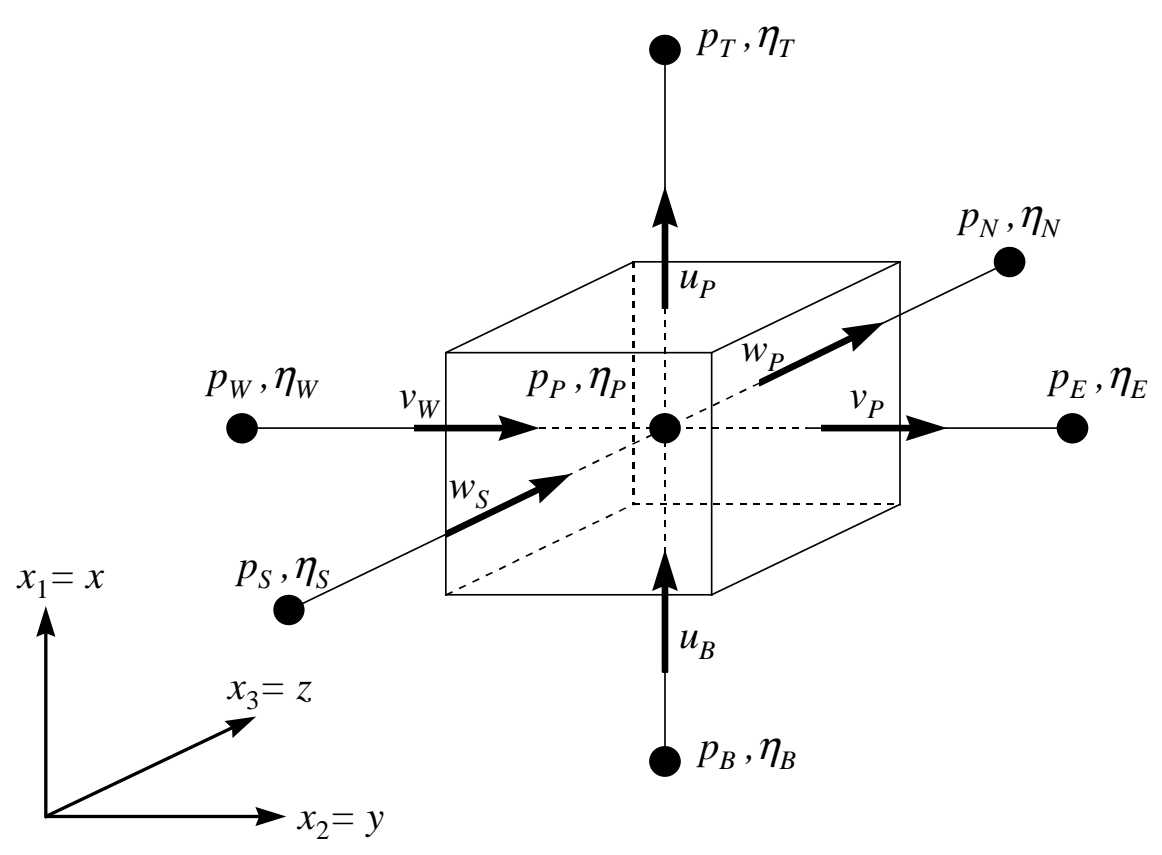

Abbildung 2-3: Nichtversetztes Kontrollvolumen.

Um die Berechnung an großen Modellen für die vorliegende Arbeit zu ermöglichen, mußten, wo immer möglich, Rechenzeit und Speicherplatz eingespart werden. Daher wurden in Rohrlängsachsenrichtung deutlich größere Zellkantenlängen verwendet als in den Hauptströmungsrichtungen senkrecht dazu. Physikalisch ist dies gerechtfertigt, da die bestimmende Größe für die Gefährdung hinsichtlich Rohrschwingungen die Queranströmkomponente ist. Die Vereinfachung führt aber dazu, daß die Druckverteilungen der einzelnen Hauptströmungsebenen nach der dargestellten Herleitung der Druckkorrekturgleichung numerisch nur sehr schlecht gekoppelt sind, da die top- und bottomKoeffizienten $\xi^{2}$ in der Druckkorrekturgleichung (2-44) wesentlich kleiner werden als Eins. Dies führt für ökonomische Zeitschritte und ökonomische Anzahlen von Iterationsschritten zu Instabilitäten, da das Druckfeld nicht ausreichend auskonvergiert wird. Daher wurden in der Druckkorrekturgleichung entgegen der exakten Herleitung alle Koeffizienten gleich Eins gesetzt. Es ergibt sich schließlich die auch für alle Berechnungen in der vorliegenden Arbeit verwendete Druckkorrekturgleichung

$$
p_{P}^{\prime}=\frac{p_{T}^{\prime}+p_{B}^{\prime}+p_{E}^{\prime}+p_{W}^{\prime}+p_{N}^{\prime}+p_{S}^{\prime}+b}{6}
$$


Das Vorgehen ist gerechtfertigt, da es sich ja nur um eine Korrektur-Gleichung handelt, nicht um eine Gleichung zur direkten Berechnung einer Größe. Die tiefere Problematik der langsamen Konvergenz des Druckfeldes liegt darin, daß es sich lediglich um eine Diffusionsgleichung handelt, bei der die Randbedingungen eben nur aufgrund diffusiver Vorgänge in das Berechnungsgebiet getragen werden. Der größte Teil der Berechnungszeit entfällt daher bei entkoppelten Verfahren auch auf die Berechnung des Druckfeldes [Schö90]. Um ein Überlaufen des Druckfeldes zu verhindern, werden nach jedem Rechenschritt alle Druckwerte auf den zentralen Druckwert im Auslaß bezogen. Für inkompressible Rechnung ist dies zulässig, da für die Strömungsfeldberechnung nur Druckdifferenzen relevant sind.

Ein solches Vorgehen zur Beschleunigung der Berechnung ist bei den Impulsgleichungen nicht notwendig, da aufgrund des konvektiven Charakters der Gleichungen die Randbedingungen ohnehin sehr viel schneller in das Berechnungsgebiet getragen werden. Außerdem würde die Fortlassung des Streckungsfaktors zu falschen Ergebnissen führen, da die entsprechenden Gleichungen ja der direkten Berechnung von Strömungsgrößen und nicht nur einer Korrektur dienen.

\subsubsection{Strömungsrandbedingungen}

Als Randbedingungen für die Impulsgleichungen werden am Eintritt und Austritt die Geschwindigkeiten in $y$-Richtung gesetzt. Dabei wird eine zeitabhängige Rampenfunktion zur Simulation des Hochlaufvorganges der Strömung verwendet. In $x$ - und $z$ - Richtung wird am Eintritt die Geschwindigkeit zu Null vorgegeben, am Austritt werden modifizierte Impulsgleichungen zur Berechnung verwendet, bei denen aufgrund der Annahme eines parabolischen Strömungsverhaltens die stromabwärts gelegenen Koeffizienten der diskretisierten Impulsgleichungen zu Null gesetzt werden. An festen Wänden wird die Geschwindigkeit Null, an bewegten Wänden die Wandgeschwindigkeit entsprechend der Haftbedingung der Strömungsmechanik vorgegeben.

Bei Simulationen von Kanalströmungen stellt das große Streckverhältnis $1 / \xi$ in Rohrlängsachsenrichtung kein Problem für die Aufgabe der Randbedingungen dar. Bei der Simulation von Wärmetauschermodellen hingegen wird die Geometrie der $\mathrm{Zu}$ - und Abströmstutzen durch treppenförmige Polyederflächen dargestellt. Welche Zellen als Eintritts- oder Austrittszellen gewählt werden, wird anhand der Ähnlichkeit der Zellenanordnung zu einem kreisrunden Stutzen- 
querschnitt und anhand der Massenerhaltung entschieden. Dabei wird unterschieden zwischen Randzellen und Zentralzellen. Zentralzellen werden mit der sich analytisch ergebenden Stutzenströmungsgeschwindigkeit belegt, während die Randzellen mit einer etwas kleineren Geschwindigkeit belegt werden, die so gewählt ist, daß die Summation aller Eintritts- bzw. Austrittsmassenströme auf den Eintritts- bzw. Austrittszellen gleich dem vorgegebenen Massenstrom ist. Die von der Kreisform abweichende Form des Zustromquerschnitts wirkt sich nicht auf die Berechnungsergebnisse hinsichtlich Schwingungsgefährdung aus. Kassera [Kas94] hat experimentell festgestellt, daß nicht die Form des Zustromstutzenquerschnitts (in seinen Versuchen quadratisch oder kreisförmig), sondern der Wert des eintretenden Massenstroms über das Schwingungsverhalten der Rohrbündel entscheidet. Als Randbedingungen für das Druckfeld wird die Druckkorrektur am Ausströmrand Null gesetzt [Schö90, No193].

\subsubsection{Solver-Techniken}

Alle Diskretisierungstechniken führen letztlich auf das Problem zurück, daß ein sehr großes, dünn besetztes lineares Gleichungssystem mit Bandstruktur gelöst werden muß [Schö90, No193, Fer99]. Im Falle der Druckkorrekturgleichung ist das System dabei symmetrisch, im Falle der Konvektionsgleichungen nicht. Statistische Untersuchungen am Programm haben ergeben, daß bei Verwendung des einfachsten Solvers ca. 60 \% der Laufzeit auf die Lösung der Gleichungssysteme entfällt, weitere ca. $20 \%$ auf die Berechnungen im Rahmen des LESTurbulenzmodells und nur ca. $20 \%$ auf die Berechnung der Koeffizienten des Systems. Der effizienten Lösung der linearen Gleichungssysteme kommt also große Bedeutung hinsichtlich der Wirtschaftlichkeit der Berechnung zu.

Der einfachste Solver ist der Gauss-Seidel-Point-By-Point-Solver, der auch von Kassera [Kas97a] verwendet wurde. Die Lösung an einem Gitterpunkt ergibt sich dabei durch gewichtete Mittelwertbildung aus den umliegenden Lösungswerten. Die Gewichtungsfaktoren ergeben sich aus der Diskretisierung der Erhaltungsgleichungen.

Der Solver hat nur eine mäßige Konvergenzrate und wurde daher bald durch einen Line-By-Line-ADI-Solver [Schö90, Pre86] ersetzt. Das Gleichungssystem wird dazu so umgeschrieben, daß ein tridiagonales System entsteht, das mit dem Thomas-Algorithmus [Pre86] gelöst werden kann. Das Strömungsfeld wird dabei Linie für Linie durchlaufen, die Durchlaufrichtungen werden bei jedem Durchgang permutiert. Die Kopplung mit den Randbedingungen wird dadurch 
verbessert. Dies führt dazu, daß die Konvergenzrate gegenüber dem Point-ByPoint-Solver etwa verdoppelt werden konnte.

In der Literatur [Schö90, Fer99] sind weitere Verfahren beschrieben, etwa Konjugierte-Gradienten-Verfahren, die Lower-Upper-Dekompositionen von Stone oder Multigrid-Solver. Von diesen Verfahren wurden der SIP3DAlgorithmus, der ICCG-Algorithmus und der CGSTAB-Algorithmus implementiert [Fer99]. SIP3D und CGSTAB sind für unsymmetrische und symmetrische Systeme, ICCG nur für symmetrische Systeme geeignet. CGSTAB kann bei einer Null-Startlösung zu Beginn der Rechnung mit dem entwickelten Code nicht verwendet werden, da sich Divisionen durch Null ergeben. Abhilfe schafft eine Vorbelegung des Druckfeldes, z.B. mit Atmosphärendruck oder einem beliebigen von Null verschiedenen Druckwert.

Es hat sich daher als vorteilhaft erwiesen, einen Berechnungslauf mit NullStartbelegung mit SIP3D oder ADI zu starten und nach einigen Zeitschritten das Druckfeld mit ICCG oder CGSTAB und die Konvektionsgleichungen mit CGSTAB zu lösen. Gegenüber ADI ergibt sich noch einmal etwa eine Verdreifachung der Konvergenzrate. Das Programm verfügt also über vier SolverAlgorithmen: ADI, SIP3D, ICCG und CGSTAB, wodurch die Konvergenzrate gegenüber dem von Kassera verwendeten Point-By-Point-Solver insgesamt etwa versechsfacht werden konnte.

Multigrid-Verfahren wurden nicht implementiert. Sie können ihre Vorteile v.a. bei stationären Rechnungen oder bei diffusionsdominierten Differentialgleichungen ausspielen [Schö90]. Bei Multigrid-Verfahren wird der numerische Fehler zunächst auf einem groben Gitter geglättet. Die Lösungen werden schrittweise bis auf das feinste Gitter weiterinterpoliert. Da im vorliegenden Fall instationär gerechnet wird, liegt für jeden Zeitschritt aber ohnehin bereits eine sehr gute Startlösung vor, nämlich die Lösung des vorhergehenden Zeitschritts. Während bei stationären Rechnungen die Rechenzeiten um Faktoren in der Größenordnung von 100 reduziert werden können, ist dies bei instationären Rechnungen nur etwa um einen Faktor 2 möglich [Schö90]. Dabei steigt jedoch der Programmieraufwand leicht um ca. $100 \%$ an, der Speicherbedarf steigt noch stärker, und zwar in dem Maße, indem die Anzahl der Rechennetze erhöht wird [Schö90].

Die einzige Gleichung, die im vorliegenden Fall sinnvoll mit einer MultigridTechnik zu lösen wäre, ist die Druckkorrekturgleichung. Schönung verweist jedoch darauf [Schö90], daß sich die Konvergenzrate des entkoppelt rechnen- 
den Gesamtverfahrens nur minimal verbessern läßt. Theodossiou und Sousa [The86] stellen darüber hinaus eine nachlassende Genauigkeit bei höheren Reynoldszahlen fest. Ein Multigrid-Verfahren zu implementieren erschien daher im vorliegenden Falle nicht ökonomisch.

\subsubsection{Ablauf der strömungsmechanischen Berechnung}

Aus den dargestellten diskretisierten Berechnungsgleichungen für Geschwindigkeiten, Druck und Zähigkeit ergibt sich folgendes Iterationsschema für die strömungsmechanische Berechnung:

1. Es liegt ein geschätztes Druck- und Geschwindigkeitsfeld vor. Bei Rechnungsbeginn sind alle Größen Null, im weiteren Verlauf wird das Strömungsfeld vom letzten Iterationsschritt als Startlösung verwendet.

2. Berechnung der lokal optimierten Smagorinsky-Konstante und daraus Berechnung der turbulenten Viskosität.

3. Berechnung der Geschwindigkeiten aus den Impulsgleichungen.

4. Berechung der Druckkorrekturen.

5. Aktualisierung des Druckfeldes.

6. Verbesserung des Geschwindigkeitsfeldes.

7. Weiter mit 2., bis die vorgegebene Anzahl von Iterationen bzw. das vorgegebene Residuum der Kontinuitätsgleichung innerhalb des Zeitschritts erreicht ist.

8. Aktualisierung der alten Strömungsfelder durch „Weiterschieben“ der Datenfelder um eine Zeitebene. Beginn des neuen Zeitschritts mit 2.

Im Rahmen der fluidstrukturgekoppelten Rechnung wird dieser Berechnungsablauf noch erweitert werden.

\subsubsection{Testberechnungen: Kräfte auf einen umströmten Einzelzylinder}

Um das numerische Berechnungsverfahren hinsichtlich der Umströmung zylindrischer Körper überprüfen zu können, wurden Testberechnungen der Strömungskräfte für starre Einzelzylinder im Reynolds-Zahlenbereich von 2000 bis 200000 durchgeführt, bei denen die sich durch Summation aller Oberflächendrücke und Oberflächenscherkräfte ergebenden Kräfte auf die Rohre überprüft wurden. Die in den Messungen für die vorliegende Arbeit verwendeten Reynoldszahlen lagen, auf das Einzelrohr bezogen, zwischen etwa 20000 und 65000, so daß die durchgeführte Parametervariation für den Einzelzylinder den real zu erfassenden Reynoldszahlenbereich großzügig abdeckt. 
Um die Strömungskräfte hinsichtlich ihres Betrages dimensionslos machen zu können, werden der Widerstandsbeiwert

$$
c_{W}=\frac{2 F_{y}}{\rho D L u_{0}^{2}}
$$

und der Auftriebsbeiwert

$$
c_{A}=\frac{2 F_{z}}{\rho D L u_{0}^{2}}
$$

definiert, die die Strömungskräfte in Anströmungsrichtung bzw. quer zur Anströmungsrichtung in Beziehung setzen zum Staudruck und der Projektion der angeströmten Zylinderfläche in Strömungsrichtung. Infolge von Wirbelablösungen schwanken beide Beiwerte zeitlich. Für den Vergleich mit Meßergebnissen wird nachfolgend für den Auftrieb der maximale Beiwert sowie für den Widerstand der mittlere Beiwert verwendet. Die Reynoldszahl wird auf den Zylinderaußendurchmesser $D$ bezogen. Zur Charakterisierung der Wirbelablösefrequenz wird die Strouhalzahl

$$
S r=\frac{f_{W} D}{u_{0}}
$$

verwendet, die die Wirbelablösefrequenz $f_{W}$ dimensionslos darstellt.

Als Ausgangsgitter für die numerische Simulation wurde die Diskretisierung eines Kanals mit 10 x 108 x 36 Zellen gewählt. Die 10 Zellen in $x$-Richtung waren dabei $4 \mathrm{~mm}$ lang, um eine zu große Beeinflussung der Widerstandsbeiwerte infolge von Grenzschichteffekten an den oberen und unteren Kanalwänden zu verhindern. In $y$ - und $z$-Richtung wurden $2 \mathrm{~mm}$ als Kantenlänge verwendet, so daß die Wirbelablösungen in den Hauptströmungsebenen aufgelöst werden konnten. Der umströmte Zylinder hatte $12 \mathrm{~mm}$ Durchmesser. Der Durchmesser wurde also im Ausgangsgitter durch 6 Zellen dargestellt. Der Zylinder war bei 1/6 der Kanallänge positioniert. Dadurch war ein ausreichender Abstand vom Abströmrand gewährleistet. Der Kanal war außerdem breit genug, um keine künstliche Erhöhung der Wirbelablösefrequenz durch eine zu große Versperrung zu erzeugen [Che99]. Als Gitterverfeinerung wurde ein Gitter mit 10 x 150 x 50 Zellen bei sonst gleicher Geometrie verwendet.

Für Widerstandsbeiwerte $c_{W}$ und Strouhalzahlen $S r$ gibt es eine große Anzahl von Meßwerten in der Literatur. Gute Übersichtsbeiträge hierzu liefern Cant- 
well und Coles [Can83], Chen [Che73], Lienhard [Lie66] und Drescher [Dre56], die Meßwerte für den Reynoldszahlenbereich von 1 bis $10^{7}$ zusammengetragen haben. In Abbildung 2-4 und Abbildung 2-5 werden die in der Literatur angegebenen Meßwerte durch eine obere und eine untere Einhüllende dargestellt. Die numerisch berechneten Werte für verschiedene Reynoldszahlen sind durch einzelne Datenpunkte dargestellt.

Die Literaturwerte für den Auftriebsbeiwert $c_{A}$ sind weit stärker gestreut als diejenigen für Widerstandsbeiwert und Strouhalzahl. Chen [Che73] stellt eine Reihe von experimentellen Ergebnissen zusammen. Die in Abbildung 2-6 eingezeichnete obere Begrenzung des Streubandes stellt eine theoretische Begrenzung für die Auftriebsbeiwerte dar. Die untere Begrenzung stellt die kleinsten gemessenen Meßwerte nach [Che73] und [Jen82] dar. Alle bekannten Meßwerte liegen nach [Che73] und [Jen82] zwischen diesen beiden Einhüllenden. Die mit dem Programm in der vorliegenden Arbeit numerisch berechneten Auftriebsbeiwerte sind als Datenpunkte ebenfalls verzeichnet.

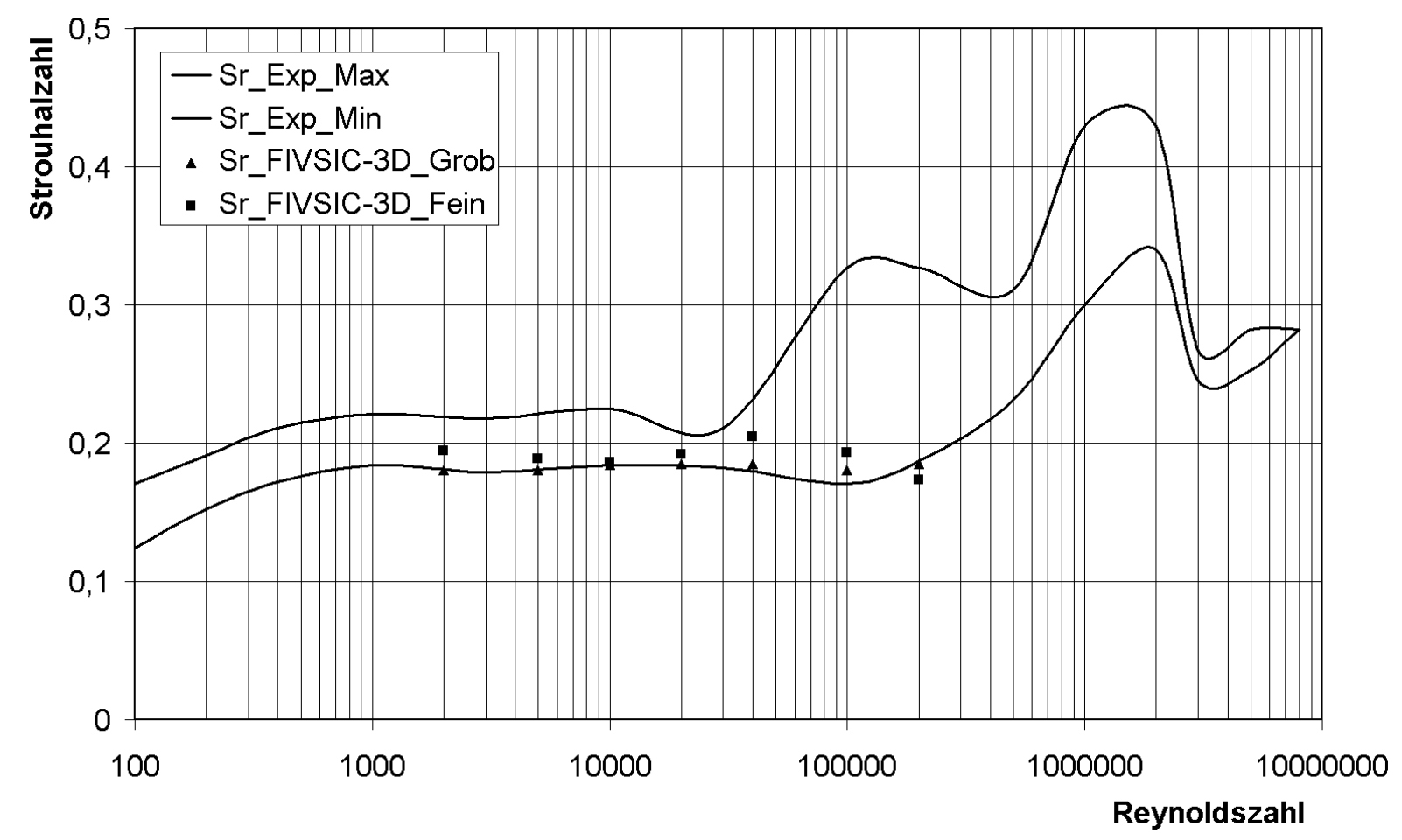

Abbildung 2-4: Vergleich zwischen numerischer Rechnung und Meßwerten der Literatur für die Strouhalzahl des starren Einzelzylinders. 


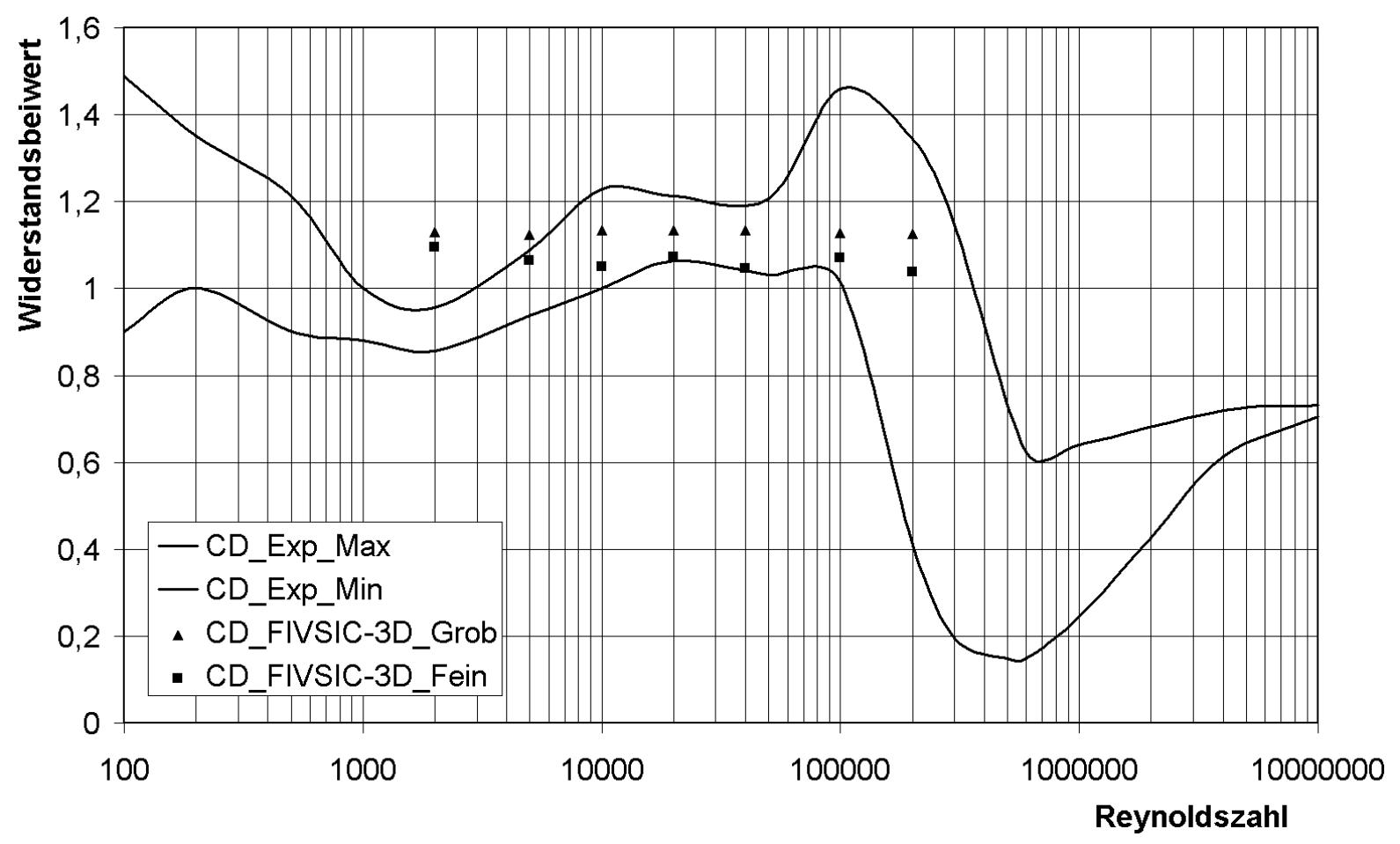

Abbildung 2-5: Vergleich zwischen numerischer Rechnung und Meßwerten der Literatur für den Widerstandsbeiwert des starren Einzelzylinders.

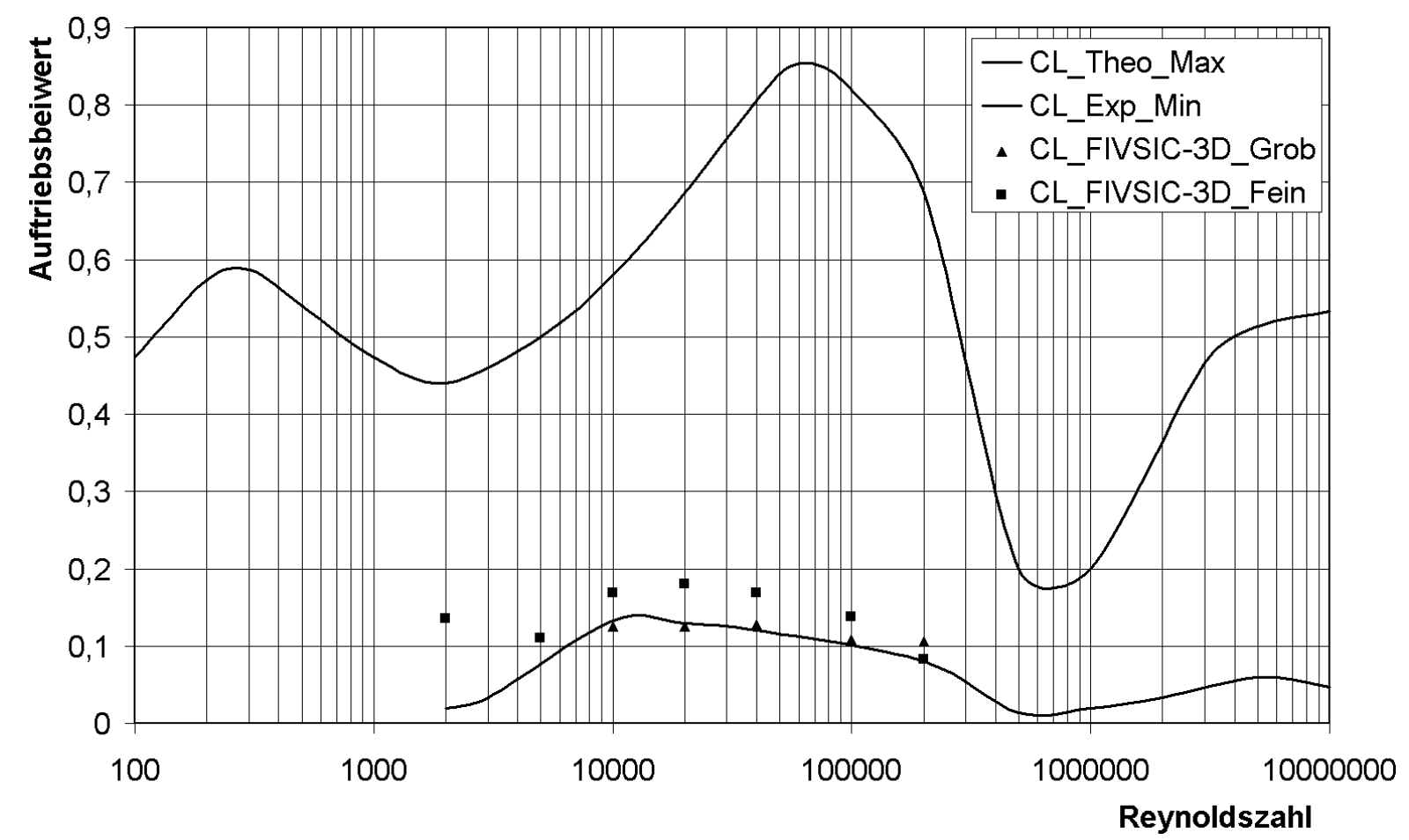

Abbildung 2-6: Vergleich zwischen numerischer Rechnung und Meßwerten der Literatur für den Auftriebsbeiwert des starren Einzelzylinders. 
Wie man sieht, liegen die vom numerischen Verfahren berechneten Werte für Widerstandsbeiwert und Strouhalzahl innerhalb der Bandbreite der von der Literatur zur Verfügung gestellten Meßwerte. Lediglich der Abfall des Widerstandsbeiwertes bei kleinen Reynoldszahlen wird nicht korrekt erfaßt. Dieses Verhalten stellte Kassera [Kas97a] auch bei seinem Simulationscode fest. Die berechneten Strouhalzahlen liegen im unteren Bereich der Meßwerte, aber immer noch innerhalb des Toleranzbandes. Mit zunehmender Auflösung steigt der berechnete Auftriebsbeiwert an. Die berechneten Auftriebsbeiwerte liegen im Bereich der unteren Begrenzung, fallen aber alle in das Toleranzband der veröffentlichten Meßwerte hinein. Das Modell zur Berechnung der strömungsmechanischen Kräfte konnte somit für die vorliegende Arbeit validiert werden.

\subsubsection{Alternative Diskretisierungstechniken}

\subsubsection{Die Finite Analytische Methode}

Eine sehr genaue Methode zur numerischen Lösung partieller dreidimensionaler Konvektions-Diffusions-Differentialgleichungen ist die Finite Analytische Methode, die maßgeblich von Chen et al. seit etwa 1980 entwickelt wurde [Che88, Che95, Suh89]. Da sie in der Standardliteratur fast nicht behandelt wird, soll sie als Alternative zu dem dargestellten Verfahren näher beleuchtet werden.

Bei der Finiten Analytischen Methode werden die Navier-Stokes-Gleichungen innerhalb eines Kontrollvolumens linearisiert und diese linearisierten Transportgleichungen mit Hilfe von stetigen, monotonen Randfunktionen exakt gelöst, die sich aus umliegenden Werten ergeben, die bereits als bekannt angenommenen werden und die partiellen Differentialgleichungen bereits für sich erfüllen [Suh89]. Die Gesamtheit aller Teillösungen stellt die Gesamtlösung der partiellen Differentialgleichungen in dem komplex berandeten Gebiet dar und kann iterativ (z.B. durch Gauss-Seidel-Verfahren mit sukzessiver Unterrelaxation SUR) bestimmt werden. Um den Rechenaufwand gering zu halten, wird für die zeitliche Diskretisierung eine Vorwärts-Finite-Differenz verwendet. Man spricht daher von einem hybriden Finiten Analytischen Verfahren. Die Lösung ist stabil und konvergent [Zen87] und beinhaltet einen automatischen UPWIND-Effekt [Che95], der die Forderung nach der Erhaltung der Transporteigenschaften der diskretisierten Transportgleichungen erfüllt. 
Die vollständige Finite Analytische Lösung verknüpft 28 benachbarte Knoten (27 räumliche Knoten und eine weitere Zeitebene) miteinander und stellt damit bezüglich der unmittelbar benachbarten räumlichen Knoten die genauestmögliche Diskretisierung der Transportgleichung dar, wobei die schlechte zeitliche Diskretisierung die Lösung aber i.A. kontaminiert. Die Koeffizienten der algebraisierten Ergebnisformeln für die Transportgrößen beinhalten unendliche Reihen und eine Anzahl von exponentiellen und hyperbolischen Funktionen. Chen weist zwar nach, daß eine von ihm hergeleitete 20-Knoten-Formel, die aus einer Superposition von zweidimensionalen Lösungen entsteht, numerisch fast dieselben guten Ergebnisse liefert wie die 28-Knoten-Formel. Aber auch die vereinfachte Finite Analytische Lösung enthält eine unendliche Reihe. Chen beschreibt zwar, daß sich in der Praxis die Berechnung von 20 Gliedern als ausreichend erwiesen habe, dennoch benötigt die Auswertung der Koeffizienten für beide Lösungsansätze eine hohe Rechenzeit [Che95].

Bei höheren Zell-Peclet-Zahlen gibt es zudem numerische Schwierigkeiten bei der Auswertung der Finiten Analytischen Koeffizienten. Es wurden verschiedene Methoden entwickelt, diese Probleme zu umgehen.

Eine Möglichkeit ist die Verwendung eines hybriden elliptisch-parabolischen Ansatzes. Bei höheren Zell-Peclet-Zahlen wird der Einfluß der Diffusion immer geringer. Deshalb soll die Finite Analytische Lösung von Chen nur in den Bereichen der Grenzschichten, der Nachlaufwirbel und anderen Gebieten, in denen die Diffusion vorherrschend ist, verwendet werden, dort aber vollständig. In den Gebieten mit vorherrschender Konvektion soll die schnellere parabolisierte Finite Analytische Lösung Anwendung finden.

Für höhere Zell-Peclet-Zahlen kann der Reibungsterm in den Navier-StokesGleichungen vernachlässigt werden. Er wird zunächst dem Quellterm zugeschlagen und als konstant angesehen. Die entstehende partielle Differentialgleichung erster Ordnung ist parabolisch. Mit Hilfe von stromauf gelegenen Knotenwerten und der Charakteristiken-Methode [Suh89, Dal83] kann dann eine UPWIND-Finite-Analytische Lösung für das entstandene Cauchysche Anfangswertproblem gefunden werden. Danach werden die Reibungsterme mittels Finiter Differenzen dargestellt. Es entsteht eine 28-Knoten-Formel, die UPWIND-Eigenschaften hat, aber auch Querdiffusionseffekte beinhaltet [Fis95b]. Sie erfüllt alle Forderungen von Patankar [Pat80] für die Stabilität einer Diskretisierung. Die Art der Lösung der Transportgleichung hat zur Folge, daß an Stellen, an denen aufgrund des hohen Diffusionsanteils eine erhöhte Genauig- 
keit gefordert wird (z.B. Grenzschicht, Staupunkte, Nachlaufwirbel, Ablösestellen etc.), auch eine gute Lösung zur Verfügung gestellt werden kann. Im höheren Zell-Peclet-Zahlbereich ist der UPWIND-Effekt vorherrschend, und die parabolisierte Finite Analytische Lösung kann angewendet werden [Fis95b].

Durch die hybride Verwendung der elliptischen und der parabolisierten Lösung wird auch Rechenzeit gespart, da die parabolisierte Lösung nur einen Bruchteil der Rechenzeit der vollständigen elliptischen Lösung verlangt [Fis95b]. Der Bereich der Anwendbarkeit der Lösung von Chen bestimmt sich durch die Art der verwendeten Maschine. Je größer die Maschinengenauigkeit, desto weiter der Nutzbarkeitsbereich. Trägt man die Versagenspunkte in einem dreidimensionalen Koordinatenkreuz auf, so erhält man eine dreidimensionale Fläche, deren Form und Ausdehnung von der Art der verwendeten Maschine abhängt.

Verschiedene Autoren versuchen außerdem, das Problem der numerischen Berechnung der Finiten Analytischen Koeffizienten des vollständigen elliptischen wie des parabolischen Ansatzes zu lösen. Dies ist jedoch entweder mit Genauigkeitsverlust verbunden [Suh89] oder mit einem hohen Aufwand [Zha92].

Bei größeren Werten der Zell-Peclet-Zahlen entsteht bei der numerischen Auswertung der Koeffizienten der Finiten Analytischen Methode das Problem des Überlauffehlers, da Quotienten sehr großer Zahlen zu bilden sind. Suh [Suh89] verwendet in seiner Arbeit eine Approximation für $\exp (-x)$ mit $x>0$, die Hastings [Has55] 1955 angegeben hat. Wird darin $x$ durch $-x$ ersetzt, erhält man

$$
\begin{aligned}
& e^{x}=\frac{1}{\left(1-A_{1} x+A_{2} x^{2}-A_{3} x^{3}+A_{4} x^{4}-A_{5} x^{5}+A_{6} x^{6}\right)^{4}} \\
& A_{1}=0,2499986842 \quad A_{4}=0,0001715620 \\
& A_{2}=0,0312575832 \quad A_{5}=0,0000054302 \\
& A_{3}=0,0025913712 \quad A_{6}=0,0000006906
\end{aligned}
$$

für $x<0$. Für $x>0$ kann $\exp (x)$ bestimmt werden, wenn der Kehrwert der Funktion von Hastings gebildet wird, und man erhält

$$
e^{x}=\left(1+A_{1} x+A_{2} x^{2}+A_{3} x^{3}+A_{4} x^{4}+A_{5} x^{5}+A_{6} x^{6}\right)^{4}
$$

Dabei werden dieselben $A_{i}$ verwendet. Im Bereich $|x|<10$ liegt der Fehler unter $0,5 \%$. Für $x<-10$ werden die sehr kleinen Werte $\exp (x)$ zu groß, für $x>10$ dagegen die sehr großen Werte zu klein. Damit wird der Überlauf auch bei gro- 
Ben $x$ verhindert. Im Bereich um $x \approx 0$ ist dagegen die Genauigkeit gut. Die Formel ist damit geeignet, die Koeffizienten der Finiten Analytischen Methode näherungsweise zu bestimmen. Im Bereich der überwiegenden Diffusion werden die Koeffizienten sehr genau berechnet, im Bereich der überwiegenden Konvektion genau genug, um den UPWIND-Effekt der FAM beizubehalten. Jedoch ergeben sich aufgrund der großen Anzahl von Rechenoperationen hohe Rechenzeiten.

In [Zha92] werden die Koeffizienten mit einem externen Algebra-Programm genau berechnet und tabelliert. Die Tabelle wird später zur Interpolation von Koeffizienten herangezogen. Das Programm Mathematica [Wo197, Lad98, Fis00b] ist ein solches externes Algebraprogramm, das auf beliebig viele Stellen genau rechnen kann. Bei dem Versuch, Finite Analytische Koeffizienten damit zu bestimmen, konnte jedoch in bezug auf die benötigte Rechenzeit kein zufriedenstellendes Ergebnis erreicht werden. Auch andere Ansätze zur Erhöhung der Rechengenauigkeit wurden untersucht, z.B. [Aut89]. Keiner dieser Ansätze stellte einen wirklich praktikablen Weg zur Bestimmung von KoeffizientenTabellen dar.

Zusammenfassend ergeben sich damit für höhere Zell-Peclet-Zahlen die folgenden Möglichkeiten zur Lösung der Transportgleichungen mit Hilfe der Finiten Analytischen Methode [Fis95b]:

1. Verwendung der elliptischen Finiten Analytischen Lösung von Chen unter Verwendung von Built-In-Funktionen bis zur Stabiltätsgrenze der ZellPeclet-Zahl, danach Verwendung der parabolisierten Finite-ElementeLösung.

2. Wie 1., jedoch mit Hilfe der approximierten Exponentialfunktion nach [Suh89] und [Has55]. Dadurch kann der Bereich der Zell-Peclet-Zahlen nahezu verdoppelt werden.

3. Wie 1., jedoch Berechnung der Koeffizienten mit Hilfe vorher berechneter und tabellierter Stützwerte in Abhängigkeit der Konvektion in die drei Raumrichtungen.

Ein vollständiges Verfahren nach der Finiten Analytischen Methode wurde in [Fis95b] abgeleitet. Beide Formelapparate von Chen verwenden ein orthogonales und für jede Raumrichtung jeweils äquidistantes Gitter. In [Fis95b] wird gezeigt, daß die Formelapparate mit Hilfe von Koordinatentransformationen auch für krummlinige Koordinatensysteme nutzbar sind. In [Che95] wird der Stand der Wissenschaft auf dem Gebiet der FAM um 1995 mitgeteilt. 
Für die vorliegende Arbeit wurde die Methode aus den folgenden Gründen letztlich verworfen:

- Die Methode macht abhängig von der Zell-Peclet-Zahl ein Umschalten zwischen parabolischem und elliptischem Ansatz notwendig. Ein Umschalten entspricht jedoch programmiertechnisch einer Abfragebedingung, was den Simulationsablauf verlangsamt und insofern keinen Vorteil gegenüber UPWIND-Ansätzen der Finite-Volumen-Methode darstellt. Außerdem wird ein sehr aufwendiger Programmcode benötigt.

- Es müssen sehr viel mehr Rechenoperationen zur Bestimmung der Koeffizienten der diskretisierten Transportgleichungen durchgeführt werden als z.B. bei der FVM oder der FD, was die Ausführungszeit deutlich erhöht.

- Bei Verwendung von Approximationsfunktionen für die zu berechnenden transzendenten Funktionen wird gerade der Vorteil der hohen Genauigkeit der Methode wieder zunichte gemacht.

- Bei Verwendung von tabellierten Koeffizienten ergeben sich aus den Interpolationen ebensolche Ungenauigkeiten. Für sehr viel höhere Genauigkeiten müßten aufwendig zu berechnende und speicherplatzintensive Tabellen vorab bestimmt und gespeichert werden.

- Sowohl die Herleitung als auch die Programmierung der Methode sind aufwendig und fehleranfällig.

- Die Methode wurde bisher nur für kleine Reynoldszahlen verwendet. Ebenso ist in der Literatur nur wenig Erfahrung bei der Verwendung im Zusammenspiel mit Turbulenzmodellen dokumentiert.

Die Finite Analytische Methode stellt einen wissenschaftlich interessanten Ansatz als Konkurrenz zu FD, FEM und FVM dar und hat im Bereich kleiner Reynoldszahlen bereits beachtliche Erfolge vorzuweisen. Im Hinblick auf den innerhalb dieser Arbeit zu simulierenden Reynoldszahlen-Bereich und die zu erwartenden komplexen Phänomene innerhalb des dreidimensionalen instationären Strömungsfeldes scheint die Finite Analytische Methode aber noch nicht ausgereift genug zu sein, um für ein Problem der Klasse der 3D-Rohrbündeldurchströmungen bei bewegten Rohren schon anwendbar zu sein. Sollten sich jedoch die Probleme im Zusammenhang mit der Berechnung der Koeffizienten der diskretisierten Transportgleichungen von den entsprechenden Arbeitsgruppen lösen lassen, könnte hier für die vorliegende Problemstellung eine leistungsfähige Methode entstehen, da das innerhalb der vorliegenden Arbeit ent- 
standene Programm leicht um die Berechnung der Koeffizienten nach alternativen Methoden erweitert werden kann.

\subsubsection{Die Finite Elemente Methode}

Für die Finite Elemente Methode liegen zwar eine Reihe von Standardwerken vor (z.B. [Bat86, Zie84, Käm88]). Deshalb sollen hier die Grundlagen der Methode nicht nochmal wiederholt werden. Die Anwendung auf die numerische Strömungsmechanik kommt allerdings erst in neuerer Zeit voran [Chu83]. Gegen Überlegungen, das Simulationsprogramm in der vorliegenden Arbeit nach der Finite-Elemente-Methode zu programmieren, sprachen im wesentlichen die folgenden Gründe:

- Die Finite-Elemente-Methode arbeitet mit unstrukturierten Gittern. Für den Fall, daß sich Rohre sehr nahe kommen, muß das Gitter nach jedem Rechenschritt neu ermittelt werden. Dies führt zu erhöhter Rechenzeit.

- Durch die lokal nicht kartesischen Koordinaten entstehen wie bei körperangepaßten Koordinaten bei der Finite-Volumen-Methode Kreuzterme und Coriolisterme, die die Konvergenzrate verringern.

- Die automatische Vernetzung ist beim heutigen Stand der Technik nur für Tetraeder-Gitter möglich. Gerade diese zeichnen sich aber im Rahmen der FEM durch relativ große Ungenauigkeiten aus. Eine automatische Vernetzung ist aber heute eine der Forderungen, die an numerische Verfahren gestellt wird.

- Für die Anwendung höherer Diskretisierungsschemata für die konvektiven Terme liegen noch relativ wenige Erfahrungswerte vor. Die im Rahmen der vorliegenden Arbeit getesteten hochwertigen Schemata MLU und COPLA sind selbst für die FVM in ihrem Verhalten noch relativ unerforscht. Im Standardwerk von Chung [Chu83] wird schon die UPWIND-Diskretisierung mit FEM als relativ aufwendig und problematisch dargestellt.

- Die Anwendung von Turbulenzmodellen im Rahmen der FEM ist noch nicht sehr weit verbreitet und stellte insofern eine weitere Unbekannte für das zu entwickelnde Verfahren dar. Insbesondere für neuere dynamische Turbulenzmodelle liegen noch keine Erfahrungswerte im Zusammenspiel mit FEM vor.

- Der Programmcode und die Speicherplatzanforderungen werden umfangreicher durch das Vorhalten der Buchhaltung der einzelnen Elemente und ihrer Verknüpfungen. 
Aufgrund der dargestellten Unwägbarkeiten und Komplikationen wurde der Gedanke eines FEM-Strömungssolvers als unpraktikabel verworfen.

\subsubsection{Analytische Behandlung der Spaltströmung}

In [Fis98b,c] wurde als industrielle Anwendung numerischer Strömungsmechanik die Einströmsektion eines Gaskühlers vollständig diskretisiert und das Strömungsfeld berechnet. Ziel war u.a. die Bestimmung des Druckverlustes der Einströmsektion. Aus Gründen des numerischen Aufwandes war es aber nicht möglich, die Auswirkungen der Spaltströmungen detailliert in ein globales Wärmetauschermodell zu integrieren, die Auswirkungen der Mitmodellierung der Spalte auf den Druckverlust wurden mit einem Einzelreihenmodell erfaßt [Fis99a]. Das Hauptproblem lag in dem Nebeneinander sehr kleiner und sehr großer geometrischer Abmessungen, die jedoch beide numerisch sachgerecht aufzulösen sind.

Um den Rechenaufwand gering zu halten und gleichzeitig die beschriebenen strömungsmechanischen Spalteffekte berücksichtigen zu können, war es wünschenswert, für solche Vorgänge analytische Formeln zur Verfügung zu haben, um nicht die strömungsmechanischen Vorgänge im Spalt auch noch numerisch mit den Methoden der CFD berechnen zu müssen. In [Pri98] wird im Rahmen eines Verfahrens zur numerischen Bestimmung des Gesamtdruckverlusts von Rohrbündelwärmetauschern für die Leckageströme ohne nähere Erklärung der Annahmen eine von der Spalt-Reynoldszahl abhängige halbempirische Formel verwendet, die auf der Verwendung einer modifizierten Bernoulli-Gleichung beruht. Hiermit ist es jedoch nicht möglich, bewegte Rohre zu berücksichtigen oder über die Strömungskräfte und die Strömungsverteilung im Spalt Aussagen zu treffen. Daher wurden in [Fis99a] analytische Formeln für die vorliegende Arbeit entwickelt, die die Berechnung von Geschwindigkeitsverteilungen im Spalt gestatten, um zu klären, ob die Spaltströmungen einen Einfluß auf die Rohrbündelschwingungen haben.

In [Fis99a] wird ein einphasiges, isothermes, inkompressibles Fluid der Dichte $\rho$ und der Zähigkeit $\eta$ in einem Ringspalt gemäß Abbildung 2-7 betrachtet. Durch eine radiale Verschiebung $\Delta w$ in Richtung des Winkels $\gamma$ entsteht zwischen dem Rohr mit Radius $r_{I}$ und der Bohrung mit Radius $R$ eine sichelförmige Querschnittsfläche. Das Rohr bewegt sich mit einer Geschwindigkeit $v_{R}$ in Richtung des im Allgemeinen von $\gamma$ verschiedenen Winkels $\beta$. Die Rohrlängsachse und die Oberflächennormale des Leitblechs der Dicke $s$ mögen dabei im- 
mer parallel zueinander stehen. Das kartesische Koordinatensystem habe seinen Ursprung im bewegten Rohrmittelpunkt. Diese Festlegung wird gewählt, um die spätere Berechnung der Kräfte auf das Rohr zu vereinfachen.

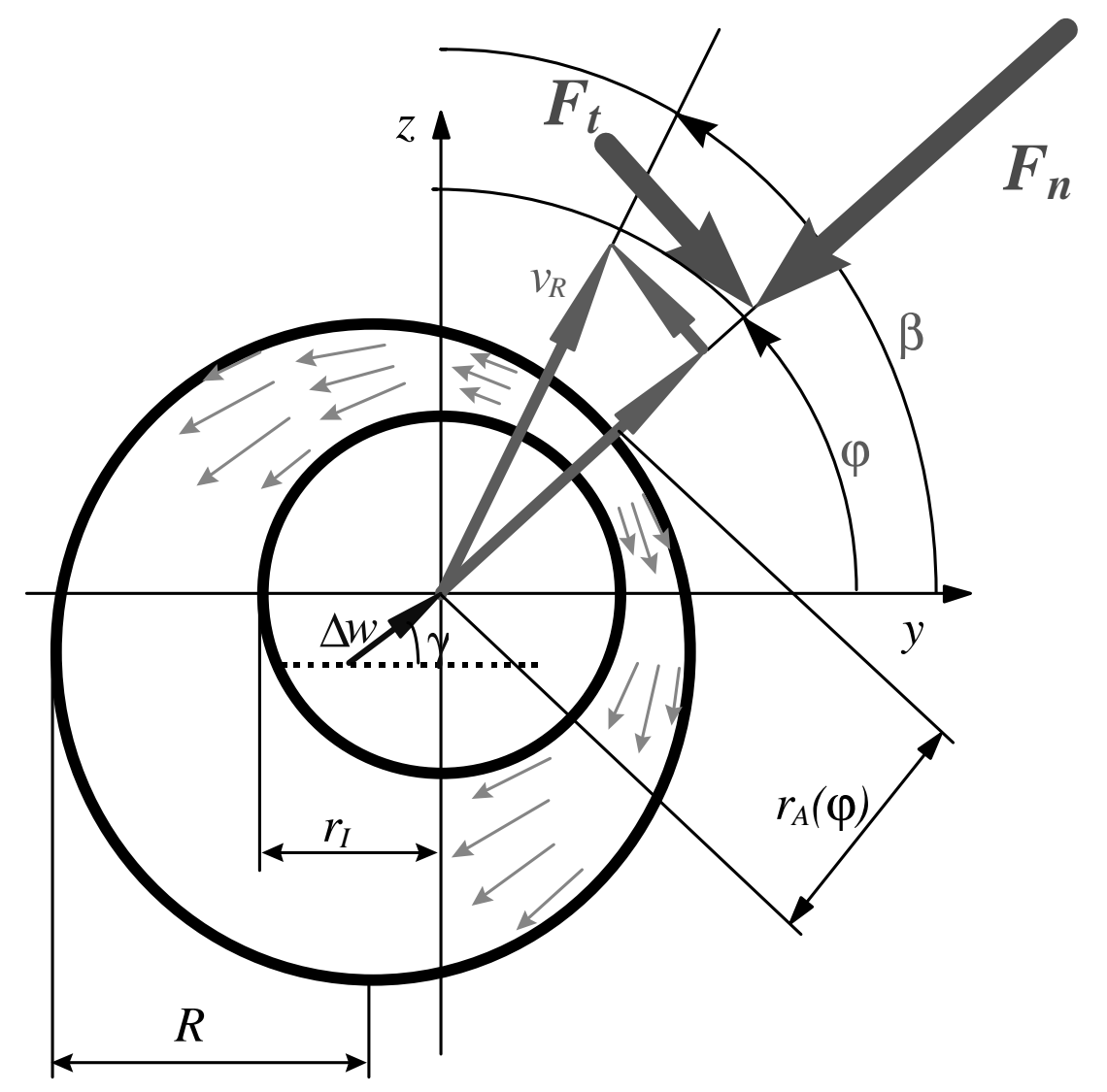

Abbildung 2-7: Geometrie des Ringspaltes zwischen Wärmetauscherohr und Leitblech bei bewegtem Wärmetauscherrohrrohr.

Zwischen der Unter- und der Oberseite des Leitblechs herrscht ein gewisser Druckunterschied $\Delta p$, der von der Rohrbewegung selber und den radialen Koordinaten unabhängig sein soll. Der Fluiddruck $p$ im Spalt soll sich in rohraxialer Richtung linear verhalten. Der Druckunterschied führt zu einem Druckgradienten, der wiederum eine rohraxiale Strömungskomponente $w_{x}(r, \varphi)$ antreibt. In den zur Rohrlängsachse senkrechten $y$-z-Ebenen durch den Spalt herrsche eine ebene Strömung mit den Geschwindigkeiten $w_{r}(r, \varphi)$ in radialer und $w_{\varphi}(r, \varphi)$ in azimutaler Richtung. Diese Modellannahme wird wegen der Randeffekte um so eher zutreffen, je größer die Leitblechdicke $s$ ist. Die Strömungen in Rohrlängsachsenrichtung und senkrecht dazu im Spalt seien voneinander entkoppelt. Die Strömungen werden aufgrund der kleinen geometrischen Maße des Spalts 
als laminar angenommen. Für die Leckageströmung in rohraxialer Richtung erhält man die Gleichung

$$
w_{x}(r, \varphi)=\frac{\Delta p}{4 s \eta} \cdot\left(r^{2}-r_{I}^{2}-\left(r_{A}^{2}-r_{I}^{2}\right) \frac{\ln \frac{r}{r_{I}}}{\ln \frac{r_{A}}{r_{I}}}\right)
$$

Das Auftreten eines nahezu parabolischen Geschwindigkeitsprofils über der Spaltbreite konnte mit Hilfe numerischer Berechnungen der Spaltströmungen mit dem CFD-Code CFX 4.1 [AEA97, Dra99] nachgewiesen werden. Allerdings ergeben sich bei zunehmender Auslenkung Abweichungen der vollständigen CFD-Lösung von der analytischen Lösung, die ja anhand der koaxialen Anordnung von Rohr und Bohrung gewonnen wurde. Die Massenströme erhält man durch Integration der axialen Geschwindigkeitsverteilung. Sowohl die durch die Spalte hindurchtretenden Massenströme als auch die dabei auftretenden Spitzengeschwindigkeiten in etwa der Mitte des Spaltes wurden im laminaren analytischen Modell bei maximaler Auslenkung etwa $20 \%$ zu hoch vorhergesagt [Dra99]. Wenn das Rohr nicht aus seiner Mittellage ausgelenkt wird, liegt der Fehler bei nur etwa $1 \%$ [Fis98b,c]. Die Tatsache, daß bei größer werdender Auslenkung sowohl der Massenstrom als auch die Spitzengeschwindigkeit bei laminarer Rechnung zu niedrig vorhergesagt werden, hängt offenbar damit zusammen, daß an der breiter werdenden Stelle die Voraussetzung der laminaren Strömung nicht mehr erfüllt ist und sich ein teilweise turbulentes Strömungsprofil ausbilden kann. Die Spalte der in den Experimenten zur vorliegenden Arbeit untersuchten Rohrbündel liegen im Gültigkeitsbereich der hergeleiteten Formel.

In [Fis99a] werden auch noch die Geschwindigkeitsverteilungen der Verdrängerströmung im Spalt infolge der Rohrbewegung bestimmt. Für die radiale Verteilung der Azimutalgeschwindigkeit erhält man

$$
w_{\varphi}(r, \varphi)=v_{R} \sin (\varphi-\beta) \frac{\left(r_{A}-r\right) \cdot\left(r_{A}^{2}+r_{A} r_{I}+4 r_{I}^{2}-3 r\left(r_{A}+r_{I}\right)\right)}{\left(r_{A}-r_{I}\right)^{3}}
$$

und für die Verteilung der Radialgeschwindigkeit

$$
w_{r}(r, \varphi)=v_{R} \cos (\varphi-\beta) \frac{\left(r_{A}-r\right) \cdot\left(r r_{A}^{3}+\left(r^{2}+r_{A} r+r_{A}^{2}\right) r_{I}^{2}-2 r r_{I}^{3}\right)}{r\left(r_{A}^{4}-2 r_{A} r_{I}^{3}-r_{I}^{4}\right)}
$$


Die genauen Herleitungen sind in [Fis99a] beschrieben. Damit ist die Geschwindigkeitsverteilung im Spalt zu jedem Zeitpunkt bekannt, da ja die Eingangsgrößen Verschiebung und Geschwindigkeit des Rohres ohne weitere Einschränkungen, also auch zeitabhängig, angegeben werden können. Explizit von der Zeit abhängige Terme treten nicht auf, da die gewählten Ansatzfunktionen von einem zu jedem Zeitpunkt bereits vollständig ausgebildetem Geschwindigkeitsprofil ausgehen. Diese Annahme erscheint aufgrund der geringen im Spalt bewegten Fluidmassen gerechtfertigt.

Die analytisch berechneten Geschwindigkeitsverteilungen können als innere Randbedingungen in die strömungsmechanische Berechnung aufgenommen werden. Allerdings ergab die Implementierung, daß Azimutal-, Axial- und Radialgeschwindigkeiten in den Spalten insbesondere auf das Querströmungsfeld im Mantelraum keinen nennenswerten Einfluß ausüben. Lediglich der Druckverlust des gesamten Wärmetauschers verringert sich in Übereinstimmung mit den Erkenntnissen aus [Fis98b,c] bei Berïcksichtigung der Axialgeschwindigkeiten in den Spalten. Zur Beurteilung der Schwingungsgefährdung ist diese Betrachtung aber nicht relevant. Schwerwiegender ist der Einfluß der sehr hohen Azimutalgeschwindigkeiten auf die Stabilität des Gesamtverfahrens. Durch die hohen lokalen Strömungsgeschwindigkeiten erhöht sich in der Nähe der Spalte die CFL-Zahl (vgl. 2.4.1, Seite 72) sehr stark. Um Stabilität zu gewährleisten, muß dann ein sehr kleiner Zeitschritt verwendet werden. Um Rechenzeit einzusparen, wurde dieser Berechnungsteil schließlich wieder aus dem Programmcode gestrichen. In Kapitel 2.2.4.3 (Seite 60) werden die vorgestellten Gleichungen (2-53) und (2-54) aber als Ausgangspunkt für die analytische Berechnung der mechanisch bedeutsamen Filmkräfte im Lager infolge der Verdrängungsströmung herangezogen. Daher ist die Vorstellung der Gleichungen (2-52) bis (2-54) an dieser Stelle im Rahmen der strömungsmechanischen Berechnungen gerechtfertigt. 


\subsection{Simulation der strukturmechanischen Rohrbündeldynamik}

\subsubsection{Strukturmechanische Grundgleichungen}

Das Strukturmodell für die Rohrschwingungen verwendet die einfache Theorie des Biegebalkens von Bernoulli. Um die Bewegungsgleichung für einen Balkenschwinger $\mathrm{zu}$ erhalten, wird an einem infinitesimalen Balkenelement das dynamische Momentengleichgewicht unter Vernachlässigung der Rotationsträgheit des Elements gebildet [Pfe92]

$$
\frac{\partial M}{\partial x}-Q+F \frac{\partial w}{\partial x}=0
$$

Für das Biegemoment $M$ gilt aus der Elastostatik der Zusammenhang [Pfe92]

$$
M=-E I \frac{\partial^{2} w}{\partial x^{2}}
$$

Aus dem dynamischen Kräftegleichgewicht

$$
m \frac{\partial^{2} w}{\partial t^{2}}=\frac{\partial Q}{\partial x}-\zeta \frac{\partial w}{\partial t}+q
$$

zwischen Trägheitskräften, Querkräften, geschwindigkeitsabhängigen Dämpfungskräften und äußerer Streckenlast am infinitesimalen Element ergibt sich bei konstanten Querschnittsgrößen die Bew egungsgleichung

$$
m \frac{\partial^{2} w}{\partial t^{2}}+\zeta \frac{\partial w}{\partial t}+E I \frac{\partial^{4} w}{\partial x^{4}}-F \frac{\partial^{2} w}{\partial x^{2}}-\frac{\partial F}{\partial x} \frac{\partial w}{\partial x}=q
$$

Die äußere Streckenquerlast $q$ resultiert aus der Umströmung der Wärmetauscherrohre sowie zusätzlichen Kräften aus den Reibstößen und Strömungsvorgängen in den Spalten zwischen den Leitblechen und den Rohren.

Die innere Dämpfung $\zeta$ der Rohre kann grundsätzlich über Ausschwingversuche experimentell bestimmt werden. Hierzu muß das logarithmische Dekrement

$$
\Lambda=\ln \left|\frac{w^{n}}{w^{n+1}}\right|=\ln \left|\frac{\ddot{w}^{n}}{\ddot{w}^{n+1}}\right|
$$

aus Amplituden- oder Beschleunigungsmessungen zweier aufeinanderfolgender Extrema ermittelt werden. Aus dem zeitlichen Verlauf der gemessenen Reihe kann dann 


$$
\zeta=\frac{4 m \Lambda}{\Delta T}
$$

berechnet werden, wobei $\Delta T$ der zeitliche Abstand der beiden Extrema ist. Die innere Dämpfung ist jedoch im vorliegenden Problemkreis nur wenig bedeutsam, da die von der Mantelraumströmung verursachten Dämpfungskräfte um Größenordnungen höher ausfallen. Die gemischte Fluid-Struktur-Dämpfung kann aber auf die gleiche Weise bestimmt werden, etwa zum Zwecke der Anwendung in halbempirischen Stabilitätskriterien.

Die Axialkraft $F$ ergibt sich aus Fluidlasten in Längsrichtung, Lagerkräften in Längsrichtung, dem Eigengewicht der Rohre in Längsrichtung, Kräften in Längsrichtung aus Reibstößen in den Umlenkblechen, Kräften aus vorhandenem Innendruck im Behälter, Temperaturspannungen oder evtl. vorhandenen Montagevorspannungen. Außer den Montagevorspannungen haben diese Kräfte jedoch in der zu betrachtenden Versuchseinrichtung keinen entscheidenden Einfluß auf das Schwingverhalten, ebenso wie die nur geringen Torsionsspannungen. Daher bleibt die Längskraft über der Rohrlänge konstant und es muß für das infinitesimale Element kein dynamisches Kräftegleichgewicht in Rohrlängsachsenrichtung gelöst werden, was eine bedeutende numerische Vereinfachung darstellt. Die zu betrachtende Bewegungsgleichung für ein gedämpftes, vorgespanntes Rohr lautet damit

$$
m \frac{\partial^{2} w}{\partial t^{2}}+\zeta \frac{\partial w}{\partial t}+E I \frac{\partial^{4} w}{\partial x^{4}}-F \frac{\partial^{2} w}{\partial x^{2}}=q
$$

Die erhaltene Schwingungsgleichung gilt für die $y$-Richtung ebenso wie für die $z$-Richtung. Im Trägheitsterm $m$ muß außer der Masse des Rohrwerkstoffs noch die Masse des im Rohrinneren mitbewegten Fluids berücksichtigt werden [Che76, VDI97]. Für analytische Lösungen ist auch die hydrodynamische Zusatzmasse des verdrängten Mantelraumfluids zu berücksichtigen, während bei numerischer Simulation diese zusätzlichen Trägheitskräfte aus den Druck- und Scherkräften an den Rohroberflächen infolge der Mantelraumströmung resultieren und damit bereits implizit in der Längsstreckenlast $q$ enthalten sind.

\subsubsection{Räumliche Diskretisierung}

Numerische strukturdynamische Berechnungen werden heutzutage wegen ihrer flexiblen Einsatzmöglichkeiten normalerweise mit Finite-Elemente- 
Programmen durchgeführt [Bat86, Zie84]. Im vorliegenden Fall ist jedoch die Formulierung der Diskretisierung der Bewegungsgleichung (2-61) für das zu entwickelnde Programm mit Hilfe von Finiten Differenzen [Smi70] deutlich einfacher durchzuführen. Um die Rohrbewegung numerisch bestimmen zu können, wird das Rohr in $n_{x}+1$ äquidistante Abschnitte eingeteilt. In Abbildung 28 ist die Diskretisierung nochmals verdeutlicht.

Die Ableitungen nach dem Ort werden durch finite Differenzenquotienten zweiter Ordnung ersetzt. Für eine beliebige Stelle $I$ erhält man dadurch in impliziter Formulierung zum Zeitpunkt $n+1$ die Gleichung

$$
\begin{gathered}
m \ddot{w}_{I}^{n+1}+\zeta \dot{w}_{I}^{n+1}-\frac{F}{\Delta x^{2}}\left(w_{I-1}^{n+1}-2 w_{I}^{n+1}+w_{I+1}^{n+1}\right)+ \\
+\frac{E I}{\Delta x^{4}}\left(w_{I-2}^{n+1}-4 w_{I-1}^{n+1}+6 w_{I}^{n+1}-4 w_{I+1}^{n+1}+w_{I+2}^{n+1}\right)=q_{I}^{n+1}
\end{gathered}
$$

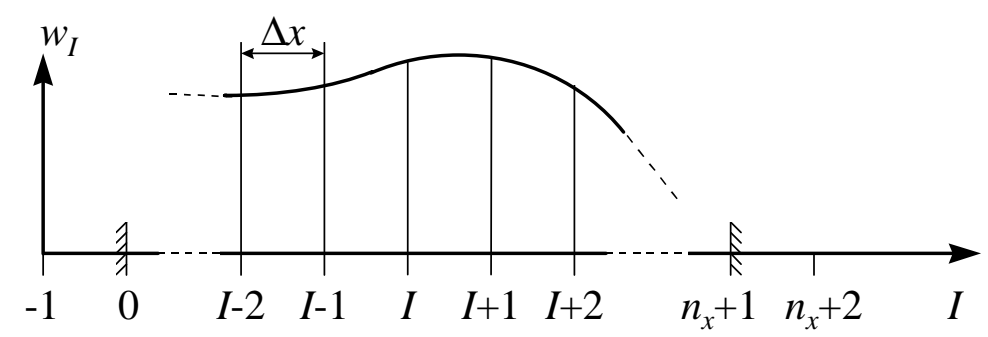

Abbildung 2-8: Diskretisierung der Rohrbewegungsgleichung.

In den Lagern an den Einspannrändern gelten die Randbedingungen

$$
w_{0}^{n+1}=0, \quad w_{n_{x}+1}^{n+1}=0
$$

sowie z.B. bei $I=0$ für gelenkige Lagerung

$$
w_{0}^{\prime \prime n+1}=0 \Rightarrow w_{-1}^{n+1}-2 w_{0}^{n+1}+w_{1}^{n+1}=0
$$

bzw. für feste Einspannung

$$
w_{0}^{\prime n+1}=0 \quad \Rightarrow \quad w_{1}^{n+1}-w_{-1}^{n+1}=0
$$

Durch Subtraktion der Randbedingungen (2-64) bzw. (2-65) von (2-62) und Einsetzen von (2-63a) für $I=1$ bzw. (2-63b) für $I=n_{x}$ ergibt sich die symmetrische Pentadiagonal-Steifigkeitsmatrix 


$$
\mathbf{K}=\frac{E I}{\Delta x^{4}}\left(\begin{array}{ccccccccc}
\lambda_{L} & -4 & 1 & 0 & \cdots & \cdots & \cdots & \cdots & 0 \\
-4 & 6 & -4 & 1 & 0 & & & & \vdots \\
1 & -4 & 6 & -4 & 1 & 0 & & & \vdots \\
0 & 1 & -4 & 6 & -4 & 1 & 0 & & \vdots \\
\vdots & \ddots & \ddots & \ddots & \ddots & \ddots & \ddots & \ddots & \vdots \\
\vdots & & 0 & 1 & -4 & 6 & -4 & 1 & 0 \\
\vdots & & & 0 & 1 & -4 & 6 & -4 & 1 \\
\vdots & & & & 0 & 1 & -4 & 6 & -4 \\
0 & \cdots & \cdots & \cdots & \cdots & 0 & 1 & -4 & \lambda_{R}
\end{array}\right)+\frac{F}{\Delta x^{2}}\left(\begin{array}{ccccccc}
2 & -1 & 0 & \cdots & \cdots & \cdots & 0 \\
-1 & 2 & -1 & \ddots & & & \vdots \\
0 & -1 & 2 & -1 & \ddots & & \vdots \\
\vdots & \ddots & \ddots & \ddots & \ddots & \ddots & \vdots \\
\vdots & & \ddots & -1 & 2 & -1 & 0 \\
\vdots & & & \ddots & -1 & 2 & -1 \\
0 & \cdots & \cdots & \cdots & 0 & -1 & 2
\end{array}\right)
$$

mit den Lagerfaktoren $\lambda$, die für gelenkige Lagerung den Wert 5 und für steife Lagerung den Wert 7 annehmen. Werte zwischen 5 und 7 bedeuten dann weiche Lagerungen, die von ihrer Charakteristik her zwischen den beiden Extremfällen liegen. Für eine symmetrische Lagerung kann der Lagerfaktor $\lambda$ prinzipiell experimentell über die Messung der Eigenfrequenzen oder über den Vergleich mit analytischen Lösungen für weiche Einspannungen bestimmt werden. Damit kann auf einfache Weise eine korrekte Simulation von Lagersteifigkeiten erfolgen.

Abbildung 2-9 zeigt für einen Beispielfall die Abhängigkeit der simulierten ersten bis dritten Eigenfrequenz vom Lagerfaktor. Da die Kurven zum Wert 7 hin sehr flach verlaufen, wird für viele Anwendungen, wie auch in der vorliegenden Arbeit aufgrund der mit einer Wandstärke von $50 \mathrm{~mm}$ sehr steif ausgeführten Rohrplatte, die Annahme $\lambda=7$ völlig ausreichend sein.

Mit der symmetrischen Diagonal-Massenmatrix

$$
\mathbf{M}=\left(\begin{array}{cccc}
m & 0 & \cdots & 0 \\
0 & \ddots & \ddots & \vdots \\
\vdots & \ddots & \ddots & 0 \\
0 & \cdots & 0 & m
\end{array}\right)
$$

und der symmetrischen Diagonal-Dämpfungsmatrix

$$
\mathbf{C}=\left(\begin{array}{cccc}
\zeta & 0 & \cdots & 0 \\
0 & \ddots & \ddots & \vdots \\
\vdots & \ddots & \ddots & 0 \\
0 & \cdots & 0 & \zeta
\end{array}\right)
$$


erhält man schließlich für den Zeitpunkt $n+1$ die diskretisierte Bewegungsgleichung

$$
\mathbf{M} \ddot{\bar{w}}^{n+1}+\mathbf{C} \dot{\bar{w}}^{n+1}+\mathbf{K} \vec{w}^{n+1}=\vec{q}^{n+1}
$$

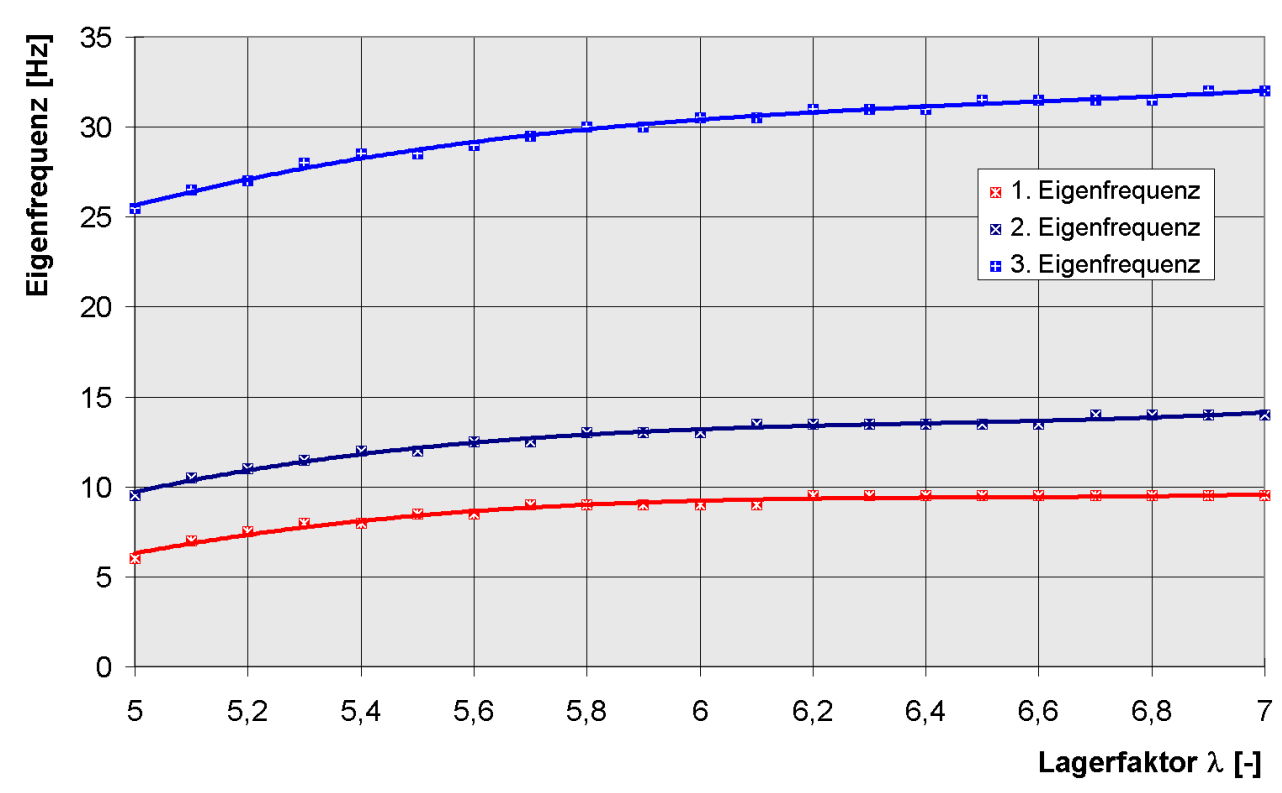

Abbildung 2-9: Abhängigkeit der numerisch simulierten Rohreigenfrequenzen von den Lagerfaktoren.

\subsubsection{Zeitliche Diskretisierung}

Zur zeitlichen Diskretisierung und Integration wurde vorgeschlagen, auch Differenzenquotienten für die zeitlichen Ableitungen einzuführen und gemäß dem Verfahren von Crank und Nicholson die vierte Ortsableitung zwischen dem bekannten und dem neuen Zeitschritt zu wichten, um das Verfahren zu stabilisieren [Fis95b]. Allerdings entsteht durch dieses Verfahren numerische Dämpfung, die die Amplitude einer einmal angestoßenen Schwingbewegung mit der Zeit ohne physikalischen Grund immer weiter abnehmen läßt [Bat86]. Zudem ist dieser Fehler von der Zeitschrittweite abhängig [Bat86]. Auch die in [Bat86] beschriebenen Verfahren nach Wilson und Houboldt führen zu numerischer Diffusion. Daher wird zur zeitlichen Integration das Verfahren nach Newmark [New59, Bat86, Knu79] verwendet, das von numerischer Dämpfung frei ist. Zudem ist das Verfahren nach Newmark mit dem kleinsten Fehler in der Bestimmung der Periodenlänge behaftet [Bat86]. Zunächst wird für Ort und Geschwindigkeit zum Zeitpunkt $n+1$ 


$$
\vec{w}^{n+1}=\vec{w}^{n}+\dot{\bar{w}}^{n} \Delta t+\left[\left(\frac{1}{2}-\alpha\right) \ddot{\vec{w}}^{n}+\alpha \ddot{\bar{w}}^{n+1}\right] \Delta t^{2}
$$

und

$$
\dot{\bar{w}}^{n+1}=\dot{\bar{w}}^{n}+\left[(1-\delta) \ddot{\ddot{w}}^{n}+\delta \ddot{\ddot{w}}^{n+1}\right] \Delta t
$$

gesetzt. (2-70) wird nach $\ddot{\ddot{w}}^{n+1}$ aufgelöst. $\ddot{\bar{w}}^{n+1}$ wird dann in die Bewegungsgleichung (2-69) und in die Geschwindigkeitsdefinition (2-71) eingesetzt. Dann wird noch die neue Geschwindigkeit $\dot{\vec{w}}^{n+1}$ in (2-69) eingesetzt. Newmark weist in [New59] nach, daß für $\delta=1 / 2$ die numerische Diffusion verschwindet. Außerdem zeigt er, daß das Verfahren für $\alpha=1 / 4$ für alle Zeitschritte stabil ist. Man spricht dann vom Verfahren der konstanten mittleren Beschleunigung. Bei diesem Verfahren ist der Fehler in der Energiebilanz Null [Knu79]. Der Zeitschritt zum Erreichen einer konvergenten Lösung darf höchstens das 0,318-fache der kürzesten vorkommenden Periodendauer betragen [New59]. Dieser Wert wird jedoch praktisch immer zu unterschreiten sein. Setzt man daher die Zahlenwerte für $\alpha$ und $\delta$ noch in (2-69) ein, erhält man durch Ordnen für $\vec{w}^{n+1}$ das lineare Gleichungssystem

$$
\begin{gathered}
\left(4 \mathbf{M}+2 \Delta t \mathbf{C}+\Delta t^{2} \mathbf{K}\right) \vec{w}^{n+1}=\Delta t^{2} \vec{q}^{n+1}+ \\
+(4 \mathbf{M}+2 \Delta t \mathbf{C}) \vec{w}^{n}+\left(4 \Delta t \mathbf{M}+\Delta t^{2} \mathbf{C}\right) \dot{\bar{w}}^{n}+\Delta t^{2} \mathbf{M} \ddot{\bar{w}}^{n}
\end{gathered}
$$

Die Größen der rechten Seite sind aus dem vorangegangenen Zeitschritt oder im Falle der Streckenlast als iterativ verbesserter neuer Startwert aus dem Strömungsmodul bekannt. Die neuen Geschwindigkeiten und Beschleunigungen werden jeweils aus (2-70) und (2-71) bestimmt. Ein weiterer Vorteil des Newmark-Verfahrens ist, daß von Zeitschritt zu Zeitschritt unterschiedliche Zeitschrittweiten verwendet werden können. Dies ermöglicht grundsätzlich eine konvergenzabhängige automatische Zeitschrittweitensteu erung.

\subsubsection{Zusätzliche Randbedingungen an den Leitblechen}

Die Bohrungen in den Leitblechen müssen aus Montagegründen mit einem gewissen Übermaß ausgestattet sein. Nach DIN 28182 werden z.B. diametrale Montageübermaße von 0,7 mm für Rohre mit bis zu $1000 \mathrm{~mm}$ freier Stützweite empfohlen [DIN80]. Dadurch ergibt sich in montiertem Zustand idealisiert ein Ringspalt, dessen Radialmaß jedoch im Vergleich zu den Durchmessern von Rohr bzw. Bohrung klein ist. 
Bedeutsam sind die strukturmechanischen Auswirkungen der Reibstöße. Zudem entstehen im Spalt selber bei dynamischer Bewegung der Rohre Zusatzkräfte aufgrund von Fluiddruck und Fluidreibung, die die Bewegung des Rohrs tendenziell hemmen wollen und so eine zusätzliche Dämpfung am bewegten Rohr hervorrufen [Mul80, Zho96]. Der Spalt führt außerdem dazu, daß die einzelnen Rohre nicht zu jedem Zeitpunkt an jedem Lager gestützt sind [Rog76]. Dadurch werden einerseits zeitweise niedrigere Rohreigenfrequenzen wirksam, andererseits werden durch die Reibstöße auch höhere Eigenfrequenzen angeregt.

\subsubsection{Modifikation der Steifigkeitsmatrix an der Lage rstelle}

Tritt ein Rohr mit der Innenseite einer Leitblechbohrung in Kontakt, so wirkt die Stützstelle wie ein Spitzenlager, in dem kein Biegemoment aufgenommen wird. Links und rechts vom Spitzenlager nähert sich die Biegelinie asymptotisch ihrer Tangente und ihrem Krümmungskreis im Lagerpunkt an. Daher sind die ersten und zweiten Ableitungen der Biegelinie links und rechts vom Lagerpunkt jeweils gleich. In diskretisierter Formulierung für die Punkte links und rechts vom Lagerpunkt $s$ gilt daher

$$
\begin{gathered}
w_{L}^{\prime}=\frac{w_{s}^{n+1}-w_{s-1}^{n+1}}{\Delta x}=\frac{w_{s+1}^{n+1}-w_{s}^{n+1}}{\Delta x}=w_{R}^{\prime} \\
w_{L}^{\prime \prime}=\frac{w_{s-2}^{n+1}-2 w_{s-1}^{n+1}+w_{s}^{n+1}}{\Delta x^{2}}=\frac{w_{s+2}^{n+1}-2 w_{s+1}^{n+1}+w_{s}^{n+1}}{\Delta x^{2}}=w_{R}^{\prime \prime}
\end{gathered}
$$

Vereinfacht ergeben sich die zwei Bedingungen

$$
\begin{array}{r}
w_{s-1}^{n+1}-2 w_{s}^{n+1}+w_{s+1}^{n+1}=0 \\
w_{s-2}^{n+1}-2 w_{s-1}^{n+1}+2 w_{s+1}^{n+1}-w_{s+2}^{n+1}=0
\end{array}
$$

Um die Randbedingungen in die Lösungsmatrix einzubringen, wird zunächst (2-74a) von der Bewegungsgleichung (2-62) bei $I=s+1$ und $I=s-1$ subtrahiert. (2-74b) wird zur Bewegungsgleichung (2-62) bei $I=s+1$ hinzuaddiert und von der Bewegungsgleichung (2-62) bei $I=s-1$ subtrahiert, und man erhält im längskraftunabhängigen Teil der Steifigkeitsmatrix modifizierte Zeilenvektoren der Form

$$
\begin{aligned}
\vec{K}_{s-1}^{T} & =\left(\begin{array}{llllllllllll}
0 & \cdots & 0 & 1 & -5 & 9 & -6 & 0 & 1 & 0 & \cdots & 0
\end{array}\right) \\
\vec{K}_{s+1}^{T} & =\left(\begin{array}{llllllllllll}
0 & \cdots & 0 & 1 & 0 & -6 & 9 & -5 & 1 & 0 & \cdots & 0
\end{array}\right)
\end{aligned}
$$


Außerdem vermindert sich die Ordnung des Systems um Eins, da die Durchsenkung $w_{s}^{n+1}$ am Lagerpunkt bekannt ist. Die dargestellte Modifikation der Steifigkeitsmatrix muß sowohl für gelenkige Lager ohne Spalt als auch für Lager mit Kontakt angewendet werden.

\subsubsection{Mechanische Zusatzkräfte infolge der Reibstöße im Leitblech}

Reale Zwischenlager haben nicht nur die Eigenschaften einer gelenkigen Spitzenlagerung, sondern die Rohre können auch im Rahmen des Lagerspiels zunächst frei schwingen und verformen sich dann beim Auftreffen auf die Wandung der Leitblechbohrung elastisch [Zho96, Rog76, Che85]. Dadurch wirkt eine zusätzliche äußere Lagerkraft auf das Rohr ein. Es treten zwei Nichtlinearitäten auf: Zum einen die Abfrage des Kontaktproblems selbst, zum anderen können die Lagerstellen im allgemeinen Fall nichtlineare Federkennlinien haben. Im vorliegenden Fall wurde ein an die Arbeiten von Hertz [Her82] angelehntes Gesetz für die Normalkraft zwischen zwei aufeinander treffenden Körpern verwendet,

$$
F_{n}=k_{n} \Delta n^{\alpha}+\zeta_{n} \dot{w}_{n},
$$

das aus numerischen Gründen noch mit einem kleinen Dämpfungsterm [Ant90] versehen wurde. Diese zusätzliche Lagerkraft muß auf die Lagerbreite bezogen werden und dann in den numerischen Gleichungen zur Streckenlast im Lagerpunkt hinzuaddiert werden.

Um reale Lagerverhältnisse exakt simulieren zu können, müssen die Kontaktsteifigkeit und der Exponent in (2-76) im statischen Experiment oder in einer numerischen Rechnung bestimmt werden. Oft wird für den Exponenten $\alpha$ der Wert 1,0 verwendet, und die Steifigkeit $k_{n}$ kann analytisch gemäß [Axi88]

$$
k_{n}=1,9 \frac{E e^{2}}{D} \sqrt{\frac{e}{D}}
$$

berechnet werden. Hierbei wird allerdings offenbar davon ausgegangen, daß es sich um eine Punktlast [Luk79] handelt. In [Luk79] wird auch der idealisierte Vorfaktor mit 1,919 etwas genauer angegeben.

Punktlasten werden sich zwischen Rohr und Leitblech nur dann ergeben, wenn lokal ein gewisser Verkippungswinkel $\beta_{V}$ zwischen Rohrlängsachse und Bohrungsachse besteht. Liegt das Rohr jedoch entlang einer Linie in der Leitblechbohrung an, so muß die Steifigkeit des Kontaktes ansteigen, da die wirkende 
Kraft entlang der Linie verteilt aus Energiegründen nur noch eine kleinere Ovalisierung des Rohrs bewirken kann. Deininger und Fischer [Dei01] haben numerische und experimentelle Bestimmungen der Kontaktsteifigkeiten vorgenommen. Für die numerischen Lösungen (FE-Lösung) wurde das Finite-ElementeProgramm MARC/Mentat verwendet. Danach nähern sich die Kontaktsteifigkeiten mit zunehmendem Verkippungswinkel $\beta_{V}$ vom Idealfall des entlang einer Linie anliegenden Rohrs einer Exponentialfunktion folgend dem Idealfall der Punktberührung gemäß Gleichung (2-77) an. Für den vorliegenden Fall konnte für die Kontaktsteifigkeit die Regressionsgleichung

$$
k_{n}=k_{F E} \cdot\left[1-A\left(1-\exp \left(-B \beta_{V}\right)\right)\right]
$$

gefunden werden, wobei $k_{F E}$ die winkelabhängige Kontaktsteifigkeit gemäß FELösung ist. Für größer werdende Winkel $\beta_{V}$ nähert sich Gleichung (2-78) der theoretischen Lösung der Punktberührung nach Gleichung (2-77) an. Die Kontaktsteifigkeit $k_{F E}$ steigt außerdem bei Linienberührung von der theoretischen Lösung für Punktberührung bei verschwindender Leitblechdicke gemäß Gleichung (2-77) linear mit der Leitblechdicke an. In Tabelle 2-1 sind die sich aus den FE-Berechnungen in [Dei01] ergebenden Anpassungsfaktoren $A$ und $B$ für die verwendeten Aluminiumrohre $12 \mathrm{~mm}$ und $16 \mathrm{~mm}$ angegeben. Der Verkippungswinkel $\beta_{V}$ in Winkelgrad ergibt sich in der Simulation aus den Steigungen der Biegelinien in $y$ - und $z$-Richtung. Die FE-Simulationen in [Dei01] ergaben linearelastisches Verhalten auch bei Punktberührung, so daß der Wert 1,0 für den Exponenten $\alpha$ in Gleichung (2-76) jedenfalls gerechtfertigt ist. Axisa et al. [Axi88] vertreten die Auffassung, daß eine hohe Genauigkeit für $k_{n}$ nicht immer erforderlich ist, für den vorliegenden Fall einer genauen Simulation muß dies im Allgemeinen bezweifelt werden. Jedoch ergeben sich ausgehend von dem Fall der Linienberührung ( $\beta_{V}=0 \mathrm{Grad}$ ) schon bei kleinen Verkippungswinkeln starke Reduzierungen der Kontaktsteifigkeiten, so daß bei im Mittel größeren auftretenden Verkippungswinkeln auch die Anwendung von Gleichung (2-77) gerechtfertigt ist.

Die tangential aufgrund von Reibung auftretenden Kräfte wurden unter Berücksichtigung einer Unterscheidung zwischen Haftreibung und Gleitreibung modelliert. Für die Gleitreibung gilt das Gesetz von Coulomb [Böh87, Dre59]

$$
F_{t}=\mu_{t} F_{n} .
$$




\begin{tabular}{|c|c|c|}
\hline Rohrdurchmesser & $A$ & $B$ \\
\hline $12 \mathrm{~mm}$ & 0,373764 & 8,915525 \\
\hline $16 \mathrm{~mm}$ & 0,224902 & 9,184227 \\
\hline
\end{tabular}

Tabelle 2-1: Konstanten des Kontaktsteifigkeitsmodells nach Gleichung (2-78).

Der Gleitreibungkoeffizient ist von der Tangentialgeschwindigkeit abhängig. In [Rog76] steigt der Gleitreibungskoeffizient bis zu einer Grenzgeschwindigkeit linear an, um dann konstant zu bleiben. Mit Hilfe einer tanh-Funktion kann der Übergang zwischen beiden Bereichen geglättet werden. In der vorliegenden Arbeit wurde daher der Zusammenhang

$$
\mu_{t}=\mu_{G l} \tanh \frac{\dot{w}_{t}}{\dot{\varepsilon}_{t}}
$$

verwendet. Wenn

$$
F_{t}<\mu_{0} F_{n}
$$

gilt, dann herrscht Haftreibung und die Haftreibungkräfte werden durch ein Oberflächendeformationsgesetz [Ode83]

$$
F_{t}=F_{n}\left(\mu_{t} \tanh \frac{\varepsilon}{\varepsilon_{0}}+\zeta_{t} \dot{w}_{t}\right)
$$

modelliert, wobei wiederum aus numerischen Gründen ein kleiner Dämpfungsterm hinzugefügt wurde.

Das Newmark-Verfahren [New59] hat entscheidenden Anteil daran, daß die Simulationen unter Einbeziehung des Kontaktproblems zwischen Leitblechen und Rohren äußerst stabil bleiben. Über ähnliche Erfahrungen berichtet Johansson [Joh97]. Auch er verwendet bei seiner Simulation eines schwingenden Zylinders das Newmark-Verfahren zur Zeitintegration und erhält bei Kontakt im Stützlager ebenfalls gute Stabilität. Er unterscheidet dabei ebenfalls zwischen Gleiten und Haften.

Für die Anregung höherer Schwingungsmoden durch das Anschlagen der Rohre im Leitblech sind die Reibungsvorgänge zunächst von sekundärer Wichtigkeit, hier überwiegt der Einfluß der Kontaktsteifigkeiten. Die genaue Betrachtung von Reibungsvorgängen wird aber dann bedeutsam, wenn Verschleißvorgänge (fretting wear) zwischen Rohren und Leitblechen au sgewertet werden sollen. 


\subsubsection{Filmkräfte aufgrund von Spaltströmung}

Zur Bestimmung der Druckkräfte auf das Rohr infolge der im Spalt entstehenden Filmströmung muß zunächst der Druckgradient auf der Rohroberfläche in Richtung des Azimutalwinkels bestimmt werden. Für die vorliegende Arbeit wurden die zusätzlichen Kräfte analytisch in [Fis99a] unter Zuhilfenahme von Mathematica 3.0.1 [Wo197, Fis00b] hergeleitet. Der Druckgradient wird aus dem azimutalen Impulsgleichgewicht berechnet. Die Navier-Stokes-Gleichung für das azimutale Kräftegleichgewicht lautet [Tru68]

$$
\begin{gathered}
\rho \frac{\partial w_{\varphi}}{\partial t}+w_{r} \frac{\partial w_{\varphi}}{\partial r}+\frac{w_{\varphi}}{r} \frac{\partial w_{\varphi}}{\partial \varphi}+w_{x} \frac{\partial w_{\varphi}}{\partial x}+\frac{w_{r} w_{\varphi}}{r}= \\
=-\frac{1}{\rho r} \frac{\partial p}{\partial \varphi}+\frac{\eta}{\rho}\left[\frac{1}{r} \frac{\partial}{\partial r}\left(r \frac{\partial w_{\varphi}}{\partial r}\right)+\frac{1}{r^{2}} \frac{\partial^{2} w_{\varphi}}{\partial \varphi^{2}}+\frac{\partial^{2} w_{\varphi}}{\partial x^{2}}-\frac{w_{\varphi}}{r^{2}}+\frac{2}{r^{2}} \frac{\partial w_{r}}{\partial \varphi}\right]
\end{gathered}
$$

Hierbei können im Rahmen der Modellbildung die Ableitungen nach der Axialkoordinate $x$ fortgelassen werden. Durch Einsetzen der bekannten Geschwindigkeitsverläufe aus (2-53) und (2-54), des inneren Radius als Radialkoordinate und nach umfangreichen Vereinfachungen mit Hilfe von Mathematica folgt für den Druckgradienten in azimutaler Richtung

$$
\begin{aligned}
\left.\frac{\partial p}{\partial \varphi}\right|_{r=r_{I}}= & 4 \eta v_{R} \sin (\beta-\varphi) \frac{-r_{A}^{2}+2 r_{A} r_{I}+2 r_{I}^{2}}{\left(r_{A}-r_{I}\right)^{3}}+ \\
& +\rho v_{R}^{2} \sin (2(\beta-\varphi)) \frac{r_{I}\left(r_{I}+2 r_{A}\right)}{\left(r_{A}-r_{I}\right)^{2}}-\left.\rho r_{I} \frac{\partial w_{\varphi}}{\partial t}\right|_{r=r_{I}}
\end{aligned}
$$

Es verbleiben keine Ableitungen des äußeren Radius $r_{A}$ nach dem Azimutalwinkel, wodurch sich eine recht einfach aufgebaute Formel ergibt. Die analytische Integration des Druckgradienten ist aufgrund der Abhängigkeiten des äußeren Radius vom Azimutalwinkel nicht mehr möglich. Die numerische Integration führt zu Fehlern, da bei den gängigen Verfahren der Druck bei $\varphi=0$ nicht mit dem Druck bei $\varphi=2 \pi$ übereinstimmt. Daher wird das in [Fis99a] beschriebene numerische Verfahren verwendet, das auf der Anwendung finiter Differenzen und der Methode der kleinsten Fehlerquadrate beruht. Trotz der zum Schluß notwendigen numerischen Integrationen ergibt sich ein deutlicher Geschwindigkeitsvorteil gegenüber einer CFD-Berechnung der Spaltströmung. 
Als Überprüfung wurden die sich aufgrund der Bewegung analytisch aus (2-84) ergebenden Kräfte auf das Rohr mit einer numerischen Berechnung mit CFX 4.1 [AEA97, Dra99] verglichen. In Tabelle 2-2 werden beispielsweise die Kräfte auf ein Rohr mit 16 mm Durchmesser in einer Bohrung mit 16,7 mm Durchmesser bei einer mittleren Auslenkung von $0,175 \mathrm{~mm}$ in $y$-Richtung und fünf in unterschiedliche Richtungen wirkende Rohrgeschwindigkeiten von 0,1 $\mathrm{m} / \mathrm{s}$ aus analytischen und numerischen Berechnungen einander gegenübergestellt.

\begin{tabular}{|c|c|c|c|c|c|c|}
\hline & $\gamma[\mathrm{Grad}]$ & $0^{0}$ & $45^{0}$ & $90^{0}$ & $135^{0}$ & $180^{0}$ \\
\hline \multirow{3}{*}{ Analytisch } & $F_{y}[\mathrm{~N}]$ & $-6,06$ & $-4,98$ & $-2,55$ & $-0,46$ & 0,01 \\
\cline { 2 - 7 } & $F_{z}[\mathrm{~N}]$ & 0,00 & $-2,26$ & $-2,91$ & $-1,61$ & 0,60 \\
\hline \multirow{3}{*}{ Numerisch } & $F_{y}[\mathrm{~N}]$ & $-4,37$ & $-1,76$ & $-0,08$ & 0,44 & 1,41 \\
\cline { 2 - 7 } & $F_{z}[\mathrm{~N}]$ & 0,00 & $-0,40$ & $-1,98$ & $-1,03$ & 0,00 \\
\hline
\end{tabular}

Tabelle 2-2: Vergleich der Spaltkräfte auf ein bewegtes Rohr bei analytischer und numerischer Rechnung.

Der Vergleich zwischen numerischen und analytischen Ergebnissen zeigt hinsichtlich der radialen Kräfte auf das Rohr infolge des Gleitfilms im Spalt zumindest eine größenordnungsmäßige Übereinstimmung. Der Vergleich der Druck- und Reibungskräfte zeigt, daß der Anteil der Reibungskraft an der Gesamtkraft auf das Rohr zu vernachlässigen ist, nicht jedoch der Einfluß der Viskosität auf den azimutalen Druckgradienten gemäß (2-84). Die zusätzlichen Filmkräfte stimmen größenordnungsmäßig mit Simulationsergebnissen überein. In [Zho96] wird ebenfalls ein Modell zur Berechnung der Spaltströmungskräfte vorgestellt. Es basiert jedoch maßgeblich auf den Kräften infolge der Axialströmung im Leitblech, unterscheidet sich also grundsätzlich vom Berechnungsansatz im Vergleich zu Gleichung (2-84). Obwohl der Berechnungsgang unterschiedlich ist, erhalten auch Zhou und Rogers in [Zho96] Bestimmungsgleichungen für die Spaltkräfte, die einen staudruckabhängigen Term, einen Reibungs-Geschwindigkeits-Term und einen beschleunigungsabhängigen Term enthalten. Für die Berücksichtigung der Leckageeffekte, die die Reibungskraft verringern, werden Korrekturfaktoren der Form 


$$
L_{i}=1-\tanh \left(\lambda_{i} s / D\right)
$$

verwendet mit $\lambda_{i}$ als Eigenwerten der Leckagefaktoren, die sich aus der Position des Rohrs im Leitblech ergeben [Zho96]. Zur Anpassung von Gleichung (2-84) werden diese Leckagefaktoren in die Bestimmung des Druckgradienten mit eingebracht. Die in den Simulationen verwendete Gleichung für den azimutalen Druckgradienten wird damit

$$
\begin{aligned}
\left.\frac{\partial p}{\partial \varphi}\right|_{r=r_{I}}= & 4 \eta v_{R} \sin (\beta-\varphi) \frac{-r_{A}^{2}+2 r_{A} r_{I}+2 r_{I}^{2}}{\left(r_{A}-r_{I}\right)^{3}} L_{1}+ \\
& +\rho v_{R}^{2} \sin (2(\beta-\varphi)) \frac{r_{I}\left(r_{I}+2 r_{A}\right)}{\left(r_{A}-r_{I}\right)^{2}} L_{3}-\left.\rho r_{I} \frac{\partial w_{\varphi}}{\partial t}\right|_{r=r_{I}} L_{2}
\end{aligned}
$$

\subsubsection{Zusammenfassung und Beispielrechnung Kontaktmodell}

Durch die Einführung des Kontaktmodells konnte das deterministischchaotische Schwingungsverhalten der Rohre im Rohrbündel nachgerechnet werden. Abbildung 2-10 zeigt ein Beispiel für eine Trajektorie eines Rohrmittelpunktes im sog. „center circle“, der die Bahn des Rohrmittelpunktes beim Abrollen des Rohrs auf der Bohrungsinnenseite darstellt.

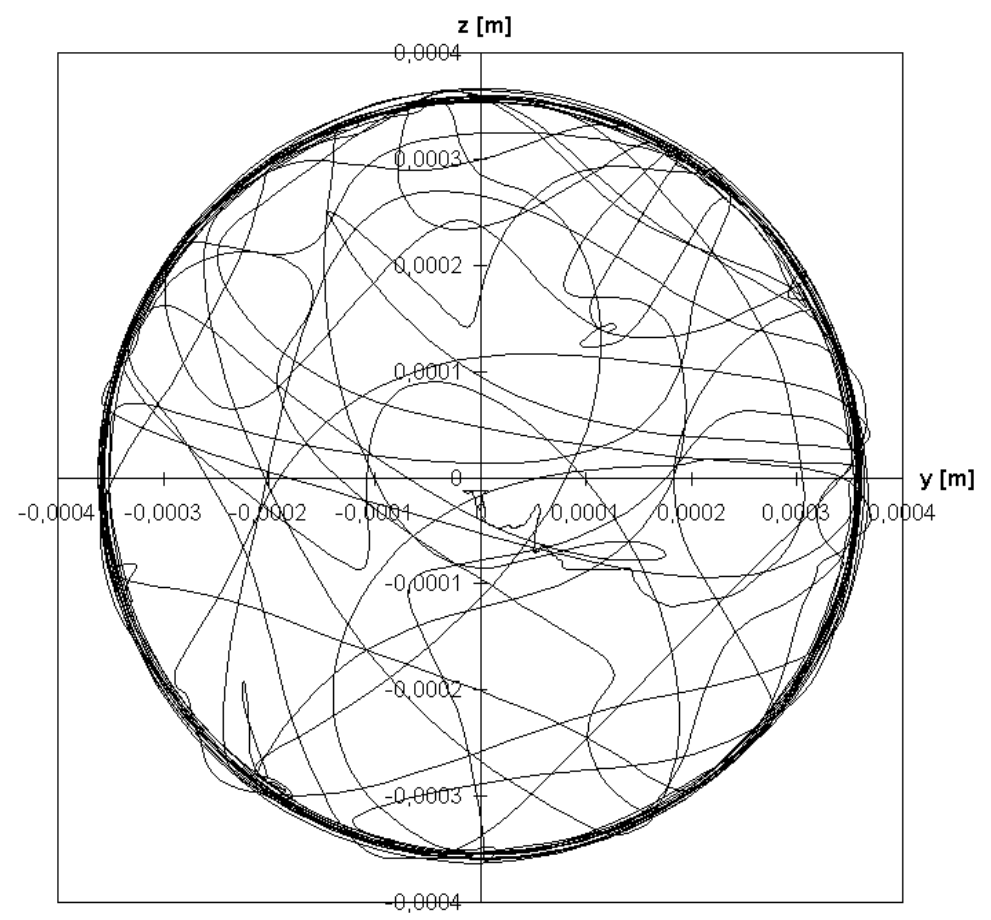

Abbildung 2-10: Trajektorie des Rohrmittelpunktes innerhalb eines ,center circles“. 
Das Anschlagen der Rohre untereinander wurde nicht deshalb berücksichtigt, weil es etwa einen sinnvollen Betriebsfall für Wärmetauscher darstellen würde. Vielmehr wurde diese als „Katastrophenfall“ für den Betrieb anzusehende Situation mit abgedeckt, um in extremen Situationen das Programm nicht zum Absturz zu bringen, wodurch wertvolle Rechenzeit verschwendet würde.

Denkbar wäre z.B., daß die Rampenzeit zum Hochlaufen auf den Betriebsmassenstrom vom Anwender zu kurz gewählt wurde. Dadurch würden im Hochlaufvorgang hohe instationäre Kräfte auftreten, die kurzzeitig wesentlich stärkere Rohrdeformationen und damit evtl. ein Anschlagen der Rohre untereinander hervorrufen würden. Denkbar wäre weiter, daß außerdem ein Endmassenstrom gewählt wurde, bei dem im quasistationären Betrieb nur kleine Rohrschwingungsamplituden auftreten. Die Nichtberücksichtigung dieses Falles würde im Hochlaufen einen Programmabsturz verursachen, obwohl der eigentlich zu untersuchende Betriebsfall durchaus sinnvoll gewählt gewesen wäre. Die Berücksichtigung des Anschlagens der Rohre untereinander spart daher insbesondere Rechenzeit.

\subsubsection{Iterative Lösung des Gleichungssystems}

Da es sich bei der Lösungsmatrix für die Rohrbewegung um eine Diagonalmatrix mit Hauptachsendominanz handelt, kann für die Lösung der Gleichung (2-72) ein iteratives Verfahren angewandt werden. Für große Gleichungssysteme ist eine iterative Lösung effizienter, da weniger Rechenoperationen als bei der direkten Lösung durchzuführen sind und zudem die Matrizen nicht gespeichert werden müssen. Für einen regulären Knoten erhält man die iterative Lösungsformel

$$
w_{I}^{n+1}=\frac{b_{I}^{n+1}+\Delta t^{2} K_{0}\left(-w_{I-2}^{n+1}+4 w_{I-1}^{n+1}+4 w_{I+1}^{n+1}-w_{I+2}^{n+1}\right)+\Delta t^{2} K_{1}\left(w_{I-1}^{n+1}+w_{I+1}^{n+1}\right)}{4 m+2 \Delta t \zeta+6 \Delta t^{2} K_{0}+2 \Delta t^{2} K_{1}}
$$

für die beiden Knoten rechts vom linken Lager gemäß Abbildung 2-8

$$
\begin{aligned}
& w_{1}^{n+1}=\frac{b_{1}^{n+1}+\Delta t^{2} K_{0}\left(4 w_{2}^{n+1}-w_{3}^{n+1}\right)+\Delta t^{2} K_{1} w_{2}^{n+1}}{4 m+2 \Delta t \zeta+\lambda_{L} \Delta t^{2} K_{0}+2 \Delta t^{2} K_{1}} \\
& w_{2}^{n+1}=\frac{b_{2}^{n+1}+\Delta t^{2} K_{0}\left(4 w_{1}^{n+1}+4 w_{3}^{n+1}-w_{4}^{n+1}\right)+\Delta t^{2} K_{1}\left(w_{1}^{n+1}+w_{3}^{n+1}\right)}{4 m+2 \Delta t \zeta+6 \Delta t^{2} K_{0}+2 \Delta t^{2} K_{1}}
\end{aligned}
$$

analog dazu für die beiden Knoten links vom rechten Lager gemäß Abbildung $2-8$ 


$$
\begin{aligned}
& w_{n_{x}}^{n+1}=\frac{b_{n_{x}}^{n+1}+\Delta t^{2} K_{0}\left(4 w_{n_{x}-1}^{n+1}-w_{n_{x}-2}^{n+1}\right)+\Delta t^{2} K_{1} w_{n_{x}-1}^{n+1}}{4 m+2 \Delta t \zeta+\lambda_{R} \Delta t^{2} K_{0}+2 \Delta t^{2} K_{1}} \\
& w_{n_{x}-1}^{n+1}=\frac{b_{n_{x}-1}^{n+1}+\Delta t^{2} K_{0}\left(4 w_{n_{x}}^{n+1}+4 w_{n_{x}-2}^{n+1}-w_{n_{x}-3}^{n+1}\right)+\Delta t^{2} K_{1}\left(w_{n_{x}-2}^{n+1}+w_{n_{x}}^{n+1}\right)}{4 m+2 \Delta t \zeta+6 \Delta t^{2} K_{0}+2 \Delta t^{2} K_{1}}
\end{aligned}
$$

sowie für die beiden Knoten rechts und links von einem Spitzenlager

$$
\begin{aligned}
& w_{s-1}^{n+1}=\frac{b_{s-1}^{n+1}+\Delta t^{2} K_{0}\left(-w_{s-3}^{n+1}+5 w_{s-2}^{n+1}+6 w_{s}^{n+1}-w_{s+2}^{n+1}\right)+\Delta t^{2} K_{1}\left(w_{s-2}^{n+1}+w_{s}^{n+1}\right)}{4 m+2 \Delta t \zeta+9 \Delta t^{2} K_{0}+2 \Delta t^{2} K_{1}} \\
& w_{s+1}^{n+1}=\frac{b_{s+1}^{n+1}+\Delta t^{2} K_{0}\left(-w_{s-2}^{n+1}+6 w_{s}^{n+1}+5 w_{s+2}^{n+1}-w_{s+3}^{n+1}\right)+\Delta t^{2} K_{1}\left(w_{s}^{n+1}+w_{s+2}^{n+1}\right)}{4 m+2 \Delta t \zeta+9 \Delta t^{2} K_{0}+2 \Delta t^{2} K_{1}}
\end{aligned}
$$

wobei

$$
b_{I}^{n+1}=\Delta t^{2} q_{I}^{n+1}+(4 m+2 \zeta \Delta t) w_{I}^{n}+\left(4 m \Delta t+\zeta \Delta t^{2}\right) \dot{w}_{I}^{n}+\Delta t^{2} m \ddot{w}_{I}^{n}
$$

und

$$
K_{0}=\frac{E I}{\Delta x^{4}} \quad K_{1}=\frac{F}{\Delta x^{2}}
$$

gilt. Die Berechnungsmethode ist implizit, da die neuen Größen nicht nur von den bekannten Größen des vorangegangenen Zeitschritts, sondern auch von den noch unbekannten Größen des neuen Zeitschritts abhängen. Zur Lösung wird das Gauss-Seidel-Point-By-Point-Verfahren verwendet.

Wegen der Verwendung eines iterativen Verfahrens ist es möglich, die Nichtlinearitäten der Kontaktprobleme, die sich aufgrund des Anschlagens der Rohre an die Innenseiten der Bohrungen in den Leitblechen ergeben, durch Kontaktabfrage nach jedem Iterationsschritt mit in die Rechnung einzubringen. Wird aufgrund der Ergebnisse des vorangegangenen Iterationsschritts das Rohr über eine mögliche Lagerstelle hinausbewegt, so wird für diese Lagerstelle im Folgenden die zusätzliche Lagerkraft mit eingerechnet. Stehen Rohr und Leitblech nach einem Iterationsschritt nicht mehr in Kontakt, so wird wieder regulär gerechnet. Allerdings sind für die nichtlinearen Rechnungen kleinere Zeitschrittweiten notwendig als im linearen Fall. Je härter ein Lager modelliert wird, um so kleiner muß der Zeitschritt sein, damit der Auftreffvorgang numerisch aufgelöst wird und Stabilität erreicht werden kann. 
2.2.6 Überprüfung der Modellantwort mit analytischen Formeln

Für die Berechnung von Eigenfrequenzen von Rohren wurden für die vorliegende Arbeit in [Fis00b] analytische Formeln entwickelt. Die Herleitung wird detailiert in Kapitel 3 vorgestellt. Dabei ist die Berücksichtigung von axialen Rohrlasten möglich.

\begin{tabular}{|c|c|c|c|}
\hline$f_{i}[\mathrm{~Hz}]$ & $f_{1}$ & $f_{2}$ & $f_{3}$ \\
\hline Numerisch & 9,5 & 14,0 & 32,0 \\
\hline Analytisch & 9,8 & 14,3 & 31,8 \\
\hline
\end{tabular}

Tabelle 2-3: Vergleich der analytisch und numerisch bestimmten Eigenfrequenzen für einen Beispielfall.

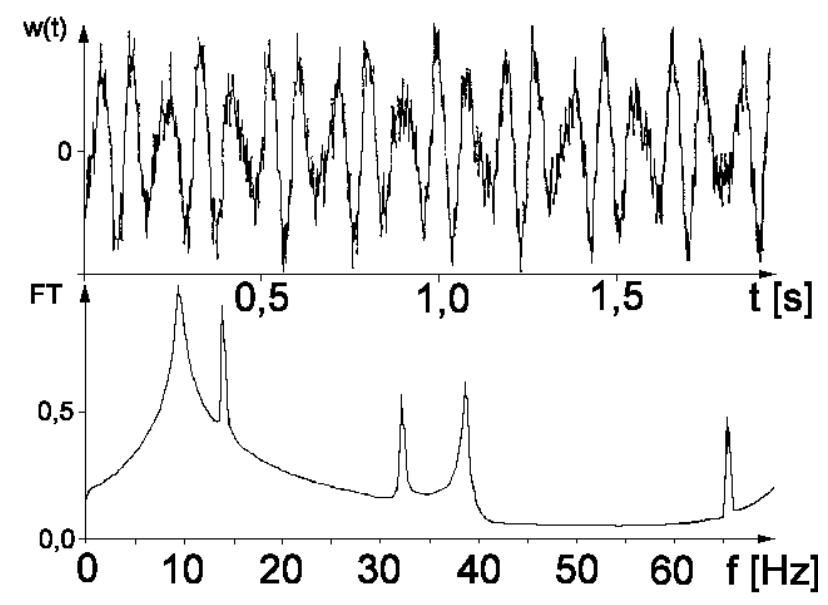

Abbildung 2-11: Simulierte Zeitreihe der Verschiebung mit zugehöriger Fourier-Transformation.

Zur Kontrolle des numerischen Strukturmodells wurde das numerische Modell mit $\zeta=0$ und $q=0$ unter Verwendung einer zufälligen Startbelegung der einzelnen Knoten sich selber überlassen. Die Zeitreihen der sich aus der Simulation ergebenden Verschiebungen wurden dann mit einer Fourier-Transformation analysiert. Die sich ergebenden Eigenfrequenzen können als Peaks der FFTAnalyse entnommen werden. Der Vergleich mit analytisch bestimmten Eigenfrequenzen ergab beste Übereinstimmung im Rahmen der Frequenzauflösung der FFT. Tabelle 2-3 zeigt ein Beispiel. Dasselbe Vorgehen wurde bei verschiedenen Axiallasten $F$ und verschiedenen Stützanordnungen getestet und ergab jedesmal gute Übereinstimmung im Frequenzverhalten. Abbildung 2-11 zeigt 
beispielhaft einen der getesten Fälle für steife Einspannung. Dieser Fall war auch in Abbildung 2-9 benutzt worden, um die Abhängigkeit der Eigenfrequenzen vom Lagerungsfaktor zu zeigen. Die amplitudenbestimmende Steifigkeit des Systems wurde in statischer Rechnung überprüft. Hierzu wurden verschiedene Querlasten auf das Modell aufgegeben und die statischen Verschiebungen mit analytischen Lösungen für Biegebalken verglichen. Auch hier ergab sich sehr gute Übereinstimmung.

Mit Hilfe der Verwendung analytischer Gleichungen für die Eigenfrequenzen der Rohre sowie für die Verschiebungen eines Balkens unter verschiedenen Lagerungsbedingungen war es damit möglich, das für die vorliegende Arbeit entwickelte Strukturmodul abschließend zu validieren. Damit kann zur Kopplung von Strömungs- und Strukturmodell übergegangen werden.

\subsection{Kopplung von Strömung und Struktur}

\subsubsection{Zellüberdeckungsgrad}

Zur Kopplung des Strömungsfeldes mit Antwort der Rohrstruktur wurde für die vorliegende Arbeit der sogenannte Zellüberdeckungsgrad eingeführt. Er berechnet sich aus dem Anteil einer Strömungszelle, die von einem Teil der Rohrstruktur überdeckt wird. Für die betrachtete Zelle werden die strömungsmechanische und die strukturmechanische Lösung für die Geschwindigkeit berechnet und mit Hilfe des Zellüberdeckungsgrades gewichtet. Das Prinzip ist in Abbildung 2-12 verdeutlicht.
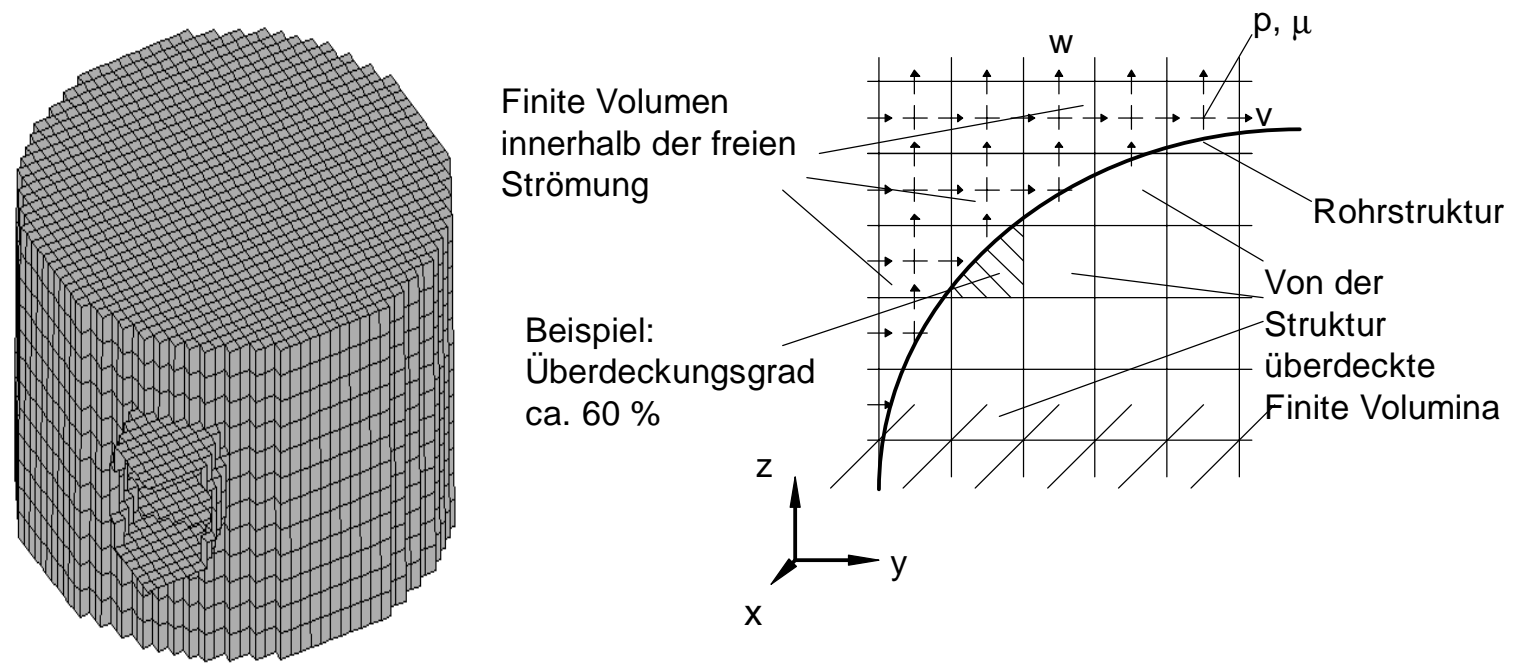

Abbildung 2-12: Kartesisches Berechnungsgitter für Wärmetauschermodell und Definition des Zellüberdeckungsgrads. 
Durch Verwendung dieser besonderen für dieses Verfahren entwickelten Diskretisierungstechnik müssen die Grundgleichungen für die Beschreibung der Strömungs- und Strukturmechanik nicht aus dem kartesischen Koordinatensystem heraustransformiert werden. Dies führte zu einer deutlichen Speicherplatzund Rechenzeitersparnis sowie zu verkürztem Programmcode. Vorteilhaft ist ebenfalls, daß die Rohre sich beliebig nahe kommen können, ohne daß ein Gitter soweit verzerrt würde, daß es zu einem Programmabsturz kommen könnte. Die Routinen zur Berechnung des Überdeckungsgrades wurden von Birkner [Bir98] optimiert.

Physikalisch gesehen kommt der Gittermethode entgegen, daß der Ort der Wirbelablösung nur noch an einer der Kanten des treppenförmig dargestellten Rohrs liegen kann. Damit liegt der maximale Fehler bei der Wirbelablösung bereits fest, er beträgt die Hälfte der gewählten Zellkantenlänge [Kas98]. In Rohrachsrichtung wird das Koordinatensystem um einen Faktor $1 / \xi$ gestreckt. Dadurch wird eine große Anzahl von Strömungsebenen eingespart, wodurch der Rechenaufwand sinkt. Für die strukturdynamischen Berechnungen sind ohnehin nur relativ wenige Knoten notwendig.

Die Grundidee von nur teilblockierten Zellen wurde bereits z.B. in [Has90] zur Berechnung der Durchströmung starrer Rohrbündel und in [Miy92] zur Berechnung von Kugelumströmungen eingesetzt. Im Detail werden jedoch von der vorliegenden Diskretisierung verschiedene Techniken verwendet. Kommerzielle Codes, die teilblockierte Zellen verwenden, sind FLOW-3D oder AeroShape. Besonders für fluid-struktur-gekoppelte Probleme mit variabler Geometrie ist der Ansatz von großem Interesse.

\subsubsection{Koppelkräfte}

Als wichtigste Kräfte neben den bisher betrachteten Lasten auf die Knoten der Struktur, die sich aus den Kontaktkräften beim Anschlagen der Rohre in den Leitblechen bzw. als Erweiterung aus den Kontaktkräften beim Anschlagen der Rohre untereinander sowie den hydraulischen Zusatzkräften im Spalt infolge der Verdrängung von Fluidmasse durch die bewegten Rohre ergeben, treten strömungsmechanische Erregerkräfte auf, die sich aus der Aufsummierung aller Druck- und Schubkräfte an der Rohroberfläche 


$$
\begin{array}{ll}
q_{y}^{D}=-\int_{0}^{2 \pi} p\left(r_{I}, \varphi\right) r_{I} \cos \varphi d \varphi & q_{y}^{R}=\left.\mu r_{I} \int_{0}^{2 \pi} \frac{\partial w_{\varphi}}{\partial r}\right|_{r=r_{I}} \sin \varphi d \varphi \\
q_{z}^{D}=-\int_{0}^{2 \pi} p\left(r_{I}, \varphi\right) r_{I} \sin \varphi d \varphi & q_{z}^{R}=-\left.\mu r_{I} \int_{0}^{2 \pi} \frac{\partial w_{\varphi}}{\partial r}\right|_{r=r_{I}} \cos \varphi d \varphi
\end{array}
$$

je Strömungsebene errechnen. Der wesentlich größere Einfluß geht dabei von den Druckkräften aus, die Reibungskräfte haben nur einen kleinen Einfluß auf die Gesamtkraft.

Die Strömungskräfte sind für eine Reihe von Erregermechanismen verantwortlich, so z.B. für Erregung durch Einzelwirbelablösung, fluidelastische Instabilität oder Turbulenzerregung. Im Gegensatz dazu ergeben sich mechanische Anregungen erst durch das Anschlagen der Rohre an die Leitbleche oder untereinander, nachdem bereits ausreichend hohe Strömungskräfte wirksam waren. Die mechanischen Anregungen sind dann im wesentlichen für die Anregung höherer als erster Eigenfrequenzen verantwortlich. Zur Kopplung von Strömung und Struktur wurde für das Programm eine Routine entwickelt, die die auftretenden Strömungskräfte berechnet [Bir98] und zusammen mit den mechanischen Kräften über dieselben Rampenfunktionen auf das Strukturmodell überträgt.

Im Lagerkontakt können die Bewegungsgleichungen in $y$-Richtung und zRichtung aufgrund der Kräfte infolge des Anschlagens der Rohre in den Leitblechen nicht mehr unabhängig voneinander gelöst werden. Vielmehr müssen diese Punkte simultan gelöst werden. Es ergeben sich lokale Systeme mit zwei Gleichungen und den zwei unbekannten Verschiebungen als gesuchten Größen. Dadurch werden die Bewegungen in $y$-Richtung und $z$-Richtung numerisch miteinander gekoppelt, so, wie es physikalisch auch einleuchtend ist. Andere Vorgehensweisen führten für ökonomisch zu wählende Zeitschrittweiten nicht zu einem stabilen Lösungsverhalten.

Für die je Rohrknoten auftretenden Kräfte werden Speicherfelder vorgehalten, so daß die Strukturknotenlasten bis auf den je Zeitschritt über eine Rampenfunktion neu hinzukommenden Anteil konstant bleiben. Dies führt zu einem so stabilen Verhalten des gekoppelten Systems, daß die Schläge von Rohren untereinander Berücksichtigung finden können. 


\subsubsection{Kinematische Größen}

Das Strukturmodell reagiert auf die äußeren mechanischen und strömungsmechanischen Kräfte mit Verschiebungen, Geschwindigkeiten und Beschleunigungen. Die resultierenden Verschiebungen und Geschwindigkeiten müssen auf das Strömungsmodul zurückübertragen werden. Dabei werden die Geschwindigkeiten über den Zellüberdeckungsgrad mit den Strömungsgeschwindigkeiten gewichtet, die Verschiebungen geben dagegen die neue Position des Rohres nach dem betrachteten Iterationsschritt an. Sie gehen daher in die Berechnung des Überdeckungsgrades des nächsten Iterationsschrittes ein. Die Beschleunigungen werden zur Kopplung zwischen Strömung und Struktur nicht direkt benötigt, sind aber physikalisch über das dritte Axiom von Newton implizit in den bereits behandelten Querkräften enthalten.

\subsubsection{Verbesserung des Druckkorrekturverfahrens}

Für die Druckkorrekturgleichung wird in der Literatur [z.B. No193, Schö90] als Randbedingung in der Nähe von Strukturzellen das Fortlassen des zugehörigen Koeffizienten in der Druckkorrekturgleichung (2-46) verlangt (vgl. Abschnitt 2.1.4.2). Im vorliegenden Fall führt dies jedoch bei sich bewegender Rohrstruktur zu Instabilitäten.

Eine Zelle, die eine zeitlang von der Struktur überdeckt war, erfährt während dieser Zeit nach dem in der Literatur beschriebenen Verfahren keinerlei Änderung ihres Druckwertes mehr. Wird diese Zelle aufgrund der Rohrbewegung dann wieder zu einer Strömungszelle, so ist die Startbelegung ihres Druckwertes inzwischen zu schlecht geworden, um durch eine ökonomisch zu wählende Anzahl von Druckiterationen noch zu einer ausreichend genauen neuen Nährung zu kommen. Die Folge ist, daß die aus der Integration des Druckes an der Rohroberfläche ermittelten Strömungskräfte unphysikalisch groß werden, die Rohrstruktur plötzlich sehr stark ausgelenkt wird und das Programm instabil abbricht.

Abhilfe wird dadurch geschaffen, daß den Strukturzellen für die Druckkorrekturgleichung nicht der Koeffizient Null, sondern ein kleiner, von Null verschiedener Koeffizient zugewiesen wird, z.B. 0,1. In der Struktur ist der Massenfehler aus Gleichung (2-46) genau Null, da sich in der Struktur keine Geschwindigkeitsgradienten ergeben. Es ergibt sich damit in der Rohrstruktur eine glatte Verteilung des Druckfeldes entsprechend einer elliptischen Differentialgleichung mit den Druckwerten auf dem Rand der Struktur als Randbedingungen. 
Diese glatte Verteilung stellt eine sehr gute Startbedingung für die Druckkorrektur dar, sobald die Zelle wieder zu einer Strömungszelle wird. Der Fehler für die Druckberechnung an der Rohroberfläche ist klein, da ja der nun Berücksichtigung findende Druckwert im Innern der Rohrstruktur gut mit dem am Rand gesuchten Druckwert übereinstimmt, und da er nur mit einem kleinen Wichtungsfaktor berücksichtigt wird. Zudem ergibt sich das richtige Strömungsfeld im Außenbereich deshalb, weil die Struktur nach jedem Schritt mit der richtigen Geschwindigkeit belegt wird und sich damit automatisch auch am Strukturrand der richtige Massenfehler $b$ für die Anwendung der Druckkorrekturgleichung (2-46) ergibt.

Vorteilhaft bei der beschriebenen Methode ist, daß das Berechnungverfahren bis auf die modifizierte Bestimmung der Koeffizienten der Druckkorrekturgleichung unverändert beibehalten werden kann. Außerdem verbessern sich Konvergenzrate und Stabilität der Druckkorrekturgleichung drastisch.

\subsubsection{Ablauf eines fluid-struktur-gekoppelten Zeitschritts}

Das Iterationsschema für die strömungsmechanische Berechnung wird nun zum Berechnungsschema für einen vollständig gekoppelten Zeitschritt e rweitert:

1. Es liegt ein geschätztes Druck- und Geschwindigkeitsfeld sowie ein geschätzter Zustand der Struktur vor. Bei Rechnungsbeginn sind alle Größen Null, im weiteren Verlauf wird das Strömungsfeld vom letzten Iterationsschritt als Startlösung verwendet.

2. Berechnung des Zellüberdeckungsgrades für alle Strömungszellen.

3. Berechnung der lokal optimierten Smagorinsky-Konstante und daraus Berechnung der turbulenten Viskosität.

4. Berechnung der Geschwindigkeiten aus den Impulsgleichungen.

5. Wichtung zwischen Strömungs- und Strukturgeschwindigkeit.

6. Berechung der Druckkorrekturen und Aktualisierung des Druckfeldes.

7. Verbesserung des Geschwindigkeitsfeldes.

8. Berechnung der Spaltgeschwindigkeiten aus dem Druckfeld und Übertragung in das kartesische Koordinatensystem.

9. Berechnung der strömungsmechanischen Kräfte.

10. Iteration der Strukturverschiebungen unter ständiger Aktualisierung der mechanischen Kräfte und der Spaltkräfte.

11. Berechnung der neuen Strukturgeschwindigkeiten und Beschleun igungen. 
12. Weiter mit 2., bis die vorgegebene Anzahl von Iterationen bzw. das vorgegebene Residuum der Kontinuitätsgleichung innerhalb des Zeitschritts erreicht ist.

13. Aktualisierung der alten Strömungs- und Strukturfelder durch „Weiterschieben“ der Datenfelder um eine Zeitebene. Beginn des neuen Zeitschritts mit 2.

\subsubsection{Berechnungsbeispiele: Schwingender Einzelzylinder in einem Kanal}

Durch die Einführung des Zellüberdeckungsgrades wurden dreidimensional gekoppelte Berechnungen der strömungsinduzierten Schwingungen von querangeströmten Zylindern ermöglicht. Abbildung 7-1 zeigt bei sehr feiner Auflösung die Geschwindigkeitsverteilung im Strömungsfeld hinter einem schwingenden Zylinder, Abbildung 7-2 stellt die Verteilung der Zähigkeit dar. Wegen der „,backscatter“-Fähigkeit des LES-Lilly-Germano-Turbulenzmodells kommen auch niedrigere Zähigkeitswerte als die Stoffviskosität von 0,001 Pas für Wasser vor.

Abbildung 7-3 bis Abbildung 7-6 zeigen jeweils die Spektralverteilung der Strömungskräfte und der Auslenkungen entsprechend der Gleichung (3-6) für die Fourier-Koeffizienten in und quer zur Anströmrichtung bei einem weiteren homogen mit unterschiedlichen Geschwindigkeiten angeströmten Zylinder. Die erste Eigenfrequenz des Modellzylinders beträgt hier 19,5 Hz. Die Anströmgeschwindigkeiten wurden so gewählt, daß bei etwa 1,4 m/s der Lock-In-Effekt auftritt. Aus den Abbildungen erkennt man, daß sich die Frequenzen der Zylinderkräfte in und quer zur Anströmung wie 2:1 verhalten. Dies muß so sein, da mit jeder Einzelwirbelablösung der Zylinder einen Stoß in Strömungsrichtung erfährt, quer dazu sich die Kraftrichtung mit jedem Druckstoß aber umkehrt. Außerdem erkennt man, daß die Struktur hinsichtlich der Bewegung den zur ersten Eigenfrequenz passenden Anteil des Erregerspektrums quasi ausfiltert. Höherfrequente Anteile werden unterdrückt.

\subsection{Stabile numerische Parameter}

Für die sinnvolle Wahl der Diskretisierungsgrößen Zeitschritt und Zellkantenlänge bei der Berechnung von Wärmetauscherdurchströmungen und fluidstruktur-gekoppelten Analysen konnten für die vorliegende Arbeit aufgrund der Erfahrungen aus [Fis00a] erste Empfehlungen gegeben werden, die sich bei der weiteren Arbeit bewährt haben. 


\subsubsection{Das CFL-Kriterium}

Entscheidend für die Stabilität einer numerischen Strömungssimulation ist die Einhaltung des CFL-Kriteriums (Courant-Friedrich-Levy) [Gri95], wonach eine Störung in einer Strömungszelle innerhalb des Zeitschritts nicht den Zellrand erreichen darf. Die Störung pflanzt sich im inkompressiblen Fall mit der Strömungsgeschwindigkeit fort. Für den vorliegenden Fall gilt daher

$$
C F L=\frac{2 u \Delta t}{\Delta}<1
$$

mit $u$ als Strömungsgeschwindigkeit in Zellmitte, Zeitschritt $\Delta t$ und Zellkantenlänge $\Delta$. Die Bedingung gilt entsprechend für die beiden anderen Koordinatenrichtungen. Um die Stabilität der Gesamtrechnung beurteilen zu können, muß die maximale im Strömungsfeld auftretende CFL-Zahl betrachtet werden. Aus dem Kriterium ergeben sich weitreichende Konsequenzen für das Stabilitätsverhalten des Programms.

\subsubsection{Anwendung des CFL-Kriteriums}

Das Programm wird um so weniger instabilitätsgefährdet sein, je kleiner der Zeitschritt ist. Gleichzeitig erhöht sich damit der Rechenaufwand, bis eine vorgegebene Zeit simuliert ist. Der Zeitschritt darf außerdem nicht zu groß werden, damit schwingungserregende Wirbelskalen zeitlich noch aufgelöst werden können. Für Einzelwirbelablösungen kann als Faustformel der wenigstens zur zeitlichen Auflösung notwendige Zeitschritt zu

$$
\Delta t=\frac{D}{4 u_{0}}
$$

angegeben werden, mit $D$ als Rohrdurchmesser und $u_{0}$ als Zuströmgeschwindigkeit vor dem Rohr. Dabei wird von einer Strouhalzahl von 0,2 und einer Auflösung von wenigstens 20 Zeitschritten je Periode ausgegangen. Hier ist also ökonomisch abzuwägen zwischen Stabilität, Güte des Ergebnisses und Rechenzeit.

Die Rechnung wird außerdem um so stabiler, je größer die Zellkantenlänge ist. Gleichzeitig wird aber eine gewisse räumliche Mindestauflösung notwendig sein, um die schwingungserregenden Wirbelskalen auflösen zu können. Auch hier muß dann ökonomisch abgewogen werden zwischen Speicherplatz- und Rechenzeitbedarf sowie Güte des Ergebnisses und Stabilität. Als Faustformel 
kann angegeben werden, daß die Zellkantenlänge aus physikalischen Gründen höchstens 1/6 des Rohrdurchmessers betragen sollte. Dies war Ergebnis der numerischen Kräftesimulationen am starren Einzelzylinder gewesen.

Je größer die lokale Geschwindigkeit ist, um so instabilitätsgefährdeter wird die numerische Rechnung sein. Die größten lokalen Geschwindigkeiten werden in den Gassen der ersten Rohrreihe nach dem Zustromstutzen bzw. in den Gassen der letzten Rohrreihe vor dem Abströmstutzen auftreten. Die Spaltgeschwindigkeiten zwischen Leitblechen und Rohren sowie die lokalen Geschwindigkeiten bei der Umlenkung der Strömung durch die Leitbleche fallen geringer aus. Die damit für eine Vorabschätzung eines sinnvollen Zeitschritts zugrunde zu legende Spaltgeschwindigkeit berechnet sich aus der Kontinuität sgleichung zu

$$
u_{s p}=u_{0} \frac{T}{T-D}
$$

mit $u_{0}$ als Strömungsgeschwindigkeit im Stutzen, $T$ als Querteilung des Bündels und $D$ als Durchmesser der Rohre.

\section{$\underline{2.4 .3}$ Beispielrechnung}

Als Beispiel für die sinnvolle Vorabfestlegung der Diskretisierungsgrößen soll ein Stutzen mit 100 mm Durchmesser betrachtet werden, der auf ein Rohrbündel mit 20 mm Querteilung aus Rohren mit 12 mm Durchmesser trifft. Der Volumenstrom betrage $40 \mathrm{~m}^{3} / \mathrm{h}$. Die Stutzengeschwindigkeit berechnet sich damit zu $u_{0}=4 \cdot 40 /\left(3600 \cdot \pi \cdot 0,1^{2}\right)=1,42 \mathrm{~m} / \mathrm{s}$. Aus physikalischen Gründen wird damit ein Zeitschritt von $\Delta t<0,012 /(4 \cdot 1,42)=0,0021 \mathrm{~s}$ benötigt. Im Spalt nach dem Stutzen ergibt sich $u_{s p}=1,42 \cdot 20 /(20-12)=3,55 \mathrm{~m} / \mathrm{s}$. Die Zellkantenlänge soll höchstens $\Delta=12 / 6=2 \mathrm{~mm}$ betragen. Das CFL-Kriterium gibt damit einen Zeitschritt von $\Delta t<0,002 /(2 \cdot 3,55)=0,00028$ s vor. Gewählt wird mit einem Sicherheitsfaktor von ca. 0,7 ein Zeitschritt von 0,0002 s.

Für die Berechnungsfälle in der vorliegenden Arbeit war immer das numerisch begründete CFL-Kriterium ausschlaggebend, es sind aber auch Fälle denkbar, bei denen das physikalische Kriterium ausschlaggebend sein könnte. Soweit die im Beispiel dargelegte Vorabschätzung zur Wahl von Zellkantenlänge und Zeitschrittweite eingehalten wurde, wurde kein einziger strömungsmechanisch begründbarer Programmabsturz festgestellt. 


\subsubsection{Stabilität des LES-Modells}

Das verwendete LES-Turbulenzmodell enthält nach Germano und Lilly eine lokal optimierte Smagorinsky-Konstante. Ihre zeitlichen Änderungen erweisen sich nach Literatur als relativ träge, sobald einmal negative Werte erreicht werden. Dies kann nach Literatur zu instabilem Rechnungsverlauf führen [Ber96]. Deshalb wurde im vorliegenden Projekt die Lilly-Germano-Optimierung nur angewendet, wenn das Residuum der Massenerhaltung von einem Iterationsschritt zum nächsten relativ kleiner geworden ist. Sonst wird ein positiver konstanter Wert gesetzt. Positive Werte für die Smagorinsky-Konstante erhöhen die Diffusion und führen zu stabilem Berechnungsverlauf. Nachdem zum Ende der Iteration eines Zeitschritts das Residuum immer sinkt, wird eine korrekt nach Germano und Lilly optimierte turbulente Zähigkeit als Lösung für den betrachteten Zeitschritt erhalten, der Berechnungsverlauf bleibt aber stabil. Alle weiteren in der Literatur genannten Beschränkungen der Konstante zur Stabilisierung (,clipping“) wurden ebenfalls berücksichtigt.

Eine weitere Beschränkung gibt das Turbulenzmodell zur Einhaltung sinnvoller Turbulenzgrößen vor. Demnach soll das größte Seitenverhältnis zwischen zwei Kanten der gleichen Strömungszelle höchstens 1:20 betragen [Fri94]. Daher darf die Streckung der Zellen in Rohrlängsachsenrichtung ebenfalls nur 1:20 betragen, andere Streckungen kommen aufgrund des kartesischen Gitters ja nicht vor. Im obigen Beispiel dürfte also die Zellkantenlänge in Rohrlängsachsenrichtung höchstens $20 \cdot 2=40 \mathrm{~mm}$ betragen. Um ein Bündel mit 1,6 m freier Rohrlänge zu diskretisieren, wären demnach 40 Zellen in Rohrlängsachsenrichtung notwendig. Bei einem Rohrbündeldurchmesser von $350 \mathrm{~mm}$ ergäben sich demnach 40 x 175 x $175=1225000$ Strömungszellen. Dieses Kriterium konnte aufgrund des hohen Rechenaufwandes jedoch nicht eingehalten werden.

\subsubsection{Stabilität des Strukturmodells}

Das einfache Strukturmodell gibt leicht einzuhaltende Bedingungen vor. Die Zeitschrittweite darf bei Verwendung des Newmark-Algorithmus höchstens das 0,318-fache der kürzesten vorkommenden Periodenlänge betragen, um bei Verwendung der Standardwerte unbegrenzte Stabilität zu garantieren. Aufgrund der von CFL schon vorgegebenen Zeitschrittweiten wird diese Beschränkung keine praktischen Auswirkungen haben. 
Die Newmark-Parameter können auch andere Werte als die Standardwerte annehmen. Gleichung (2-72) muß dann entsprechend angepaßt werden. Es muß aber immer $\alpha \geq 1 / 4$ sein. Für diesen Fall wird für optimale Stabilität

$$
\delta=\frac{1}{4}\left(\alpha+\frac{1}{2}\right)^{2}
$$

empfohlen [MAR99]. Hierbei ergibt sich dann ein Dämpfungsfehler und ein Periodenfehler, deren Auswirkungen aber gering sind und in [Bat86] ausführlich diskutiert werden. Die Variation von $\alpha$ und $\delta$ wird zur Verhinderung von unphysikalischen Oszillationen im zeitlichen Beschleunigungsverlauf verwendet [MAR99].

Schwieriger werden die Verhältnisse bei Kontakt zwischen Leitblech und Rohren. Der Kontaktvorgang muß zeitlich aufgelöst werden. Bei weniger elastischen Kontakten wird eine kürzere Kontaktzeit erreicht. Deshalb muß die zeitliche Auflösung auch genauer werden. Bei den verwendeten Materialien konnte mit Zeitschritten, die zu einer CFL-Zahl von 0,2 bis 0,5 führten, auch für das Kontaktmodell stabiles Verhalten erreicht werden. Der Zeitschritt kann erhöht werden, wenn die Lateralsteifigkeit der Rohre herabgesetzt wird.

\subsubsection{Fluid-Struktur-Effekte}

Im Zusammenhang mit den Kontaktkräften zwischen Rohren und Leitblechen wurde ein Effekt festgestellt, der lokal zu CFL-Zahlen größer als Eins führen kann. Findet nämlich ein harter Schlag zwischen Rohr und Leitblech statt, wird das Rohr auch zu hochfrequenten Schwingungen angeregt. Diese Schwingungen führen zwar nur zu kleinen Amplituden, jedoch zu hohen Schwingungsgeschwindigkeiten. Aufgrund der Kopplung zwischen Rohrgeschwindigkeit und Strömungsfeld wird auch die rohrbenachbarte Strömungszelle lokal eine hohe Geschwindigkeit aufweisen und lokal zu einer hohen CFL-Zahl führen.

Abhilfe hat zum einen ein modifizierter Gewichtungsansatz zwischen Strömungs- und Strukturzelle gebracht. Soweit diese Maßnahme nicht ausreichend ist, kann der Zeitschritt weiter verkleinert werden, was allerdings mehrere Versuche und eine längere Rechenzeit erfordert. Außerdem kann auch hier die Lateralsteifigkeit der Rohre herabgesetzt werden. Dadurch werden höhere Rohreigenfrequenzen durch das Anschlagen nicht mehr so stark angeregt. Hier ist zu entscheiden, bis zu welcher Eigenfrequenz die Güte des Ergebnisses hoch sein soll. Auch hier werden evtl. mehrere Versuche notwendig sein, jedoch führt diese Maßnahme zum stabilsten Programmverhalten. In der vorliegenden Arbeit 
haben sich die dritte und sechste Eigenfrequenz in manchen Fällen als dominierend herausgestellt. Entsprechend wurde die Lateralsteifigkeit so herabgesetzt, daß höhere Eigenfrequenzen nicht mehr dominant im Spektrum auftauchten.

Ein weiterer Effekt, der die Strömungsmechanik erst im struktur-gekoppelten Rechenbetrieb negativ beeinflußt, ist der, daß aufgrund der Seitwärtsschwingungen der Rohre lokal die Verengungen zwischen zwei benachbarten Rohren noch kleiner werden können, wodurch die lokale Spaltgeschwindigkeit noch ansteigt. Die CFL-Zahl steigt dadurch ebenfalls an. Findet dieser Vorgang in Stutzennähe statt, kann $C F L$ auch größer als Eins werden und zum Programmabsturz führen. Abhilfe schafft hier ein Zeitschritt, der nicht mit der geometrischen Teilung des Rohrbodens vorab berechnet wird, sondern mit einer kleineren Teilung entsprechend der maximal zu erwartenden Rohrauslenkung. Dadurch wird die maximal zu erwartende lokale Strömungsgeschwindigkeit höher geschätzt, entsprechend ergibt sich ein kleinerer Zeitschritt mit längerer Rechenzeit. Bei sich stark annährenden Rohren sinkt CFL wieder ab, da sich aufgrund von Reibungseffekten nur noch kleine Geschwindigkeiten ergeben.

Bei Berücksichtigung dieser weiteren beiden in der fluid-struktur-gekoppelten Simulation begründeten Effekte konnten die Programmläufe bis hin zu Kontaktvorgängen zwischen schwingenden Rohren stabil gehalten werden.

\subsection{Implementierung}

Das Programm FIVSIC-3D, das innerhalb dieser Arbeit entwickelt wurde, wurde in der Programmiersprache Fortran 90 [RRZ93, Mic95] geschrieben. Der Solver verwendet dabei nur Standard-Fortran 90 [RRZ93] und ist somit auf jeder Plattform compilierbar und lauffähig, insbesondere auch auf Großrechnern und UNIX-Workstations.

Der Post-Processor POST.EXE zur Erzeugung grafischer Darstellungen der Strömungsgrößen wurde in der Variante Fortran PowerStation 4.0 [Mic95] für das Betriebssystem Windows NT 4.0 geschrieben. Die Benutzung grafischer Befehle bedingt es, daß dieser Teil des Programmsystems nicht ohne Änderungen in den grafischen Unterroutinen auf andere Plattformen portierbar ist. Hier muß die grafische Schnittstelle jeweils angepaßt werden.

Dies gilt auch für das Eingabeprogramm. Für die Eingabe wurde eine grafische Benutzeroberfläche entwickelt [Bau01], die es gestattet, unter Anwendung einer Menüstruktur alle notwendigen Dateneingaben zu tätigen. Zur Kontrolle wird die Wirkung einer Eingabe nach Bestätigung in einer stilisierten Grafik ver- 
deutlicht. Ein Bildschirmabzug der grafischen Bedienoberfläche ist in Abbildung 2-13 zu sehen.

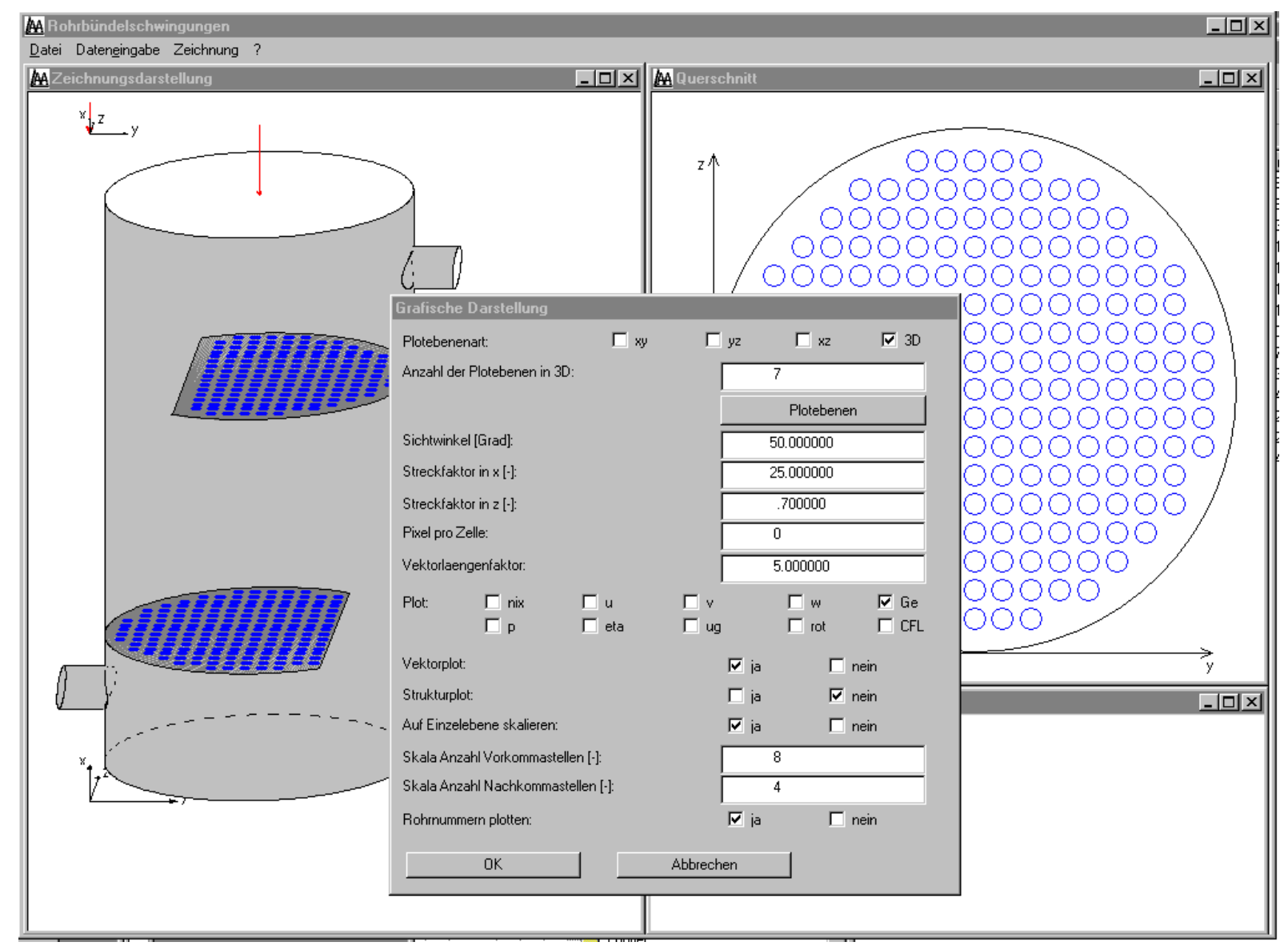

Abbildung 2-13: Beispiel eines Bildschirmabzugs des Eingabeprogramms.

Das Ablaufdiagramm des Gesamtsystems ist in Abbildung 2-14 zu sehen. Die grafische Bedienoberfläche EINGABE.EXE erzeugt die Eingabedatei FIV.ST für den Solver FIVSIC-3D.EXE. Diese problembeschreibende Datei kann im ASCII-Format auf jede NT- oder UNIX-Plattform portiert werden. Der Solver erzeugt die Lösungsdatei RES*.DAT. Dabei steht * für die Nummer der Lösung. Die Lösungsdatei wird vom Postprocessor POST.EXE zur grafischen Darstellung der Ergebnisse und für die Anfertigung von Filmen verwendet.

Der Solver erzeugt eine Reihe weiterer Datenausgaben, so die Dateien KRAEFTE.DAT, die die Gesamtkraefte auf ein ausgewähltes Rohr enthält, ERGEBNIS.DAT, die für einen bestimmten Rohrknoten die kinematischen Größen im Zeitverlauf enthält, die Dateien BESCHL*ASC, die für bis zu 12 Rohre die kinematischen Größen an einer beliebigen Anzahl von Meßtiefen enthält, MINMAX.SYS, die für die Anfertigung von Filmen benötigt wird, 
VERSCHLEISS.SYS, die für die Verschleißauswertung an den Lagerstellen benötigt wird, sowie FIV_PROT.SYS, die ein Berechnungsprotokoll des Simulationslaufs enthält.

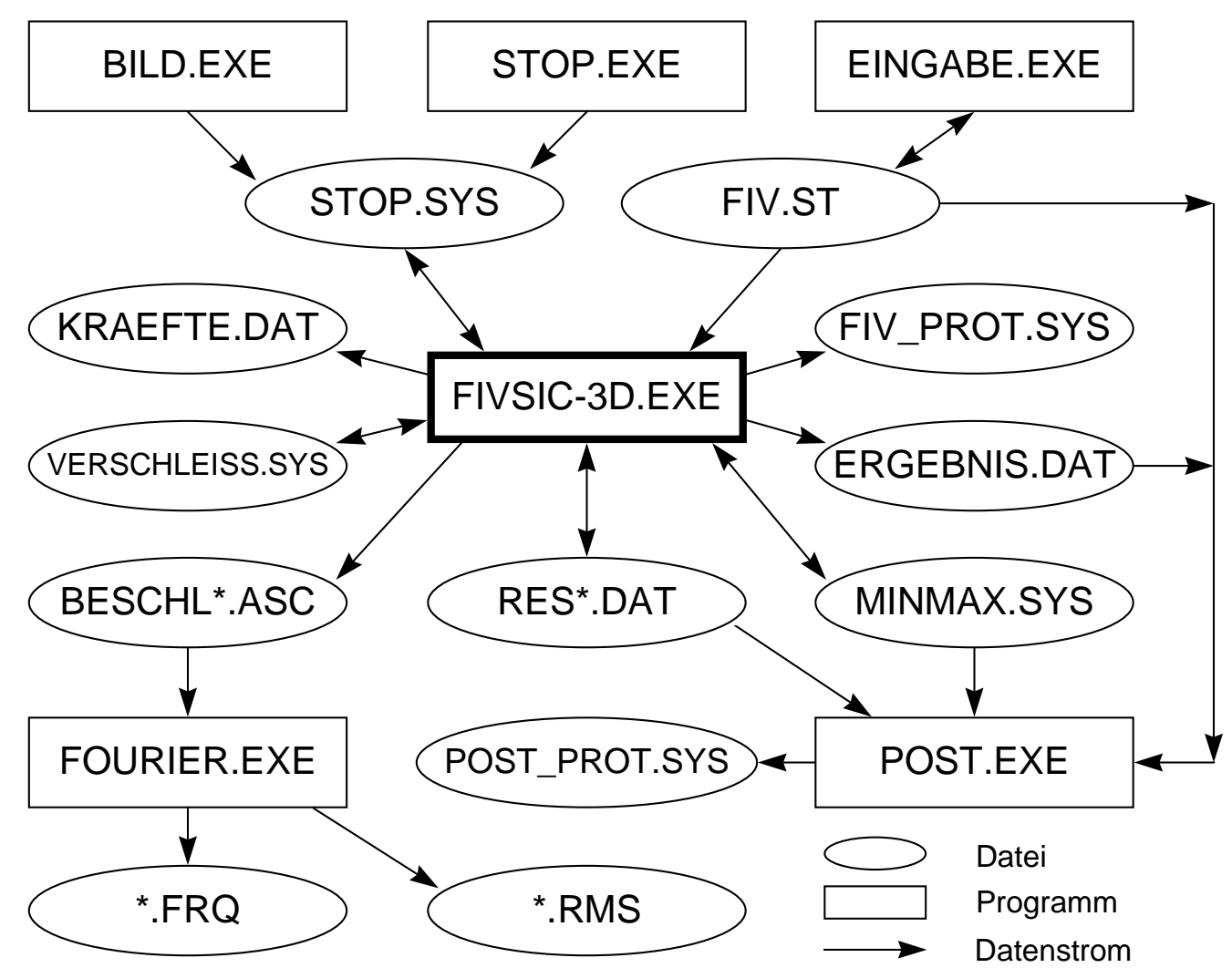

Abbildung 2-14: Ablaufschema des Programmsystems um FIVSIC-3D.

Über die Programme BILD.EXE und STOP.EXE können Befehle an das laufende Programm übermittelt werden. BILD.EXE notiert in der Datei STOP.SYS, daß nach Ende des laufenden Zeitschritts eine Ergebnisdatei RES*.DAT geschrieben werden soll, die dann bei weiterlaufendem Solver von POST.EXE ausgewertet werden kann. POST.EXE verwendet die vom Solver berechneten Ergebnisse und die Eingabedaten. Ein Protokoll der Auswertung wird in POST_PROT.SYS festgehalten. STOP.EXE teilt dem Solver mit, daß nach Abschluß des laufenden Zeitschritts das Programm regulär beendet werden soll.

Die in den Dateien BESCHL*.ASC enthaltenen kinematischen Daten werden vom Programm FOURIER.EXE verwendet, um Fourier-Transformationen und RMS-Berechnungen von Zeitreihen vorzunehmen. Die Daten werden in die Dateien *.FRQ und *.RMS ausgelesen. Diese Dateitypen können von Microsoft 
Excel gelesen werden, um grafische Darstellungen $\mathrm{zu}$ erzeugen. FOURIER.EXE wird von der Datei AUSWERT.ST gesteuert, die in Abbildung 2-14 der Übersichtlichkeit wegen nicht mehr dargestellt ist. FOURIER.EXE wird ebenfalls zur Auswertung der Meßwerte eingesetzt. 


\section{Experimentelle Untersuchungen}

Um das in Kapitel 2 entwickelte numerische Berechnungsverfahren überprüfen zu können, wurde ein Versuchskanal errichtet, in dem konstruktiv realistisch ausgeführte Rohrbündel strömungsinduziert zu Schwingungen angeregt und vermessen werden konnten.

\subsection{Beschreibung der Versuchseinrichtung}

\subsection{1 Übersicht über den Versuchsstand}

Der Versuchsstand besteht aus zwei Ästen eines geschlossenen Kreislaufs mit einem maximal zulässigen Betriebsdruck von 5 bar bei Raumtemperatur. Abbildung 3-1 zeigt eine Übersichtszeichnung.
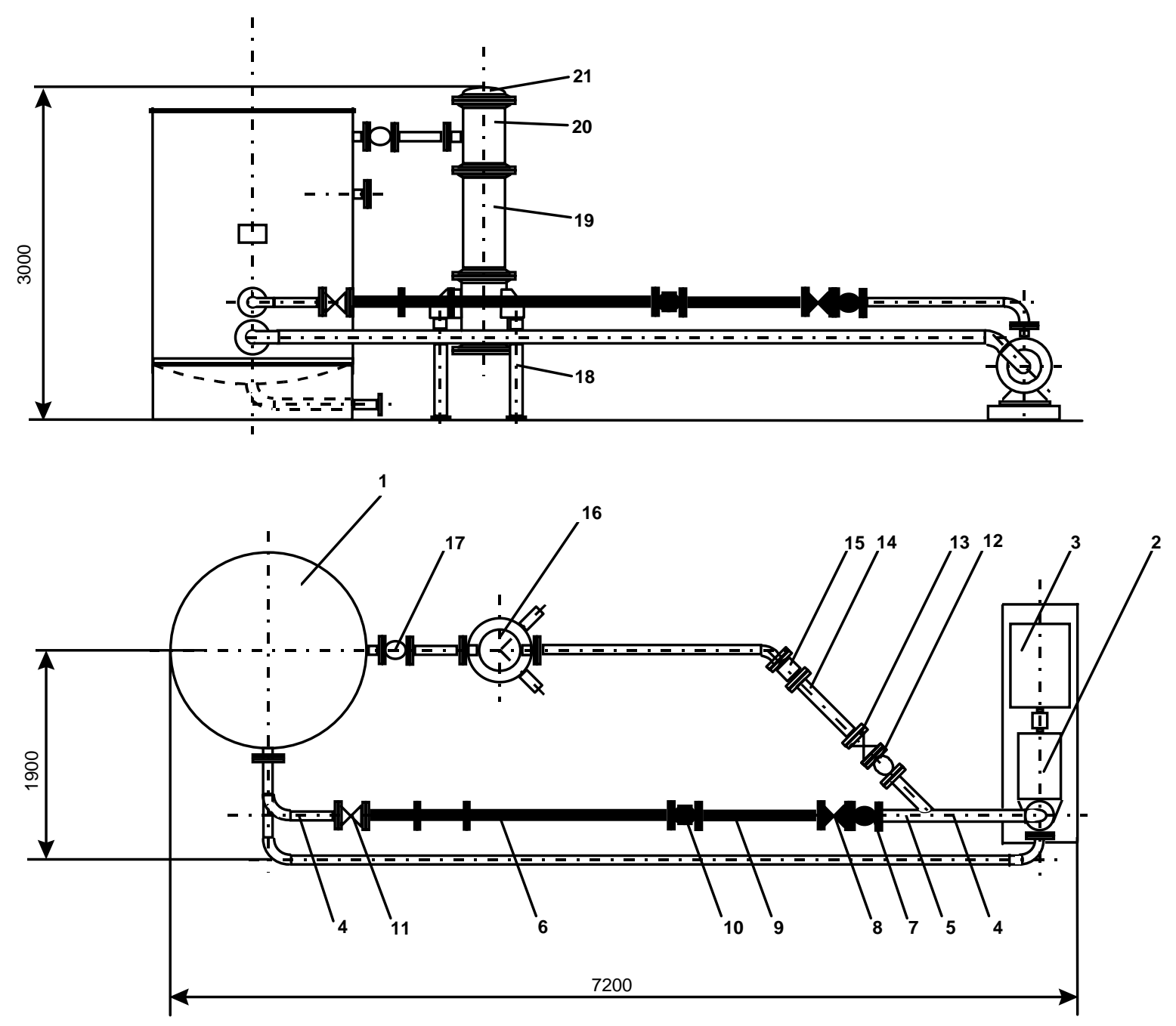

Abbildung 3-1: Übersichtszeichnung zum Versuchsstand. 
Von einem Stutzen DN 125 eines Ausgleichsbehälters (1) mit ca. $4 \mathrm{~m}^{3}$ Inhalt strömt Wasser über eine Zulaufleitung DN 125 zur Radialkreiselpumpe (2) (Typ ETANORM, Firma KSB), die über einen 45-kW-Drehstrommotor (3) angetrieben und deren Drehzahl mittels eines Frequenzumrichters eingestellt wird. Der zu fördernde Volumenstrom der Pumpe kann zwischen 40 und $250 \mathrm{~m}^{3} / \mathrm{h}$ stufenlos verändert werden. Nach der Pumpe reduziert sich der Nenndurchmesser auf DN 100 und die Rohrleitung verzweigt sich (4).

Im durchfließenden Ast wird der Nenndurchmesser zunächst auf DN 80 reduziert (5), damit die darauffolgenden Komponenten der alten Meßstrecke (6) weiterverwendet werden können. Ein Gummibalgkompensator DN 80 (7) reduziert Systemschwingungen und gleicht z.B. thermisch verursachte Längenänderungen des Stranges aus. Daran anschließend befindet sich ein Stellventil DN 80 (8), so daß der Zulauf zur alten Meßstrecke versperrt werden kann. Nach einer Beruhigungsstrecke DN 80 (9) wird der Durchfluß über ein Flow-Meter DN 80 (10) gemessen, bevor das Fluid durch die bereits vorhandene Meßstrecke (6) geführt wird. Die Positionen (6) bis (10) waren bereits für die Arbeiten von Kassera [Kas94, Kas97a] vorhanden. Ein zweites Stellventil DN 100 (11) ermöglicht es, die alte Meßstrecke vom Ausgleichsbehälter (1) abzusperren. Der Rücklaufstutzen DN 100 liegt unterhalb der Wasserlinie des Ausgleichsbehälters. Durch die Versperrung der beiden Ventile (8) und (11) kann die alte Meßstrecke vollständig vom übrigen Strömungskreislauf abgetrennt werden, um dort Arbeiten durchführen zu können, auch wenn der Rest der Anlage in Betrieb ist.

Im abzweigenden Ast DN 100 folgt sofort ein Kompensator DN 100 (12), danach ein weiteres Absperrventil DN 100 (13, wie 11), eine Beruhigungsstrecke (14) und ein digitales magnetisch-induktives Flow-Meter DN 100 (15) der Firma Endress und Hauser. Der Absperrschieber (13) ist besonders druckverlustarm und wenig turbulenzerzeugend ausgeführt. Daran anschließend wird die Strömung in die neue Meßstrecke (16) eingeleitet. Das Fluid steigt im Wärmetauschermodell bis über die Wasserlinie des Ausgleichsbehälters (1) und wird über einen Zulaufstutzen DN 100 und ein um $45^{\circ}$ gekröpftes Rohr in den Ausgleichsbehälter (1) zurückgeführt. Im abzweigenden Strang genügt daher die Absperrung des Ventils (13), um an der neuen Meßstrecke (16) Arbeiten unabhängig vom Betrieb der alten Meßstrecke (6) durchführen zu können. Im Ablaufrohr DN 100 zwischen der neuen Meßstrecke (16) und dem Ausgleichsbehälter (1) befindet sich ein weiterer Kompensator DN 100 (18). 
Sämtliche Rohrleitungsstücke wurden aus Edelstahl X 10 CrNiMoTi 1810 als Schweißkonstruktionen gefertigt. Die einzelnen Rohrleitungsstücke und Komponenten sind über Flansche DIN 2533 verbunden und gedichtet. Der Aufbau auf Rohrleitungsstützen wurde mit Hilfe geschweißter Vierkant-ProfilTragwerke bewerkstelligt, die am Werkhallenboden aufgestützt wurden. Für die Ermittlung der Meßergebnisse in der vorliegenden Arbeit wurde nur die im folgenden Abschnitt beschriebene neue Meßstrecke verwendet. Abbildung 3-2 zeigt den betriebsbereiten Versuchsstand.

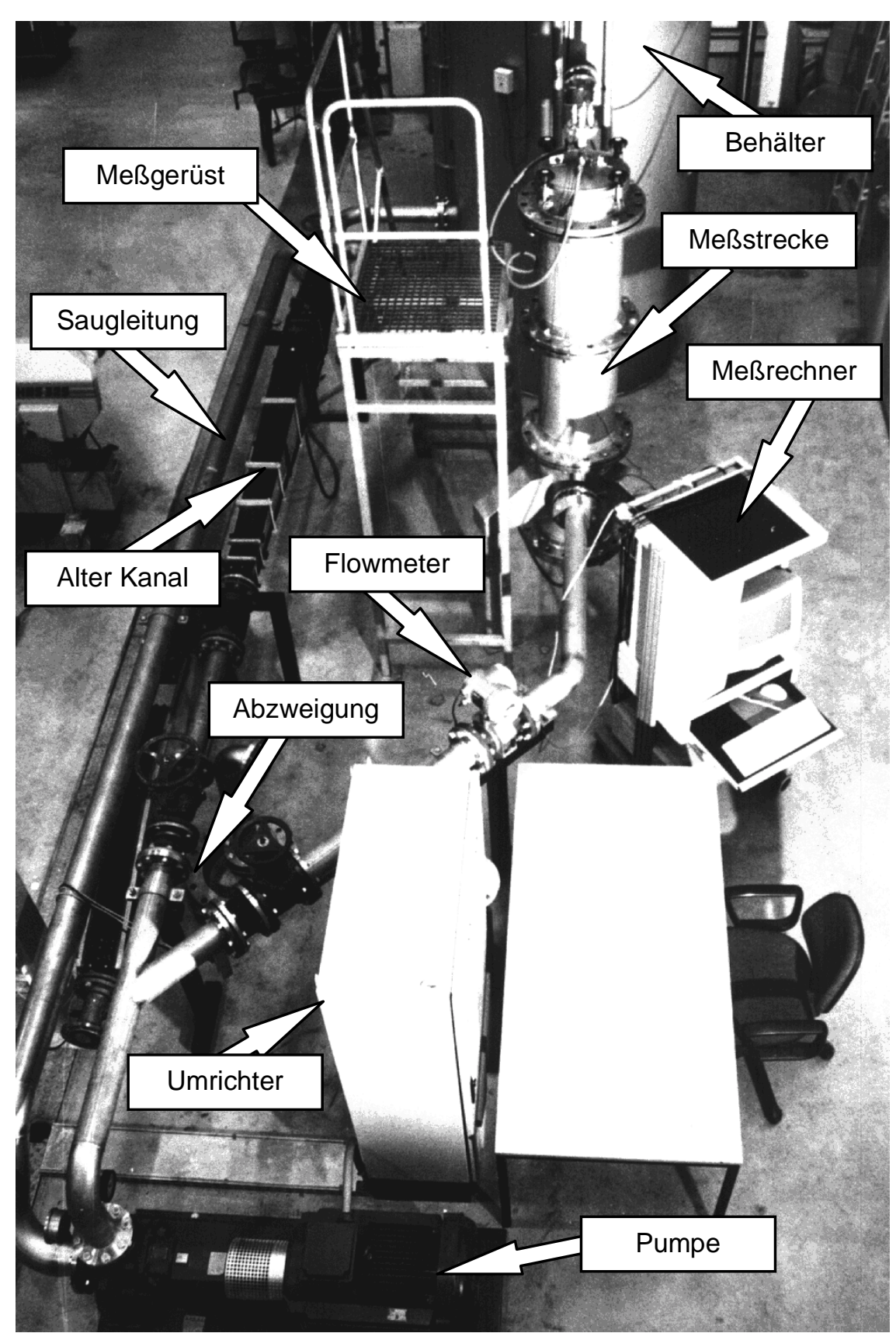

Abbildung 3-2: Betriebsbereiter Versuchsstand. 


\subsubsection{Aufbau der neuen Meßstrecke}

Als neue Meßstrecke fungiert ein realen RohrbündelwärmetauscherKonstruktionen nachempfundener senkrecht stehender Behälter, der modular aufgebaut ist. Das Bodenteil (18) umfaßt einen Dreifuß, an den ein ca. $575 \mathrm{~mm}$ hohes Zylinderteil mit Bodenplatte, Zulaufstutzen und Anschlußflansch geschweißt ist. Das Mittelteil (19) besteht aus einem zylindrischen Körper von 1100 mm Höhe mit zwei Anschlußflanschen, der gegen einen ebensolchen mit nur 600 mm Höhe ausgetauscht werden kann, und das Oberteil (20) aus einem zylindrischen Körper mit Anschlußflansch, Ablaufstutzen und Deckel in Schnellverschlußbauweise (21). Durch den Zulaufstutzen strömt Wasser in den Behälter ein. In der Konfiguration für Schauversuche wird auf das Bodenteil (18) direkt ein Deckel aufgesetzt, der mit Schauglas ausgeführt ist. Das Schwingverhalten der angeströmten Rohre von verkürzten und nur einseitig eingespannten Rohrbündeln kann so direkt beobachtet werden. In der geschlossenen Ausführung werden die Rohrschwingungen mit Piezobeschleunigungssensoren gemessen. Dazu ist der obere Schnellverschluß (21) mit einer Belüftungsöffnung mit Verschlußhahn und mit einer mittels Plastilin und Spannschrauben abdichtbaren Führung versehen, die die Signalkabel der Meßsensorik aufnimmt.

Die modulare Bauweise ermöglicht es, Versuche mit Rohrbündeln der drei verschiedenen freien Rohrlängen $2100 \mathrm{~mm}, 1600 \mathrm{~mm}$ (jeweils doppelt eingespannt) und $500 \mathrm{~mm}$ (nur unten eingespannt) durchzuführen. Alle Komponenten der neuen Meßstrecke sind in Edelstahl X 10 CrNiMoTi 1810 ausgeführt. Zusammen mit der von Kassera [Kas94, Kas97a] verwendeten kleineren Meßstrecke für Prinzipmodelle steht damit ein sehr vielseitig verwendbarer Prüfstand zur Untersuchung von strömungsinduzierten Schwingungen zur Verfügung. Die im Versuch in der neuen Meßstrecke verwendeten Rohrbündel sind im nachfolgenden Abschnitt beschrieben.

\subsubsection{Aufbau der Rohrbündel}

Die verwendeten Rohrbündel sind jeweils 1,7 m und 2,2 m lang, haben eine Querteilung von 19,16 mm und freie Rohrlängen von 1,6 m bzw. 2,1 m. Die Längsteilung der versetzt angeordneten Bündel beträgt 16,59 mm. Die Rohraußendurchmesser betragen $12 \mathrm{~mm}$ und $16 \mathrm{~mm}$. Durch Kombination der drei Parameter ergeben sich insgesamt acht verschiedene Rohrbündel. 
Die Rohrbündel bestehen unten aus einer Bodenplatte aus AlCuMg 1 F39 mit Durchmesser $345 \mathrm{~mm}$ und $50 \mathrm{~mm}$ Stärke, vier Gewindestangen M10 aus Edelstahl X 10 CrNiMoTi 18 10, zwei Leitblechen aus AlMgSi 0,5 F39 mit 345 mm Durchmesser und $20 \mathrm{~mm}$ Stärke, einer oben schwimmend gelagerten Kopfplatte aus AlCuMg 1 F39 mit 345 mm Durchmesser und 50 mm Stärke und ca. 220 bis 250 Rohren in jedem Rohrbündel.

Bei Verwendung eines Edelstahlrings zur Befestigung der Rohrbündel am Bodenflansch des Testbehälters war mit Hilfe einer ICP-Spektroskopie insbesondere das Element Aluminium im Korrosionsprodukt nachgewiesen worden. Mit einem elektrisch leitenden Metallring würden die Rohrbündel gemäß der elektrochemischen Spannungsreihe nach der Gleichung [Kaß80]

$$
2 \mathrm{Al}+6 \mathrm{H}_{2} \mathrm{O} \rightarrow 3 \mathrm{H}_{2}+2 \mathrm{Al}(\mathrm{OH})_{3}
$$

korrodieren. Edelstahlrohrbündel wären hingegen in der Anschaffung etwa dreimal so teuer gewesen. Die Bodenplatte ist daher über acht Schrauben M10 und einen Kunststoffring fest mit dem Bodenflansch des Testbehälters verschraubt. Der Kunststoffring dient als elektrische Trennung zwischen Edelstahlbehälter und legierten Aluminiumrohrbündeln dem Korrosionsschutz der Rohrbündel. Die Gewichtsmenge des entstehenden Korrosionsproduktes konnte so auf einen Bruchteil des Wertes bei Verwendung eines Stahlringes reduziert werden. Die Entwicklung der Gegenmaßnahmen gegen elektrochemische Korrosion ist in [Fis01b] ausführlich beschrieben.

Die Leitbleche wurden mit je einer Mutter M10 oberhalb und unterhalb der Leitbleche in den vorgesehenenen Positionen bei $1 / 3$ und $2 / 3$ der Rohrbündellänge auf den Gewindestangen fixiert. Nachdem die Muttern fest angezogen worden waren, wurden sie mit Sekundenkleber „Loctite“ gesichert. Am oberen Ende der Gewindestangen werden zur radialen Fixierung des Rohrbündels Kunststoffrollen gegen die Behälterwand gepreßt. Die axiale Verschieblichkeit der oberen Rohrplatte ist dadurch nicht behindert. Außerdem kann an den oben herausragenden Enden der Gewindestangen eine Traverse zum Herausheben der bis zu $85 \mathrm{~kg}$ wiegenden Rohrbündel angebracht werden.

Die Leitbleche sind mit Fensterausschnitten von 90 mm Höhe und gemäß DIN 28182 [DIN80] mit Bohrungsübermaßen von 0,7 mm ausgestattet, so daß eine mäanderformige Durchströmung der Rohrbündel nebst einer in der Praxis ebenfalls auftretenden Leckageströmung durch die Spalte zwischen Leitblechen und Rohren gewährleistet ist. Um die Rohre an den Lagerstellen beim häufig wäh- 
rend der Versuchsreihen hervorgerufenen Zustand der instabilen Schwingungsanregung nicht unnötig der Gefahr einer vorschnellen Schädigung auszusetzen, wurden die Leitbleche mit einer Dicke von $20 \mathrm{~mm}$ deutlich stärker ausgeführt, als nach der Praxis des Wärmetauscherbaus eigentlich üblich gewesen wäre. Dadurch wird jedoch die Flächenpressung und damit der dynamische Abrieb der Rohre an den Leitblechen vermindert, weitere Einflüsse auf das Meßvorhaben ergeben sich nicht. Die Gewindestangen wurden so eingesetzt, daß jedes Leitblech durch drei von vier Gewindestangen gelagert ist und somit ein Verkippen infolge der strömungsmechanischen Belastung nicht möglich ist.

Die Rohre sind nahtlos gezogene Präzisionsrohre aus Aluminiumknetlegierung AlMgSi 0,5 F22 mit 1 mm Wandstärke. Sie werden mit Hilfe des Verfahrens des hydraulischen Aufweitens [Kri76, Hö198] in der oberen und unteren Rohrplatte fixiert. Beim hydraulischen Aufweiten werden die Rohrenden in die Bohrung der Rohrplatte gesteckt und mit Hilfe einer Aufweitsonde unter hohem Innendruck radial plastisch verformt. Die Rohrplatte wird teilplastisch verformt und federt nach Wegnahme des Aufweitdrucks elastisch so zurück, daß das Rohr von der Platte fest eingeklemmt wird. Zusätzlich entsteht durch eine leichte Tonnenformbildung am Rohrende ein Formschluß zwischen Rohr und Rohrplatte. Notwendige Voraussetzung für diese Fertigungsart ist, daß der Plattenwerkstoff erst unter höherer Last plastifiziert als der Rohrwerkstoff, die Plastifizierungsgrenze in der Platte nicht die Stege erreicht und der Rohrwerkstoff eine hohe Bruchdehnung besitzt. Für die verwendete Materialkombination sind diese Voraussetzungen gewährleistet. Nachfolgend werden die notwendigen Arbeitsschritte geschildert, um von der laborhaften Verwendung des Verfahrens zu einem fertigungs- und betriebssicheren Vorgehen für die „Serienproduktion“ von hydraulisch gefügten Rohr-Rohrplatten-Verbindungen zu gelangen.

Da für die vorliegende Aufgabe Rohrinnen- und -außenseite im Versuchsstand nicht gegeneinander gedichtet werden müssen, ist die Dichtigkeit der Verbindung ohne Belang. Als Auslegungsgrundlage müssen daher nur die maximal zu erwartenden Rohrausreißkräfte ermittelt werden. Hierzu wurde die Länge der dynamischen Biegelinie zwischen zwei Leitblechen bei einer maximalen Auslenkung der Rohre um die halbe Querteilung in der Mitte errechnet, aus dem Unterschied zur Ausgangslänge die auftretende Dehnung bestimmt, mit dem Elastizitätsmodul auf die Spannung geschlossen und über die Querschnittsfläche eine zu erwartende dynamische Lastspitze der Ausreißkraft von maximal 
775 N (12 mm) bzw. 1050 N (16 mm) pro Rohr berechnet. Unter Berücksichtigung eines im Maschinenbau üblichen Sicherheitsfaktors von 4,0 bei höchsten Anforderungen an die Sicherheit [Nie81] wurden als Auslegungshaltekräfte für die Rohr-Rohrplattenverbindung 3,1 kN bei Verwendung der 12-mm-Rohre bzw. 4,2 kN bei Verwendung der 16-mm-Rohre festgelegt. Die sich dabei rechnerisch ergebende Beanspruchung der Rohre bleibt unterhalb der Streckgrenze des Rohrwerkstoffs.

Im nächsten Schritt mußte der zur Erlangung der erwünschten Rohrausreißkraft notwendige Aufweitdruck rechnerisch bestimmt werden. Die Rohrplatte wird dabei durch eine Ersatzhülse nach dem Konzept von Kohlpaintner [Koh93] repräsentiert. Nach Kohlpaintner kann der Außendurchmesser einer Ersatzhülse nach der Formel

$$
\begin{gathered}
D_{H}=T \cdot\left(12,55009 \kappa^{5}-16,21818 \kappa^{4}+10,88288 \kappa^{3}\right. \\
\left.-2,43388 \kappa^{2}+1,94683 \kappa+0,98034\right)
\end{gathered}
$$

mit $\kappa=(T-D) / T$ so bestimmt werden, daß die Hülse am Bohrungsrand im Mittel die gleiche radiale Verformung aufweist wie der Rand einer realen Rohrplattenbohrung unter Berücksichtigung der Rohrplattenstege. Nach Kohlpaintners Verfahren wurden daraufhin Ersatzhülsenaußendurchmesser von 23,96 mm für das 16-mm-Rohr bzw. 32,16 mm für das 12-mm-Rohr errechnet. Da bei gleicher Teilung bei kleinerem Bohrungsdurchmesser die Stege der Platte steifer werden, muß auch der Ersatzhülsenaußendurchmesser ansteigen.

Eine analytische Abschätzung der Rohrausreißkraft kann aus einer Autofrettagerechnung für Rohr und Ersatzhülse ermittelt werden. In Anlehnung an [Fis99b] ergibt sich die Rohrausreißkraft analytisch zu

$$
F_{A}=\pi D s_{P} \mu_{0} \max \left\{p_{A} \frac{D_{H}^{2}}{D^{2}} \frac{D^{2}-d^{2}}{D_{H}^{2}-d^{2}}-\sigma_{R}^{F} \ln \frac{D}{d}, 0\right\} .
$$

Nimmt man den Haftreibungskoeffizienten mit 0,2 besonders konservativ an [Hö197], ergibt sich ein notwendiger Aufweitdruck von 1124 bar für die 12mm-Rohre bzw. 838 bar für die 16-mm-Rohre. Hierbei wurde der Innendurchmesser mit 10,3 mm (12-mm-Rohr) bzw. 14,28 mm (16-mm-Rohr) bereits so eingesetzt, als ob der Spalt zwischen Rohr und Bohrung durch plastische Verformung unter Erhaltung der Masse des Rohrwerkstoffs bereits geschlossen worden wäre. 
Die Berücksichtigung des Tonneneffektes und der Kontaktspitzen kann mit Hilfe der Methode der Finiten Elemente erfolgen. Hölzl hat im Rahmen seiner Dissertation [Hö198] ein vollautomatisches parametergesteuertes Berechnungswerkzeug für axialsymmetrische Rohr-Rohrhülsen-Verbindungen auf Basis des FE-Paketes MARC/Mentat erstellt. Dabei müssen nur die relevanten Geometrieund Werkstoffparameter einer Steuerdatei an das Problem angepaßt werden. MARC errechnet dann unter Berücksichtigung des Spaltes und der Plastifizierung des Rohres und der Innenzone der Hülse beim Aufweiten die an der Trennfuge herrschende Pressungsverteilung. Multipliziert man die sich ergebende mittlere Pressung mit der Zylinderfläche und dem Haftreibungsbeiwert, so ergibt sich bei einem Haftreibungskoeffizienten von 0,2 ein erforderlicher Aufweitdruck von 1210 bar für die 12-mm-Rohre bzw. 940 bar für die 16-mmRohre.

Für beide Vorberechnungsarten nachteilig ist, daß der genaue Wert des rechnerischen Haftreibungsbeiwertes nicht bekannt ist. Er hängt stark von der Oberflächenbeschaffenheit der Bohrung ab. Daher waren zur genauen Bestimmung des notwendigen Aufweitdrucks Ausreißversuche unabdingbar. Allerdings konnten die berechneten Werte als gute Anhaltspunkte für den zu erwartenden Meßbereich dienen. Als Startwert für den Aufweitdruck wurde das arithmetische Mittel der aus beiden Berechnungsarten ermittelten Aufweitdrücke verwendet. Abhängig von den Meßergebnissen wurde der Aufweitdruck so variiert, daß ein repräsentatives Meßergebnis erreicht werden konnte.

Zur Herstellung der Verbindung mußten zwei Aufweitsonden aus Baustahl St52 hergestellt werden. Diese bestehen aus einem Dorn mit gegenüber den Rohrinnenmaßen von 10 und $14 \mathrm{~mm}$ um ein bis zwei zehntel Millimeter reduzierten Außendurchmessern. In der Achse des Dorns verläuft eine zentrale Sackbohrung $\varnothing 2 \mathrm{~mm}$, die am Ende der Sonde zu einem Hochdruckschlauchanschluß führt. Quer zum Dorn ist eine weitere Sackbohrung $\varnothing 2 \mathrm{~mm}$ angebracht, die die Zentralbohrung in der Mittelachse des Dorns trifft. In $45 \mathrm{~mm}$ axialem Abstand voneinander sind links und rechts von der Querbohrung zwei Ringnuten mit 2,7 $\mathrm{mm}$ Breite und 1,6 mm Tiefe eingedreht, die mit NBR-Gleitkolbendichtringen $\varnothing 6,4$ x 1,9 (12-mm-Dorn) bzw. $\varnothing 10,0$ x 2,0 (16-mm-Dorn) aus Nitrilbutyl der Shore-Härte 90 der Firma mfe bestückt wurden.

Für die Vorversuche zur Ausreißkraft wurden vom Lieferanten der Rohrbündelkomponenten jeweils zehn Testhülsen $\varnothing 23,96 \mathrm{~mm}$ und $\varnothing 32,16 \mathrm{~mm}$ mit jeweils 
zehn $150 \mathrm{~mm}$ langen Rohrproben $\varnothing 12$ und $\varnothing 16 \mathrm{~mm}$ zur Verfügung gestellt. Diese wurden bei zehn verschiedenen Sondendrücken aufgeweitet. Die Werkstoffkennwerte der Rohrproben wurden in eigenen Zugversuchen gemäß DIN EN 10091 [DIN91] ermittelt. Mit Hilfe eines mit Dehnungsmeßstreifen (DMS) in Kreuzbrückenschaltung bestückten Schraubstocks als Ausreißvorrichtung und eines Hottinger Vielstellenmeßgeräts UPM-100 konnten für jeden Aufweitdruck die tatsächlich zur Lösung des Haftverbandes von Rohr und Hülse notwendige Ausreißkraft bestimmt werden. Drei Proben $\varnothing 16 \mathrm{~mm}$ und eine Probe $\varnothing 12 \mathrm{~mm}$ wurden bei den Vorversuchen durch gerissene Dichtungsringe bzw. Materialrisse unbrauchbar. Die gemessenen und berechneten Ausreißkräfte sind in Abbildung 3-3 in Abhängigkeit vom Aufweitdruck dargestellt.

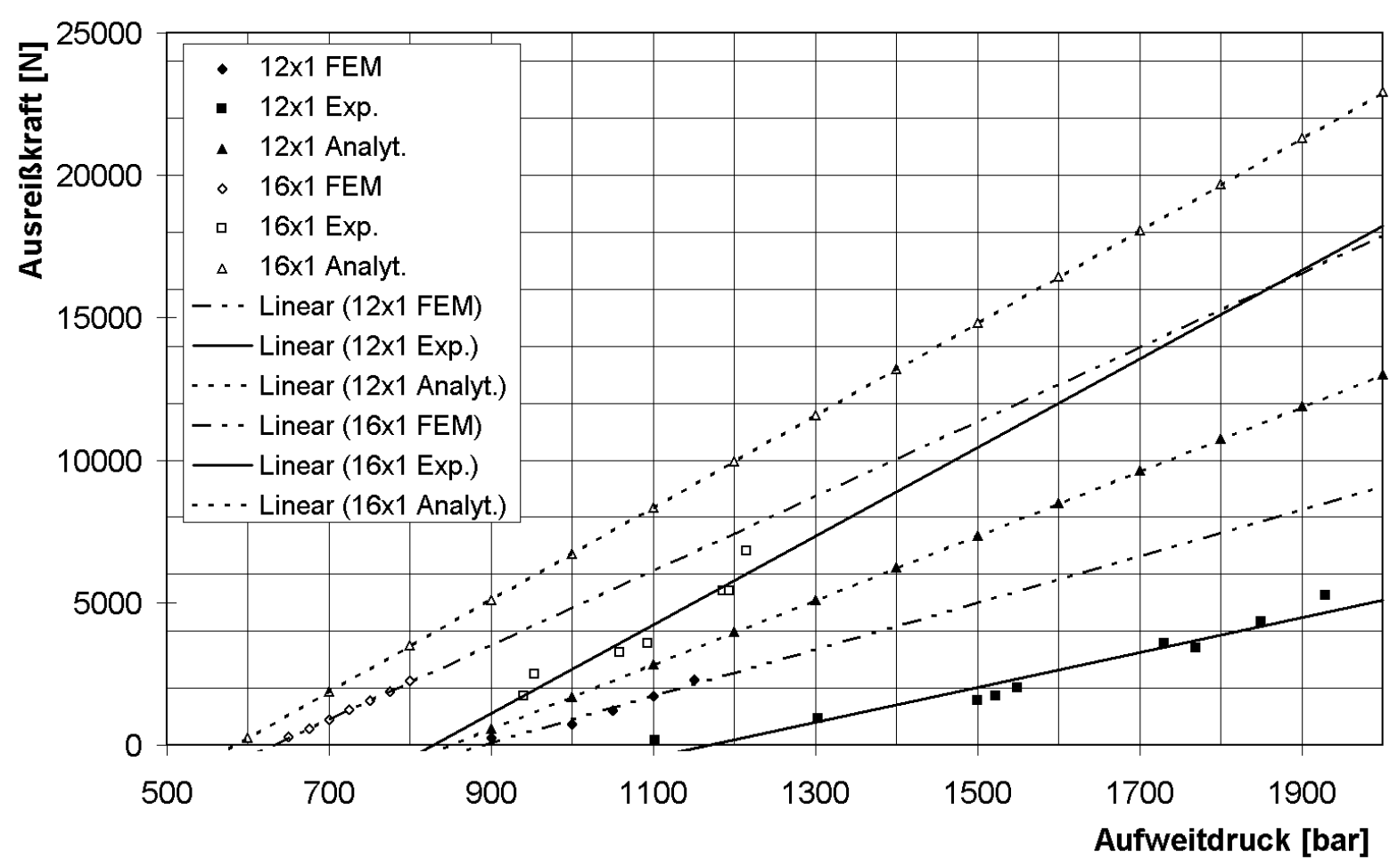

Abbildung 3-3: Rohrausreißkräfte bei analytischer und numerischer Berechnung (Reibbeiwert $=0,2$ ) im Vergleich zu experimentellen Werten.

In Tabelle 3-1 sind die notwendigen Aufweitdrücke, die nach den drei beschriebenen Verfahren ermittelt wurden, gegenübergestellt. Der nach Hölzl berechnete Aufweitdruck für das Erreichen von $3,1 \mathrm{kN}$ bzw. 4,2 kN Ausreißkraft wurde um 153 bar bzw. 460 bar zu gering berechnet. Hölzls Modell geht von idealplastischem Werkstoffverhalten aus. Wie die Spannungs-Dehnungs-Diagramme der DIN-Normproben [DIN91] des verwendeten Rohrwerkstoffs jedoch zeigten, 
verhalten sich die Rohrproben aus AlMgSi 0,5 F22 entgegen den in Hölzls Arbeit [Hö198] verwendeten Materialien nicht ideal-plastisch, sondern besitzen einen ausgeprägten Verfestigungsbereich ohne ausgeprägte Fließgrenze. Hölzl führte daher die zu gering berechneten notwendigen Aufweitdrücke auf das nicht-idealplastische Verhalten der Rohre zurück [Hö197]. Insbesondere ergibt sich aus den Messungen für die 16-mm-Rohre annahmegemäß ein rechnerischer Reibungsbeiwert von 0,2, während dieser bei den 12-mm-Rohren nur noch einen Wert von 0,11 annimmt. Offensichtlich trägt hier der Tonneneffekt wegen der dickwandigeren Ersatzhülsen weniger als bei den dünnwandigeren Ersatzhülsen der 16-mm-Proben zum Tragverhalten bei. Die analytisch bestimmten Aufweitdrücke waren von den experimentell ermittelten Werten noch etwas weiter entfernt als die FE-Ergebnisse. Hölzl führte diesen Umstand auf die Vernachlässigung von Tonneneffekt und Kontaktspitzen sowie auf die der analytischen Gleichung ebenfalls zugrundeliegende Annahme idealplastischen Werkstoffverhaltens zurück [Hö197].

\begin{tabular}{|l|rr|rr|}
\hline Aufweitdrücke [bar] & \multicolumn{2}{|c|}{$12-\mathrm{mm}-\operatorname{Rohr}(3,1 \mathrm{kN})$} & \multicolumn{2}{|c|}{$16-\mathrm{mm}-\operatorname{Rohr}(4,2 \mathrm{kN})$} \\
\hline Analytisch & 1124 & $(67,3 \%)$ & 838 & $(76,7 \%)$ \\
\hline Numerisch & 1210 & $(72,5 \%)$ & 940 & $(86,0 \%)$ \\
\hline Experimentell & 1670 & $(100,0 \%)$ & 1093 & $(100,0 \%)$ \\
\hline
\end{tabular}

Tabelle 3-1: Notwendige Aufweitdrücke zur Herstellung dauerfester RohrRohrplattenverbindungen nach drei verschiedenen Bestimmungsverfahren.

Nach den Ergebnissen der Aufweitversuche wurde der optimale Aufweitdruck für die 12-mm-Rohre auf 1700 bar und für die 16-mm-Rohre auf 1100 bar festgelegt. Der Lieferant der Rohrplatten sorgte nachweislich dafür, daß die Oberflächengüten in den Bohrungen der Proben und der Rohrplatten übereinstimmten. Um Erfahrungswerte bei der „Serienproduktion“, insbesondere im Hinblick auf den Verschleiß der Dichtringe, die nach Firmenauskunft im dynamischen Betrieb für maximal 600 bar Betriebsdruck geeignet sind, zu erlangen, wurden zunächst vier Testrohrbündel hergestellt, bei denen die Rohre nur einseitig in der Rohrplatte fixiert sind. Ein Nachweiten von einmal aufgeweiteten Rohren ist aufgrund der dann nicht mehr in ausreichendem Maße zustande kommenden Dichtung zwischen Dichtungsring und Rohrinnenseite nicht mehr möglich. 
Hätte bei den Vorversuchen ein Dichtungsring versagt, hätte das Rohr mit einem Hammer herausgeschlagen werden können. Bei den zweiseitig eingespannten Rohrbündeln wäre ein Versagen eines Dichtringes problematischer, da das entsprechende Rohr nicht hätte nachgeweitet werden können und bei bereits erfolgter erster Fixierung auch nur sehr mühsam zu entfernen gewesen wäre, wobei von einer Beschädigung der Bohrungsoberfläche auszugehen gewesen wäre.

Zur Montage wurden die Rohrplatten auf einem Aufspanntisch fixiert. Mit einer Hochdruckpumpe wurde der Spaltraum zwischen Rohr, Dichtungsringen und Aufweitdorn unter Druck gesetzt. Jeder Dichtungsring konnte bis zu 30 mal wiederverwendet werden, bevor er Risse entlang der Oberfläche aufwies und vorsorglich ausgetauscht wurde. Auf diese Art konnten die annährend tausend Aufweitungen der Testrohrbündel ohne ein einziges Versagen eines Dichtungsrings innerhalb von zwei Arbeitstagen ausgeführt werden.

Im letzten Schritt konnten die gesammelten Erfahrungswerte auf die acht zweiseitig eingespannten Rohrbündel übertragen werden. Dabei wurde zunächst eine Rohrplatte vollständig bestückt, bevor die zweite Platte begonnen wurde, und es wurde eine besondere Reihenfolge beim Aufweiten eingehalten, um eigenfrequenzverändernde axiale Verspannungen der Rohre möglichst gering zu halten. Zusätzlich mußten noch die Leitbleche eingesetzt und mit den Gewindestangen fixiert werden. Dazu wurden wiederum beide Rohrplatten und beide Leitbleche auf dem Aufspanntisch ausgerichtet.

Bei nahezu fünftausend Aufweitungen für zwölf Bündel innerhalb von drei Arbeitswochen versagte lediglich einmal ein Dichtungsring. Während aller durchgeführten Schwingungsversuche hat sich nicht eine Verbindung gelöst. Die dargelegte Verfahrensweise zur Übertragung des Fertigungsverfahrens des hydraulischen Aufweitens von Rohren zur Herstellung von Rohr-Rohrplattenverbindungen von Laborbedingungen in die „Serienproduktion“ kann daher als äußerst sicher und zuverlässig bezeichnet werden.

\subsubsection{Zielmeßgröße und verwendete Meßtechnik}

Bei der Vorgängerarbeit von Kassera [Kas97a] mußte aufgrund von mittels DMS gemessenen Verformungen im Einspannbereich der Rohre indirekt auf die am Rohr wirkenden Kräfte und Bewegungsgrößen geschlossen werden. Dabei mußte eine genaue Vorauswahl der zu vermessenden Rohre getroffen werden, da aus Platzgründen jeweils nur drei Rohre eines Bündels mit DMS bestückt 
werden konnten. Über die Bewegungsgrößen entlang der Rohrlängsachse konnten keine Aussagen getroffen werden. Dies war nur deshalb unproblematisch, da das zu validierende FSI-Programm nur zweidimensional unter Berücksichtigung ausschließlich der ersten Eigenfrequenz rechnete. Die Messung der Verformungen an nur einer ausgezeichneten Position der Rohre mittels DMS würde jedoch insbesondere dann zu Problemen mit der Deutung von Ergebnissen führen, wenn die Rohre in höheren als der ersten Eigenform schwingen bzw. sich verschiedene Schwingungsmoden überlagern.

Daher wurde das Schwingungsverhalten der Rohre in der vorliegenden Arbeit mit Hilfe von piezo-elektrischen ICP-Beschleunigungssensoren vermessen, die mit einem integrierten Ladungsverstärker ausgestattet sind. Die Stromversorgung für den Ladungsverstärker wird von der Meßkarte aus im Meßkabel mitgeführt. Die direkte Messung der Beschleunigung zum Vergleich mit den numerischen Ergebnissen des in der vorliegenden Arbeit entwickelten FSI-Codes ist gerechtfertigt, da die lokale Beschleunigung der Rohre wegen der Verwendung der Newmark-Integration [New59] eine endogene Größe des numerischen Berechnungsschemas darstellt.

Gegenüber der bei Kassera [Kas97a] verwendeten Meßmethode unter Verwendung von Dehnungsmeßstreifen (DMS) weist die Messung von Zeitreihen mit Beschleunigungssensoren eine Reihe von Vorteilen auf. So gibt es keine Einschränkungen mehr in Bezug auf die Auswahl des zu vermessenden Rohres, da ein Beschleunigungsaufnehmer leicht aus einem Rohr entfernt und in ein anderes Rohr eingesetzt werden kann. Die Beschleunigung eines Rohres kann auBerdem entlang der gesamten Rohrlängsachse vermessen werden. Insbesondere können damit alle Eigenformen der schwingenden Rohre erfaßt werden. Dies wurde notwendig, da durch die Verwendung von Leitblechen davon auszugehen war, daß auch höhere als nur erste Eigenformen angeregt würden. Darüber hinaus hat sich herausgestellt, daß sich in den Fouriertransformierten der Beschleunigungsreihen auch die feinen Kraftwirkungen von Familien ablösender Wirbel bei unterschiedlichen Volumenströmen nachweisen lassen, während diese Informationen bei der Integration der Meßreihen zu Verschiebungsgrößen weggemittelt werden. Insofern erwies sich die Auswertung der Beschleunigungsreihen als sensitiver als die Auswertung von Weg-, Geschwindigkeitsoder Dehnungsgrößen. Bei Verwendung von Beschleunigungssensoren sind daher mit geringerem Aufwand aussagekräftigere Meßergebnisse zu erwarten als bei Verwendung von DMS. Für die Befestigung der Sensoren in den Rohren 
wird eine Aufnahmevorrichtung benötigt, die im Betrieb verrutsch-, verdreh-, und rüttelsicher ist, jedoch schnell und einfach von oben gesichert und wieder gelöst werden kann. Dadurch können die Sensoren in den Rohren längsachsenverschieblich auf verschiedenen Meßhöhen ang ebracht werden.

In Abbildung 3-4 ist die hierfür entwickelte Aufnahmevorrichtung [Dra98] dargestellt. Die Sensoren sind mit Sekundenkleber verklebt und mit Lack druckwassergeschützt im Korpus der Aufnahmevorrichtung gelagert. Über eine Hohlschraube, die von oben mit Hilfe eines Bajonettwerkzeugs festgezogen und gelöst werden kann, werden zwei Keile über zwei schiefe Ebenen an der Außenseite des Korpus mit der Rohrinnenseite verspannt. In der Mittelachse der Hohlschraube verläuft das Meßkabel nach oben. Für das Gegenmoment des ersten Anziehens sorgt ein Federring, der mit geringer Kraft gegen die Innenwand der Rohre drückt und gleichzeitig ein Auseinanderfallen der beiden Halbschalen bei der Demontage verhindert.

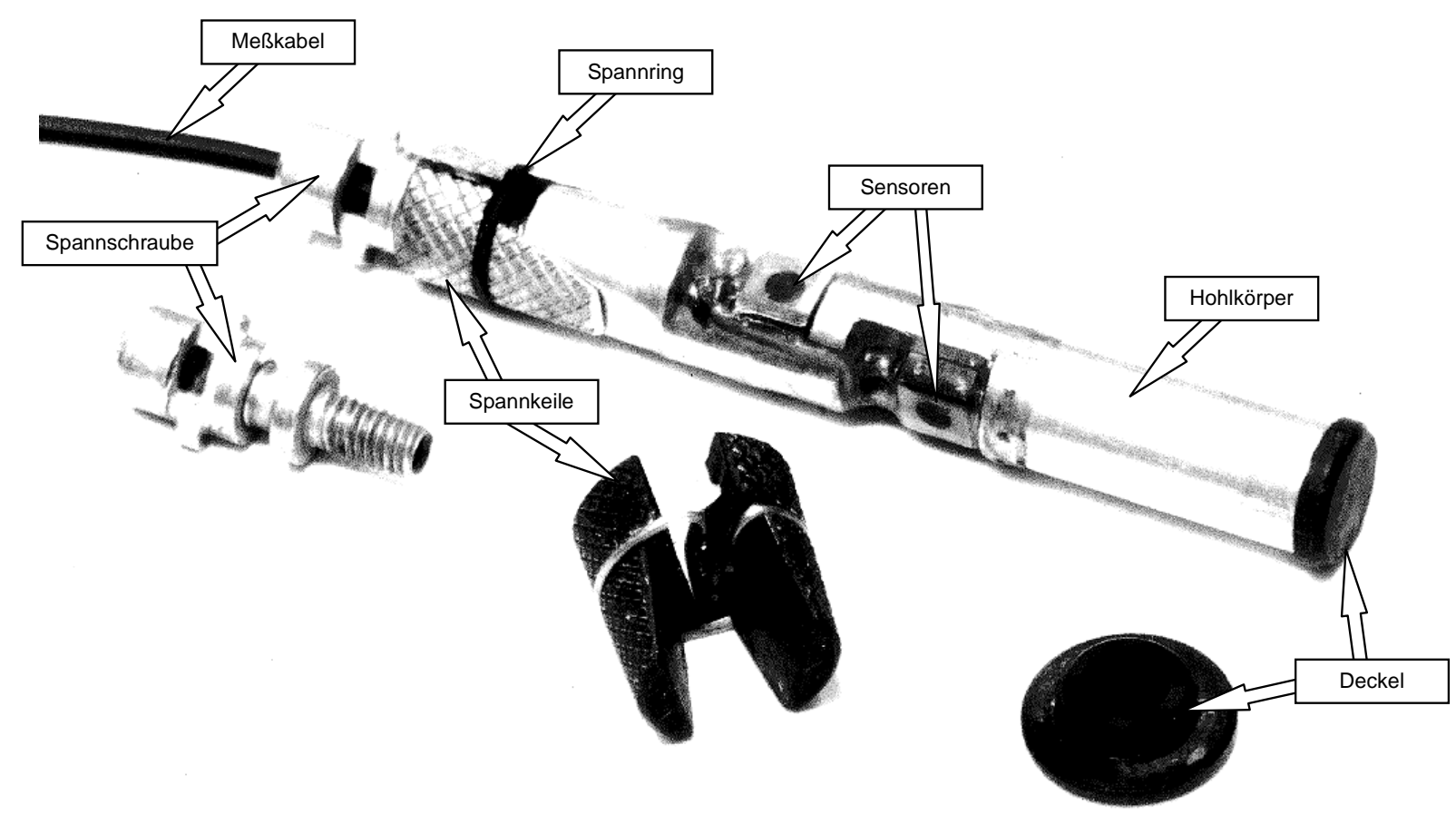

Abbildung 3-4: Aufnahmevorrichtung für Piezo-Beschleunigungssensoren.

An der Unterseite ist ein dünnwandiger Hohlzylinder angebracht, der mittels einer Preßpassung über einen Kunststoffdeckel gedichtet wird. Der Hohlkörper sorgt dafür, daß die in Leichtbauweise aus der Aluminiumlegierung AlMg 4,5 Mn gefertigte Vorrichtung im Mittel fast die Dichte von Wasser erreicht [Dra98]. Dadurch wird das Meßergebnis nicht mehr beeinflußt, da die Rohre 
innen ebenfalls mit Wasser geflutet sind. Der Deckel wirkt wie ein zweiter Lagerpunkt für die Aufnahmevorrichtung, wodurch deren Eigenfrequenz auf ca. $20 \mathrm{kHz}$ erhöht wird und die Messungen der niederfrequenteren Rohrschwingungen nicht beeinflußt werden können [Dra98]. Das Material der Aufnahmevorrichtung wurde aufgrund seiner geringen Dichte, seiner hohen Korrosionsbeständigkeit, seiner hohen Zugfestigkeit und im Hinblick auf die filigrane und dünnwandige Ausführung wegen seiner guten Zerspanbarkeit gewählt [Dra98]. Durch Austauschen der Spannkeile und der Kunststoffdeckel kann die Vorrichtung für Messungen sowohl in 12-mm-Rohren als auch in 16-mm-Rohren eingesetzt werden. Die Lage der Meßachsen zur Hauptströmungsrichtung ist durch die koordianteninvariante Auswertung der Messungen nicht von Belang, d.h., auf die Verdrehung der Sensoren im Rohr kommt es nicht an.

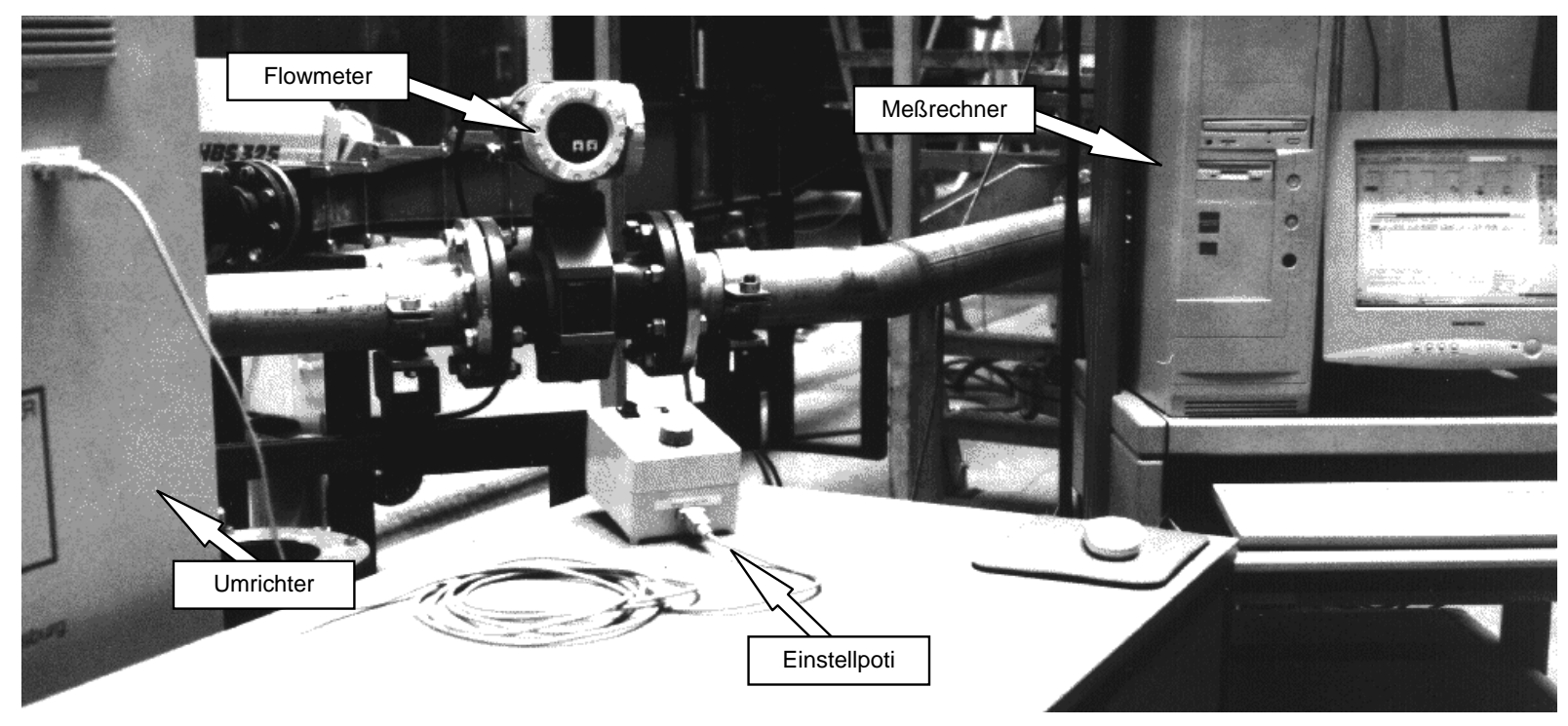

Abbildung 3-5: Meßplatz des Versuchsstandes.

Als Meßsoftware kam FlexProControl 4.0 der Firma Weisang unter Windows 95 auf einem Pentium-166-Meßrechner mit 64 MByte Arbeitsspeicher zum Einsatz. Der Meßrechner war mit zwei ICP-A/D-Meßwandlerkarten AD416SC der Firma STAC bestückt, die über jeweils vier getrennte Kanäle verfügten. Somit konnten gleichzeitig jeweils vier Rohrpositionen in vier verschiedenen Rohren vermessen werden. Pro Kanal war eine maximale Abtastrate von ca. 2,5 kHz möglich. Die Sensoren waren über BNC-Meßkabel und Lemosa-Stecker an der Rückseite des Meßrechners mit den Meßkarten verbunden. Meßkarten und Sensoren waren zur Ausschaltung von Störspannungen über das Rohrleitungsnetz 
geerdet. Die gemessenen Beschleunigungs-Zeitreihen wurden auf die rechnerinternen Festplatten mit 8 GByte Speicherkapazität zwischengespeichert, bevor sie über ein Windows-NT-Netzwerk zum Auswertungsrechner übertragen wurden. Abbildung 3-5 zeigt den Meßplatz.

\subsection{Versuchsprogramm}

\subsubsection{Variierte Parameter}

Um das Berechnungsverfahren in einem größeren Parameterfeld überprüfen zu können, wurden insgesamt acht unterschiedliche Rohrbündel zu Meßzwecken hergestellt. Die Rohrbündel unterscheiden sich in der Bohrbildanordnung (30-Grad-Teilung bzw. 90-Grad-Teilung), im Außendurchmesser der Rohre (12 $\mathrm{mm}$ bzw. $16 \mathrm{~mm}$ ) sowie in der Rohrbündellänge (1,6 m bzw. 2,1 m freie Rohrlänge). Außerdem wurde der Volumenstrom in der Meßstrecke zwischen 50 $\mathrm{m}^{3} / \mathrm{h}$ und $110 \mathrm{~m}^{3} / \mathrm{h}$ in Schritten zu je $10 \mathrm{~m}^{3} / \mathrm{h}$ variiert. Die Schwingungsversuche wurden mit Leitungswasser mit einer Dichte von $1000 \mathrm{~kg} / \mathrm{m}^{3}$ und einer Zähigkeit von 0,001 Pas durchgeführt.

Schon zu Beginn der Arbeiten fiel einer der acht Meßkanäle aus, so daß gleichzeitig je Meßvorgang Beschleunigungen in drei Meßrohren in zwei zueinander senkrecht stehenden Schwingungsrichtungen in einer festgelegten Meßtiefe aufgenommen werden konnten. Um Eigenformen messen zu können, wurde in acht äquidistanten Meßtiefen gemessen, die so gewählt waren, daß die zwei Meßtiefen auf Höhe der Leitbleche mit in den Messungen enthalten waren, sowie jeweils zwei Meßpositionen zwischen den möglichen Lagerstellen. Die gewählten Meßtiefen können Abbildung 3-6 entnommen werden.

Die Meßrohre wurden so ausgewählt, daß ihre Position im Strömungsfeld zu einer charakteristischen Beanspruchung führen mußte. So wurden insbesondere Rohre der ersten und letzten fünf Rohrreihen gewählt, die innerhalb von Fensterausschnitten positioniert sind. Die ausgewählten Positionen liegen außerdem meist in der Nähe der Zuström- bzw. Abströmstutzen, wo infolge erhöhter Strömungsgeschwindigkeit mit erhöhter Belastung zu rechnen ist. Zweifach gestütze Rohre erfahren eine geringere Belastung. Hier wurden Rohre gewählt, die in der Nähe der Kante der Leitbleche liegen, da hier die relativ für zweifach gestützte Rohre höchste Belastung zu erwarten ist. Außerdem wurden noch Randrohre gewählt, um den Einfluß der Umströmung der Rohrbündel am äußersten Rand der Berohrung erfassen zu können. 


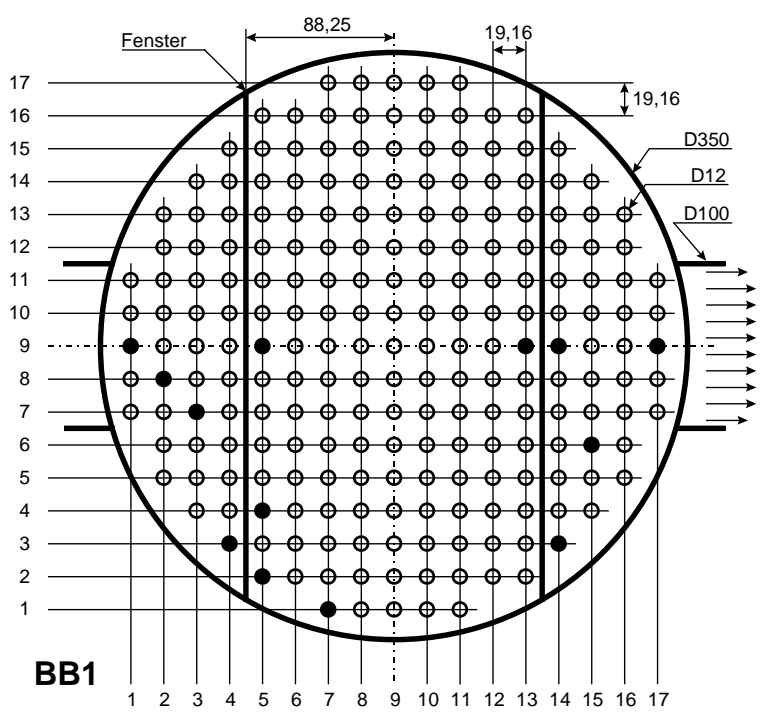

Bohrbild 1

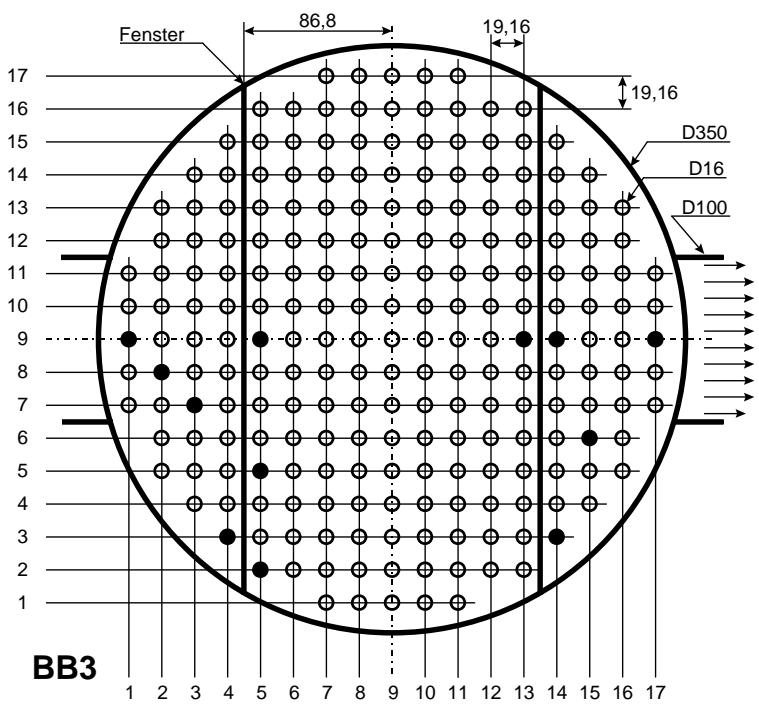

Bohrbild 3

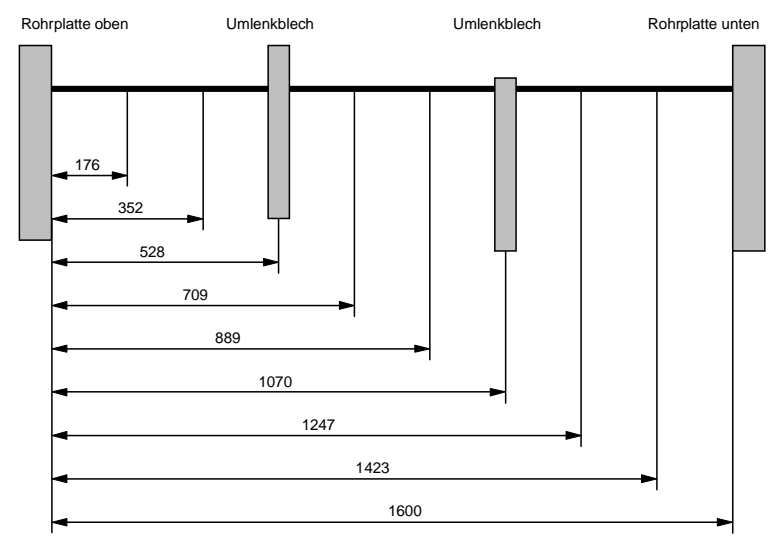

Rohrbündellänge 1,6 m

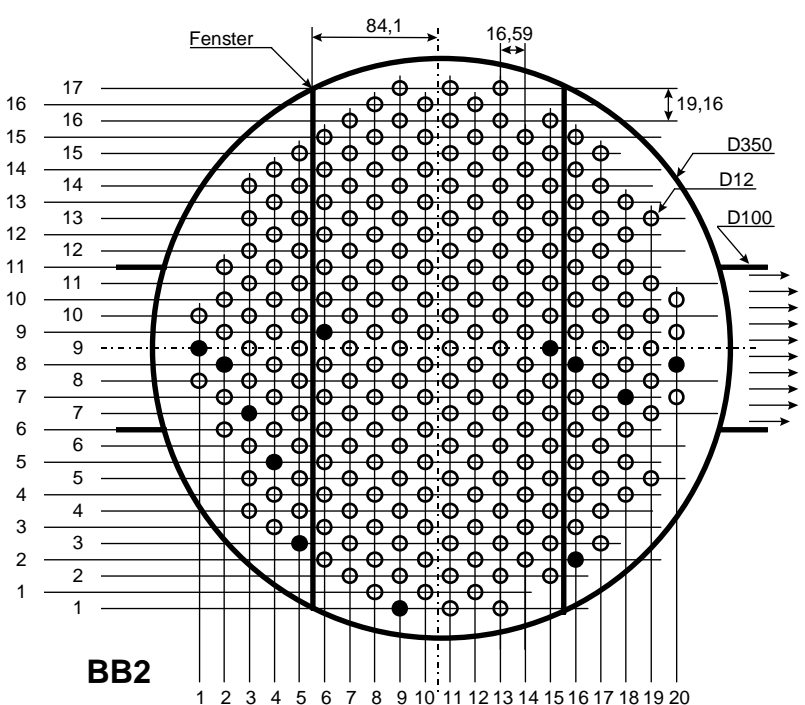

Bohrbild 2

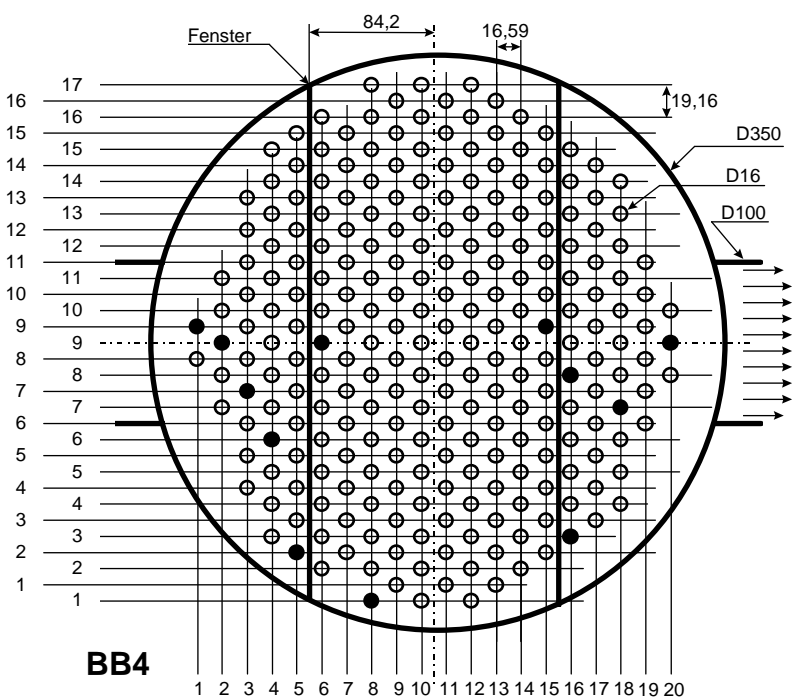

Bohrbild 4

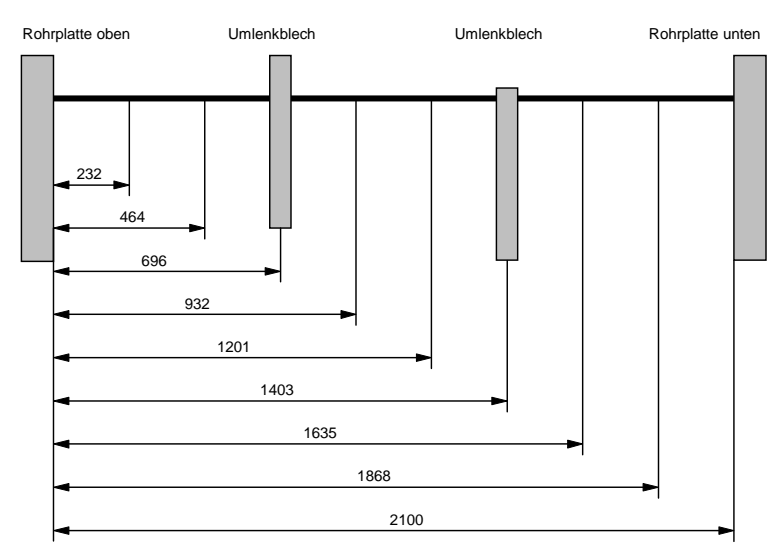

Rohrbündellänge 2,1 m

Abbildung 3-6: Rohrbündelgeometrien. 


\begin{tabular}{|c|c|c|c|c|}
\hline \multirow{2}{*}{ Rohrbündel } & \multicolumn{4}{|c|}{ Vermessene Rohrpositionen } \\
\hline \multirow{3}{*}{ BB1-L170 } & $01-09$ & $05-02$ & $04-03$ & $03-07$ \\
& $02-08$ & $05-09$ & $14-03$ & $05-04$ \\
& $17-09$ & $13-09$ & $14-09$ & $15-06$ \\
\hline \multirow{3}{*}{ BB2-L170 } & $01-09$ & $06-09$ & $05-03$ & $(03-07)$ \\
& $02-08$ & $09-01$ & $16-02$ & $(04-05)$ \\
& $20-08$ & $15-09$ & $(16-08)$ & $18-07$ \\
\hline \multirow{3}{*}{ BB3-L170 } & $01-09$ & $05-02$ & $(04-03)$ & $03-07$ \\
& $02-08$ & $05-09$ & $14-03$ & $05-05$ \\
& $17-09$ & $(13-09)$ & $14-09$ & $15-06$ \\
\hline & $(01-09)$ & $(06-09)$ & $(05-02)$ & $(03-07)$ \\
& $02-09$ & $08-01$ & $(16-03)$ & $04-06$ \\
BB4-L170 & $20-09$ & $15-09$ & $16-08$ & $18-07$ \\
\hline & $01-09$ & $05-09$ & $04-03$ & $03-07$ \\
& $02-08$ & $07-01$ & $14-03$ & $05-04$ \\
& $17-09$ & $13-09$ & $14-09$ & $15-06$ \\
\hline \multirow{3}{*}{ BB2-L220 } & $(01-09)$ & $06-09$ & $05-02$ & $03-07$ \\
& $02-08$ & $09-01$ & $16-03$ & $04-05$ \\
& $20-09$ & $15-09$ & $16-08$ & $18-07$ \\
\hline \multirow{3}{*}{ BB3-L220 } & $01-09$ & $05-02$ & $04-03$ & $03-07$ \\
& $02-08$ & $05-09$ & $14-03$ & $05-05$ \\
& $17-09$ & $13-09$ & $14-09$ & $15-06$ \\
\hline & $01-09$ & $06-09$ & $05-02$ & $03-07$ \\
& $02-08$ & $08-01$ & $16-03$ & $04-06$ \\
& $20-09$ & $15-09$ & $16-08$ & $18-07$ \\
\hline
\end{tabular}

Tabelle 3-2: Vermessene Rohrpositionen.

In Abbildung 3-6 sind außer den gewählten Meßtiefen auch die verwendeten Bohrbilder 1 bis 4 dargestellt. Die Rohre in Bohrbild 1 und 2 haben jeweils einen Durchmesser von $12 \mathrm{~mm}$, die in Bohrbild 3 und 4 einen Durchmesser von $16 \mathrm{~mm}$. Auch die Kanten der Leitblechfenster sind dargestellt.

Tabelle 3-2 enthält alle vermessenen Rohrpositionen. Messungen, die aufgrund von Defekten an den Meßaufnehmern oder den Meßkarten unbrauchbar wurden, sind eingeklammert markiert. Insgesamt wurden bei acht Rohrbündeln jeweils 
$12 \mathrm{Meßpositionen} \mathrm{in} \mathrm{acht} \mathrm{verschiedenen} \mathrm{Meßtiefen} \mathrm{bei} \mathrm{sieben} \mathrm{unterschiedlichen}$ Volumenströmen vermessen. Es ergab sich dabei ca. 6 GByte Datenmaterial.

\section{$\underline{3.2 .2 \text { Versuchsablauf }}$}

Zur Versuchsdurchführung wird zunächst der Behälter so umgebaut, daß er die passende Höhe für die vorgesehene Rohrbündellänge aufweist. Durch die modulare Konstruktion ist es möglich, durch Austausch des Mittelteils (19) zwei verschiedene Rohrbündellängen zu verwenden. Dann wird mit Hilfe eines Hallenkrans das gewünschte Rohrbündel von oben in den Behälter eingesetzt und von unten an den Kunststoffring angeschraubt. Am oberen Ende wird das Bündel in radialer Richtung durch vier Gleitrollen aus Kunststoff fixiert, so daß Längenausdehnung des Bündels ermöglicht wird, eine radiale Bewegung der oberen Rohrplatte jedoch verhindert wird.

Nach Einsetzen des Bündels wird der Behälter unten durch Anschrauben eines Deckels über einen Flansch verschlossen. Von oben werden mit Hilfe von Spezialwerkzeugen die Sensoraufnehmer in die ausgewählten Meßrohre eingesetzt und durch Schraubung fixiert. Die Meßkabellänge wird dabei nur einmal auf die unterste vorgesehene Meßposition angepaßt und dann mittels einer Knetmassendichtung in einer Verschraubung am Schnellverschlußdeckel (21) abgedichtet. Bei höheren Meßpositionen wird die überflüssige Kabellänge mit Kabelbindern aufgeschossen und lagert während des Versuchs oberhalb der oberen Rohrplatte im Versuchsbehälter. Auf diese Weise muß die Kabelführung nicht für jede Meßposition neu abgedichtet werden. Danach kann der obere Schnellverschlußdeckel (21) geschlossen und der Meßbehälter, soweit durch frühere Meßreihen noch nicht geschehen, durch Öffnen des Absperrschiebers (13) geflutet werden. Dadurch fließt das in den Ausgleichsbehälter (1) eingefüllte Leitungswasser in die Versuchsstrecke. Abbildung 3-7 zeigt den Schnellverschlußdeckel (21) zusammen mit der Meßkabelführung.

Um die Versuche bei luftblasenfreiem Behälter durchführen zu können, muß der geschlossene Kreislauf zunächst einige Minuten bei etwa $90 \mathrm{~m} / \mathrm{h}$ Volumenstrom betrieben werden. Dadurch wird im System verbliebene Luft ausgespült. Sie entweicht über den oben offenen Ausgleichsbehälter (1) in die Umgebung. Steigen im Ausgleichsbehälter (1) keine Luftblasen mehr auf, kann der obere Entlüftungsschlauch über ein Stellventil geflutet werden. Das Entlüftungsventil I wird so eingestellt, daß über den Entlüftungsschlauch immer nur ein kleines Rinnsal in den Ausgleichsbehälter zurückfließt. So ist gewährleistet, daß der 
Versuch immer luftblasenfrei abläuft und am Austritt der Meßstrecke derselbe Massenstrom wirkt wie am Eintritt.

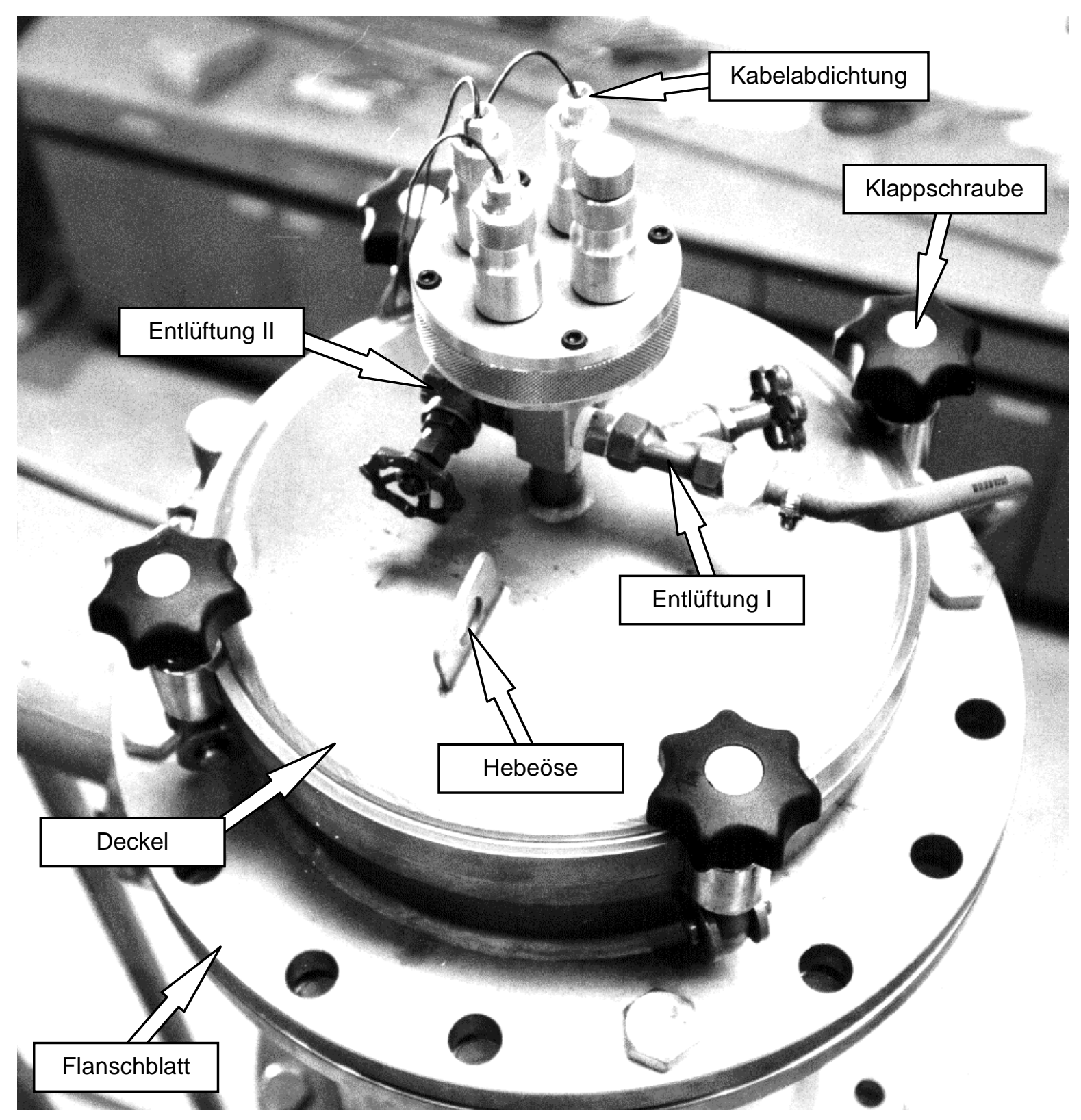

Abbildung 3-7: Schnellverschlußdeckel der Meßstrecke mit Kabelführung.

Nachdem die Luft dynamisch aus dem System herausgedrückt wurde, kann auch bei verringertem Volumenstrom blasenfrei gemessen werden. Hierzu wird über das Stellpotentiometer des Frequenzumrichters die Drehzahl des Elektromotors (2) so eingestellt, daß sich am Volumenstrommeßgerät (15) der passende Volumenstrom einstellt. Bei diesen stationären Bedingungen werden dann nacheinander drei Messungen von je 2 s Dauer bei einer zeitlichen Auflösung von 0,000488 s durchgeführt. Dies entspricht einer Abtastung von $2 \mathrm{kHz}$ je Meßka- 
nal. Die Auswertungsresultate der drei zueinander gehörenden Meßreihen werden später gemittelt, um zufällige Schwankungen auszugleichen. Dieses Verfahren hatte sich bereits bei Kassera [Kas97a] bewährt.

Nach Ende einer Versuchsreihe wird die Drehzahl der Pumpe auf Null reduziert. Um den Schnellverschluß (21) öffnen zu können, wird der sich durch das absinkende Wasser einstellende Unterdruck im Dom des Schnellverschlusses (21) durch das Öffnen des Entlüftungsventils II am Schnellverschluß abgebaut. Danach kann eine neue Meßreihe vorbereitet werden.

\subsection{Auswertung}

\subsubsection{RMS-Werte in Abhängigkeit der Rohrlängskoordinate}

Als RMS-Wert (root mean square) einer Beschleunigungsmeßreihe $A_{i}$ wird der Wert

$$
A_{R M S}=\sqrt{\frac{1}{n-1} \sum_{i=1}^{n}\left(\bar{A}-A_{i}\right)^{2}}
$$

verwendet. Der RMS-Wert ist ein Maß für den mittleren Ausschlag bzw. die Standardabweichung einer Reihe von Werten. Dabei ist

$$
\bar{A}=\frac{1}{n} \sum_{i=1}^{n} A_{i}
$$

der Mittelwert bzw. der Erwartungswert der Meßreihe und $n$ ist die Anzahl der Meßpunkte der Meßreihe.

Der RMS-Wert wird zur Beurteilung der Schwingbelastung eines Rohres verwendet, der Mittelwert stellt ein Maß für die Grundlast dar. Um die Schwierigkeit der Feststellung der genauen Winkelstellung des Meßsensors in den jeweiligen Rohren umgehen zu können, wurde für die vorliegende Arbeit als koordinateninvariante Meßgröße der RMS-Wert des Betrages des Beschleunigungsvektors gebildet. Der Betrag des Beschleunigungsvektors ergibt sich aus der quadratischen Addition der beiden zueinander rechtwinkligen Meßgrößen eines Sensorpaares.

Trägt man nun die RMS-Werte über der Rohrlängsachsenkoordinate auf, so erhält man für jedes vermessene Rohr eine charakteristische Darstellung, die Auskunft darüber gibt, in welchen Bereichen ein Rohr hohen oder geringen Schwingbelastungen ausgesetzt ist. Abbildung 3-8 zeigt ein Beispiel dafür. Um 
zufällige Fehler in den Messungen auszugleichen, wurde, dem Vorgehen von Kassera folgend [Kas97a], von jeweils drei Messungen unter gleichen Randbedingungen der Mittelwert der drei RMS-Werte als Vergleichsgröße verwendet.

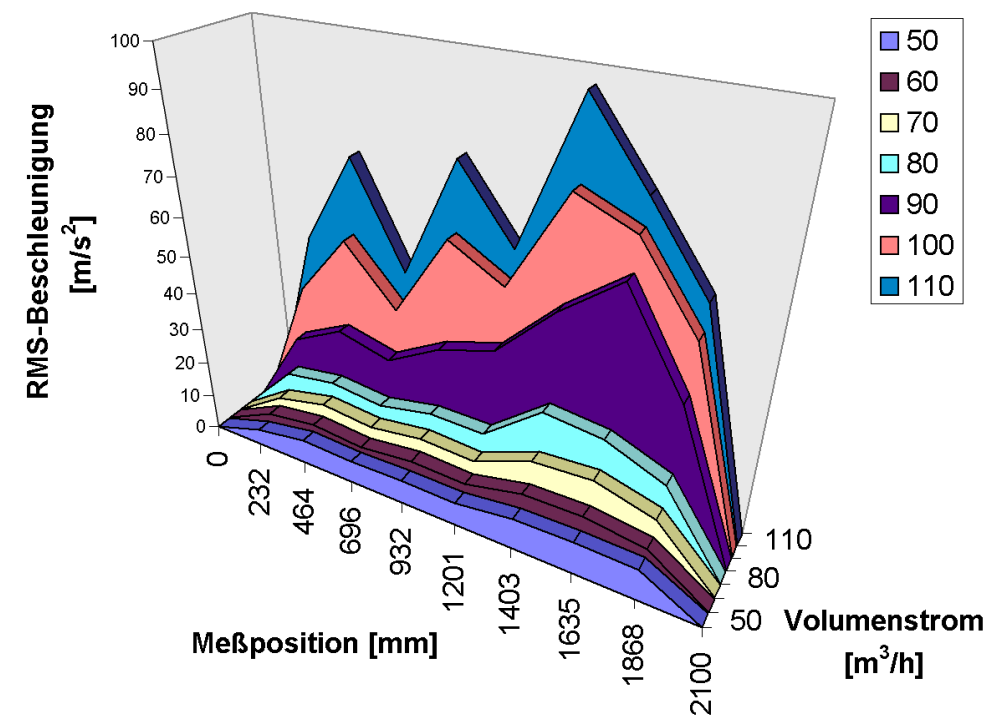

Abbildung 3-8: Verteilung der gemessenen RMS-Beschleunigung am Beispiel von Rohr BB4-L220-R0406.

\subsubsection{Fourier-Transformation}

Um analysieren zu können, welche Frequenzanteile in einer Meßreihe enthalten sind, bedient man sich der diskreten Fouriertransformation [Nat92]. In komplexer Schreibweise ergibt sich die Fouriertransformierte zu [Wol97]

$$
B_{i}=\sum_{r=1}^{n} A_{r} e^{2 \pi \sqrt{-1}(r-1)(i-1) / n}
$$

Dabei wird die zur Frequenz $0 \mathrm{~Hz}$ gehörende Transformierte der Position $1 \mathrm{zu}$ geordnet [Wo197]. Die den Koeffizienten $i$ zugeordneten Frequenzen $f_{i}$ ergeben sich damit aus dem Kehrwert der Meßzeit $\Delta T$ und der Position $i$ der transformierten Reihe zu [Nat92]

$$
f_{i}=\frac{i-1}{\Delta T}
$$

Die maximal erreichbare Frequenzauflösung ist damit $1 / \Delta T$. $\mathrm{Zu}$ beachten ist bei der Auswertung der Reihe der Aliasing-Effekt. Dadurch sind die im Frequenzraum dargestellten Koeffizienten der Fourierreihe zur Hälfte der größten darge- 
stellten Frequenz spiegelsymmetrisch [Nat92]. Zur Identifizierung der Eigenfrequenzen wurde von jeder Fouriertransformierten einer Meßreihe der Betrag der komplexen Koeffizienten $B_{i}$ verwendet. Um wiederum zu einer koordinateninvarianten Auswertung zu gelangen, wurden die jeweiligen Koeffizienten für die beiden zueinander senkrechten Beschleunigungskomponenten quadratisch addiert. Um zufällige Meßfehler zu eliminieren, wurden wiederum jeweils drei unter gleichen Bedingungen aufgenommene Fourierreihen gemittelt.

\subsubsection{Rohrcharakteristische Fouriertransformierte}

Es wurden bei allen Meßrohren Meßreihen in acht unterschiedlichen Meßtiefen aufgenommen. Um die Menge der sich ergebenden Meßdaten zu kondensieren, wurde für jedes Rohr eine rohrcharakteristische Fouriertransformation gebildet. Hierzu wurden die für jede Meßtiefe vorhandenen Fouriertransformationen mit den für die jeweilige Meßreihe berechneten RMS-Werten der Beschleunigung gewichtet. Diesem Vorgehen liegt der Gedanke zugrunde, daß jede Fourierreihe ja angibt, welcher Energieanteil der dynamischen Schwingbewegung des Rohres welchem Frequenzband zukommt. Dort, wo eine höhere RMS-Amplitude auftritt, wird zudem ein höherer Energieanteil vorzufinden sein. Durch die Wichtung mit den RMS-Werten ergibt sich somit eine Darstellung, die für das gesamte Rohr angibt, welcher Energieanteil welchem Frequenzband zuzuordenen ist. Abbildung 3-9 zeigt ein Beispiel.

Um nachzuweisen, daß bei diesem Vorgehen keine Informationen über relevante Frequenzanteile verloren gehen, wurden bei einer zufälligen Stichprobe von $25 \%$ aller Meßrohre die charakteristischen Fourierdiagramme für alle gemessenen Volumenströme mit den jeweiligen Diagrammen der Messungen für alle Meßtiefen verglichen. Dabei ergab sich, daß alle Peaks im Frequenzraum, die bei den Einzelmessungen aufgetreten waren, auch in der charakteristischen Fouriertransformierten wiederzufinden waren. Somit ist gesichert, daß bei der dargestellten Auswertemethode keinerlei relevante Informationen verloren gehen. Gleichzeitig verringert sich bei acht Meßtiefen in jedem Rohr die Menge der auszuwertenden Größen um einen Faktor acht. Damit existiert mit der RMSDarstellung und der charakteristischen Fouriertransformierten für jedes vermessene Rohr eine charakteristische Beschreibung seines Schwingungsve rhaltens. In Abbildung 3-9 sind bereits die analytisch berechneten ersten sechs Eigenfrequenzen für die relevanten Lagerungsfälle mit eingezeichnet. Die Berechnung der Eigenfrequenzen der Rohre wird im folgenden Abschnitt dargestellt. 


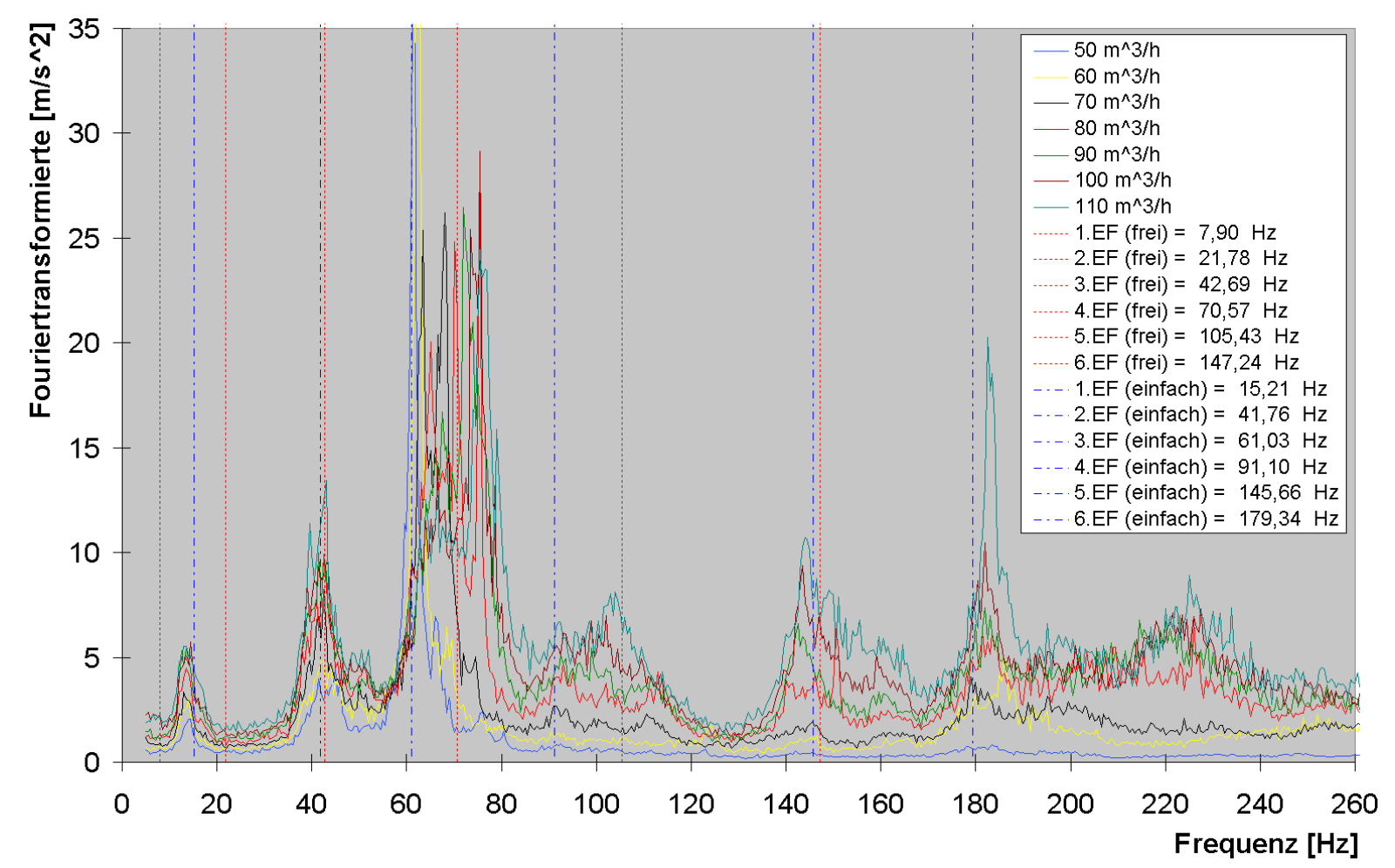

Abbildung 3-9: Charakteristische Fouriertransformation am Beispiel des Rohres BB1-L220-R0109.

\subsubsection{Analytische Identifikation der Schwingungsmoden}

Um numerische Berechnungsergebnisse und experimentelle Resultate hinsichtlich des auftretenden Schwingungsspektrums miteinander vergleichen zu können, ist es notwendig, die einzelnen in der Spektralanalyse der schwingenden Rohrbündel enthaltenen Frequenzpeaks identifizieren zu können. Für die vorliegende Arbeit wurden dazu die Eigenfrequenzen der untersuchten Rohre auf analytischem Wege mit der in [Fis00b] dargestellten Methode berechnet.

Ausgangspunkt ist die Biegeschwingungsdifferentialgleichung (2-61). Um die zugehörigen Eigenfrequenzwerte berechnen zu können, werden die Lasten und die geschwindigkeitsproportionale Dämpfung zu Null gesetzt. Die Kraft $F$ verbleibt, da es möglich ist, daß sich durch Eigenspannungen eigenfrequenzverschiebende Axiallasten ergeben. Im realen Wärmetauscher sind insbesondere Temperaturlasten eine wichtige Quelle für Axiallasten. $\mathrm{Zu}$ lösen bleibt damit die partielle Differentialgleichung [Pfe92, Sza56]

$$
m \frac{\partial^{2} w}{\partial t^{2}}+E I \frac{\partial^{4} w}{\partial x^{4}}-F \frac{\partial^{2} w}{\partial x^{2}}=0
$$


Das Flächenträgheitsmoment für ein zylindrisches Rohr ist dabei

$$
I=\frac{\pi}{64}\left(D^{4}-d^{4}\right)
$$

Die Berechnung der mitschwingenden Masse pro Länge erfolgt dabei nach der Formel [TEM82, VDI97]

$$
m=\frac{\pi}{4}\left(D^{2}-d^{2}\right) \rho_{R}+\frac{\pi}{4} d^{2} \rho+\frac{\pi}{4} D^{2} \rho k
$$

Der Zusatzfaktor $k$ berücksichtigt dabei den Einfluß der umliegenden Rohre im Wärmetauscher auf die hydrodynamische Zusatzmasse des betrachteten Wärmetauscherrohrs. Er hängt vom Teilungsverhältnis $\tau=T / D$ ab und ist in Diagrammform aus dem TEMA-Standard [TEM82] oder dem VDI-Wärmeatlas [VDI97] zu entnehmen. Eine Nährungsformel, die von ihren Ergebnissen zwischen TEMA und den beiden im VDI-Wärmeatlas angegebenen Diagrammen liegt, ist

$$
k(\tau)=12,68 \tau^{4}-87,57 \tau^{3}+227,48 \tau^{2}-264,78 \tau+118,75
$$

Die Differentialgleichung kann durch einen Separationsansatz [Pfe92, Sza56]

$$
w(x, t)=\sum_{i=1}^{\infty} w_{i}(x) \cdot q_{i}(t)
$$

getrennt werden. Es ergeben sich die Differentialgleichungen [Fis00b]

$$
\begin{aligned}
\frac{\partial^{4} w_{i}}{\partial x^{4}}-\hat{\varphi} \frac{\partial^{2} w_{i}}{\partial x^{2}}-\hat{\beta}_{i}^{4} w_{i}=0 \\
\ddot{q}_{i}+\hat{\omega}_{i}^{2} q_{i}=0
\end{aligned}
$$

mit den Eigenwerten [Pfe92, Sza56]

$$
\hat{\beta}_{i}^{4}=\frac{m \hat{\omega}_{i}^{2}}{E I} \quad \hat{\omega}_{i}=2 \pi f_{i}
$$

und dem Axiallastverhältnis [Fis00b]

$$
\hat{\varphi}=\frac{F}{E I}
$$

Zur Ermittlung der Eigenfrequenzen wird nur noch die obere Differentialgleichung (3-13a) benötigt. Sie ist homogen, und man erhält durch Aufstellen des charakteristischen Polynoms die allgemeine Lösung [Bro87] 


$$
w_{i}(x)=c_{1} \cosh \hat{\beta}_{i 1} x+c_{2} \sinh \hat{\beta}_{i 1} x+c_{3} \cos \hat{\beta}_{i 2} x+c_{4} \sin \hat{\beta}_{i 2} x
$$

mit den Lösungen des charakteristischen Polynoms [Fis00b]

$$
\begin{aligned}
& \hat{\beta}_{i 1}=\sqrt{\sqrt{\hat{\beta}_{i}^{4}+\hat{\varphi}^{2}}-\hat{\varphi}} \\
& \hat{\beta}_{i 2}=\sqrt{\sqrt{\hat{\beta}_{i}^{4}+\hat{\varphi}^{2}}+\hat{\varphi}}
\end{aligned}
$$

Zur Darstellung der speziellen Lösung müssen die freien Konstanten $c_{1}$ bis $c_{4}$ aus (3-16) bestimmt werden. Die Konstanten sind dabei von der Axialkraft $F$ abhängig. Als Sonderfall wird zunächst $F=0$ betrachtet. Damit wird $\hat{\beta}_{i 1}=\hat{\beta}_{i 2}=\hat{\beta}_{i}$. Außerdem müssen für die vorliegende Arbeit drei Lagerungsfälle unterschieden werden:

1. Das Rohr liegt an keinem Leitblech an. Es müssen die Eigenfrequenzen des frei schwingenden Rohrs berechnet werden.

2. Das Rohr ist in einem Fensterausschnitt positioniert und kann daher höchstens einfach bei $1 / 3 L_{0}$ gelagert sein. Es können je nach Anströmgeschwindigkeit und Lagerspaltgröße die Eigenfrequenzen des frei schwingenden Rohrs oder diejenigen des einfach gelagerten Rohrs angeregt werden.

3. Das Rohr liegt nicht in einem Fensterausschnitt. Es kann je nach Anströmgeschwindigkeit und Lagerspaltgröße frei schwingen, in einem Lagerblech gestützt werden oder in beiden. Daher können die Eigenfrequenzen des freien, des einfach bei $1 / 3 L_{0}$ oder ${ }^{2} / 3 L_{0}$ gelagerten oder des doppelt bei $1 / 3 L_{0}$ und $2 / 3 L_{0}$ gelagerten Rohrs angeregt werden.

Als Einspannbedingung wird eine Fest-Fest-Lagerung angenommen. Aufgrund der verwendeten dicken Rohrplatten ist dies gerechtfertigt. In der vorliegenden Arbeit sind Eigenfrequenzen bis zur sechsten Ordnung relevant. Wie man sieht, müssen dann in der Auswertung bis zu 18 Eigenfrequenzen je Rohr berücksichtigt werden.

Für das frei schwingende Rohr sind demnach die Randbedingungen [Pfe92, Sza56]

$$
\begin{aligned}
w(x=0) & =0 \\
w(x=L) & =0 \\
w^{\prime}(x=0) & =0 \\
w^{\prime}(x=L) & =0
\end{aligned}
$$


mit $L=L_{0} \mathrm{zu}$ berücksichtigen. Es ergibt sich ein lineares homogenes Gleichungssystem für die vier gesuchten Konstanten. Für eine nichttriviale Lösung muß die Determinante des Systems Null sein. Für die Eigenfrequenzen ergeben sich damit unendlich viele Lösungen [Pfe92, Sza56]

$$
f_{i}=\frac{\beta_{i}^{2}}{2 \pi L^{2}} \sqrt{\frac{E I}{m}}
$$

Im Falle der einfachen Stützung bei $L=1 / 3 L_{0}$ wird das Berechnungsgebiet in zwei Teilgebiete mit zwei allgemeinen Lösungsfunktionen $w^{1}$ und $w^{2}$ unterteilt. Die Stützpunkte im Leitblech werden wie gelenkige Lagerungen behandelt. Der Nullpunkt für die beiden Lösungsfunktionen ist jeweils der linke Lagerungspunkt der beiden Gebiete. Die Eigenwerte sind für beide Ansatzfunktionen identisch. Es handelt sich um ein und denselben Körper, deshalb müssen sich in beiden Abschnitten auch die gleichen Eigenfrequenzen ergeben. Die acht zu berücksichtigenden Randbedingungen sind demnach [Fis00b, Sto00]

$$
\begin{aligned}
w^{1}\left(x_{1}=0\right) & =0 \\
w^{2}\left(x_{2}=2 L\right) & =0 \\
w^{\prime}\left(x_{1}=0\right) & =0 \\
w^{2}\left(x_{2}=2 L\right) & =0 \\
w^{1}\left(x_{1}=L\right) & =w^{2}\left(x_{2}=0\right) \\
w^{\prime}\left(x_{1}=L\right) & =w^{2}\left(x_{2}=0\right) \\
w^{1 \prime}\left(x_{1}=L\right) & =w^{2}\left(x_{2}=0\right) \\
w^{2}\left(x_{2}=0\right) & =0
\end{aligned}
$$

Im Falle der zweifachen Stützung ergeben sich mit $L=1 / 3 L_{0}$ bei wiederum identischen Eigenwerten für die drei Abschnitte entsprechend die zwölf Randbedingungen [Sto00] 


$$
\begin{gathered}
w^{1}\left(x_{1}=0\right)=0 \\
w^{2}\left(x_{2}=0\right)=0 \\
w^{3}\left(x_{3}=0\right)=0 \\
w^{3}\left(x_{3}=L\right)=0 \\
w^{\prime}\left(x_{1}=0\right)=0 \\
w^{3}\left(x_{3}=L\right)=0 \\
w^{1}\left(x_{1}=L\right)=w^{2}\left(x_{2}=0\right) \\
w^{\prime}\left(x_{1}=L\right)=w^{2}\left(x_{2}=0\right) \\
w^{\prime \prime}\left(x_{1}=L\right)=w^{2}\left(x_{2}=0\right) \\
w^{2}\left(x_{2}=L\right)=w^{3}\left(x_{3}=0\right) \\
w^{\prime}\left(x_{2}=L\right)=w^{3}\left(x_{3}=0\right) \\
w^{\prime \prime}\left(x_{2}=L\right)=w^{3 \prime}\left(x_{3}=0\right)
\end{gathered}
$$

Es ergeben sich auch in diesen beiden Fällen lineare homogene Gleichungssysteme für die acht bzw. zwölf gesuchten Konstanten. Die Eigenfrequenzen berechnen sich nach derselben Formel, jedoch ergeben sich unterschiedliche Eigenwerte $\beta_{i}$. Die Eigenwerte für alle drei Lagerungsarten sind in Tabelle 3-3 bis Tabelle 3-5 aufgelistet.

Bei zusätzlicher Wirkung von Axiallast können die Eigenfrequenzen nur noch durch numerische Auswertung der Eigenwertgleichung berechnet werden [Fis00b]. Es kann aber in Anlehnung an analytische Formeln für die erste Eigenfrequenz von Rohren unter Axiallast [VDI97] eine analytische Nährungsformel angegeben werden. Danach berechnen sich die Rohreigenfrequenzen nach der Formel [Fis00b]

$$
f_{i}=\frac{\beta_{i}^{2}}{2 \pi L^{2}} \sqrt{\frac{E I}{m}}\left(1+\frac{\hat{\varphi} L^{2}}{\varphi_{k r i t, i}}\right)^{2 \alpha_{i}}
$$

mit dem kritischen Axiallastverhältnis [Fis00b]

$$
\varphi_{\text {krit }, i}=\psi_{i}^{2}
$$




\begin{tabular}{|c|c|c|c|r|r|r|}
\hline$i$ & 1 & \multicolumn{1}{c|}{2} & \multicolumn{1}{c|}{3} & \multicolumn{1}{c|}{4} & \multicolumn{1}{c|}{5} & \multicolumn{1}{c|}{6} \\
\hline$\beta_{i}$ & 4,730 & 7,853 & 10,995 & 14,137 & 17,279 & 20,420 \\
\hline$\varphi_{k r i t, i}$ & 19,739 & 40,381 & 78,957 & 119,359 & 177,653 & 237,800 \\
\hline$\alpha_{i}$ & 0,247 & 0,245 & 0,268 & 0,255 & 0,263 & 0,258 \\
\hline
\end{tabular}

Tabelle 3-3: Lagerungsfall „freies Rohr“.

\begin{tabular}{|c|c|r|r|r|r|r|}
\hline$i$ & 1 & 2 & 3 & \multicolumn{1}{c|}{4} & \multicolumn{1}{c|}{5} & \multicolumn{1}{c|}{6} \\
\hline$\beta_{i}$ & 2,188 & 3,625 & 4,382 & 5,354 & 6,770 & 7,512 \\
\hline$\varphi_{k r i t i}$ & 3,790 & 7,900 & 13,246 & 19,738 & 26,412 & 33,817 \\
\hline$\alpha_{i}$ & 0,249 & 0,249 & 0,229 & 0,282 & 0,261 & 0,243 \\
\hline
\end{tabular}

Tabelle 3-4: Lagerungsfall „Rohr bei ${ }^{1}{ }_{3} L_{0}$ gelagert“.

\begin{tabular}{|c|c|r|r|r|r|r|}
\hline$i$ & 1 & \multicolumn{1}{c|}{2} & \multicolumn{1}{c|}{3} & \multicolumn{1}{c|}{4} & \multicolumn{1}{c|}{5} & \multicolumn{1}{c|}{6} \\
\hline$\beta_{i}$ & 3,556 & 4,298 & 4,730 & 6,708 & 7,429 & 7,850 \\
\hline$\varphi_{k r i t i}$ & 7,438 & 13,189 & 19,738 & 26,416 & 33,514 & 40,383 \\
\hline$\alpha_{i}$ & 0,250 & 0,239 & 0,243 & 0,266 & 0,248 & 0,245 \\
\hline
\end{tabular}

Tabelle 3-5: Lagerungsfall „Rohr bei ${ }^{1}{ }_{3} L_{0}$ und ${ }^{2}{ }_{3} L_{0}$ gelagert“.

Dabei ergeben sich die $\alpha_{i}$ aus einer Anpassung der analytischen Formel an die numerisch berechneten Eigenfrequenzwerte (,curve fitting“) zu etwa $1 / 4$, und die $\psi_{i}$ sind die Eigenwerte der Biegeknickdifferentialgleichung [Pfe92, Sza56]

$$
E I \frac{\partial^{4} w}{\partial x^{4}}-F \frac{\partial^{2} w}{\partial x^{2}}=0
$$

des Rohres. Durch Aufstellung des charakteristischen Polynoms der linearen homogenen Differentialgleichung erhält man die allgemeine Lösung [Bro87]

$$
w_{i}(x)=c_{1} \cos \psi_{i} x+c_{2} \sin \psi_{i} x+c_{3} \psi_{i} x+c_{4}
$$

Zur Ermittlung der speziellen Lösung werden die Lösungsfunktionen wieder bereichsweise definiert. Die Eigenwerte sind für jeden Abschnitt wiederum 
identisch, da es sich jeweils um ein und denselben Körper handelt. Die Randbedingungen sind identisch mit denen bei der Lösung der Schwingungsdifferentialgleichung. Die sich ergebenden kritischen Werte für das Axiallastverhältnis sowie die genauen Exponenten $\alpha_{i}$ sind ebenfalls in Tabelle 3-3 bis Tabelle 3-5 dargestellt. Die Berechnungen der analytischen Lösungen wurden unter Zuhilfenahme des Mathematik-Programms Mathematica 3.0.1 [Wo197, Fis00b] durchgeführt.

\subsection{Feststellbare Schwingungsphänomene}

\subsubsection{Einzelwirbelanregung}

Die Frequenzen anregender Einzelwirbel bzw. Familien von Ablösewirbeln können in den Fourier-Transformationen der Beschleunigungszeitreihen erkannt werden. Die Spektren stellen sich als mit dem Volumenstrom nach rechts hin zu höheren Frequenzen wandernde Peaks dar, die wegen der gleichmäßigen Steigerung der Volumenströme um jeweils $10 \mathrm{~m}^{3} / \mathrm{h}$ und des linearen Zusammenhangs zwischen Ablösefrequenz und Anströmgeschwindigkeit einerseits einen konstanten Frequenzabstand zueinander haben, aber andererseits eine nahezu gleichhohe Amplitude. Die Lage von Peaks im Frequenzdiagramm infolge der Anregung einer Rohreigenfrequenz bleibt hingegen bei Variation der Anströmgeschwindigkeit unverändert, während die Amplitude mit ansteigender Anströmgeschwindigkeit zunimmt. Eine Überlagerung der beiden Effekte ist möglich und wurde bei einigen Rohrbündeln beobachtet.

Bei den doppelt gelagerten Randrohren liegt die Größe der Fourierkoeffizienten, die Einzelwirbelfamilien zuzuordnen sind, in derselben Größenordnung wie diejenigen Peaks, die Rohreigenfrequenzen zugeordnet werden können. Die Strömungsgeschwindigkeiten sind in diesem Bereich so gering, daß das Anschlagen der Rohre in den Lagern nicht wesentlich bedeutsamer wird als die strömungsmechanischen Anregungsmechanismen. Ein resonantes Verhalten kann experimentell nicht festgestellt werden.

Anders verhält es sich bei den einfach gelagerten Rohren und doppelt gelagerten Zentralrohren. Diese erfahren wesentlich höhere Spaltgeschwindigkeiten. Außerdem sind die Eigenfrequenzen der einfach gelagerten Rohre deutlich niedriger. Erwartungsgemäß ergibt sich aus den Messungen der Fouriertransformierten dieser Rohre, daß Einzelwirbelpeaks nicht aufgelöst werden können, sondern die Fouriertransformation wird von den Peaks der Rohreigenfrequen- 
zen bestimmt. Hier ist das Anschlagen der Rohre in den Leitblechen der dominante Erregermechanismus.

Experimentell kann die Lock-In-Grenze durch langsame Variation der Anströmgeschwindigkeit ermittelt werden. Im Verlauf der RMS-Beschleunigungen in Abhängigkeit der Anströmgeschwindigkeit kann der Lock-In als signifikanter Peak ausgemacht werden. Die Erhöhung ist auf einen sehr schmalen Bereich der Anströmgeschwindigkeit begrenzt und kann bei weiterer Erhöhung der Anströmgeschwindigkeit wieder stark verringert werden, bevor die fluidelastische Instabilität einsetzt.

\subsubsection{Fluidelastische Instabilität}

Die fluidelastische Instabilität wäre grundsätzlich ebenso wie der Lock-InBereich einer Messung zugänglich. Allerdings waren die in der vorliegenden Arbeit verwendeten Meßsensoren auf den Bereich der stabilen Schwingungen unterhalb der Flattergrenze ausgelegt und wären bei fluidelastischer Instabilität zerstört worden. Deshalb mußte die Stabilitätsgrenze, die bei allen Bündeln rechnerisch (Kapitel 4) am oberen Ende des Variationsbereichs der Anströmgeschwindigkeit liegt, in einem gesonderten Versuch ohne Sensorbestückung rein akustisch bestimmt werden. Hierzu mußte die Anströmgeschwindigkeit so lange erhöht werden, bis das plötzliche instabile Anschlagen der Rohre in den Leitblechen als signifikantes Kennzeichen der fluidelastischen Instabilität auftrat.

\subsubsection{Dominante Eigenfrequenz}

Experimentell konnte nachgewiesen werden, daß durchaus nicht immer die erste Eigenfrequenz bereits die dominierende ist. Vielmehr wird insbesondere bei den einfach gelagerten Rohren in Erweiterung der bisher verwendeten Theorie die dritte Eigenfrequenz signifikant höher angeregt als alle anderen Eigenfrequenzen. Dies liegt in der fortwährenden Anregung durch die Schläge in den Bohrungen der Stützbleche bei einem Drittel der Rohrlänge begründet.

\subsubsection{Turbulenzanregung}

Alle weiteren feststellbaren Rohrbewegungen können auf Turbulenzanregung zurückgeführt werden. Die Beschleunigungsamplituden der Rohre nehmen mit zunehmender Strömungsgeschwindigkeit linear zu. Dies entspricht dem normalen Betriebsfall, bei dem stabile Schwingungen infolge Turbulenzerregung zu einem Langzeitschaden führen können. 


\subsection{Validierung der Messungen anhand theoretischer Überlegungen}

Nachdem es sich bei der vorliegenden Arbeit um ein außerordentlich komplexes Schwingungsproblem handelt, wurden Versuche unternommen, die Meßergebnisse unabhängig von den Simulationsergebnissen zu verifizieren.

\subsubsection{Vergleich bei großen Teilungsverhältnissen}

Als erster Ansatz wurde hierzu ein analytischer Zusammenhang zwischen den Spalt-Strouhalzahlen der verschiedenen Eigenfrequenzen der Rohre und den Spalt-Reynoldszahlen ermittelt [Kas97a]. Die auf die Spaltgeschwindigkeit bezogene Strouhalzahl einer Eigenfrequenz lautet

$$
S r_{s p, i}=\frac{f_{i} D}{u_{s p}}
$$

Die zugehörige Reynoldszahl ist

$$
R e_{s p}=\frac{u_{s p} D \rho}{\eta}
$$

Durch Elimination der Spaltgeschwindigkeit läßt sich mit

$$
S r_{s p, i}=\frac{f_{i} D^{2} \rho}{\eta} \frac{1}{R e_{s p}}
$$

ein dimensionsloser Zusammenhang zwischen der Spaltstrouhalzahl und der Spaltreynoldszahl herstellen. Es ergibt sich eine Hyperbel mit einem für jede Ordnung von Eigenfrequenzen und Außenrohrdurchmesser unterschiedlichen Vorfaktor.

Entscheidend für die Berechnung der Eigenfrequenz ist der hydrodynamische Zusatzfaktor $k$. Dieser muß so angepaßt werden, daß die dominant angeregte Eigenfrequenz korrekt berechnet wird, hier die dritte Eigenfrequenz. In Abbildung 3-10 und Abbildung 3-11 sind für große Teilungsverhältnisse $\tau$ die entsprechenden Hyperbeln für die entsprechenden Rohrbündel und Lagerungsfälle für Bohrbilder BB1 und BB2 dargestellt. Die mit den gemessenen Eigenfrequenzen gebildeten Werte gruppieren sich um die theoretischen Kurven für den maximal und minimal auftretenden hydrodynamischen Zusatzkoeffizienten $k$ herum [Sto00]. 


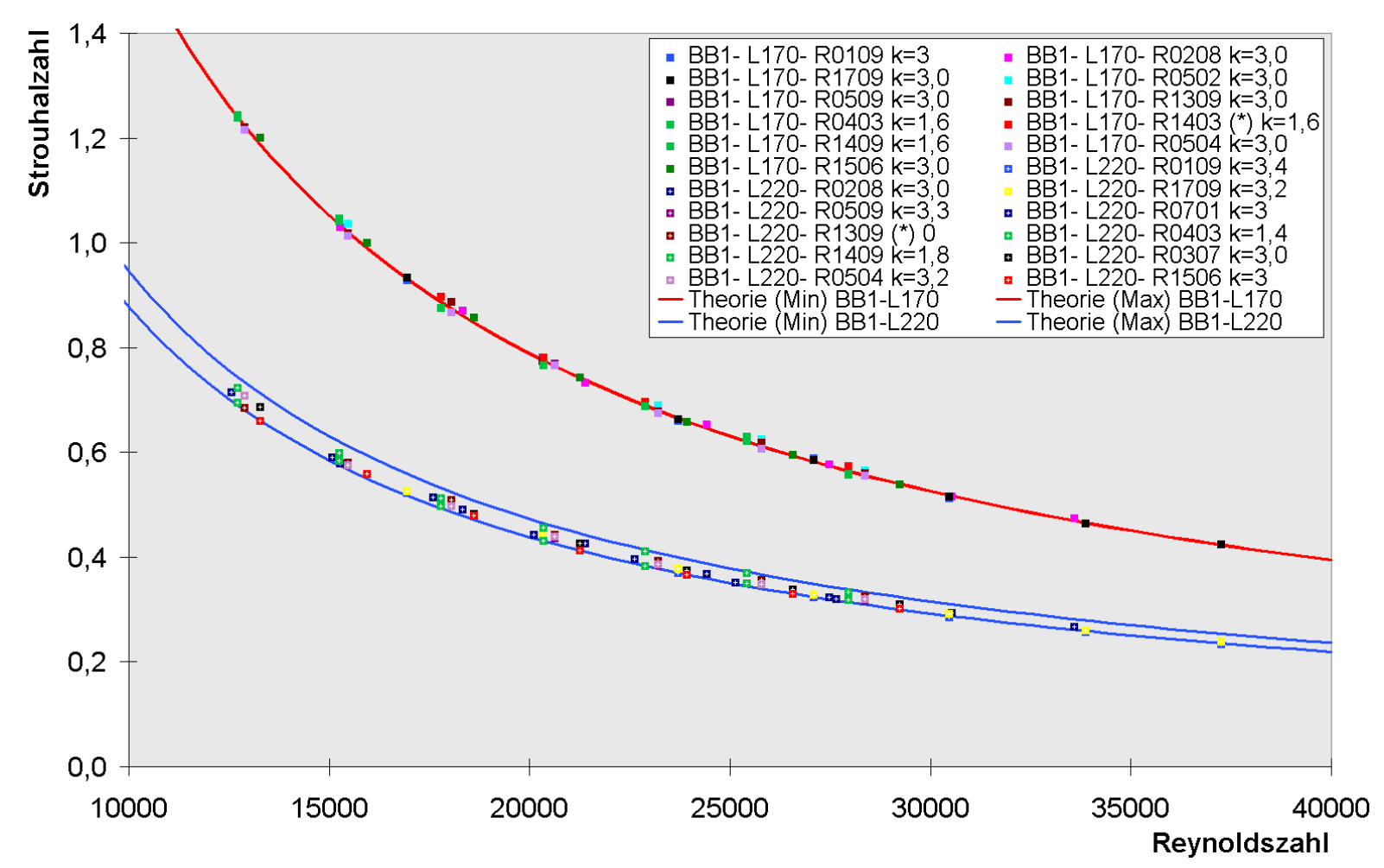

Abbildung 3-10: $S r-R e$-Diagramm für die dritte angeregte Eigenfrequenz der Bohrbilder BB1 bei einfacher Lagerung.

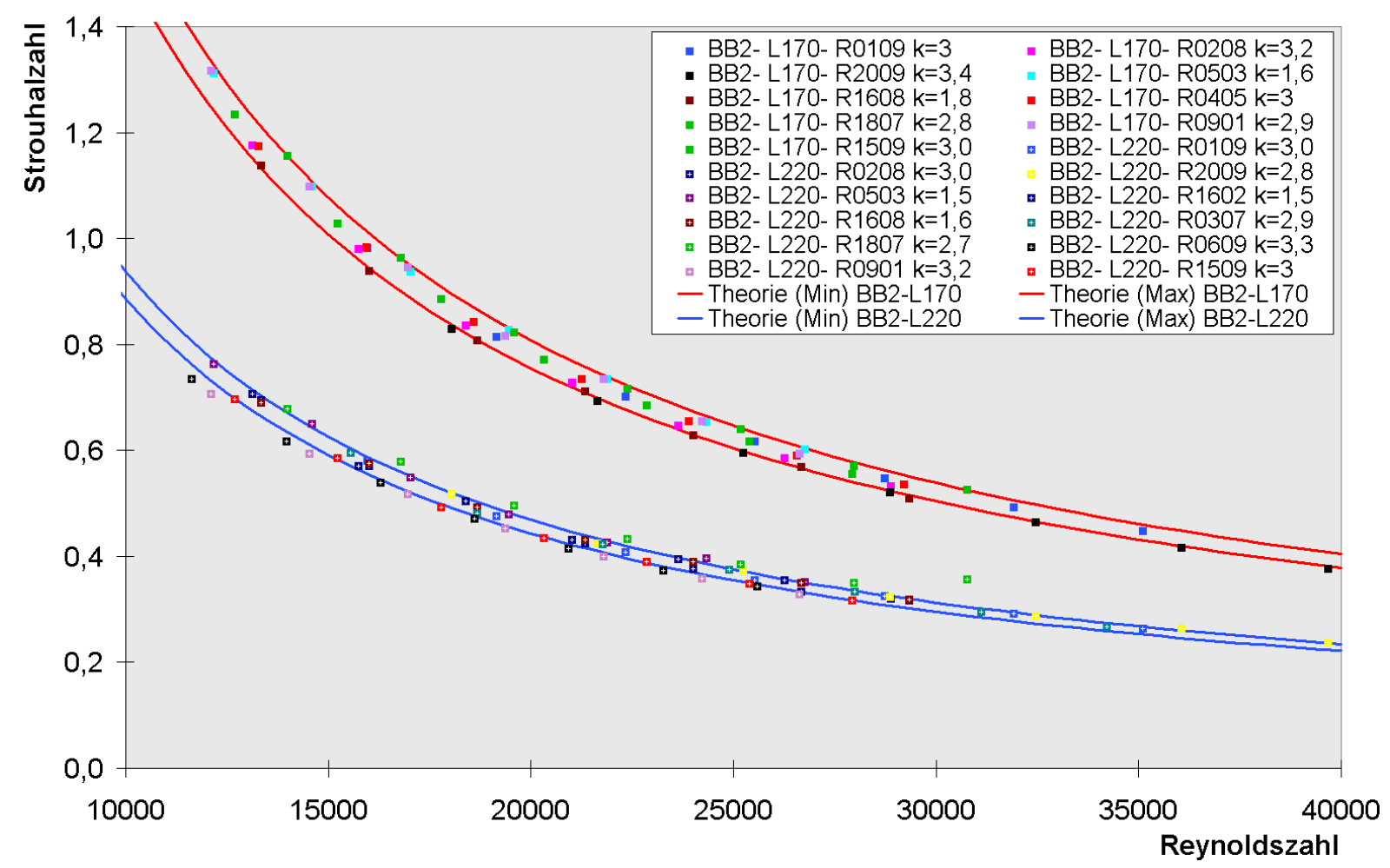

Abbildung 3-11: Sr-Re-Diagramm für die dritte angeregte Eigenfrequenz der Bohrbilder BB2 bei einfacher Lagerung. 


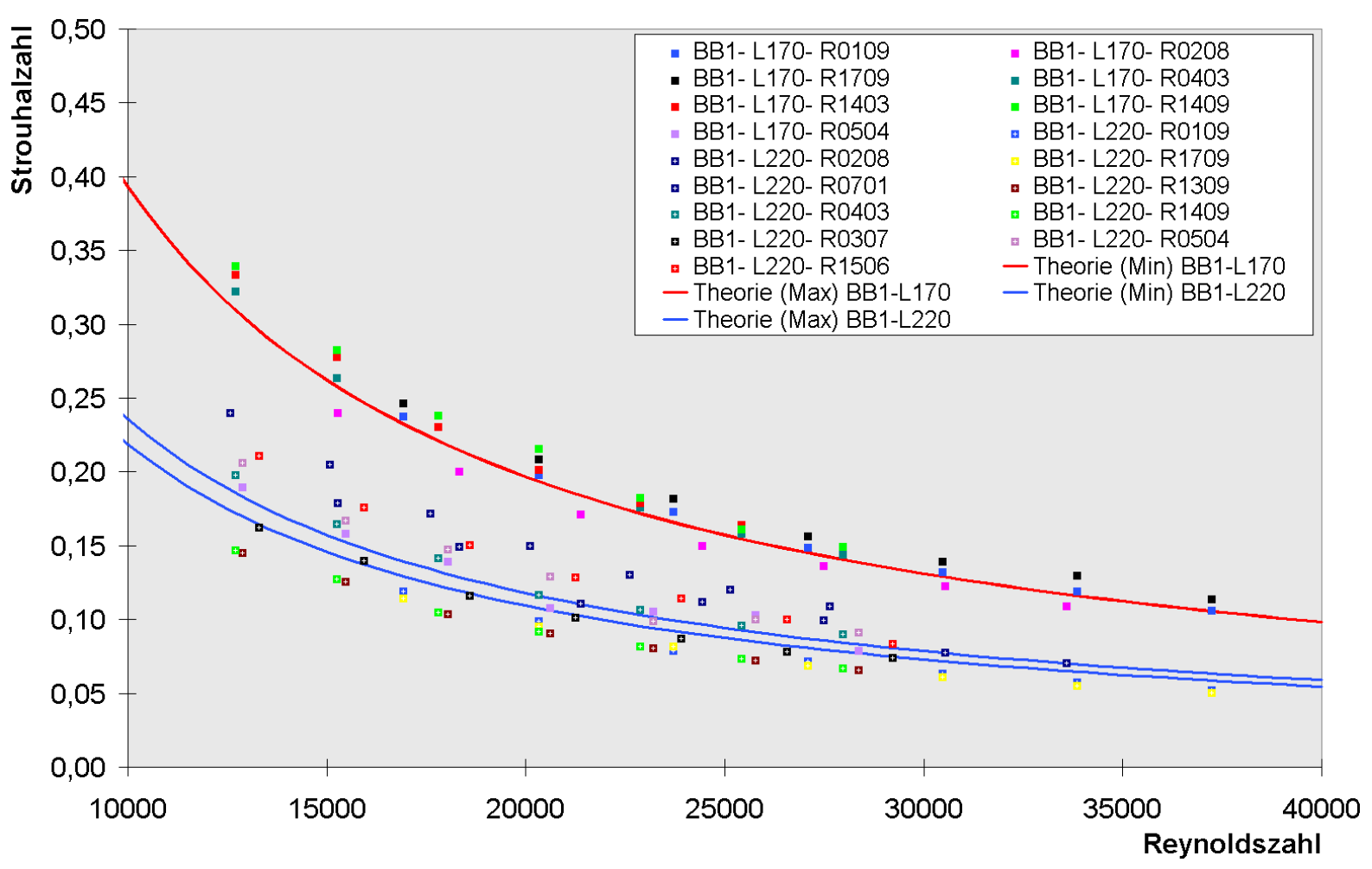

Abbildung 3-12: $S r$-Re-Diagramm für die erste angeregte Eigenfrequenz der Bohrbilder BB1 bei einfacher Lagerung.

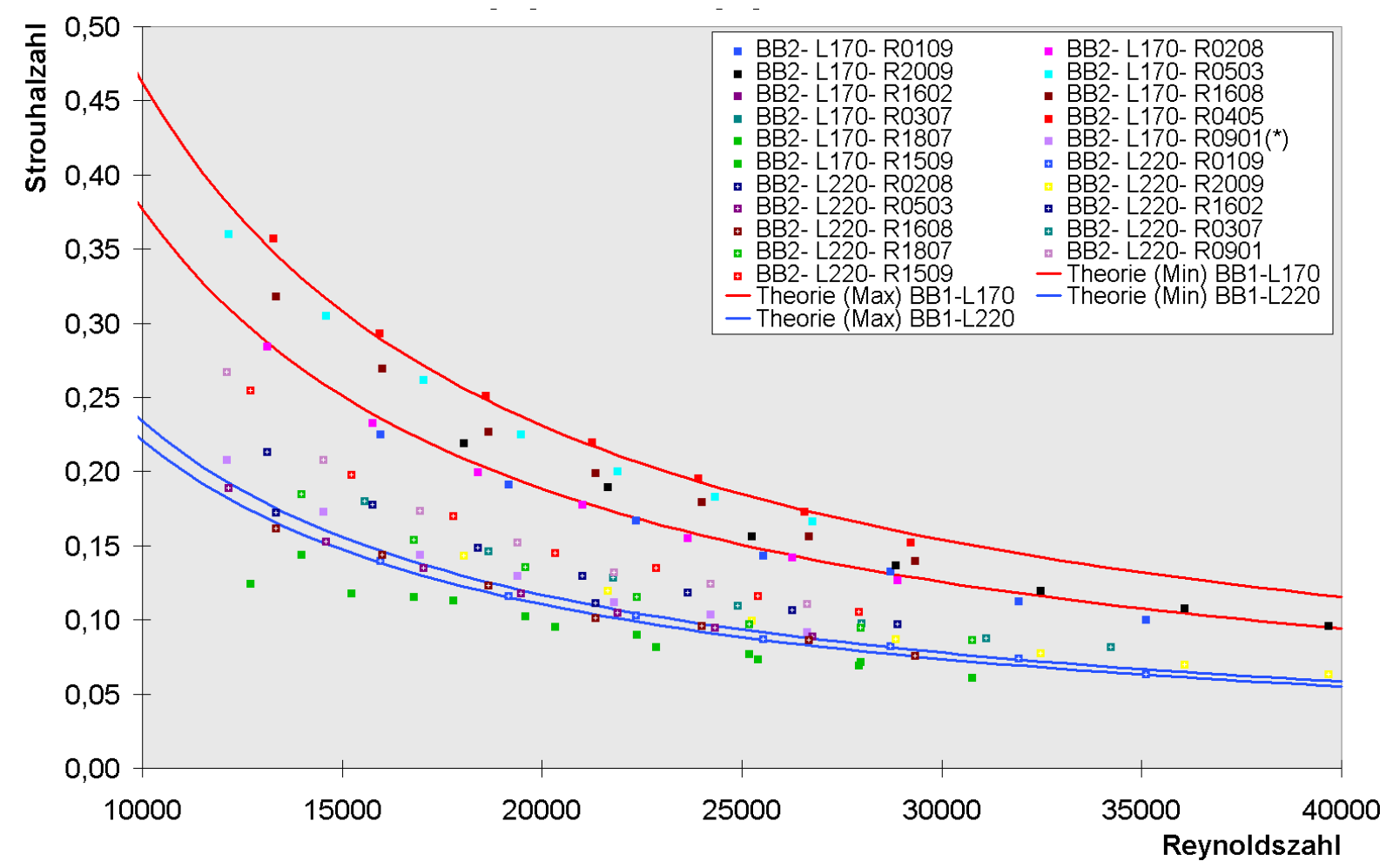

Abbildung 3-13: $S r$-Re-Diagramm für die erste angeregte Eigenfrequenz der Bohrbilder BB2 bei einfacher Lagerung. 
Die gemessenen Eigenfrequenzen wurden aus den charakteristischen Fouriertransformationen abgelesen. Die Zuordnung der einzelnen Peaks zu den entsprechenden Eigenfrequenzen konnte durch die Verwendung der analytischen Lösungen für die Eigenfrequenzen ermittelt werden. Eine Variation der Spaltgeschwindigkeit bewirkt lediglich eine Verschiebung aller Meßpunkte entlang der theoretischen Hyperbeln [Sto00], daher wurde o.B.d.A. eine homogene Kammerndurchströmung angenommen.

Die Übereinstimmungen zwischen den Meßergebnissen und den theoretischen Kurven zeigen für große Teilungsverhältnisse, daß hinsichtlich der Frequenzbestimmung richtig gemessen wurde. Die dimensionslose Formulierung der $\mathrm{Zu}$ sammenhänge liefert dabei eine sehr übersichtliche Präsentationsform. Das Konzept orientiert sich am Phänomen der Einzelwirbelablösung. Für kleine Teilungsverhältnisse ist daher eine $S r$-Re-Darstellung nicht möglich [Kas97a].

\subsubsection{Kritik am Konzept der hydrodynamischen Zusatzmassen}

Hinsichtlich des theoretischen Konzepts des hydrodynamischen Zusatzfaktors $k$ offenbaren die Meßergebnisse für große Teilungsverhältnisse zwei grundsätzliche Schwächen. Zum einen ist der Koeffizient $k$ offensichtlich abhängig von der zu berechnenden Eigenfrequenz. Dies kann man daran erkennen, daß die Ergebnisse im Vergleich zwischen Theorie und Messungen für die auf den Fall der dritten angeregten Eigenfrequenz angepaßten Werte für $k$ hervorragend übereinstimmen. Bildet man mit denselben $k$ dieselben Diagramme z.B. für die erste Eigenfrequenz wie in Abbildung 3-12 und Abbildung 3-13, so zeigt sich eine wesentlich größere Streuung [Sto00].

Daß $k$ von der Schwingungsfrequenz abhängt, ist auch schon im Zusammenhang mit Schwingungsmessungen an Rührwerken bekannt geworden [Bur71]. Dort wurde für höhere Eigenfrequenzen ein Abfall des hydrodynamischen $\mathrm{Zu}$ satzkoeffizienten festgestellt. Erklärbar ist dieses Phänomen dadurch, daß höhere Schwingungsfrequenzen mit höheren Rohrgeschwindigkeiten einhergehen, das Fluid aber aufgrund seiner Trägheit immer schlechter folgen kann.

Die zweite Schwäche des Konzeptes der hydrodynamischen Koeffizienten zeigt sich, wenn man die sich aus den Messungen ergebenden $k$-Faktoren für die vier vermessenen Rohrbündel mit großen Teilungsverhältnissen in Abbildung 3-14 und Abbildung 3-15 betrachtet. Dabei erkennt man, daß der Faktor $k$ für praktisch alle vermessenen Rohre einen Wert um 3,0 annimmt. Rohre, die unmittelbar vor einem Leitblech liegen, erreichen nur noch Werte um 1,5 [Sto00]. Der 
Faktor $k$ beschreibt mit Hilfe eines erhöhten Trägheitsanteils die Interaktion zwischen den Rohren eines Bündels, die bei großen Teilungsverhältnissen theoretisch quer zur Anströmung gegeneinander schwingen. Ein schwingendes Rohr muß nun nicht nur die umgebende Fluidmasse verdrängen, sondern zusätzlich noch gegen die vom entgegenschwingenden Partnerrohr heranbeförderten Fluidmassen arbeiten. Daher ist $k$ immer größer als 1,0. Auf Höhe des Leitblechs existiert jedoch keine Bewegung des Gegenrohrs. Damit muß sich der zusätzliche Trägheitsanteil auch etwa halbieren.

Hinsichtlich der in [TEM82, VDI97] propagierten Berechnungsmethode zur Bestimmung der Rohreigenfrequenzen ergeben sich damit drei Kritikpunkte:

1. Die in Diagrammform für verschiedene Teilungsverhältnisse dargestellten hydrodynamischen Zusatzkoeffizienten gelten nur für die erste Eigenfrequenz und differieren auch zwischen [TEM82] und [VDI97].

2. Im vorliegenden Problemfall war jedoch die dritte Eigenfrequenz dominant. Hierfür gelten aber offenbar aufgrund der vorliegenden Meßergebnisse die in [TEM82, VDI97] angegebenen Faktoren $k$ nicht bzw. nicht mehr genau.

3. In der Nähe von Einbauten reduzieren sich die Faktoren $k$, im vorliegenden Fall halbieren sie sich etwa an Leitblechkanten. Welche zusätzlichen Belastungen sich durch dieses Absinken etwa durch Schwebungserscheinungen ergeben könnten, bleibt durch die Vorschriftenrechnungen unberücksichtigt.
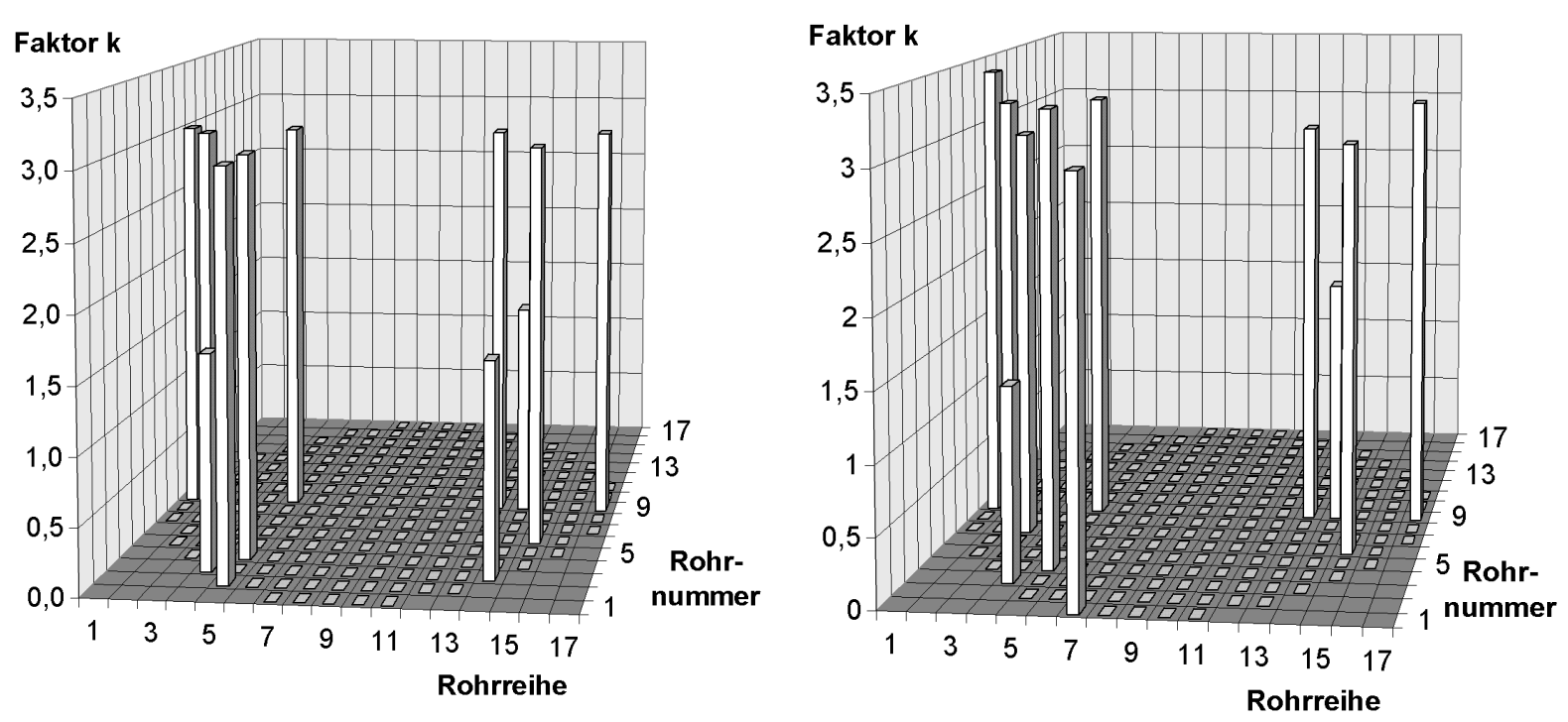

Abbildung 3-14: Gemessener hydrodynamischer Zusatzfaktor $k$ für die dritte Eigenfrequenz. Links BB1-L170, rechts BB1-L220. 
Die numerische Simulation bedarf nicht mehr der Annahme eines hydrodynamischen Zusatzkoeffizienten. Da das äußere Strömungsfeld simuliert wird, sind eigenfrequenzdämpfende Kräfte auf das Rohr in der Simulation implizit enthalten. Die Auswahl einer dominanten Eigenfrequenz wird unnötig. Ungenauigkeiten in der Bestimmung der Dämpfung werden methodisch ausgeschlossen.
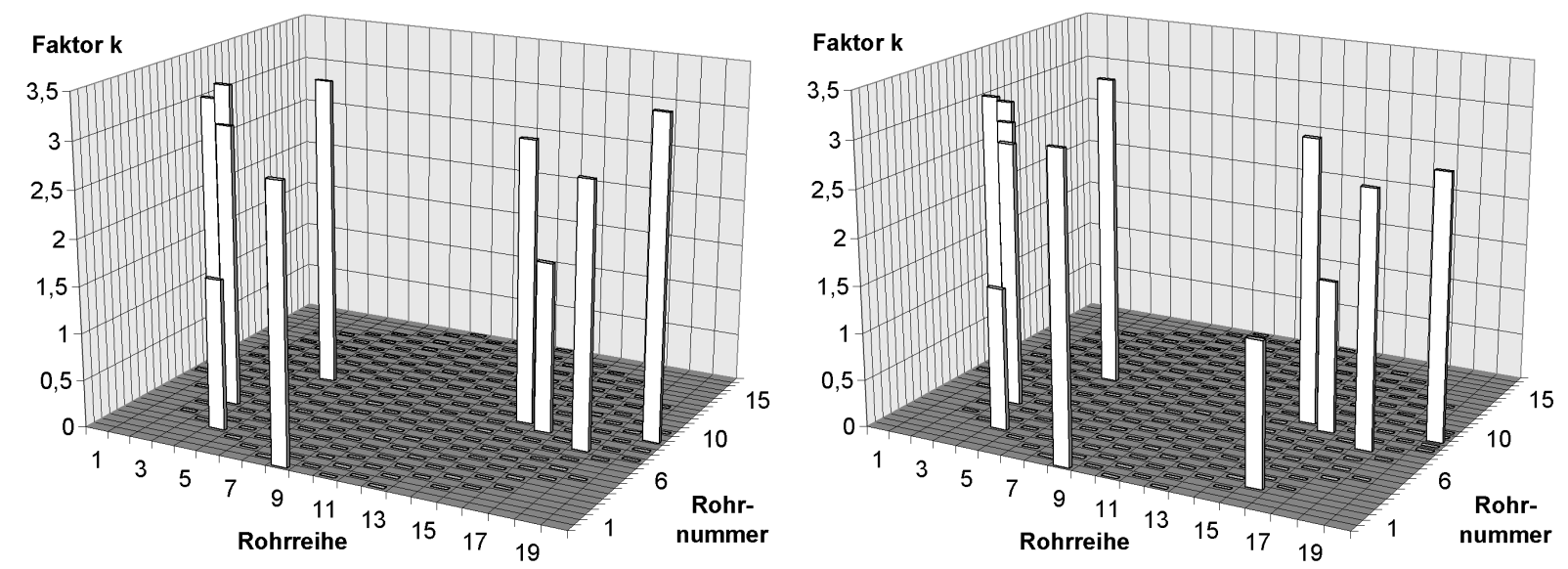

Abbildung 3-15: Gemessener hydrodynamischer Zusatzfaktor $k$ für die dritte Eigenfrequenz. Links BB2-L170, rechts BB2-L220.

\subsubsection{Erweiterung des Theorievergleichs auf kleine Teilungsverhältnisse}

Probleme bei der Bewertung der Messungen mit Hilfe von Sr-Re-Diagrammen ergaben sich bei kleinen Teilungsverhältnissen, also insbesondere für die Bohrbilder BB3 und BB4. Deshalb wurde in [Sto00] eine weitere analytische Methode zur Beurteilung der Messungen entwickelt. Grundlage der Beurteilung sind die RMS-Verteilungen für jedes Rohr entlang der Rohrlängsachse sowie die charakteristischen Fouriertransformierten. Dabei werden zunächst in Fortführung der analytischen Berechnung der Eigenfrequenzen die Eigenformen analytisch bestimmt, die sich für die drei Lagerungsfälle für die sechs ersten Eigenfrequenzen ergeben. Die Herleitung hierzu ist detailliert in [Sto00] enthalten. Welche der Eigenformen wie stark angeregt werden, ergibt sich aus der Höhe der zugehörigen Peaks der charakteristischen Fouriertransformation für jedes Rohr. Stolz [Sto00] gewinnt eine theoretische Gesamteigenform der Rohre durch Wichtung der normierten analytischen Eigenformen mit ihren zugehörigen gemessenen Fouriermaxima. Dies entspricht in gewisser Weise einer unvollständigen Rücktransformation vom Frequenzraum in den Ortsraum. 
In Abbildung 3-16 ist für einen zufällig ausgewählten Fall bei einem Volumenstrom von $110 \mathrm{~m}^{3} / \mathrm{h}$ eine Gegenüberstellung von gemessenen normierten RMSBeschleunigungsverläufen und theoretisch berechneten Eigenformen unter Berücksichtigung der gemessenen Fourierkoeffizienten für die einzelnen Eigenfrequenzen dargestellt. Bei den gemessenen Verläufen müssen die Beschleunigungen in den Leitblechen zum Vergleich mit der Theorie auf Null gesetzt werden, da der Fall des Anschlagens in den Leitblechen nicht in den analytischen Überlegungen enthalten ist. Die normierten RMS-Meßkurven stehen in guter Übereinstimmung mit den theoretisch zu erwartenden Kurven für die normierten Eigenformen. Die Eigenformen der Rohre können deswegen mit den RMSBeschleunigungen in Beziehung gesetzt werden, weil bei der zweimaligen zeitlichen Ableitung des Produktansatzes (3-12) der Lösung der Bewegungsgleichung der Rohre lediglich der zeitabhängige Faktor betroffen ist, an der Form des ortsabhängigen Anteils der Lösung ändert sich hingegen nichts.

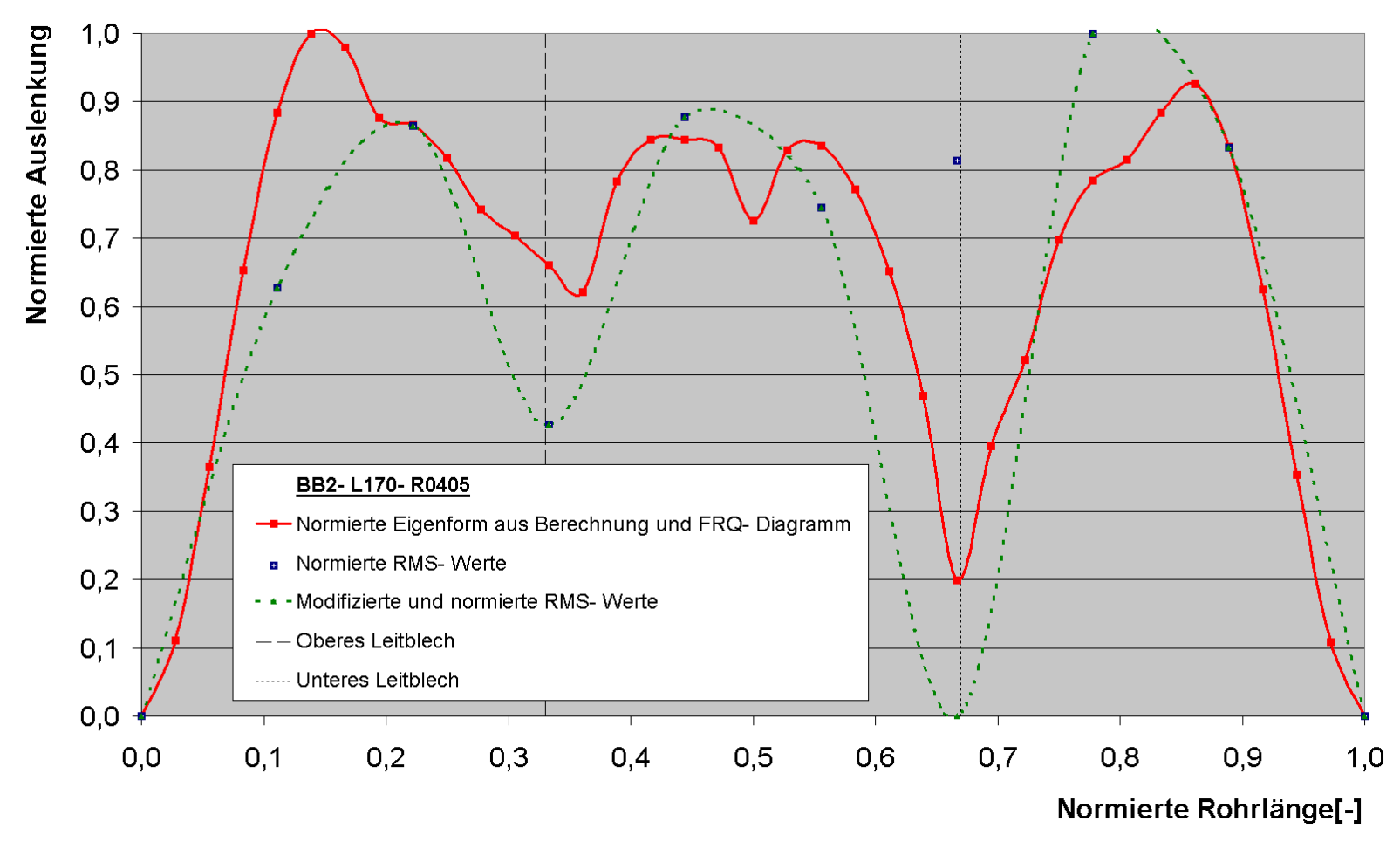

Abbildung 3-16: Vergleich gemessener und theoretisch ermittelter RMS-Beschleunigungsverteilungen entlang der Rohrlängsachse für BB2-L170-R0405.

Die Methode konnte erfolgreich für Rohrbündel der Bohrbilder BB1, BB2 und BB4 angewendet werden. Auch wenn es in einigen Fällen, insbesondere bei den Rohrbündeln mit Bohrbild BB3, aufgrund der komplizierten sich ergebenden 
Fourierdiagramme nicht möglich war, die gemessenen Werte eindeutig mit theoretischen Überlegungen zu kombinieren, so konnten doch die Messungen von 40 der 85 fehlerfrei vermessenen Rohre unabhängig von den Simulationsergebnissen auf rein theoretischer Basis hinsichtlich der Richtigkeit der Frequenzbestimmung überprüft werden. Das dargestellte Auswerteverfahren lieferte damit für eine ausreichend große Stichprobe der vermessenen Rohre die erwünschte deutliche Bestätigung, daß bei den Messungen hinsichtlich der Frequenz kein systematischer Fehler vorlag.

Die absolute Höhe des Beschleunigungsmeßwertes ist so jedoch nicht nachprüfbar. Die analytischen Lösungen der Rohrbewegungsgleichungen sind nur bis auf einen Vorfaktor bestimmt. Dies liegt darin begründet, daß nur die Lösung einer homogenen partiellen Differentialgleichung (3-13a) gesucht wurde, die zur Lösung eines homogenen Gleichungssystems führt. Homogene Gleichungssysteme haben jedoch immer nur nichttriviale Lösungen, die nur bis auf einen Vorfaktor bestimmbar sind. Daher werden in der dargestellten Methode auch nur normierte Darstellungen miteinander verglichen. Für die Bestimmung des Vorfaktors wäre eine Bestimmung der Lösung der inhomogenen Biegeschwingungsdifferentialgleichung (2-61) notwendig gewesen, bei der der Störterm von den orts- und zeitabhängigen äußeren Belastungen der Rohre abhängig ist. Diese äußeren Belastungen sind aber ja theoretisch eben nicht bekannt. Gerade sie sollen ja mit dem numerischen Simulationsverfahren aus Kapitel 2 ermittelt werden. 


\section{Berechnungsbeispiele}

\subsection{Relevante Schadensmechanismen}

Nach bisherigem Stand der Technik (z.B. [VDI97]) wird eine Absicherung eines Wärmetauschers gegen Schwingungsschädigungen durch einen der folgenden vier Schadensmechanismen durchgeführt:

- Einzelwirbelanregung

- Fluidelastische Instabilität

- Turbulenzanregung

- Akustische Resonanz

Grundlage der Beurteilung der Schwingungsgefährdung eines Rohrbündels hinsichtlich Einzelwirbelanregung und fluidelastischer Instabilität sind die Spaltgeschwindigkeitsverteilungen der Strömung entlang der Rohre im Mantelraum und die Eigenfrequenzen der Rohre. Es wird dann verglichen, ob die kritischen Strömungsgeschwindigkeiten erreicht werden oder nicht. Eine Aussage über die Höhe der Schwingungsamplituden ist nach bisherigem Stand der Technik nicht möglich.

Turbulente Anregung führt zwar zu einem erhöhten Lagerverschleiß, soll aber nicht überprüft werden, da dies nicht Gegenstand der vorliegenden Arbeit ist. Hierfür wäre die Kenntnis des Turbulenzspektrums erforderlich [VDI97].

Akustische Resonanz muß im vorliegenden Fall nicht überprüft werden, da diese nur bei Gasströmungen auftritt [VDI97]. Es verbleiben als zu überprüfende Schadensmechanismen die Einzelwirbelanregung und die fluidelastische Instabilität.

\subsection{Bisherige halbempirische Absicherung}

\subsubsection{Bestimmung der Rohreigenfrequenzen}

Für die Beurteilung der Schwingungsgefährdung müssen in allen Verfahren zunächst die Eigenfrequenzen der Rohre im Bündel bestimmt werden. Sie werden entsprechend Kapitel 3 analytisch berechnet [Fis00b]. Die hydrodynamischen Zusatzkoeffizienten werden für das vorliegende Teilungsverhältnis von $\tau=1,6$ (BB1 und BB2) aus dem TEMA-Standard [TEM82] zu $k=1,8$ und für $\tau=1,2$ (BB3 und BB4) zu $k=3,2$ bestimmt. In Tabelle 4-1 bis Tabelle 4-4 sind die ersten sechs Eigenfrequenzen für die Lagerfälle „freies Rohr“, „einfach asymme- 
trisch gelagertes Rohr“ und „Zweifach symmetrisch gelagertes Rohr“ für die Rohrlängen 1,6 m und 2,1 m sowie die Rohraußendurchmesser $12 \mathrm{~mm}$ und $16 \mathrm{~mm}$ zusammengestellt. Die hierfür notwendigen Eigenwerte der Biegeschwingungsdifferentialgleichung werden Tabelle 3-3 bis Tabelle 3-5 entnommen [Fis00b]. Der E-Modul der verwendeten Aluminiumlegierung wurde durch eigene Messungen zu $69000 \mathrm{~N} / \mathrm{mm}^{2}$ bestimmt, die Dichte des Rohrwerkstoffs beträgt nach eigenen Messungen $2587 \mathrm{~kg} / \mathrm{m}^{3}$. Die mitbewegte Masse ergibt sich damit über die Berücksichtigung der Wasserdichte von $1000 \mathrm{~kg} / \mathrm{m}^{3}$.

\begin{tabular}{|c|c|c|c|c|c|c|}
\hline Lager & $f_{1}$ & $f_{2}$ & $f_{3}$ & $f_{4}$ & $f_{5}$ & $f_{6}$ \\
\hline 0 & 13,8 & 37,9 & 74,4 & 122,9 & 183,6 & 256,5 \\
\hline 1 & 26,5 & 72,7 & 106,3 & 158,7 & 253,7 & 312,4 \\
\hline 2 & 70,0 & 102,3 & 123,9 & 249,1 & 305,5 & 341,1 \\
\hline
\end{tabular}

Tabelle 4-1: Rohreigenfrequenzen $f_{1}$ bis $f_{6}$ in $\mathrm{Hz}$ für Bohrbilder BB1-L170 und BB2-L170.

\begin{tabular}{|c|c|c|c|c|c|c|}
\hline Lager & $f_{1}$ & $f_{2}$ & $f_{3}$ & $f_{4}$ & $f_{5}$ & $f_{6}$ \\
\hline 0 & 13,9 & 38,3 & 75,1 & 124,2 & 185,5 & 259,1 \\
\hline 1 & 26,8 & 73,5 & 107,4 & 160,3 & 256,4 & 315,6 \\
\hline 2 & 70,7 & 103,3 & 125,1 & 251,7 & 308,7 & 344,7 \\
\hline
\end{tabular}

Tabelle 4-2: Rohreigenfrequenzen $f_{1}$ bis $f_{6}$ in $\mathrm{Hz}$ für Bohrbilder BB3-L170 und BB4-L170.

\begin{tabular}{|c|c|c|c|c|c|c|}
\hline Lager & $f_{1}$ & $f_{2}$ & $f_{3}$ & $f_{4}$ & $f_{5}$ & $f_{6}$ \\
\hline 0 & 7,9 & 21,8 & 42,7 & 70,6 & 105,4 & 147,2 \\
\hline 1 & 15,2 & 41,8 & 61,0 & 91,1 & 145,7 & 179,3 \\
\hline 2 & 40,6 & 59,4 & 71,9 & 144,6 & 177,3 & 198,0 \\
\hline
\end{tabular}

Tabelle 4-3: Rohreigenfrequenzen $f_{1}$ bis $f_{6}$ in $\mathrm{Hz}$ für Bohrbilder BB1-L220 und BB2-L220. 


\begin{tabular}{|c|c|c|c|c|c|c|}
\hline Lager & $f_{1}$ & $f_{2}$ & $f_{3}$ & $f_{4}$ & $f_{5}$ & $f_{6}$ \\
\hline 0 & 8,1 & 22,2 & 43,6 & 72,1 & 107,7 & 150,4 \\
\hline 1 & 15,5 & 42,7 & 62,3 & 93,1 & 148,8 & 183,2 \\
\hline 2 & 41,1 & 60,0 & 72,6 & 146,1 & 179,2 & 200,1 \\
\hline
\end{tabular}

Tabelle 4-4: Rohreigenfrequenzen $f_{1}$ bis $f_{6}$ in $\mathrm{Hz}$

für Bohrbilder BB3-L220 und BB4-L220.

\subsubsection{Bestimmung des Strömungsfeldes im Mantelraum}

Sowohl für die Beurteilung der Schwingungsgefährdung infolge von Einzelwirbelanregung als auch für die Beurteilung der Gefährdung durch fluidelastische Instabilität ist die Kenntnis der Strömungsverteilung im Mantelraum notwendig. Aus ihr können die herrschenden Spaltgeschwindigkeiten entlang der Rohrlängsachsen ermittelt werden, um einerseits mit Hilfe der in Diagrammen [VDI97] verfügbaren Spalt-Strouhalzahlen auf die herrschenden Ablösefrequenzen von Einzelwirbeln zu schließen und andererseits den Belastungsfaktor $S$ als Eingangsgröße für die gängigen erweiterten halbempirischen Kriterien (1-1) und (1-2) ermitteln und die herrschende mit der kritischen Spaltgeschwindigkeit vergleichen zu können.

Früher wurden die Geschwindigkeitsverteilungen nur grob sektionsweise unter Zuhilfenahme der Kontinuitätsgleichung abgeschätzt. Inzwischen ist es aber Stand der Technik (vgl. Kapitel 1.4, insbesondere [Pri98, Bot00, Fis98c, Gel98, Kaa00, Kre00, Urb94]), das Strömungsfeld im Mantelraum des Wärmetauschers mit Hilfe kommerzieller Software-Pakete (z.B. [AEA97]) stationär zu simulieren und die sich ergebenden Spaltgeschwindigkeiten in die halbempirischen Betrachtungen einfließen zu lassen [VDI97, Kas94].

Hierbei können prinzipiell zwei verschiedene Modellierungswege beschritten werden. Zum einen kann das Rohrbündel unter Einbeziehung aller Geometrieelemente genau nachmodelliert werden [Fis98c, Gel98, Kaa00, Kre00, Urb94], dies erfordert jedoch eine lange Modellierungszeit und einen großen Speicher. Zum anderen kann das Bündel aber auch als Porosität modelliert werden [Pri98, Bot00], dadurch reduziert sich die Modellierungszeit drastisch. Die sich im porösen Bündel ergebenden Strömungsgeschwindigkeiten können dann direkt mit der gesuchten Spaltgeschwindigkeit identifiziert werden. Für die Zwecke der halbempirischen Stabilitätskriterien ist dieses Vorgehen ausreichend. 
Im vorliegenden Fall sollen alle acht experimentell untersuchten Bündel halbempirisch beurteilt werden, der Modellierungsaufwand wäre damit aber sehr hoch. Deshalb wird die zweite Modellierungsvariante gewählt, bei der die Bündel durch eine von ihrem Teilungsverhältnis bestimmte Porosität entsprechend dem Verhältnis des freien zum verdeckten Volumen im regulären Feld belegt werden. Die Leitbleche werden durch unendlich dünne strömungsundurchlässige Zelloberflächen dargestellt. Die Spaltströmungsgeschwindigkeiten ergeben sich aus der numerischen Simulation des Strömungsfeldes mit Hilfe des kommerziellen CFD-Codes CFX 4.1 [AEA97]. In Abbildung 4-1 ist als Beispiel das Geometrie-Modell von BB1-L170 und das Vektorfeld der Geschwindigkeit im Halbschnitt dargestellt. Die Strömungsgeschwindigkeiten wurden stationär am symmetrischen Halbschnittmodell ermittelt. Die Strömungsfelder in den anderen sieben Modellen wurden analog berechnet.
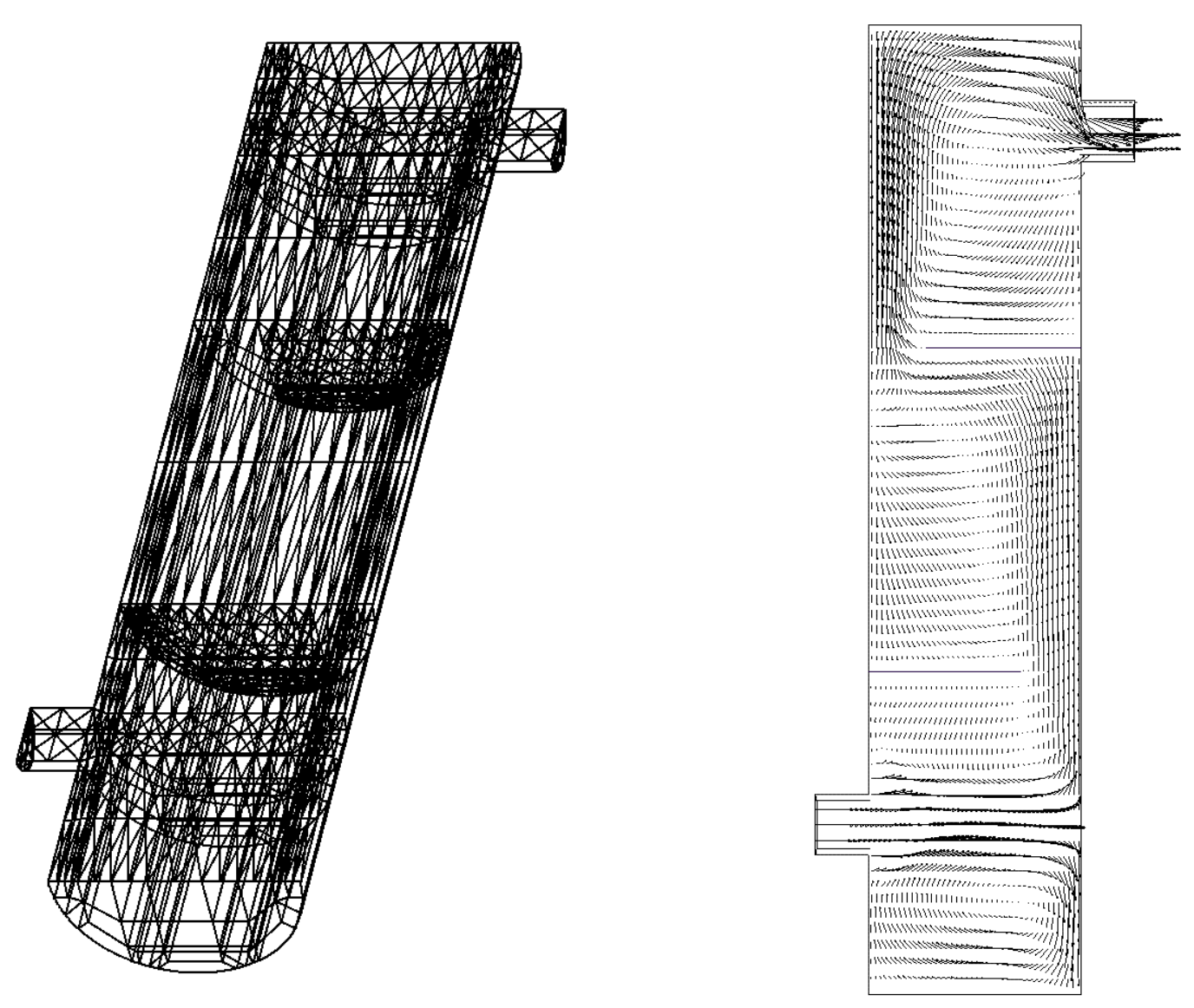

Abbildung 4-1: CFX-Modell von BB1-L170 und Vektorverteilung.

Bei der numerischen Simulation wurde in der vorliegenden Arbeit nur der maximale Lastfall von $110 \mathrm{~m}^{3} / \mathrm{h}$ Volumenstrom berücksichtigt. Bei den niedrigeren 
Volumenströmen (im Experiment 50 bis $110 \mathrm{~m}^{3} / \mathrm{h}$ in Schritten von $10 \mathrm{~m} / \mathrm{h}$ ) ergeben sich entsprechend niedrigere Spaltgeschwindigkeiten, die Strömungsgeschwindigkeitsverteilungen werden als zueinander ähnlich angenommen.

\subsubsection{Anregung durch Einzelwirbelablösung}

Um auf Einzelwirbelanregung überprüfen zu können, müssen die herrschenden Wirbelablösefrequenzen mit den Eigenfrequenzen der Rohre verglichen werden. Die Wirbelablösefrequenzen ergeben sich gemäß (3-26) aus den SpaltStrouhalzahlen, die für die verschiedenen Rohranordnungen in [VDI97] angegeben werden. Die Strouhalzahlen sind in Tabelle 4-5 aufgelistet. Bei 90-GradAnordnung sind für Teilungsverhältnisse kleiner als 1,4 keine Strouhalzahlen erster Ordnung und allgemein bei 90-Grad-Anordnung keine Strouhalzahlen zweiter Ordnung experimentell nachgewiesen worden. Einzelwirbelanregung ist damit für BB3 als Anregungsursache ausgeschlossen. Dies deckt sich mit dem experimentellen Befund, daß eine analytische Rekonstruktion der Eigenformen bei BB3 nicht möglich war, da diese auf der eindeutigen Identifizierbarkeit der Frequenzpeaks in den Meßdiagrammen beruhte. Dem Strömungsfeld wird nicht genug Platz geboten, um großskalige Wirbelstrukturen ausbilden zu können. Für BB4 mußte $S r_{1}$ im Diagramm extrapoliert werden, der Wert ist deshalb eingeklammert.

\begin{tabular}{|c|c|c|c|c|}
\hline Bohrbild & BB1 & BB2 & BB3 & BB4 \\
\hline$\tau$ & 1,6 & 1,6 & 1,2 & 1,2 \\
\hline Anordnung & $90 \mathrm{Grad}$ & $30 \mathrm{Grad}$ & $90 \mathrm{Grad}$ & $30 \mathrm{Grad}$ \\
\hline$S r_{1}$ & 0,12 & 0,35 & - & $(0,57)$ \\
\hline$S r_{2}$ & - & 0,51 & - & 0,80 \\
\hline
\end{tabular}

Tabelle 4-5: Spalt-Strouhalzahlen der untersuchten Rohrbündel [VDI97].

Hinsichtlich des Umfangs eines Vergleichs von Eigenfrequenzen und Wirbelablösefrequenzen können wiederum zwei verschiedene Wege beschritten werden. Zum einen kann in jedem Spalt die dominierende Strömungsgeschwindigkeit je Strömungssektion bestimmt werden. Hieraus ergibt sich die dominierende Wirbelablösefrequenz für jede Durchströmungsgeschwindigkeit. Aus der 
Betrachtung aller herrschenden Ablösefrequenzen im Betriebsbereich der Anströmgeschwindigkeiten ergibt sich für jede Sektion ein Spektrum von Einzelwirbeln, die für Schwingungserregung verantwortlich sein können.

Schwingungsresonanz (Lock-In) kann nur dann eintreten, wenn eine der dominierenden Rohreigenfrequenzen im Spektrum der dominierenden Wirbelablösefrequenzen enthalten ist oder höchsten $30 \%$ Frequenzabstand von den Grenzen des Spektrums hat. Als untere Grenze muß nach [VDI97] insbesondere die erste Eigenfrequenz berücksichtigt werden. Bei Anregung in Strömungsrichtung verdoppeln sich die berechneten Wirbelablösefrequenzen. Dadurch erweitert sich das Anregungsspektrum um einen Faktor Zwei.

\begin{tabular}{|c|c|c|c|c|c|c|c|c|c|}
\hline \multirow[b]{3}{*}{ Rohrbezeichnung } & \multicolumn{6}{|c|}{ Anregungsfrequenzen [Hz] (quer) } & \multicolumn{3}{|c|}{ Eigenfrequenzen } \\
\hline & \multicolumn{3}{|c|}{$50 \mathrm{~m}^{3} / \mathrm{h}$} & \multicolumn{3}{|c|}{$110 \mathrm{~m}^{3} / \mathrm{h}$} & \multirow{2}{*}{$\begin{array}{c}f_{1} \\
{[\mathrm{~Hz}]}\end{array}$} & \multirow{2}{*}{$\begin{array}{c}\frac{f_{2}}{[\mathrm{~Hz}]} \\
\left.{ }^{2}\right]\end{array}$} & \multirow{2}{*}{$\begin{array}{c}\frac{f_{3}}{[\mathrm{~Hz}]} \\
\end{array}$} \\
\hline & $\mathrm{E}$ & Q & A & $\mathrm{E}$ & Q & A & & & \\
\hline 01-09 & 47,4 & 5,1 & 5,4 & 104,0 & 11,1 & 11,8 & $\underline{\underline{26,5}}$ & $\underline{72,7}$ & $\underline{106,3}$ \\
\hline $02-08$ & 50,0 & 7,4 & 10,4 & 110,0 & 16,3 & 22,9 & $\underline{26,5}$ & $\underline{72,7}$ & $\underline{106,3}$ \\
\hline 03-07 & 47,7 & 4,4 & 13,6 & 105,0 & 9,7 & 30,0 & $\underline{26,5}$ & $\underline{72,7}$ & $\underline{106,3}$ \\
\hline 04-03 & 4,4 & 15,0 & 13,7 & 9,7 & 33,0 & 30,2 & $\underline{26,5}$ & $\underline{72,7}$ & 106,3 \\
\hline $05-02$ & 5,9 & 14,8 & 15,5 & 13,0 & 32,6 & 34,0 & $\underline{\underline{70,0}}$ & 102,3 & 123,9 \\
\hline $05-04$ & 16,4 & 14,1 & 16,4 & 36,1 & 30,9 & 36,1 & $\underline{70,0}$ & 102,3 & 123,9 \\
\hline 05-09 & 46,0 & 10,5 & 18,8 & 101,0 & 23,1 & 41,3 & $\underline{\underline{70,0}}$ & $\underline{102,3}$ & $\underline{123,9}$ \\
\hline 13-09 & 42,3 & 11,7 & 18,5 & 93,0 & 25,7 & 40,6 & $\underline{\underline{70,0}}$ & $\underline{102,3}$ & 123,9 \\
\hline 14-03 & 20,8 & 8,7 & 12,1 & 45,8 & 19,1 & 26,7 & $\underline{26,5}$ & $\underline{72,7}$ & $\underline{106,3}$ \\
\hline 14-09 & 40,2 & 10,1 & 16,3 & 88,5 & 22,2 & 35,9 & $\underline{26,5}$ & 72,7 & $\underline{106,3}$ \\
\hline $15-06$ & 35,1 & 8,4 & 13,0 & 77,2 & 18,4 & 28,6 & $\underline{26,5}$ & $\underline{72,7}$ & $\underline{106,3}$ \\
\hline $17-09$ & 18,6 & 3,6 & 18,6 & 40,8 & 7,8 & 73,6 & $\underline{26,5}$ & $\underline{72,7}$ & $\underline{\underline{106,3}}$ \\
\hline
\end{tabular}

Tabelle 4-6: Einzelwirbelspektrum und Eigenfrequenzen des Rohrbündels BB1-L170 nach analytischer Rechnung. $\mathrm{E}=$ Einstromzone, $\mathrm{Q}=$ Querstromzone, $\mathrm{A}=$ Ausstromzone.

Die beschriebene Vorgehensweise erfordert aber einen großen Berechnungsaufwand. In Tabelle 4-6 sind daher als Beispiel auch nur die Rohre des Bohrbilds BB1-L170 mit ihren zugeordneten Anregungsspektren infolge Einzelwirbelanregung im Bereich 50 bis $110 \mathrm{~m}^{3} / \mathrm{h}$ Volumenstrom und den ersten drei Eigenfrequenzen dargestellt, die auch experimentell untersucht wurden. Eine Ei- 
genfrequenz, der eine Anregende bis $30 \%$ nahe kommt, ist unterstrichen, der nur die Doppelte einer Anregenden bis $30 \%$ nahe kommt, ist doppelt unterstrichen. Nach der ausführlichen analytischen Rechnung ist für die einfach gestützten Rohre von BB1-L170 mit steigendem Volumenstrom mit Lock-InVerhalten zu rechnen, während die doppelt gestützten Rohre weniger gefährdet sind. Zum einen sind diese Rohre steifer gelagert, zum anderen werden im mittleren Bereich des Wärmetauschers die geringsten Strömungsgeschwindigkeiten erreicht. Dadurch sind Eigenfrequenzen und Anregungsfrequenzen weit genug von einander entfernt. Diese analytischen Ergebnisse werden von den Meßergebnissen aus Kapitel 3 bestätigt.

\begin{tabular}{|c|c|c|c|c|c|c|c|}
\hline Bündel & $\begin{array}{c}f_{3} \\
{[\mathrm{~Hz}]}\end{array}$ & $\begin{array}{c}\tau \\
{[-]}\end{array}$ & $\begin{array}{c}m \Lambda /\left(\rho D^{2}\right) \\
{[-]}\end{array}$ & $\begin{array}{c}S r_{1} \\
{[-]}\end{array}$ & $\begin{array}{c}u_{s p, k r i t, 1} \\
{[\mathrm{~m} / \mathrm{s}]}\end{array}$ & $\begin{array}{c}S r_{2} \\
{[-]}\end{array}$ & $\begin{array}{c}u_{s p, k r i t, 2} \\
{[\mathrm{~m} / \mathrm{s}]}\end{array}$ \\
\hline BB1-L170 & 106,3 & 1,6 & 0,3937 & 0,12 & $\underline{\mathbf{7 , 4}}$ & - & - \\
\hline BB1-L220 & 106,3 & 1,6 & 0,5525 & 0,12 & 7,4 & - & - \\
\hline BB2-L170 & 107,4 & 1,6 & 0,4020 & 0,35 & $\underline{\mathbf{2 , 6}}$ & 0,51 & 1,8 \\
\hline BB2-L220 & 107,4 & 1,6 & 0,5629 & 0,35 & 2,6 & 0,51 & 1,8 \\
\hline BB3-L170 & 71,9 & 1,2 & 0,4921 & - & - & - & - \\
\hline BB3-L220 & 71,9 & 1,2 & 0,6997 & - & - & - & - \\
\hline BB4-L170 & 72,6 & 1,2 & 0,5096 & $(0,57)$ & $(1,4)$ & 0,80 & 1,0 \\
\hline BB4-L220 & 72,6 & 1,2 & 0,7217 & $(0,57)$ & $(1,4)$ & 0,80 & 1,0 \\
\hline
\end{tabular}

Tabelle 4-7: Lock-In-Gefährdung des Zentralrohrs der ersten Rohrreihe nach analytischer Rechnung [VDI97].

Zum anderen könnte man sich unter gewissen Voraussetzungen auch darauf beschränken, nur die Schwingungsgefährdung des Zentralrohrs der ersten Rohrreihe zu beurteilen. Hier treten die höchsten Spaltströmungsgeschwindigkeiten und damit auch die höchsten Wirbelablösefrequenzen auf, während gleichzeitig die Rohreigenfrequenzen am niedrigsten sind, da sie sich im Fensterbereich einiger Leitbleche befinden und damit weniger oft gestützt werden. Oft ist der Abstand zwischen Lock-In und kritischer Spaltgeschwindigkeit hinsichtlich fluidelastischer Instabilität auch so gering, daß es sich nicht weiter lohnt, ganze Spektren zu bestimmen, da ein Betrieb zwischen Lock-In und fluidelastischer Instabilität zu gefährlich wäre. In diesem Fall bleibt man mit der Anströmgeschwindigkeit unterhalb von $70 \%$ der niedrigsten Lock-In-Geschwindigkeit. 
Dieses Vorgehen ist konservativ, aber es ist weniger Berechnungsaufwand erforderlich. Die nach diesem Verfahren bestimmten Lock-In-Geschwindigkeiten sind in Tabelle 4-7 mit aufgeführt.

Lock-In tritt bei Flüssigkeiten dann nicht auf, wenn der Dämpfungsparameter $m \Lambda /\left(\rho D^{2}\right)>0,4$ und $\tau<1,5$ ist [VDI97]. Danach scheiden die Bündel BB3, BB4, BB1-L220 und BB2-L220 als Lock-In-gefährdet aus. Demnach können höchstens die Bündel BB1-L170 und BB2-L170 noch Lock-In infolge Einzelwirbelablösung erster Ordnung erleiden. Da sich in den Experimenten herausgestellt hatte, daß nicht die erste Eigenfrequenz angeregt wird, sondern die dritte, ist in Tabelle 4-7 die Berechnung der kritischen Spaltgeschwindigkeit unter Zugrundelegung der dritten Rohreigenfrequenz für das einfach gestützte Rohr durchgeführt worden. Die einfache Lagerung kann aktiv werden, da durch den herrschenden Staudruck an jedem Rohr sichergestellt ist, daß das Rohr wenigstens zeitweise den Lagerspalt überbrü cken kann.

\subsubsection{Halbempirische Kriterien gegen fluidelastische Instabilität}

Die Grenzspaltgeschwindigkeit zur fluidelastischen Instabilität wird mit Hilfe des erweiterten Connors-Kriteriums (1-1) [VDI97] und des erweiterten TroidlKriteriums (1-2) [Kas94] bestimmt. Die Schwingungen setzen bei den untersuchten Anordnungen in der ersten oder zweiten Rohrreihe zuerst ein, daher wird in konservativer Weise der Berechnungsvorgang für das Zentralrohr der ersten Rohrreihe durchgeführt.

Für die Anwendung beider Kriterien ist die Kenntnis des Belastungsfaktors $S$ für Schwingen in der ersten Eigenform nach (1-1) notwendig. Die erste Eigenform ergibt sich aus den analytischen Betrachtungen für das einfach asymmetrisch gestützte Rohr aus Abschnitt 3.3.4 und [Sto00], die normierte Geschwindigkeitsverteilung entlang der Längsachse des Rohres 01-09 aus den numerischen Simulationen mit CFX aus Abschnitt 4.2.2. Die Integralberechnung erfolgte numerisch. Die sich ergebenden kritischen Spaltgeschwindigkeiten sind in Tabelle 4-8 aufgeführt.

Nach [VDI97] ist der halbempirische Faktor $K$ im Connors-Kriterium auch vom Teilungsverhältnis $\tau$ abhängig, diese Abhängigkeit wird berücksichtigt. Das logarithmische Dekrement $\Lambda$ wurde ebenfalls gemäß [VDI97] berechnet. 


\subsection{Zusammenfassung und Methodenvergleich}

In Tabelle 4-8 sind die kritischen Spaltgeschwindigkeiten hinsichtlich fluidelastischer Instabilität und die Lock-In-Spaltgeschwindigkeit für die erste Rohrreihe aller acht betrachteten Rohrbündel nach den angeführten unterschiedlichen Bestimmungsmethoden angegeben. Die herrschenden Spaltgeschwindigkeiten bei $110 \mathrm{~m} / \mathrm{h}$ Volumenstrom betragen $10,4 \mathrm{~m} / \mathrm{s}$ für BB1 und $\mathrm{BB} 2$ sowie 23,6 $\mathrm{m} / \mathrm{s}$ für BB3 und BB4. Sie ergeben sich aus der Stutzengeschwindigkeit im Zulauf, die mit Hilfe der Kontinuitätsgleichung auf eine äquivalente Spaltgeschwindigkeit umgerechnet wird. Die in der Zusammenfassung angegebenen Werte für fluidelastische Instabilität wurden akustisch ermittelt. Für den LockIn wurde die kritische Geschwindigkeit durch Auswertung der RMSAmplitudenverläufe bestimmt.

\begin{tabular}{|c|c|c|c|c|c|c|}
\cline { 3 - 8 } \multicolumn{1}{c|}{} & \multicolumn{2}{c|}{ Fluidelastische Instabilität } & \multicolumn{2}{c|}{ Lock-In } \\
\cline { 2 - 8 } & $\begin{array}{c}\text { Faktor } S \\
\text { Gl. }(1-1) \\
{[-]}\end{array}$ & $\begin{array}{c}\text { Connors } \\
{[\text { [VI97] }} \\
{[\mathrm{m} / \mathrm{s}]}\end{array}$ & $\begin{array}{c}\text { Troidl } \\
{[\text { Kas94] }} \\
{[\mathrm{m} / \mathrm{s}]}\end{array}$ & $\begin{array}{c}\text { Versuch } \\
3.4 .1 \\
{[\mathrm{~m} / \mathrm{s}]}\end{array}$ & $\begin{array}{c}\text { Analytik } \\
{[\text { VDI97] }} \\
{[\mathrm{m} / \mathrm{s}]}\end{array}$ & $\begin{array}{c}\text { Versuch } \\
3.4 .2 \\
{[\mathrm{~m} / \mathrm{s}]}\end{array}$ \\
\hline BB1-L170 & 0,0117 & 7,4 & 9,8 & 11,1 & 7,4 & 8,8 \\
\hline BB1-L220 & 0,0091 & 5,7 & 6,4 & 7,3 & - & - \\
\hline BB2-L170 & 0,0086 & 10,6 & 9,7 & 19,5 & 2,6 & 2,8 \\
\hline BB2-L220 & 0,0053 & 9,3 & 7,2 & 16,2 & - & - \\
\hline BB3-L170 & 0,0138 & 7,6 & 9,1 & 41,4 & - & - \\
\hline BB3-L220 & 0,0079 & 6,8 & 6,9 & 25,1 & - & - \\
\hline BB4-L170 & 0,0070 & 7,7 & 12,3 & 18,0 & - & - \\
\hline BB4-L220 & 0,0053 & 6,1 & 8,2 & 37,5 & - & - \\
\hline
\end{tabular}

Tabelle 4-8: Kritische Spaltgeschwindigkeiten der Rohrbündel in der ersten Rohrreihe nach verschiedenen Kriterien.

Im Reihenversuch wird nur für BB1-L170 Lock-In erwartet, da die kritische Spaltgeschwindigkeit von 2,6 m/s für BB2-L170 entsprechend einem Volumenstrom von $27 \mathrm{~m}^{3} / \mathrm{h}$ noch weit unterhalb des gewählten Experimentierbereichs von 50 bis $110 \mathrm{~m}^{3} / \mathrm{h}$ liegt. Für den Einzelversuch mußte bei BB2-L170 der Absperrschieber (13) teilweise geschlossen werden, um den Förderbereich der Pumpe (2) entsprechend herabzusetzen (vgl. Abbildung 3-1). 
Wie aus Tabelle 4-8 ersichtlich ist, werden die Gefährdungen durch Lock-In mit Hilfe der analytischen Auslegung mit Unterschätzungen von $19 \%$ (BB1-L170) bzw. $8 \%$ (BB2-L170) recht gut vorhergesagt und liegen immer auf der sicheren Seite. Auch das Auftreten bzw. Nichtauftreten von Einzelwirbelanregung wird nach den Kriterien aus [VDI97] für alle Fälle korrekt vorhergesagt.

Anders verhält es sich bei der Absicherung gegen fluidelastische Instabilität. Auch die Anwendung der jüngsten analytischen Auslegungsrichtlinie im deutschsprachigen Raum [VDI97] führt für die gewählten praxisrelevanten Anordnungen in den meisten Fällen zu einer deutlichen Unterschätzung der kritischen Spaltgeschwindigkeit. Bei den im Rahmen der vorliegenden Arbeit durchgeführten Messungen lag der im Versuch bestimmte tatsächliche kritische Wert der Spaltgeschwindigkeit um einen Faktor von bis zu 6,15 höher als der nach [VDI97] und um einen Faktor bis zu 4,57 höher als der nach [Kas94] berechnete Wert (BB4-L220). Große Unterschätzungen ergaben sich dabei für BB3 und BB4, während die Unterschätzungen bei den Bündeln BB1 und BB2 geringer blieben.

Die Ergebnisse nach [VDI97] und [Kas94] lagen dabei immer auf der sicheren Seite. Demzufolge wird nach gängigen Methoden meistens deutlich zu konservativ ausgelegt. Dieses Ergebnis hatte auch Kassera in [VDI97a] für seine idealisiert einseitig eingespannten Rohrbündel erhalten. Das Resultat stellt die Hauptmotivation für die Entwicklung numerischer Simulationsverfahren wie in [Kas97a] und in der vorliegenden Arbeit dar.

\subsection{Ergebnisse aus numerischer Simulation}

\subsubsection{Auswahl des Beispielfalls}

Als Beispiel für die Anwendung des neu entwickelten Berechnungsverfahrens wird das Rohrbündel BB1-L170 in einer Simulation des Schwingungsverhaltens mit dem neu entwickelten Code FIVSIC-3D näher untersucht. Hinsichtlich der Auswahl dieses Bündels als Berechnungsbeispiel sind folgende Bemerkungen zu machen:

Die komplette Berechnung eines schwingenden Bündels hat sich als äußerst CPU-zeitaufwendig herausgestellt. Zum einen soll eine geometrisch ausreichende Auflösung gewählt werden, zum anderen verringert sich dann wegen des CFL-Kriteriums (2-91) auch der Zeitschritt entsprechend. Deshalb mußte 
ein Beispiel gewählt werden, das möglichst wenig Berechnungszeit in Anspruch nimmt.

Aus diesem Grunde scheiden zunächst alle Bündel mit der Länge 2,1 m aus, da hierfür ca. $30 \%$ mehr Speicherplatz und Rechenzeit benötigt würde als für die Bündel mit 1,6 m Länge. Des weiteren scheiden die Rohrbündel aus, bei denen der Rohrdurchmesser 16 mm beträgt, da hier die Versperrungen größer sind. Dadurch ergeben sich Spaltströmungsgeschwindigkeiten, die diejenigen bei Bündeln mit 12 mm-Rohren um etwa einen Faktor 2,2 übertreffen. Infolgedessen steigt die CFL-Zahl entsprechend an, und es sinkt der notwendige Zeitschritt, wodurch der Berechnungsaufwand wiederum um einen Faktor 2,2 ansteigt.

Es verbleiben die beiden Rohrbündel BB1-L170 und BB2-L170. Diese beiden Bündel sind außerdem die einzigen, bei denen nach analytischer Rechnung und experimentellem Befund auch eine resonante Einzelwirbelanregung erwartet werden konnte und die daher auch einen größeren physikalischen Aussagewert besitzen.

Gewählt wurde schließlich BB1-L170, da hier nach Experiment die geringere kritische Spaltgeschwindigkeit, etwa nur halb so groß wie bei BB2-L170, erwartet wird. Damit kann wegen des CFL-Kriteriums auch ein etwa doppelt so großer Zeitschritt gewählt werden, wodurch sich die Rechenzeit halbiert. Außerdem trat der Lock-In hier am deutlichsten hervor. Alle anderen Bündel können natürlich auch berechnet werden, allerdings bei deutlich höherem numerischen Aufwand.

\subsubsection{Vorgehen bei der Berechnung und Berechnungsparameter}

Angelehnt an die numerischen Ergebnisse aus Abschnitt 2.1.5 mußte für einen Rohrdurchmesser eine Auflösung von wenigstens 6 Zellen gewählt werden. In Rohrlängsachsenrichtung wurden 22 Berechnungsebenen gewählt. Insbesondere sind darin Ein- und Ausströmebene sowie die Leitblechebenen enthalten. Es ergibt sich eine Gesamtzellenzahl von 175 x 175 x $22=673750$ Zellen. Dabei konnte mit einem Streckungsverhältnis von ca. 1:36 das 1:20-Kriterium der LES-Simulation nach Abschnitt 2.4.4 nicht eingehalten werden.

Zunächst wurde, von einer Nullstartbelegung ausgehend, eine Startlösung generiert, indem ein Volumenstrom unterhalb der sich nach analytischen Methoden und Versuchsdaten ergebenden kritischen Größe eingestellt wurde. Für den numerischen Versuch wurden 70 m/h bzw. 100 m³/h gewählt. Die erwartete höch- 
ste Spaltgeschwindigkeit wurde nach Gleichung (2-93) zu $u_{s p}=9,5 \mathrm{~m} / \mathrm{s}$ abgeschätzt. Als Zeitschritt wurde $\Delta t=0,00005 \mathrm{~s}$ gewählt. Dies entspricht einer CFL-Zahl von 0,47, um für den überkritischen Bereich noch Reserven zu haben. Es mußten ca. 0,5 s reale Zeit simuliert werden, um den Beginn eines eingeschwungenen Zustands im gesamten System zu erreichen, dies entspricht 10000 Zeitschritten. Hierfür wurden bei einem Speicheraufwand von ca. 175 MByte RAM etwa 10 CPU-Wochen auf einem PC Pentium II $400 \mathrm{MHz}$ verbraucht, obgleich die gewählte Auflösung lediglich die absoluten Mindestanforderungen erfüllt. Wegen der Restriktionen aus dem zugrundeliegenden Forschungsvorhaben [Fis00a] konnte nicht auf einen Parallelrechner ausgewichen werden. Ein später eingesetzter neuerer Rechner vom Typ Pentium 4 1,5 GHz war etwa um einen Faktor 3,5 schneller. Weitere 3 CPU-Wochen waren notwendig, um auf der simulierten Startlösung aufsetzend einen eingeschwungenen Zustand (Abbildung 4-2) zu simulieren. Kassera [Kas97a] berichtet bei seinen zweidimensionalen Berechnungsbeispielen über eine ähnlich große Anzahl von Zeitschritten, die notwendig war, um sein System in einen eingeschwungenen $\mathrm{Zu}-$ stand zu bringen.

Danach wurde der Volumenstrom über eine Rampenfunktion auf einen Wert oberhalb der kritischen Anströmgeschwindigkeit entsprechend einem kritischen Volumenstrom von ca. $117 \mathrm{~m}^{3} / \mathrm{h}$ erhöht. Im numerischen Versuch wurden hierfür $140 \mathrm{~m}^{3} / \mathrm{h}$ gewählt.

\subsubsection{Beschreibung der Simulationsergebnisse}

In Abbildung 7-7 ist die sich dabei einstellende Geschwindigkeitsverteilung in der Zuströmebene des Wärmetauschermodells im eingeschwungenen Zustand bei $100 \mathrm{~m} / \mathrm{h}$ dargestellt. Jede Strömungszelle ist dabei entsprechend ihrer Belegung farblich markiert. Deutlich erkennbar sind die Gassenströmungen im Zentralbereich des Modells. Ein solches Verhalten würde man auch anschaulich erwarten. Überraschender ist hingegen der relativ hohe Massenstrom, der sich durch Gassenbildung zwischen Behältermantel und den Rohren 1 bzw. 5 (entsprechend 01-07 bzw. 01-11 nach experimenteller Notation) ergibt. Diese Massenströme werden nochmals an den Rohren 6 bzw. 14 (entsprechend 02-05 bzw. 02-13 nach experimenteller Notation) in einen großen Anteil, der zwischen Rohrbündel und Behältermantel vorbeifließt, und einen kleinen Anteil, der eine weitere Gassenströmung im Zentralbereich des Modells hervorruft, aufgeteilt. Dies entspricht auch den Resultaten aus [Fis98b, Fis98c]. Bei dem 
dort numerisch untersuchten Gaskühler waren Leitbleche notwendig, um die Strömung auf ihren druckverlustreicheren Weg durch das Rohrbündel zu zwingen. Wie im vorliegenden Beispiel sucht sich die Strömung sonst aus energetischen Gründen den leichteren Weg am Bündel vorbei.

In Abbildung 7-8 ist die Strömung am Eintritt detailliert dargestellt. Deutlich sind die Totwassergebiete hinter den Rohren zu sehen. Die Vektorverteilungen deuten die Wirbelbildung hinter den Hindernissen an. Auch alle Jet-Bildungen sind deutlich zu erkennen.

In Abbildung 7-9 ist die Druckverteilung in der Einströmebene dargestellt. Hoher Druck entspricht dabei Staupunktbereichen, die vor den angeströmten Rohren zu sehen sind. Hinter den Rohren erkennt man Tiefdruckgebiete, die auf Totwassergebiete und Wirbelablösungen schließen lassen. Auffällig sind die beiden relativ deutlichen Staupunkte der Rohre 6 und 14 (bzw. 02-05 und 02-11 nach experimenteller Notation) sowie die im Randbereich fortschwimmenden abgelösten Wirbel. Im hinteren Bereich staut sich die Strömung wieder auf und bildet Wirbelsysteme. Im Zentralbereich ergeben sich entlang der Strömungsgassen immer wieder Tiefdruckgebiete im Ablösebereich der Rohre, die ursächlich für die instationären Strömungskräfte an den Rohren sind.

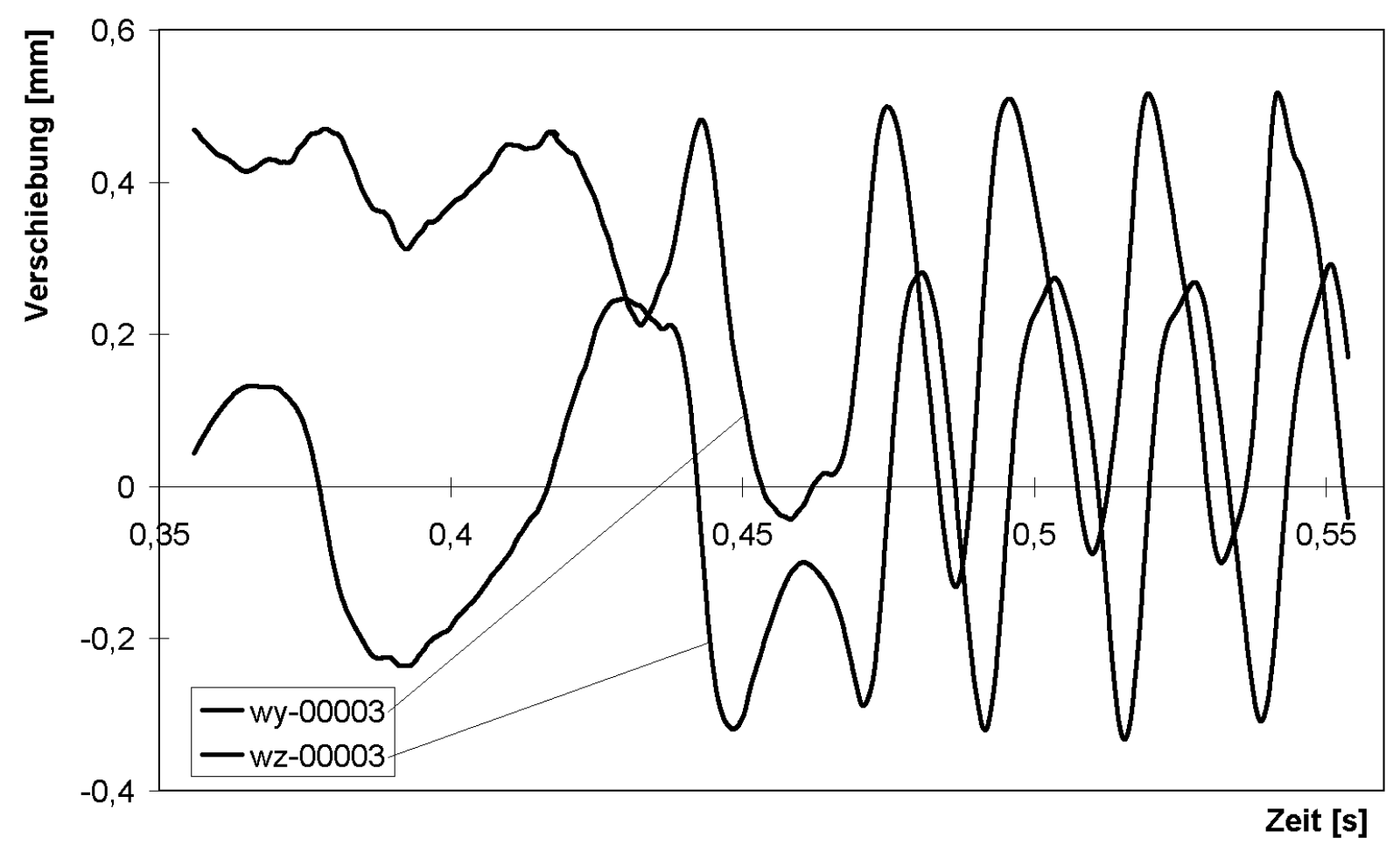

Abbildung 4-2: Verschiebungen von Rohr BB1-L170-T1423-R0109 bei $100 \mathrm{~m}^{3} / \mathrm{h}$ Nennvolumenstrom. 
In Abbildung 7-10 ist der Einströmbereich nochmals detailliert dargestellt. Dabei erkennt man, wie die Wirbelbildung, dargestellt durch die Strömungsvektoren je Strömungszelle, mit den jeweiligen Tiefdruckgebieten korrespondieren. Eine Erhöhung der Strömungsgeschwindigkeit in den Gassen geht immer mit einer Druckabsenkung entsprechend dem Prinzip der Energieerhaltung einher.

Abbildung 7-11 zeigt Druck- und Geschwindigkeitsverteilung im dreidimensional dargestellten Gesamtmodell. $\mathrm{Zu}$ erkennen sind Zonen hoher und niedriger Strömungsgeschwindigkeit und der Gesamtdruckverlust vom Eintritt zum Austritt des Systems. In den Fenstern der Leitbleche ergeben sich erhöhte Strömungsgeschwindigkeiten, da hier der Strömungsquerschnitt verringert wird.

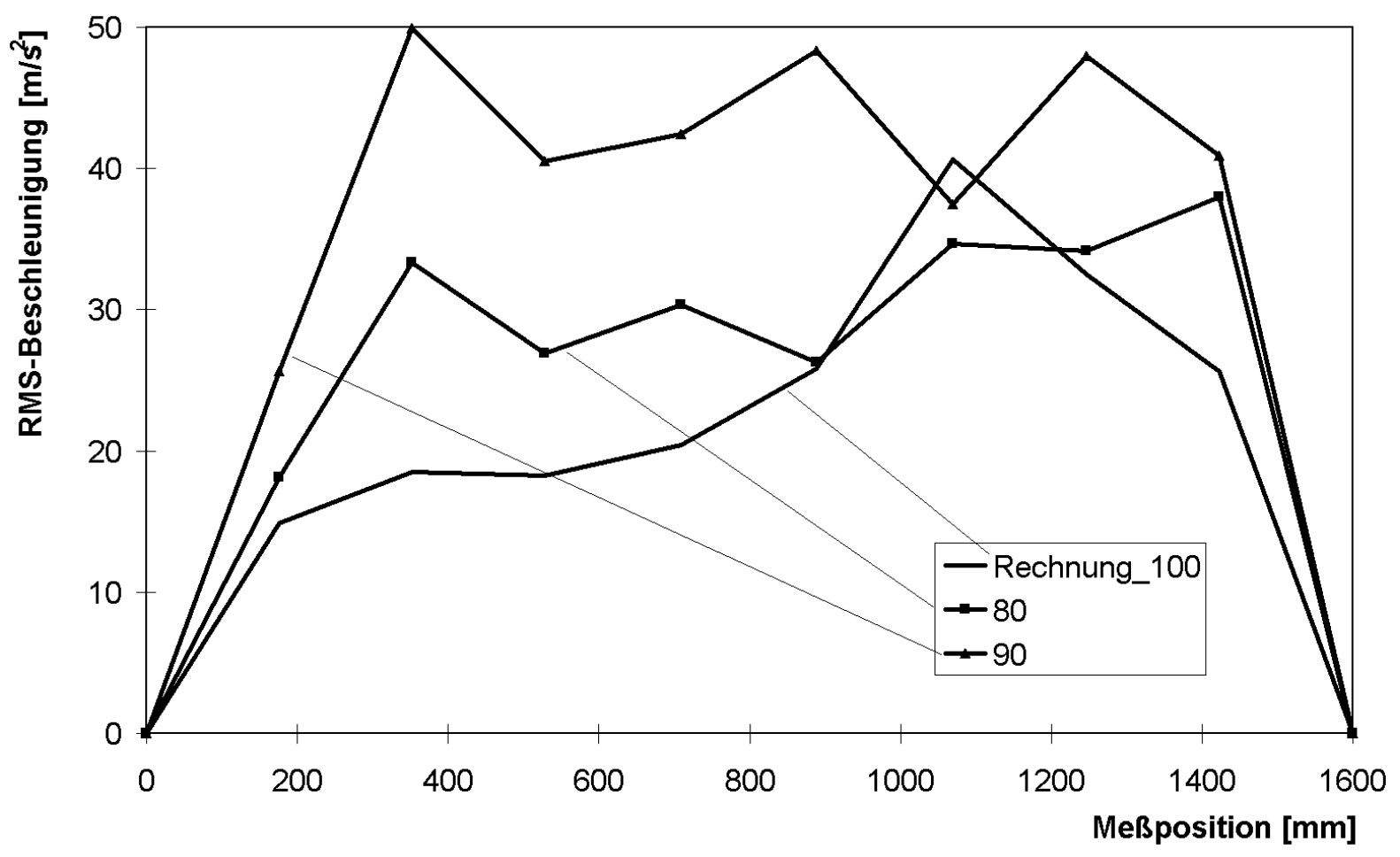

Abbildung 4-3: RMS-Verteilung bei Rohr BB1-L170-T1423-R0403 in Experiment und Rechnung bei Nennvolumenstrom $100 \mathrm{~m}^{3} / \mathrm{h}$.

Die Farbdarstellungen zeigen, daß die entscheidenden Strömungsphänomene qualitativ richtig simuliert werden. Abbildung 4-2 zeigt den eingeschwungenen zeitlichen Verlauf der Verschiebungen des Rohrs 3 (bzw. 01-09) auf Stutzenhöhe in Strömungsrichtung und quer dazu. Die Querströmungskomponente schwingt um den Wert Null herum, während die Verschiebungskomponente in Strömungsrichtung um einen Offset-Wert herum schwingt, so, wie es vom Einzelzylinder her zu erwarten ist. Allerdings ergeben sich infolge der heftigen 
Schwingungsbewegungen auch Anteile entgegen der Nullage. Dies kann daran erkannt werden, daß der Verlauf der Verschiebungen in $y$-Richtung zeitweise negativ wird. Die gleichbleibenden Spitzenamplituden deuten an, daß es sich tatsächlich um den eingeschwungenen Zustand handelt. Ab $140 \mathrm{~m}^{3} / \mathrm{h}$ ergaben sich im Bereich der Fenster große Auslenkungen entsprechend beginnendem instabilem Verhalten. Dieser Wert ist im Vergleich zu $117 \mathrm{~m}^{3} / \mathrm{h}$ im Versuch etwas zu hoch und mag mit den bei der aufgrund der notwendigen Rechenleistung etwas zu groben Auflösung mit zu geringen Auftriebsbeiwerten entsprechend Abbildung 2-6 zusammenhängen.

Um mit experimentellen Ergebnissen vergleichen zu können, werden die RMSVerteilungen der Rohrbeschleunigung entlang der Rohrlängsachse und die jeweils zugehörige charakteristische Fouriertransformierte aus Messung und Rechnung gegenübergestellt. Als Beispiel hierfür sei in Abbildung 4-3 das Rohr 26 bzw. 04-03 betrachtet. Der Verlauf der berechneten RMS-Werte stimmt mit dem Verlauf der Meßwerte qualitativ gut überein, allerdings fällt das Niveau, ebenfalls wohl wegen zu niedriger Auftriebsbeiwerte, dabei etwas zu gering aus. Es werden bei $100 \mathrm{~m}^{3} / \mathrm{h}$ simuliertem Volumenstrom etwa die RMS-Werte für die Messungen zwischen 80 und $90 \mathrm{~m} 3 / \mathrm{h}$ erreicht.

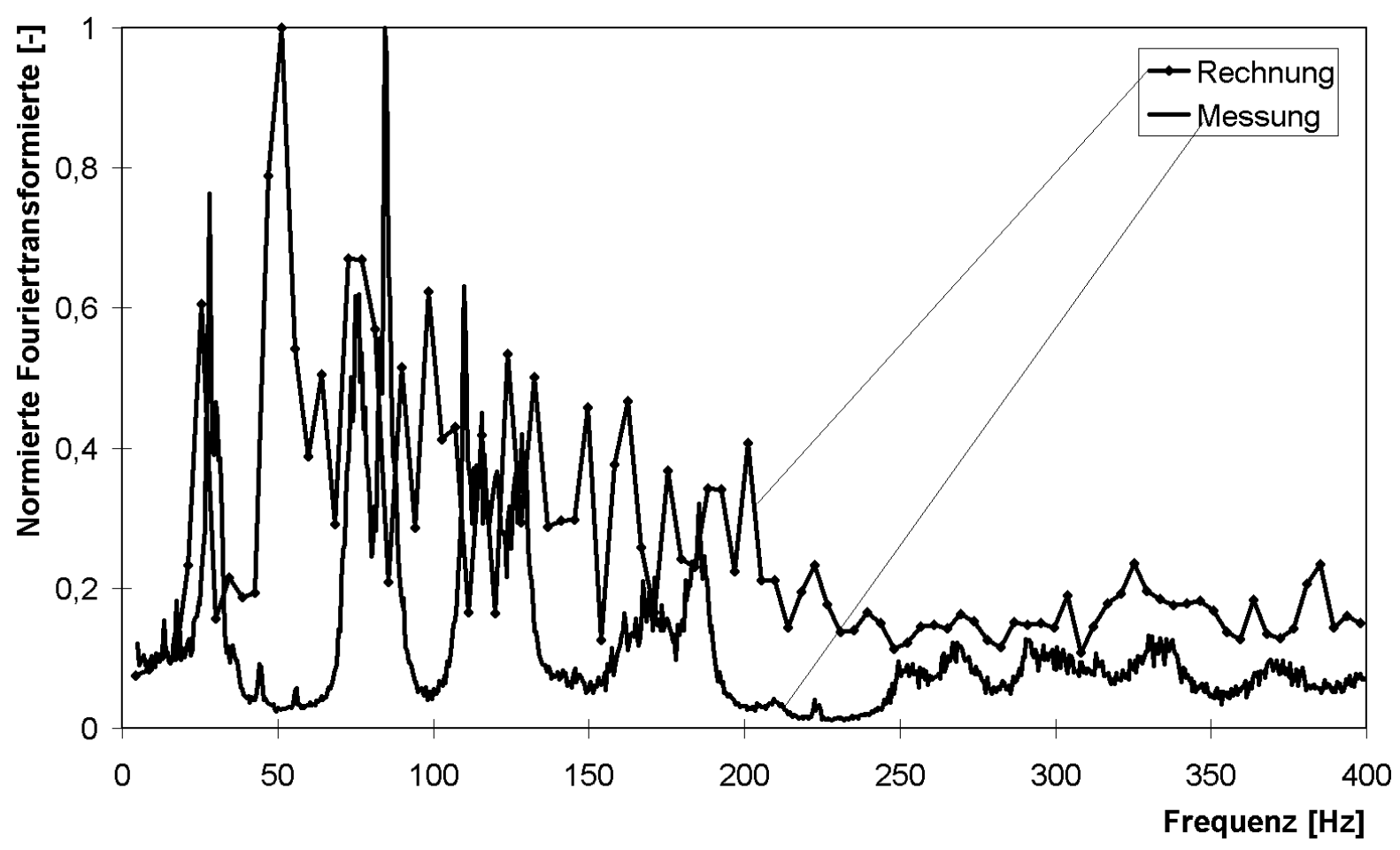

Abbildung 4-4: Charakteristische Fouriertransformierte bei Rohr BB1-L170T1423-R0403 in Experiment und Rechnung bei Nennvolumenstrom 100 m³. 
In Abbildung 4-4 ist die zugehörige charakteristische Fourier-Transformierte in normierter Darstellung zu sehen. Der Verlauf der Spektren stimmt gut überein. In der Messung treten die Peaks der Eigenfrequenzen schärfer hervor als in der Simulation. Dabei ist zu beachten, daß erstens aufgrund der kürzeren Simulationszeit, Gleichung (3-7) folgend, die Frequenzauflösung schlechter sein muß als in der längeren Messung. Zweitens gehen weniger Schwingungen in die Auswertung mit ein, so daß der Rauschanteil stärker hervortritt.

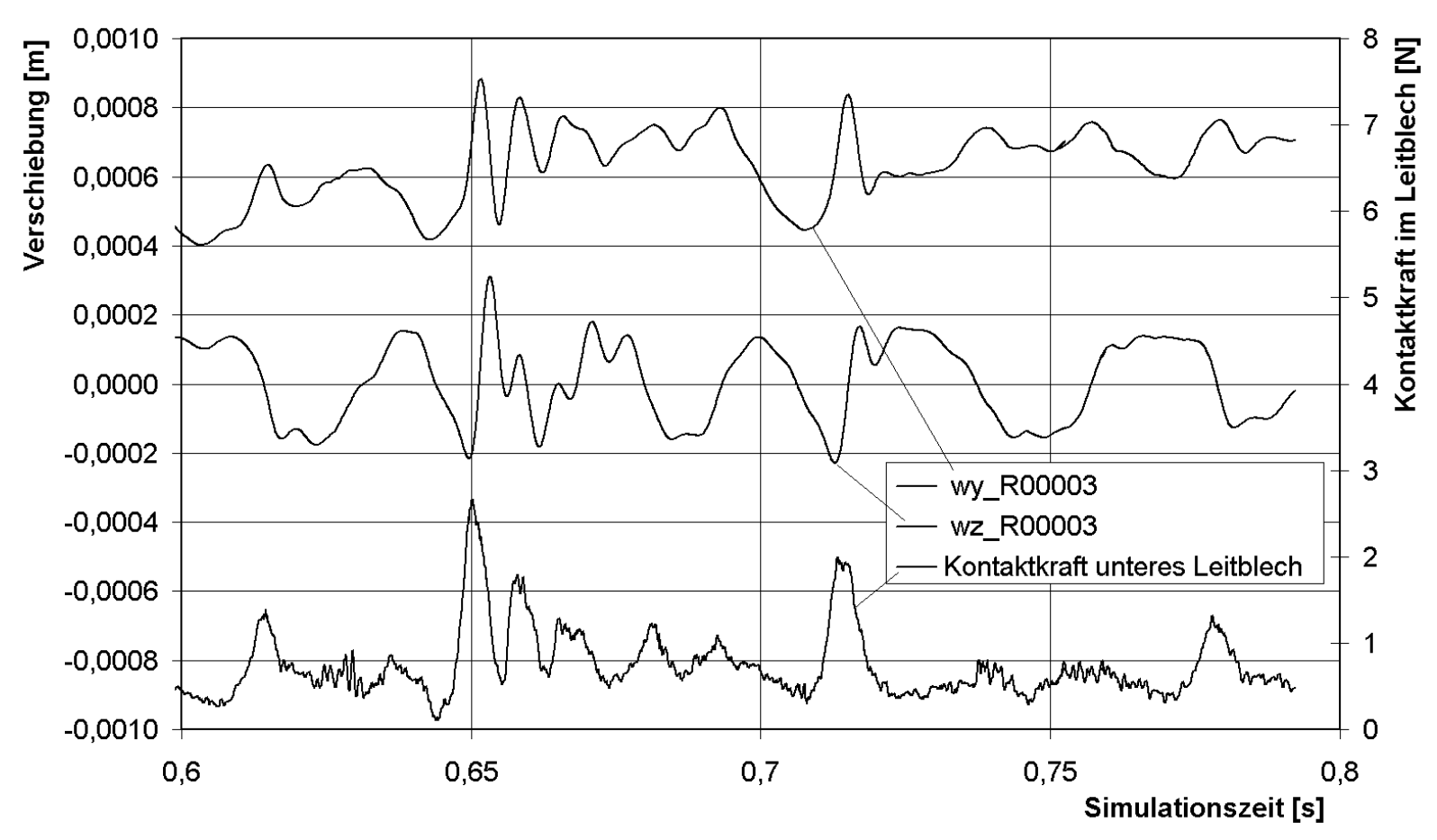

Abbildung 4-5: Anregung höherer Eigenfrequenzen infolge Lagerkontakt.

Kontaktkraft Rohr BB1-L170-R0109 und Verschiebungen auf Stutzenh öhe.

In Abbildung 4-5 ist schließlich der Einfluß eines plötzlichen harten Stoßes zwischen Rohr und Lagerblech bei $t=0,65 \mathrm{~s}$ am Beispiel des Rohrs BB1-L170R0109 dargestellt. Das Rohr schwingt zunächst mit der Frequenz der ersten Eigenform. Aufgrund einer plötzlich signifikant höheren Kontaktkraft zwischen Rohr und Leitblech wird das Rohr angeregt, in der dritten Eigenform zu schwingen. Klingt der Einfluß des Stoßes ab, so beruhigt sich das Rohr wieder und schwingt wieder in der ersten Eigenform. Je höher die Stoßkraft ist, desto deutlicher tritt der Effekt der Anregung der höheren Eigenfrequenzen hervor. Dies ist ein wesentlicher Effekt, der bei Anwendung Connors-ähnlicher Stabilitätskriterien [Con78, VDI97] nicht berücksichtigt werden kann. 


\section{Zusammenfassung und Ausblick}

\subsection{Zusammenfassung der Arbeitsinhalte}

In der vorliegenden Arbeit wurde ein numerisches Simulationsverfahren entwickelt, das auf Basis der Grundgleichungen für Strömungsmechanik und Rohrbündeldynamik gekoppelte Rohrbündelschwingungen berechnen kann.

Die Erstellung des Programms war ein Hauptteil der Arbeiten. Es können Geradrohrbündelwärmetauscher mit Leitblechen und Leitblechspalten sowie $\mathrm{Zu}$ und Abströmstutzen modelliert werden. Der Kontakt zwischen Rohren und Leitblechen ist in der Simulation enthalten. Die Strömungsphänomene am Spalt werden analytisch erfaßt. Das dynamische Modell wurde anhand analytischer Formeln für Eigenfrequenzen und statische Durchbiegungen validiert. Der strömungsmechanische Teil wurde anhand von Literaturangaben über Widerstand und Auftrieb am Einzelzylinder überprüft. Die Strömungssimulation ist in Raum und Zeit von zweiter Ordnung genau. Es wird ein dynamisches LESTurbulenzmodell verwendet. Die Kopplung erfolgt über ein kartesisches Gitter, das vom Zellüberdeckungsgrad abhängig ausblockiert wird. Übergaberandbedingungen sind Strömungsgeschwindigkeiten, Rohrpositionen, Rohrgeschwindigkeiten und Strömungskräfte. Besondere Aufmerksamkeit wurde der Aufgabe der koppelnden Randbedingungen während der Iteration der Zeitschritte gewidmet, wodurch ein sehr stabiler Code entstand. Der Kontakt im Leitblech stellt sich auch für den normalen Betriebsfall als schwingungsdominierend heraus. Der Kontakt zwischen den Rohren konnte wegen des stabilen Programmverhaltens ebenfalls implementiert werden. Dadurch sind auch extreme Strömungssitiationen abgedeckt. Es wurde außerdem eine Reihe einzuhaltender Empfehlungen zur Stabilisierung des Verfahrens erarbeitet.

Als zweiter Hauptteil der Arbeiten wurde zur Überprüfung der Rechenergebnisse ein Prüfstand erstellt, an dem ca. 16000 Zeitreihen der Beschleunigungen der Rohre gemessen wurden. Für die piezoelektrischen Beschleunigungssensoren wurde eine spezielle Aufnahmevorrichtung entwickelt. Die Beschleunigungszeitreihen werden mit Fourier-Analyse und RMS-Berechnungen ausgewertet. Diese Analysen gestatten die Lokalisierung stark beanspruchter Zonen des Rohrs sowie die Ermittlung der maßgeblichen Anregungsmechanismen. Die Übereinstimmung der RMS-Analysen der Zeitreihen mit den vom Programm errechneten RMS-Werten eines Testrohrbündels sind zufriedenstellend. 


\subsection{Weiterentwicklungen gegenüber Kassera}

Kassera [Kas97a] gelang es erstmals, fluid-struktur-gekoppelte Rohrbündelschwingungen unter Berücksichtigung der Turbulenz numerisch zweidimensional zu simulieren. Seine Arbeit war demzufolge auch der Ausgangspunkt der vorliegenden Arbeit, obwohl der hier verwendete Code wegen der Nichterweiterbarkeit von Kasseras Programm auf drei Dimensionen von Grund auf neu entwickelt werden mußte [Fis00a]. Gegenüber der Arbeit von Kassera mußten eine Reihe von Verbesserungen eingeführt werden, die zumeist in der umfassenderen dreidimensionalen Problematik der vorliegenden Arbeit begründet waren.

Aufgrund des nur zweidimensional und zwischenlagerfrei angenommenen Problems konnte Kassera mit der Erfassung der ersten Eigenfrequenz auskommen, sowohl beim Experiment als auch in der Simulation. Dadurch erhielt er ein sehr einfaches, auf einem Feder-Masse-Schwinger beruhendes Berechnungsmodell für die Rohrschwingungen. Im dreidimensionalen Fall haben die realen Zwischenlager in den Leitblechen jedoch entscheidenden Einfluß auf das Schwingungsverhalten der Rohre. Die Lagerungen der im Versuch verwendeten Bündel bei ${ }^{1} / 3 L_{0}$ und ${ }^{2}{ }_{3} L_{0}$ führten oft zu einer dominanten Anregung der dritten und sechsten Eigenfrequenz, die erste Eigenfrequenz trat nie so deutlich hervor wie diese höheren Eigenfrequenzen. Dies hatte zur Folge, daß für die Simulation ein aufwendiges Finite-Differenzen-Modell für die Rohrdynamik implementiert werden mußte, bei dem auch das Kontaktproblem zwischen Rohren und Leitblechen Berücksichtigung fand. Für die Abdeckung von Grenzfällen der Simulation war außerdem die Implementierung eines Kontaktmodells für das Anschlagen von Rohren untereinander notwendig. Die Nichtberücksichtigung dieses Falles hätte in Grenzfällen das Programm zum Absturz bringen können. Diese Zusammenhänge erforderten ein besonders robustes Berechnungsmodell für die Strukturdynamik.

Aus demselben Grund mußte auch die Art und Weise der Iteration der Zeitschritte verbessert werden. Während Kassera gute Ergebnisse damit erzielen konnte, daß nur zum Ende eines bei fest angenommener Rohrbündelgeometrie ausiterierten Strömungszeitschrittes die neue Lage der Rohre aufgrund der Strömungskräfte am Ende des Zeitschrittes berechnet wurde, mußten in der vorliegenden Arbeit die neue Rohrlage und die neuen Rohrgeschwindigkeiten zum Ende jeder Iteration eines Strömungszeitschritts berechnet werden, um Konvergenz und Stabilität zu erreichen. Der Grund liegt möglicherweise darin, 
daß die Rohrgeschwindigkeiten ja eine innere Randbedingung für das Strömungsfeld in der Umgebung der Rohre darstellen und somit die Berechnung der Rohrgeschwindigkeiten auch als Teil der Berechnung der Strömungsgeschwindigkeiten betrachtet werden muß. Da die Auslenkungen und Geschwindigkeiten der Rohre in drei Dimensionen durch eine Anzahl von Knoten dargestellt werden mußten, mußte auch das Subsystem der Rohrbündelstruktur iterativ gelöst werden. Für die Aufgabe der Strömungskräfte nach jeder Strömungsiteration war dabei die Einführung von Rampenfunktionen notwendig, um Stabilität und Konvergenz zu erreichen. Gegenüber der Arbeit von Kassera konnten so auch etwas größere Zeitschritte verwendet werden. Allein durch den Übergang zu drei Dimensionen stieg also schon der Aufwand für Strukturberechnung und Kopplung stark an und erforderte die Anwendung eines erweiterten Grundlagenwissens.

Mit Kasseras zweidimensionaler Rechnung konnten lediglich homogen angeströmte Rohrbündel simuliert werden, den Rohren wurde dabei nur eine dominierende Eigenfrequenz zugestanden. Für den Übergang zu realen Apparaten war eine dreidimensionale Simulation der Strömung notwendig. Nur so kann der Einfluß von Stutzen, Leitblechen, Spalten und realer Strömungsführung richtig erfaßt werden, so daß die Rohrbündel überall mit den richtigen Spaltgeschwindigkeiten durchströmt werden. Die Spaltgeschwindigkeiten sind die entscheidende Größe für die Schwingungsanregung der Rohrstruktur. Außerdem ist die Turbulenz der Strömung grundsätzlich immer ein dreidimensionales Phänomen, ihre Simulation in zwei Dimensionen kann methodisch kritisiert werden. Zudem wird durch eine zweidimensionale Rechnung die Korrelationslänge der ablösenden Wirbel nicht erfaßt. In [VDI97] wird vorgeschlagen, die Korrelationslänge mit etwa dem achtfachen Wert des Rohrdurchmessers abzuschätzen. Dieser Wert ist jedoch wesentlich kleiner als die im Versuch verwendeten Rohrlängen. Im dreidimensionalen Fall sind daher gegenüber dem zweidimensionalen Modell reduzierte Kräfte zu erwarten.

Da sich der Rechenaufwand in drei Dimensionen stark gegenüber dem ebenen Modell erhöht, waren auch auf der Seite der numerischen Strömungsmechanik eine Reihe von Verbesserungen notwendig, um die Rechengeschwindigkeit zu erhöhen.

Hinsichtlich der in der Vorgängerarbeit verwendeten mit den Rohrbewegungen mitverzerrten bzw. mitbewegten Subgittern der Rohre berichtet Kassera von immer wieder aufgetretenen Instabilitäten, die er auf die notwendigen Interpo- 
lationen zwischen seinen Grund- und Rohrgittern zurückführte [Kas98]. Möglicherweise waren hier die numerische Diskretisierung der konvektiven Terme mit QUICK als Verfahren zweiter Ordnung und die nur linear durchgeführte Interpolation nicht konsistent und es hätten die Instabilitäten zumindest bei den mitbewegten Gittern durch eine ebenfalls mit zweiter Ordnung durchgeführte Interpolation behoben werden können. Dies hätte jedoch einen weiteren Aufwand bedeutet. Für die verzerrten Gitter können die Instabilitäten bei größerer Verformung möglicherweise auf zu große Seitenlängenverhältnisse zurückgeführt werden. Außerdem wird in der Arbeit von Kassera das Space Conservation Law [Dem88, Dem90] nicht explizit berücksichtigt. Kassera hatte bereits Versuche unternommen, Rohre durch blockierte Zellen im Grundgitter darzustellen. Dabei wurden die Zellen freigegeben, die vom Rohrquerschnitt aufgrund seiner Bewegung zu nicht mehr als $50 \%$ überdeckt wurden. Auch hier ergaben sich Instabilitäten aufgrund der Nichtlinearitäten durch das sich in Grenzfällen ständig ergebende Umschalten zwischen Blockade und freier Strömung [Kas98]. Um diese Instabilitäten vollständig zu vermeiden, wurde in der vorliegenden Arbeit das Konzept der teilblockierten Zellen eingeführt. Dadurch wird das Problem der Zellblockaden linearisiert. Hierfür war eine umfangreiche Unterroutine zur Berechnung des Anteils der Blockade jeder Strömungszelle notwendig. Instabilitäten aufgrund des numerischen Gitters und der Rohrbewegung konnten damit vollständig vermieden werden. Die Anwendung des Space Conservation Laws ist nicht mehr notwendig, die Verwendung aufwendiger vollständig körperangepaßter Gitter, die auch bei der Annährung von Rohren große Nachteile gehabt hätten, und auch die aufwendige Benutzung unstrukturierter Gitter konnten so umgangen werden.

Um eine höhere Genauigkeit zu erreichen, verwendete Kassera das QUICKVerfahren zur Diskretisierung der konvektiven Terme der Impulsgleichungen. Aufgrund unphysikalischer Überschwinger bei der Interpolation zweiter Ordnung zur Berechnung der konvektiven Größen auf den Zellflächen mußte er jedoch mit dem UPWIND-Verfahren erster Ordnung gewichten, um Stabilität zu erreichen. Dies erforderte eine Reihe von Variationsrechnungen, um den optimalen Gewichtungsfaktor zu ermitteln, da die Ergebnisse der Rechnung hiervon abhängig waren. Zur Auflösung der relevanten Strömungsphänomene, insbesondere der Wirbelablösung, ist jedoch ein Verfahren zweiter Ordnung unbedingt notwendig. In der vorliegenden Arbeit wurden daher hochauflösende Ansätze getestet, wie z.B. MLU und COPLA. Hochauflösende Verfahren unter- 
drücken unphysikalische Überschwinger. MLU hat sich dabei als sehr robust und wirtschaftlich herausgestellt. Auch die zeitliche Diskretisierung, die bei Kassera noch erster Ordnung war, wurde im vorliegenden Verfahren zweiter Ordnung durchgeführt. International wird inzwischen empfohlen, bei strömungsmechanischen Berechnungen aufgrund der wesentlich besseren Genauigkeit immer mindestens zweiter Ordnung zu diskretisieren [Leo95].

Hinsichtlich des Turbulenzmodells konnte Kassera mit einem Standard-LESAnsatz gute Ergebnisse erzielen, jedoch war die Vorgabe einer Modellkonstanten notwendig. Hierdurch ergab sich wiederum die Notwendigkeit, den optimalen Parameter durch Variationsrechnungen zu ermitteln. Kassera ging daraufhin zum $k$ - $\omega$-Modell über. Dies hatte jedoch den Nachteil, daß zwei weitere Transportgleichungen gelöst werden mußten. In der vorliegenden Arbeit wurde daher der LES-Ansatz um die lokal optimierte Berechnung der LES-Konstanten nach Lilly und Germano erweitert. Es müssen daher keine weiteren Gleichungen iteriert werden und wie beim $k$ - $\omega$-Modell darf ohne Verwendung von Randfunktionen durch die Grenzschicht hindurchintegriert werden. Außerdem ist das Lilly-Germano-LES-Modell im Gegensatz zum $k$ - $\omega$-Modell und zum StandardLES-Ansatz in der Lage, ,backscatter“ zu berücksichtigen.

Bei der Berechnung des Druckfeldes verwendete Kassera den SIMPLEAlgorithmus zur Druckkorrektur, jedoch war eine Unterrelaxation erforderlich. Dadurch wird eine weitere numerische Größe eingeführt. In der vorliegenden Arbeit wurde daher SIMPLEC verwendet. Die Unterrelaxation kann dabei entfallen, außerdem wird weniger Rechenzeit und Speicherplatz benötigt. Die Konvergenzrate hat sich durch Anwendung von SIMPLEC verdoppelt. Um die Wärmetauschermodelle in Rohrlängsachsenrichtung stark strecken und das Konzept der teilblockierten Zellen durchgängig verwenden zu können, mußten besondere Vorgehensweisen für die Lösung der Druckkorrekturgleichung entwickelt werden. Zur Lösung der sowohl beim Druckkorrekturverfahren als auch bei der Lösung der Impulsgleichungen entstehenden linearisierten Gleichungssysteme verwendete Kassera einen Gauss-Seidel-point-by-point-Solver. Inzwischen sind jedoch eine Reihe leistungsfähigerer Verfahren bekannt. In der vorliegenden Arbeit wurden daher ADI, SIP3D, ICCG und CGSTAB implementiert. Durch sinnvolle Wahl der Solver konnte die Konvergenzrate gegenüber dem einfachen Solver von Kassera versechsfacht werden. 


\subsection{Vorteile der Simulation gegenüber bisherigen Verfahren}

Das in Fortsetzung von [Kas97a] erarbeitete Verfahren zur Beurteilung von strömungsinduzierten Schwingungen in Rohrbündelwärmetauschern stellt für den wissenschaftlich-technischen Fortschritt im Vergleich zu bisher verwendeten halbempirischen Auslegungskriterien [Con78, Kas94, VDI97] insgesamt einen methodischen Neubeginn dar. Bisher konnte lediglich die Einzelwirbelablösungsfrequenz und die kritische Anströmgeschwindigkeit hinsichtlich fluidelastischer Instabilität, diese jedoch mit einem Fehler bis $500 \%$ [Kas97a], halbempirisch vorhergesagt werden.

Darüber hinaus ist inzwischen ein schwerer Schadensfall infolge fluidelastischer Instabilität bekannt [Fis02], der auch bei Anwendung der Richtlinie [VDI97] nicht zu erklären war. Einige der bekannten Meßpunkte von Weaver [Wea82, Wea83] für Rohrbündel in 60-Grad-Anordnung und bei niedrigem Dämpfungsparameter $\Delta<0,3$ sind nicht in die Auslegungsformeln der Richtlinie [VDI97] eingeflossen, so daß die kritischen Spaltgeschwindigkeiten für diesen Bereich zu hoch vorhergesagt wurden. Dies führte zu einer weiteren Modifikation des Connors-Kriteriums, bei der die Stabilitätskonstante schließlich auf 1 gesetzt wurde und für Dämpfungsparameter $\Delta<0,3$ von einer völligen Unabhängigkeit der kritischen Spaltgeschwindigkeit von der Dämpfung ausgegangen wird [Fis02]. [VDI97] ist daher nicht in allen Fällen konservativ, in einigen Fällen führt auch diese Auslegungsmethode zu schweren Schadensfällen. In der internationalen Literatur wird die Allgemeingültigkeit der Connors-artigen Kriterien inzwischen bestritten [Pri01].

Vom wissenschaftstheoretischen Standpunkt aus ist bei Connors-artigen Kriterien vor allem zu bemängeln, daß sie lediglich deskriptiven Charakter haben, während sich numerische Simulation um eine explikative Untersuchung der Phänomene bemüht. So ist es auch zu erklären, daß in der Vergangenheit das ursprüngliche Connors-Kriterium [Con78] immer wieder korrigiert werden mußte, je nachdem, welche neuen Meßwerte bekannt wurden.

Mittels numerischer Simulation ist es über die genauere Bestimmung der kritischen Werte hinaus möglich, Aussagen über die Schwingungsamplituden der Rohre zu erhalten, damit auf das herrschende Spannungsniveau zu schließen und somit letztlich über Schadensakkumulationshypothesen Lebensdauerwerte für die Rohre anzugeben [Rom98]. Über die Auswertung der Kontaktkräfte in den Spalten zwischen Rohren und Leitblechen ist weiter der Weg zu einer Be- 
wertung des Abriebs der Rohre in den Leitblechen geebnet. Außerdem ist jede strömungsmechanische bzw. strukturdynamische Größe an jedem Ort und zu jeder Zeit verfügbar. Weitere den Wärmetauscher charakterisierende strömungstechnische Werte, wie etwa den Druckverlust des Wärmetauschers, erhält man zusätzlich zur Schwingungsanalyse quasi als Nebenprodukt.

\subsection{Vorteile gegenüber kommerziellen Simulationscodes}

Kommerzielle Programme zur Simulation von Strömungen, Strukturmechanik und gekoppelten Problemen erheben den Anspruch, leicht zu bedienen zu sein und möglichst für eine Vielzahl von Problemklassen verwendbar zu sein. Dies erfordert, daß eine genaue Diskretisierung der physikalischen Grundgleichungen vorgenommen wird und die numerische Lösung mit einem allgemeingültigen Solver berechnet wird. Für die vorliegende Aufgabe ergeben sich damit folgende Probleme:

- Für die Berechnung des Kontaktes zwischen einem schwingenden Rohr und einem Leitblech ist ein äußerst feiner Zeitschritt erforderlich, da sich die Lösungsmatrizen der hierfür verwendeten Softwarecodes ,nicht gutartig“ verhalten in bezug auf die Kopplung der Schwingungsbewegung in die zwei Raumrichtungen. Der Kontakt führt zu einer Kopplung der beiden Bewegungen. Im vorliegenden Programm konnte hierfür eine spezielle Routine entwickelt werden, die lokal eine gekoppelte Lösung erlaubt.

- Seitenkantenverhältnisse von mehr als 1:2 werden für numerische Zellen in Strömungsproblemen nicht empfohlen. Der Grund dafür liegt in der schlechten Kopplung des Druckfeldes in verschiedenen Gitterebenen und der daraus folgenden Instabilität der Berechnung. Dies konnte im vorliegenden Fall umgangen werden, indem eine besondere Lösungsvariante für das Druckfeld entwickelt wurde. Ebenso mußte für die Stabilisierung der speicherplatzsparenden Methode des Zellüberdeckungsgrades ein virtuelles Druckfeld innerhalb der Struktur berechnet werden. Ein so tiefer Eingriff in den Algorithmus ist bei kommerziellen Programmen nicht möglich.

- In kommerziellen Programmen werden meistens körperangepaßte Koordinaten verwendet. Dies erfordert gegenüber der vorliegenden Methode ein Vielfaches an Berechnungszellen am Umfang der Zylinder.

Die genannten Punkte führen in letzter Konsequenz zu der Einsicht, daß mit heutigen Computern die Lösung des vorliegenden Problems auf numerischem Wege ausschließlich über die Entwicklung eines eigenen Programms möglich 
war, bei dem an den entscheidenden Stellen der in kommerziellen Programmen gängige Berechnungsweg verändert werden konnte.

\subsection{Mögliche zukünftige Entwicklungen}

Für die Zukunft wird auf das Potential des vorgelegten Berechnungsprogramms zu folgenden Weiterentwicklungen hingewiesen:

- Relativ leicht kann eine Energiegleichung als neue Transportgleichung eingeführt werden. Als neue Feldgröße tritt die Temperatur hinzu.

- Mit der Einführung der Temperatur können dann auch Stoffgrößen als temperaturabhängige Größen eingeführt werden. Sowohl Strömungs- als auch Strukturmodell sind hierfür vorbereitet.

- Weiter könnte dann das Strömungsfeld innerhalb der Rohre freigegeben werden. Mit zusätzlichen Randbedingungen für die Durchströmung der Rohre und einer Betrachtung der instationären Temperaturleitung durch die Rohrwände wären damit Wärmeübergangsrechnungen ermöglicht. Corioliseffekte infolge der Durchströmung der verformten bewegten Rohre gingen dann auch in die mechanischen Bewegungsgleichungen mit ein.

- Ein wichtiger Strömungsfall in der Praxis sind Gasströmungen. Soweit es die im allgemeinen im Vergleich zur Schallgeschwindigkeit geringen Gasgeschwindigkeiten im Apparatebau zulassen, kann dieser Fall hinsichtlich der mechanischen Anregung von Rohrstrukturen ebenfalls inkompressibel berechnet werden. Hierfür müssen dann lediglich die Tabellierungen der Stoffwerte weit genug reichen.

- Über verallgemeinerte Routinen zur Berechnung des Überdeckungsgrades wären noch allgemeinere Geometriebeschreibungen möglich.

- Durch Einführung anderer Strukturmodelle wären dann auch allgemeinere fluid-struktur-gekoppelte Probleme berechenbar. Durch die Verwendung des Konzeptes des Zellüberdeckungsgrades bestehen hier grundsätzlich keine Einschränkungen hinsichtlich des Strömungsmodells. Zu denken wäre etwa an das Flattern von Ventiltellern, an Flatterschwingungen von Flügelprofilen oder an Rohrleitungsschwingungen.

Echt zweiphasige oder kompressible Strömungsberechnungen, etwa zur Untersuchung akustischer Resonanzfälle, würden einen deutlich höheren Weiterentwicklungsaufwand bedeuten als die in obiger Aufstellung angerissenen Möglichkeiten. Unbedingt erforderlich ist jedoch eine weitere Stabilisierung des vorhandenen Wissens hinsichtlich der numerischen Einstellparameter von fluid- 
struktur-gekoppelten Berechnungsverfahren. Hierbei spielt auch das verwendete Turbulenzmodell eine wichtige Rolle. Eine gesonderte Untersuchung sollte klären, welches Turbulenzmodell für Wärmetauscherdurchströmungen am günstigsten ist. Insbesondere sollten die derzeitigen Entwicklungen auf dem Gebiet der dynamischen Large-Eddy-Modelle sorgfältig verfolgt werden. Ein diesbezügliches Forschungsvorhaben wurde vom Verfasser noch auf den Weg gebracht [Fis99c].

Aufgrund der fortschreitenden Rechnertechnologie und der weiter fallenden Kosten für Speicherbausteine sind Speicherplatzprobleme zur Berechnung von Rohrbündeldurchströmungen inzwischen von eher untergeordneter Bedeutung. Die Berechnungen für die vorliegende Arbeit konnten hauptsächlich auf handelsüblichen PC-Rechnern durchgeführt werden. Die standardmäßige industrielle Anwendung des dargestellten numerischen Simulationsverfahrens stellt jedoch weiterhin große Anforderungen an die Rechengeschwindigkeit der verwendeten Computer. Für Großanwendungen sollte daher an eine Parallelisierung des Codes gedacht werden. 


\section{Literatur}

[Ada84] Adamson, J.: General Method for producing a boundary-fitted orthogonal curvilinear mesh. Applied Mathematic Modelling, Bd. 8, S. 231-237, 1984.

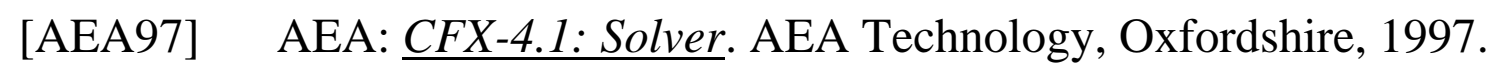

[Ant90] Antunes, J, Axisa, F., Beaufils, B., Guilbaud, D.: Coulomb Friction Modelling in Numerical Simulations of Vibration and Wear Work Rate of Multispan Tube Bundles. Journal of Fluids and Structures, Bd. 4, S. 287-304, 1990.

[Aut89] Autrata, M.: Vielstellige Arithmetik und Numerik in einer objektorientierten Softwareumgebung. Dissertation Universität Ulm, 1989.

[AuY98] Au-Yang, M.K.: Flow-Induced Wear in Steam Generator Tubes Prediction Versus Operational Experience. Transactions of the ASME, Journal of Pressure Vessel Technology, Bd. 120, S. 138143, Mai 1998.

[Axi88] Axisa, F., Antunes, J., Villard, B.: Overview of Numerical Methods for Predicting Flow-Induced Vibration. Transactions of the ASME, Journal of Pressure Vessel Technology, Bd. 110, S. 6-14, Februar 1988.

[Bar83] Bardina, J.: Improved Turbulence Models Based on Large Eddy Simulation of Homogeneous, Incompressible, Turbulent Flows. Dissertation Universität Stanford, 1983.

[Bar94] Barsamian, H.R., Hassan, Y.A.: Modified Subgrid Scale Model for Large Eddy Simulation of Tube Bundle Cross Flow. ASME PVP Bd. 273, Flow-Induced Vibration, S. 283-288, 1994.

[Bat86] Bathe, K.-J.: Finite-Elemente-Methoden. Springer-Verlag 1986.

[Bau85] Bauer, R.: Schwingungsschäden an Wärmetauschern. Arbeitssitzung Fachausschuß „Prozeß- und Anlagentechnik“ GVC-VDI- 
Gesellschaft Verfahrenstechnik und Chemieingenieurwesen, Breisach, 21./22.10.1985.

[Bau01] Bauer, L.: Entwicklung einer Bedienoberfläche für ein numerisches Tool zur Simulation von Rohrbündelschwingungen. Theoretische Semesterarbeit Lehrstuhl für Apparate- und Anlagenbau, TU München, 2001.

[Ber96] Bernert, K., Eppler, A.: Large-Eddy-Simulation - grundlagenorientierte und anwendungsbezogene Untersuchungen zu Modellierungs- und numerischen Komponenten. Forschungsbericht TU Chemnitz-Zwickau, 1996.

[Bir98] Birkner, M.: Weiterentwicklung eines dreidimensional rechnenden Fortranprogramms zur Berechnung der Strömungskräfte auf umströmte Wärmetauscherrohre. Theoretische Diplomarbeit Lehrstuhl für Apparate- und Anlagenbau, TU München, 1998.

[Bir00] Birtigh, A., Lauschke, G., Schierholz, W.F., Beck, D., Maul, C., Gilbert, N., Wagner, H.-G., Werninger, C.Y.: CFD in der chemischen Verfahrenstechnik aus industrieller Sicht. Chemie Ingenieur Technik, Bd. 72, Heft 3, S. 175-193, 2000.

[Bis93] Bischoff, M.: Simulation von Gasströmungen mit Temperatur- und Geschwindigkeitsschieflagen durch Rohrbündel. Dissertation TU Hamburg-Harburg, 1993.

[Ble79] Blevins, R.D.: Fretting Wear of Heat Exchanger Tubes. Part I: Experiments. Part II: Models. Transactions of the ASME, Journal of Engineering for Power, Bd. 101, S. 625-633, Oktober 1979.

[Ble85] Blevins, R.D.: Vibration-Induced Wear of Heat Exchanger Tubes. Transactions of the ASME, Journal of Engineering Materials and Technology, Bd. 107, S. 61-67, Januar 1985.

[Böh87] Böhm, J.: A Comparison of Different Contact Algorithms With Applications. Computers and Structures, Bd. 26, Nr. 1/2, S. 207221, 1987. 
[Bot00] Botros, K.K., Price, G., May, D., Holding, C.: A case study of fluid-elastic instability of a large heat exchanger in a petrochemical process plant. In: Ziada, S., Staubli, T. (Herausgeber): Flow Induced Vibration. Balkema Verlag, Rotterdam, S. 489-496, 2000.

[Bra98] Braun, W., Lang, A.: Einsatz numerischer Strömungsmechanik zur Auslegung und Optimierung von Rauchgasreinigungsanlagen. VGB Kraftwerkstechnik 6/98, S. 87-93, 1998.

[Bra85] Braza, M., Chassaing, P., Ha Minh, H.: A Numerical Study of the Dynamics of Different Scale Structures in the Near Wake of a Circular Cylinder in Laminar to Turbulent Transition. Proceedings $4^{\text {th }}$ International Conference on Numerical Methods in Laminar and Turbulent Flow, Swansea, S. 672-686, 1985.

[Bro87] Bronstein, I.N.: Semendjajew, K.A.: Taschenbuch der Mathematik. Verlag Harri Deutsch, Thun und Frankfurt/Main, 23. Auflage, 1987.

[Bur71] Burger, S.: Einfluß hydrodynamischer Krafteinwirkungen auf die Biegeschwingungen von Rührwellen. Chemie Ingenieur Technik Bd. 43, Nr. 6, S. 392-397, 1971.

[Can83] Cantwell, B., Coles, D.: An experimental study of entrainment and transport in the turbulent near wake of a circular cylinder. Journal of Fluid Mechanics, Bd. 136, S. 321-374, 1983.

[Cha87] Cha, J.H., Wambsganss, M.W., Jendrzejczyk, J.A.: Experimental Study on Impact/Fretting Wear in Heat Exchanger Tubes. Transactions of the ASME, Journal of Pressure Vessel Technology, Bd. 109, S. 265-274, August 1987.

[Cha89] Chang, Y., Beris, A.N., Michaelidis, E.E.: A Numerical Studiy of Heat and Momentum Transfer for Tube Bundles in Cross-Flow. International Journal for Numerical Methods in Fluids, Bd. 9, S. 1381-1394, 1989.

[Cha00] Chan, S.T., Lam, K., So, R.M.C., Leung, R.C.K.: Numerical study of flow around circular cylinder. In: Ziada, S., Staubli, T. (Hrsg.): 
Flow Induced Vibration. Balkema, Rotterdam, S. 249-255, 2000.

[Che76] Chen, S.S., Wambsganss, M.W., Jendrzejczyk, J.A.: Added Mass and Damping of a Vibrating Rod in Confined Viscous Fluids. Transactions of the ASME, Journal of Applied Mechanics, S. 325329, Juni 1976.

[Che85] Chen, S.S., Jendrzejczyk, J.A., Wambsganss, M.W: Dynamics of Tubes in Fluid With Tube-Baffle Interaction. Transactions of the ASME, Journal of Pressure Vessel Technology, Bd. 107, S. 7-17, Februar 1985.

[Che88] Chen, C.-J.: Finite Analytic Method. In: Minkowycz, W.J.: Handbook of Numerical Heat Transfer (Kap. 17). John Wiley \& Sons, 1988.

[Che95] Chen, C.-J., Bravo, R.H., Chen, H.-C., Xu, Z.: Accurate Discretisation of Incompressible Three-Dimensional Navier-Stokes Equations. Numerical Heat Transfer, Part B, Bd. 27, S. 371-392, 1995.

[Che73] Chen, Y.N.: 60 Jahre Forschung über die Kármánschen Wirbelstraßen - Ein Rückblick. Schweizerische Bauzeitung, 91. Jahrgang, Heft 44, S. 1079-1096, 1973.

[Che99] Cheng, M.: Vortex Shedding Lock-In Phenomenon of a Circular Cylinder Near a Plane Boundary. ASME PVP Bd. 396, Emerging Technologies in Fluids, Structures, and Fluid/Structure Interactions, Boston, MA, USA, 1.-5.8.1999, S. 51-57, 1999.

[Cho67a] Chorin, A.J.: A Numerical Method for Solving Incompressible Viscous Flow Problems. Journal of Computational Physics 2, S. 12-26, 1967.

[Cho67b] Chorin, A.J.: The Numerical Solution of the Navier Stokes Equations for an Incompressible Fluid. Bulletin of the American Mathematical Society, Nr. 73, S. 928-931, 1967.

[Cho95] Choi, S.K., Nam, H.Y., Cho, M.: Evaluation of a Higher-Order Bounded Convection Scheme: Three-Dimensional Numerical 
Experiments. Numerical Heat Transfer Part B, Bd. 28, S. 23-28, 1995.

[Cho98] Choi, S.K.: Persönliche Mitteilung. Daejon, Korea, 1998.

[Chu83] Chung, T.J.: Finite Elemente in der Strömungsmechanik. Carl Hanser Verlag München Wien, 1983.

[Con78] Connors, H.J.: Fluidelastic Vibration of Heat Exchanger Tube Arrays. Transactions of the ASME, Journal of Mechanical Design, Bd. 100, S. 347-353, 1978.

[Dal83] Dallmann, H., Elster, K.-H.: Partielle Differentialgleichungen. In: Einführung in die höhere Mathematik 3. Vieweg Verlag, Braunschweig, 1983

[Dei01] Deininger, J., Fischer, M.: Berechnung der Lateralsteifigkeiten von Rohren in Rohrbündelwärmetauschern. Interner Bericht Lehrstuhl für Apparate- und Anlagenbau, TU München, 2001.

[Dem88] Demirdzic, I., Peric, M.: Space Conservation Law in Finite Volume Calculations of Fluid Flow. International Journal for Numerical Methods in Fluids, Bd. 8, S. 1037-1050, 1988.

[Dem90] Demirdzic, I., Peric, M.: Finite Volume Method for Prediction of Fluid Flow in Arbitrarily Shaped Domains with Moving Boundaries. International Journal for Numerical Methods in Fluids, Bd. 10, S. 771-790, 1990.

[DIN80] DIN 28182: Rohrbündel-Wärmeaustauscher: Rohrteilungen und Rohrbefestigungen. Deutsches Institut für Normung e.V., Januar 1980.

[DIN91] DIN EN 10002: Zugversuch. Teil 1: Prüfverfahren. Deutsches Institut für Normung e.V., April 1991.

[Dra98] Dragon, D.: Konstruktion einer Befestigungsvorrichtung für PiezoBeschleunigungsaufnehmer in Wärmetauscherrohren. Konstruktive Semesterarbeit Lehrstuhl für Apparate- und Anlagenbau, TU 
München, 1998.

[Dra99] Dragon, D.: Numerische Parameterstudie der Spaltströmungen in Rohrbündelwärmetauschern. Theoretische Semesterarbeit Lehrstuhl für Apparate- und Anlagenbau, TU München, 1999.

[Dre56] Drescher, H.: Messung der auf querangeströmte Zylinder ausgeübten zeitlich veränderten Drücke. Zeitschrift für Flugwissenschaft, Bd. 4, Heft 1/ 2, S. 17-21, 1956.

[Dre59] Drescher, H.: Zur Mechanik der Reibung zwischen festen Körpern. VDI-Zeitschrift, Bd. 101, Nr. 17, S. 697-732, 1959.

[Eca96] Eça, L.: 2D Orthogonal Grid Generation with Boundary Point Distribution Control. Journal of Computational Physics, Vol. 125, pp. 440-453, 1996.

[Eng94] Engel, R. Analysis of fluid-structure interaction problems in nuclear engineering. International Journal of Computer Applications in Technology, Bd. 7, Nr. 3-6, S. 193-205, 1994.

[Fer99] Ferziger, J.H., Peric, M.: Computational Methods for Fluid Dynamics. Springer Verlag Berlin, Heidelberg, New York, 2. Auflage, 1999.

[Fis95a] Fischer, M.: Untersuchung verschiedener Diskretisierungsmöglichkeiten gekrümmter Oberflächen in zweidimensional rechnenden CFD-Verfahren. Theoretische Diplomarbeit Lehrstuhl für Apparate- und Anlagenbau, TU München, 1995.

[Fis95b] Fischer, M.: Konzeption eines genauen numerischen Verfahrens zur Berechnung turbulenter Rohrbündeldurchströmungen. Interner Bericht Lehrstuhl für Apparate- und Anlagenbau, TU München, 1995.

[Fis98a] Fischer, M., Strohmeier, K.: Schwingungssichere Auslegung querangeströmter Rohrbündelwärmetauscher mithilfe numerischer Simulation. Konferenzband VGB-Jahrestagung, Essen, 11. und 
12.2.1998.

[Fis98b] Fischer, M., Strohmeier, K.: Simulation of the Two-Phase Secondary Flow in an Acetylene Cooler. Chemical Engineering and Technology, Bd. 21, Nr. 10, S. 791-794, 1998.

[Fis98c] Fischer, M., Strohmeier, K.: Simulation der zweiphasigen Sekundärströmung in einem Acetylen-Kühler. Chemie Ingenieur Technik Bd. 70, Nr. 11, S. 1463-1466, 1998.

[Fis99a] Fischer, M., Dragon, D., Strohmeier, K.: Über analytische und numerische Berechnungen laminarer Strömungen innerhalb eines Sichelspalts zwischen einem bewegten Wärmetauscherrohr und einer Leitblechbohrung. Konferenzband 19. DECHEMAKonstruktions-Symposium, DECHEMA-Haus, Frankfurt/Main, S. 145-154, 4.5.1999.

[Fis99b] Fischer, M.: Haftverband durch hydraulisches Aufweiten. In: Strohmeier, K.: Übungen zur Vorlesung „Konstruktion im Anlagenbau für Chemieingenieure" (Aufgabe 3.2). Skriptum Lehrstuhl für Apparate- und Anlagenbau, TU München, 1999.

[Fis99c] Fischer, M., Strohmeier, K.: Richtlinien für die numerische Simulation von strömungsinduzierten Schwingungen in Rohrbündelwärmetauschern. Vortrag zum Anschlußantrag zu AiFForschungsvorhaben 11196 N/1, DECHEMA-Haus, Frankfurt/Main, DECHEMA-Ausschuß „Beanspruchungsgerechtes Konstruieren“, 2.11.1999.

[Fis00a] Fischer, M., Strohmeier, K.: Entwicklung eines Berechnungsverfahrens zur Simulation von strömungsinduzierten Schwingungen in Rohrbündelapparaten. Schlußbericht zu AiF-Forschungsvorhaben $11196 \mathrm{~N} / 1$, 31.5.2000.

[Fis00b] Fischer, M., Strohmeier, K.: The Use of Algebraic Computer Systems to Find Analytical Solutions in Continuum Mechanics and Applications to Fluid-Structure Interaction Problems. ASME PVP Bd. 414-2, Emerging Technologies in Fluids, Structures, and 
Fluid/Structure Interactions, Seattle, WA, USA, 23.-27.7.2000, S. $65-72,2000$.

[Fis01a] Fischer, M., Strohmeier, K.: Entwicklung eines Berechnungsverfahrens zur Simulation von strömungsinduzierten Schwingungen in Rohrbündelapparaten. Chemie Ingenieur Technik, Bd. 73, Nr. 4, S. 400-404, 2001

[Fis01b] Fischer, M., Strohmeier, K.: Korrosionsschaden an einem Rohrbündelwärmetauscher und Entwicklung von Abwehrmaßnahmen. Angenommen zur Veröffentlichung in: Technische Überwachung.

[Fis02] Fischer, M., Strohmeier, K.: Safety in Tube Bundle Heat Exchangers: Some Remarks on the Latest German Standard Concerning Flow-Induced Vibration. ASME PVP, Emerging Technologies in Fluids, Structures, and Fluid/Structure Interactions, Vancouver, BC, Kanada, 4.-8.8.2002.

[Fra91] Franke, R.: Numerische Berechnung der instationären Wirbelablösung hinter zylindrischen Körpern. Dissertation TH Karlsruhe, 1991.

[Fre97] Freitas, C.J., Runnels, S.R.: Simulation of Fluid-Structure Interaction using Patched-Overset Grids. ASME PVP Bd. 355, Advances in Analytical, Experimental and Computational Technologies in Fluids, Structures, Transients and Natural Hazards, S. 37-56, 1997.

[Fri94] Friedrich, R.: Grundlagen kompressibler Turbulenz. Mitschrift der Vorlesung, Lehrstuhl für Fluidmechanik, TU München, 1994.

[Fuj01] Fujita, K., Nakamura, T.: Physical Consideration in Practical Use of Numerical Simulation for a Vortex-Induced Vibration of Single Circular Cylinder. PVP Bd. 420-1, Flow-Induced Vibration, Atlanta, GA, USA, 22.-26.7.2001, S. 185-194, 2001.

[Gas88] Gaskell, P.H., Lau, A.K.C.: Curvature-Compensated Convective Transport: SMART, a New Boundedness-Preserving Transport Algorithm. International Journal for Numerical Methods in Fluids, 
Bd. 8, S. 617-641, 1988.

[Gel94] Gelbe, H.: Schwingungen und Geschwindigkeitsverteilungen in Rohrbündeln mit Umlenkungen. Schlußbericht zu AiF-Forschungsvorhaben 8580, 1994.

[Gel98] Gelbe, H., Schröder, K.: Bestimmung der fluidelastischen Instabilität in querangeströmten Rohrbündeln. Chemie Ingenieur Technik, Bd. 70, Nr. 1 und 2, S. 80-88, 1998.

[Ger91] Germano, M., Piomelli, U., Moin, P., Cabot, W.H.: A dynamic subgrid-scale eddy viscosity model. Physics of Fluids A, Bd. 3, Nr. 7, S. 1760-1765, 1991.

[Gor78] Gordon, D.: Numerical Calculations on Viscous Flow Fields through Cylinder Arrays. Computers and Fluids, Bd. 6, S. 1-13, 1978.

[Gri95] Griebel, M., Dornseifer, T., Neunhoeffer, T.: Numerische Simulation in der Strömungsmechanik. Vieweg Verlag, Braunschweig, 1995.

[Gui00] Guilmineau, E., Queutey, P.: A numerical simulation of the response of a vortex-excited cylinder. In: Ziada, S., Staubli, T. (Hrsg.): Flow Induced Vibration. Balkema, Rotterdam, S. 257-264, 2000 .

[Ham97] Hamid, R.-Q.: Simulation von stationären dreidimensionalen Strömungs- und Temperaturfeldern in einem Rohrbündelwärmeübertrager. Dissertation Universität der Bundeswehr Hamburg, 1997.

[Har65] Harlow, F.H., Welch, J.E.: Numerical Calculation of TimeDependent Viscous Incompressible Flow of Fluid with Free Surface. The Physics of Fluids, Bd. 8, Nr. 12, 1965.

[Has55] Hastings, C.: Approximations for Digital Computers. Princeton University Press, 1955. 
[Has90] Hassan, Y.A., Bagwell, T.G.: Large Eddy Simulation on Supercomputers. Texas A\&M University, Department of Nuclear Engineering, Schlußbericht Forschungsprojekt S410-12, 1990.

[Hau81] Haussling, H.J., Coleman, R.M.: A Method for Generation of Orthogonal and Nearly Orthogonal Boundary-Fitted Coordinate Systems. Journal of Computational Physics, Bd. 43, S. 373-381, 1981.

[Her82] Hertz, H.: Ueber die Berührung fester elastischer Körper. Journal für die reine und angewandte Mathematik, Bd. 92, Nr. 2, S. 156$171,1882$.

[Hö197] Hölzl, R.: Persönliche Mitteilung. Garching bei München, 1997.

[Hö198] Hölzl, R.: Beanspruchungssimulation an hydraulisch gefügten Rohr-Rohrplattenverbindungen. Dissertation Lehrstuhl für Apparate- und Anlagenbau, TU München, 1998.

[Ich94] Ichioka, T., Kawata, Y., Izumi, H., Nakamura, T., Fujita, K.: TwoDimensional Flow Analysis of Fluid-Structure Interaction Around a Cylinder and a Row of Cylinders. ASME PVP Bd. 273, FlowInduced Vibration, S. 33-41, 1994.

[Ich95] Ichioka, T., Kawata, Y., Izumi, H., Nakamura, T., Takamatsu, H.: Two Dimensional Flow Analysis of Fluid-Structure Interaction Around a Tube Row and Tube Arrays in Cross-Flow. $3^{\text {rd }}$ International Conference on Nuclear Engineering (ICONE-3), Kyoto, Proceedings S125-2, S. 603-608, 1995.

[Irm94] Irmisch, S., Schwolow, R.: Erzeugung unstrukturierter Dreiecknetze mittels Delaunay-Triangulierung. Zeitschrift für Flugwissenschaften und Weltraumforschung, Bd. 18, S. 361-368, 1994.

[Jah95] Jahr, M.: Einflüsse von Strukturparametern und Strömungsverteilung auf das Schwingverhalten mit Luft angeströmter Rohrbündel. Dissertation TU Berlin, 1995.

[Jen82] Jendrzejczyk, J.A., Chen, S.S.: Fluid Forces Acting On Circular 
Cylinders in Liquid Cross Flow. Flow-Induced Vibration of Circular Cylinders, ASME-PVP Bd. 63, S. 31-44, 1982.

[Joh97] Johansson, L.: Beam Motion With Unilateral Contact Constraints and Wear of Contact Sites. Transactions of the ASME, Journal of Pressure Vessel Technology, Bd. 119, S. 105-110, Februar 1997.

[Kaa00] Kaam, T.P.: Konstruktive Auslegung von Rohrbündelkonstruktionen durch numerische Bestimmung der instationär wirkenden Strömungskräfte. Konstruktive Semesterarbeit Lehrstuhl für Apparate- und Anlagenbau, TU München, 2000.

[Käm88] Kämmel, G., Franeck, H., Recke, H.-G.: Einführung in die Methode der finiten Elemente. Carl Hanser Verlag, München/Wien, 1988.

[Kaß80] Kaßner, W., Mayfarth, H., Pöschmann, H., Schulz, W.-D.: Korrosionsschutz von Aluminium durch anodische und chemische Oxidschichten, Anstriche, Emails und metallische Schichten. 592. Mitteilung aus der Zentralstelle für Korrosionsschutz, 33/34, Dresden, 1980 .

[Kas94] Kassera, V., Strohmeier, K.: Schwingungssichere Auslegung von Rohrbündeln in Wärmetauschern. Abschlußbericht zum Forschungsvorhaben AiF 8575, 1994.

[Kas95] Kassera, V., Kacem-Hamouda, L., Strohmeier, K.: Numerical Simulation of Flow-Induced Vibrations of a Tube Bundle in Uniform Cross-Flow. ASME PVP, Bd. 298, Flow-Induced Vibration, S. 37-43, 1995.

[Kas97a] Kassera, V.: Entwicklung eines numerischen Berechnungsverfahrens für Schwingungen querangeströmter Rohrbündel. Dissertation Lehrstuhl für Apparate- und Anlagenbau, TU München, 1997.

[Kas97b] Kassera, V., Strohmeier, K.: Simulation of Tube Bundle Vibrations Induced by Cross-Flow. Journal of Fluids and Structures, Bd. 11, S. 909-928, 1997. 
[Kas98] Kassera, V.: Persönliche Mitteilung. Garching bei München, 1998.

[Kaw83] Kawahara, M., Hirano, H.: A Finite Element Method for High Reynolds Number Viscous Fluid Flow Using Two Step Explicit Scheme. International Journal for Numerical Methods in Fluids, Bd. 3, S. 137-163, 1983.

[Kaw84] Kawamura, T., Kuwahara, K.: Computation of High Reynolds Number Flow Around a Circular Cylinder With Surface Roughness. AIAA-84-0340, 22 ${ }^{\text {nd }}$ Aerospace Sciences Meeting, Reno, USA, 1984.

[Ker84] Kern, J.: Wirtschaftliche Gesichtspunkte bei der Auslegung von Wärmetauschern. Chemie Ingenieur Technik, Bd. 56, Nr. 6, S. 432-440, 1984.

[Kle00] Klein, A.: Wärmeübertrager. ACHEMA Berichte, Chemie Ingenieur Technik, Band 72, Nr. 12, S. 1495-1499, 2000.

[Kli89] Klingbeil, E.: Tensorrechnung für Ingenieure. BI Hochschultaschenbuch Bd. 197, Wissenschaftsverlag Mannheim/Wien/Zürich, 2. Auflage, 1989.

[Knu79] Knudson, W.C., Surya Kumar, G.V.: Explicit Time Integration Operators for Nonlinear Structural Dynamics. Mitteilungen 51/1979 des Sonderforschungsbereichs 64, Universität Stuttgart 1979.

[Koh93] Kohlpaintner, W.: Berechnung von hydraulisch gefügten RohrRohrplattenverbindungen. Dissertation Lehrstuhl für Apparateund Anlagenbau, TU München, 1993.

[KoP84] Ko, P.L., Basista, H.: Correlation of Support Impact Force and Fretting-Wear for a Heat Exchanger Tube. Transactions of the ASME, Journal of Pressure Vessel Technology, Nr. 106, S. 69-77, Februar 1984.

[Kot84] Kotthoff, K., Haunhorst, H., Schleifer, F., Trambauer, K.: Erkenntnisse aus dem Ablauf ausländischer Vorkommnisse mit 
Dampferzeuger-Heizrohrbruch. Technische Mitteilungen, Bd. 77, Nr. 1, Januar 1984.

[Kre00] Kretschmar, M.: Numerische Modellierung des Glasmodells eines Rohrbündelwärmetauschers. Konstruktive Semesterarbeit Lehrstuhl für Apparate- und Anlagenbau, TU München, 2000.

[Kri76] Krips, H., Podhorsky, M.: Hydraulisches Aufweiten - ein neues Verfahren zur Befestigung von Rohren. VGB Kraftwerkstechnik, Nr. 56, Heft 7, S. 456-464, 1976.

[Kul89] Kull, H., Studer, H.U.: Strömungsinduzierte Schwingungen in Glattrohrwärmetauschern. Technische Rundschau Nr. 39, S. 1-18, 1989.

[Lad98] Lade, O., Krawietz, A.: Computeralgebrasysteme für die symbolische Bearbeitung ingenieurtechnischer Fragestellungen. Chemie Ingenieur Technik, Bd. 70, S. 1284-1287, 1998.

[Leo79] Leonard, B.P.: A Stable and Accurate Convective Modelling Procedure Based on Quadratic Upstream Interpolation. Computer Methods in Applied Mechanics and Engineering, Bd. 19, S. 59-98, 1979.

[Leo95] Leonard, B.P., Drummond, J.E.: Why You Should Not Use „Hybrid“, „Power-Law“ or Related Exponential Scheme For Convective Modelling - There are Much Better Alternatives. International Journal for Numerical Methods in Fluids, Bd. 20, S. 421-442, 1995.

[Ley93] Leyh, T.: Strömungsinduzierte Rohrbündelschwingungen in einem gasdurchströmten realen Wärmetauscher. Dissertation TU Berlin, 1993.

[Lie66] Lienhard, J.H.: Synopsis of Lift, Drag and Vortex Frequency Data for Rigid Circular Cylinders. Washington State University, College of Engineering Research Division, Bulletin 300, 1966.

[Li192] Lilly, D.K.: A proposed modification of the Germano subgrid- 
scale closure method. Physics of Fluids A, Bd. 4, Nr. 3, S. 633635, 1992.

[Liu00] Liu, Y., Chan, S.T., So, R.M.C., Lam, K.: Flow-induced vibration of an elastic cylinder at sub-critical Reynolds numbers. In: Ziada, S., Staubli, T. (Hrsg.): Flow Induced Vibration. Balkema, Rotterdam, S. 241-247, 2000.

[Luk79] Lukasiewicz, S.: Local loads in plates and shells. Sijthoff \& Noordhoff International Publishers B.V., Alphen aan den Rijn, Niederlande, und PWN-Polish Scientific Publishers, Warschau, Polen, 1979.

[MAR99] MARC: Dynamic Analysis With MARC. Unterlagen zur technischen Schulung MTU:99-300-1, München, 25.-26.2.1999.

[Mic95] Microsoft: Fortran PowerStation Version 4.0 Reference. Microsoft Corporation, 1995.

[Miy92] Miyata, H., Yamada, Y.: A Finite Difference Method for 3D Flows About Bodies of Complex Geometry in Rectangular Coordinate Systems. International Journal for Numerical Methods in Fluids, Bd. 14, S. 1261-1287, 1992.

[Mob80] Mobley, C.D., Stewart, R.J.: On the Numerical Generation of Boundary-Fitted Orthogonal Curvilinear Coordinate Systems. Journal of Computational Physics, Bd. 34, S. 124-135, 1980.

[Mul80] Mulcahy, T.M.: Fluid Forces on Rods Vibration in Finite Length Annular Regions. Transactions of the ASME, Journal of Applied Mechanics, Bd. 47, S. 234-240, Juni 1980.

[Mur99] Murakami, S., Iizuka, S.: CFD Analysis of Turbulent Flow Past Square Cylinder Using Dynamic LES. Journal of Fluids and Structures, Bd. 13, S. 1097-1112, 1999.

[Nak01] Nakamura, A., Okajima, A.: A Numerical Simulation and Vortex Structures of In-Line Oscillation of an Elastically Supported Circular Cylinder. PVP Bd. 420-1, Flow-Induced Vibration, 
Atlanta, GA, USA, 22.-26.7.2001, S. 177-184, 2001.

[Nat92] Natke, H.G.: Einführung in Theorie und Praxis der Zeitreihenund Modalanalyse. Vieweg-Verlag, Braunschweig/Wiesbaden, 3. Auflage, 1992.

[New59] Newmark, N.M.: A Method of Computation for Structural Dynamics. ASCE, Journal of the Engineering Mechanics Division, Bd. 85, S. 67-94, 1959.

[Nie81] Niemann, G.: Maschinenelemente. Band I: Konstruktion und Berechnung von Verbindungen, Lagern, Wellen. Springer Verlag Berlin/Heidelberg/New York, 1981.

[Nol92] Noll, B.: Evaluation of a Bounded High-Resolution Scheme for Combustor Flow Computations. AIAA Journal, S. 64-69, Januar 1992.

[No193] Noll, B.: Numerische Strömungsmechanik. Springer Verlag Berlin, Heidelberg, New York, 1993.

[Ode83] Oden, J.T., Pires, E.B.: Nonlocal and Nonlinear Friction Laws and Variational Principles for Contact Problems in Elasticity. Journal of Applied Mechanics, Bd. 50, S. 67-76, 1983.

[Oer95] Oertel, H., Laurien, E.: Numerische Strömungsmechanik. Springer Verlag, Berlin, Heidelberg, New York, 1995.

[Ols94] Olsson, M., Fuchs, L.: Significant Terms in Dynamic SGSModeling. In: Direct and Large-Eddy Simulation I, Kluwer Academic Publishers, S. 73-83, 1994.

[Paï80] Païdoussis, M.P.: Flow-Induced Vibrations in Nuclear Reactors, Practical Experience and State of Knowledge. In: Naudascher, E., Rockwell, D.: Practical Experience with Flow-Induced Vibrations. Springer Verlag, Berlin/Heidelberg/New York, 1980.

[Pat80] Patankar, S.V.: Numerical Heat Transfer and Fluid Flow. Verlag McGraw-Hill, 1980. 
[Pet91] Pettigrew, M.J., Taylor, C.E.: Fluidelastic Instability of Heat Exchanger Tube Bundles: Review and Design Recommendations. Transactions of the ASME, Journal of Pressure Vessel Technology, Bd. 113, S. 242-256, 1991.

[Pfe92] Pfeiffer, F.: Einführung in die Dynamik. Teubner Studienbücher, Stuttgart, 1992.

[Pio91] Piomelli, U., Cabot, W.H., Moin, P., Lee, S.: Subgrid-scale backscatter in turbulent and transitionsal flows. Physics of Fluids A, Bd. 3, Nr. 7, S. 1766-1770, Juli 1991.

[Pre86] Press, W.H., Flannery, B.P., Teukolsky, S.A., Vetterling, W.T.: Numerical Recipes. Cambridge University Press, Cambridge, 1986.

[Pri95] Price, S.J.: A Review of Theoretical Models for Fluidelastic Instability of Cylinder Arrays in Cross-Flow. Journal of Fluids and Structures, Bd. 9 , S. 463-518, 1995.

[Pri98] Prithiviraj, M., Andrews, M.J.: Three Dimensional Numerical Simulation of Shell-And-Tube Heat Exchangers. Part I: Foundation and Fluid Mechanics. Numerical Heat Transfer Part A, Bd. 33, S. 799-816, 1998.

[Pri01] Price, S.J.: An Investigation on the Use of Connors' Equation to Predict Fluidelastic Instability in Cylinder Arrays. PVP Bd. 420-1, Flow-Induced Vibration, Atlanta, GA, USA, 22.-26.7.2001, S. 117-124, 2001.

[Rog76] Roger, R.J., Pick, R.J.: On the Dynamic Spatial Response of a Heat-Exchanger Tube With Intermittent Baffle Contacts. Nuclear Engineering and Design, Bd. 36, S. 81-90, 1976.

[Rom98] Romberg, O.: Zum Turbulenzeinfluß auf das Schwingungsverhalten querangeströmter Rohrbündel. Dissertation Universität Hannover, 1998.

[RRZ93] RRZN: Fortran 90 - Ein Nachschlagewerk. Regionales Rechen- 
zentrum für Niedersachsen, Universität Hannover, 2. Auflage, 1993.

[Sad95] Sadaoka, N., Umegaki, K.: Analysis of Vortex-Induced Vibration in an Array of Tubes. ASME PVP, Bd. 298, Flow Induced Vibration, S. 29-36, 1995.

[Sat94] Sato, T., Imayama, Z.: Numerical Analysis of Acoustic Excitation Forces of a Tube Bundle in Cross-Flow. ASME PVP Volume 273, Symposium on Flow Induced Vibration, Minneapolis, S. 185-190, 1994.

[Saw97] Sawtschenko, T.: Numerische und experimentelle Untersuchungen zum Einfluß einer ungleichmäßigen Geschwindigkeitsverteilung auf Wärmeübergang, Dissipation und Fouling in den Rohrbündeln von Wärmetauschern. Dissertation Universität Kaiserslautern, 1997.

[Schö90] Schönung, B.E.: Numerische Strömungsmechanik. Springer Verlag, Berlin/Heidelberg/New York, 1990.

[Schr99] Schröder, K., Gelbe, H.: Simulation of Flow-Induced Vibration in Tube Bundles. ASME PVP Bd. 399, Flow-Induced Vibration, Boston, MA, USA, 1.-5.8.1999, S. 9-16, 1999.

[Sin89] Singh, P., Caussignac, P., Fortes, A., Joseph, D.D., Lundgren, T.: Stability of Periodic Arrays of Cylinders Across the Stream by Direct Simulation. Journal of Fluid Mechanics, Bd. 205, S. 553$571,1989$.

[Sma63] Smagorinsky, J.: General Circulation Experiments With the Primitive Equations. I: The Basic Experiment. Monthly Weather Review, Bd. 91, Nr. 3, S. 99-108, März 1963.

[Smi70] Smith, G.D.: Numerische Lösung von partiellen Differentialgleichungen. Vieweg Verlag, Braunschweig, 1970

[Son90] Song, C.C., Yuan, M.: Simulation of Vortex-Shedding Flow about a Circular Cylinder at High Reynolds Numbers. Journal of Fluids 
Engineering, Bd. 112, S. 155-163, 1990.

[Sto00] Stolz, J.: Strömungsinduzierte Schwingungen in Rohrbündelwärmetauschern unter dem Einfluß von Reibstößen in den Leitblechen. Experimentelle Semesterarbeit Lehrstuhl für Apparate- und Anlagenbau, TU München, 2000.

[Suh89] Suh, S.-H.: Die Finite Analytische Methode zur Simulation von Strömungsproblemen. Dissertation Universität Stuttgart, VDI Verlag, Düsseldorf, 1989.

[Sza56] Szabó, I.: Höhere Technische Mechanik. Springer Verlag Berlin/ Göttingen/Heidelberg, 1956.

[Tam88] Tamura, T., Tsuboi, K., Kuwahara, K.: Numerical Simulation of Unsteady Flow Patterns around a Vibration Cylinder. AIAA-880128, 26 ${ }^{\text {th }}$ Aerospace Sciences Meeting, Reno, 1988.

[TEM82] TEMA-Katalog: Standards of Tubular Exchange Manufacturers Association. Section 12, S. 224-230, 1982.

[The86] Theodossiou, V.M., Sousa, A.C.M.: An Efficient Algorithm for Solving the Incompressible Fluid Flow Equations. International Journal of Numerical Methods in Fluids, Bd. 6, S. 557-572,1986.

[Tro86] Troidl, H.: Strömungsinduzierte Schwingungen querangeströmter Rohrbündel bei versetzter und fluchtender Anordnung. Dissertation Lehrstuhl für Apparate- und Anlagenbau, TU München, 1986.

[Tru68] Truckenbrodt, E.: Strömungsmechanik. Springer-Verlag, Berlin/ Heidelberg/New York, 1968.

[Urb94] Urbas, L., Leyh, T., Jahr, M., Gelbe, H.: Berechnung der dreidimensionalen Geschwindigkeitsverteilung in Rohrbündelwärmeübertragern und Simulation der Schwingungsanregung. Chemie Ingenieur Technik, Bd. 7, S. 938-940, 1994.

[VDI97] Gelbe, H., Schröder, K., Ziada, S.: Schwingungen in Wärmeübertrager-Rohrbündeln. In: VDI-Wärmeatlas (Kapitel Oc), Springer- 
Verlag, Berlin/Heidelberg/New York, 8. Auflage, 1997.

[Wea82] Weaver, D.S., Koroyannakis, D.: The Cross-Flow Response of a Tube Array in Air. ASME Journal of Pressure Vessel Technology 104, S. 139-146, 1982.

[Wea83] Weaver, D.S., Koroyannakis, D.: Flow-Induced Vibrations of Heat Exchanger U-Tubes: A Simulation to Study the Effects of Asymmetric Stiffness. Journal of Vibration, Acoustic, Stress and Reliability in Design 105, S. 67-75, 1983.

[Wea00] Weaver, D.S., Ziada, S., Au-Yang, M.K., Chen, S.S., Païdoussis, Pettigrew, M.J.: Flow-Induced Vibrations in Power and Process Plant Components - Progress and Prospects. Journal of Pressure Vessel Technology, Bd. 122, August 2000, S. 339-348.

[Wen93] Wengle, H.: Numerische Berechnung turbulenter Strömungen. Unterlagen zur Vorlesung, Lehrstuhl für Strömungsmechanik, TU München, 1993.

[Wil94] Wilcox, D.C.: Turbulence Modeling for CFD. DCW Industries Inc., La Cañada, CA, USA, 1994.

[Wil00] Willden, R.J.H., Graham, J.M.R.: Vortex induced vibration of deep water risers. In: Ziada, S., Staubli, T. (Hrsg.): Flow Induced Vibration. Balkema, Rotterdam, S. 29-36, 2000.

[Wo197] Wolfram, S.: Das Mathematica Buch. Verlag Addison-WesleyLongman, 3. Auflage, 1997.

[Zen87] Zeng, X., Li, W.: The Stability and Convergence of FAM for Unsteady Two-Dimensional Convective Transport Equation. Turbulence Measurements and Flow Modeling, 1987.

[Zha92] Zhang, S.-X.: Accurate Computation and Interpolation Techniques of Finite Analytic Coefficients. Applied Mathematics and Mechanics, Bd. 13, Nr. 5, S. 437-444, 1992.

[Zho96] Zhou, T., Rogers, R.J.: Simulation of Two-Dimensional Squeeze 
Film and Solid Contact Forces Acting on a Heat Exchanger Tube. ASME PVP, Bd. 328, Flow-Induced Vibration, S. 257-270, 1996.

[Zie84] Zienkiewicz, O.C.: Methode der Finiten Elemente. Carl Hanser Verlag München Wien, 2. Auflage, 1984. 


\section{Anhang: Farbabbildungen}

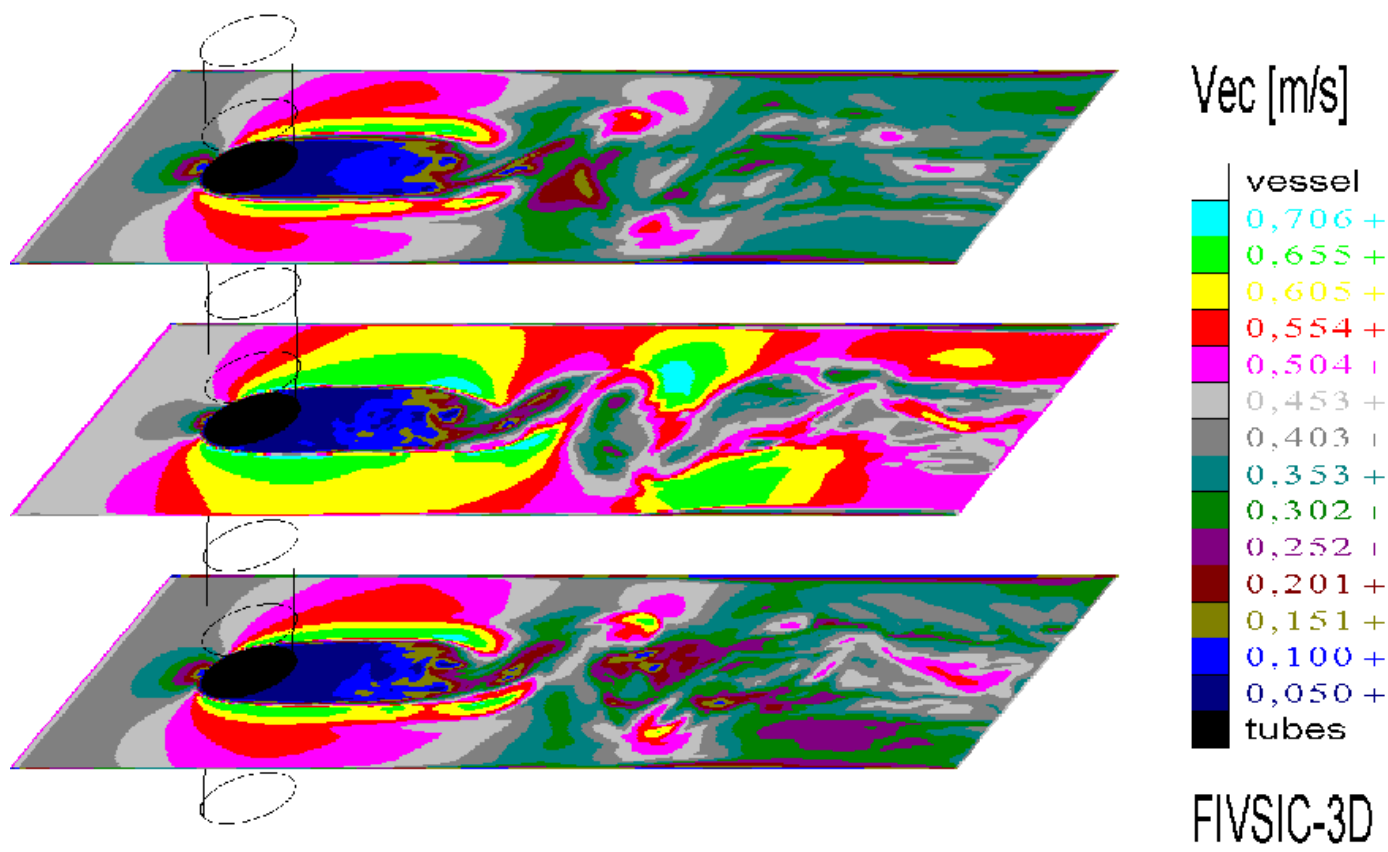

Abbildung 7-1: Momentaufnahme der Geschwindigkeitsverteilung bei homogen angeströmtem elastischen Einzelzylinder in einem Kanal.
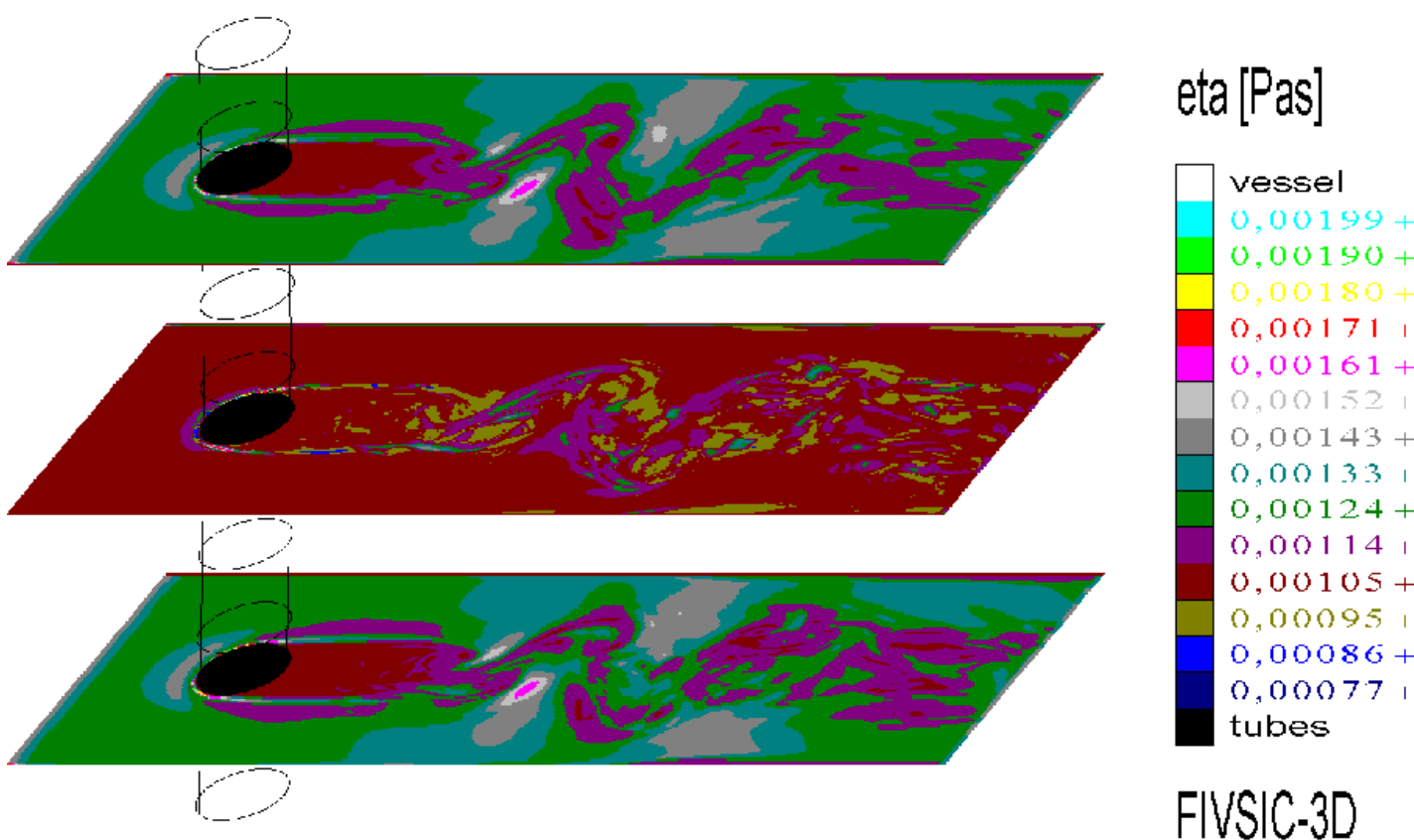

Abbildung 7-2: Momentaufnahme der Verteilung der Gesamtviskosität bei homogen angeströmtem elastischen Einzelzylinder in einem Kanal. 


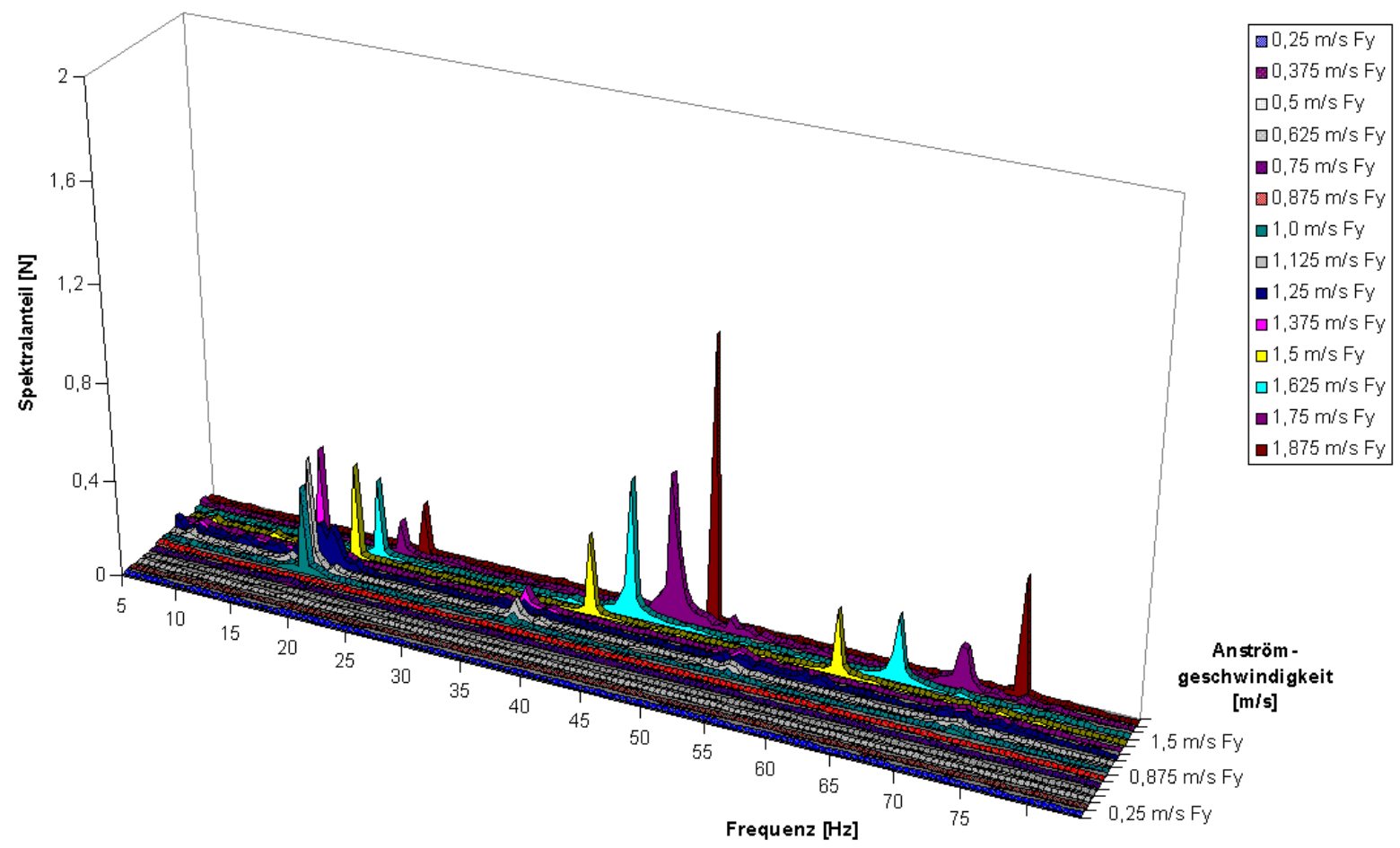

Abbildung 7-3: Fouriertransformation der Strömungskräfte auf einen schwingenden Einzelzylinder in Anströmrichtung.

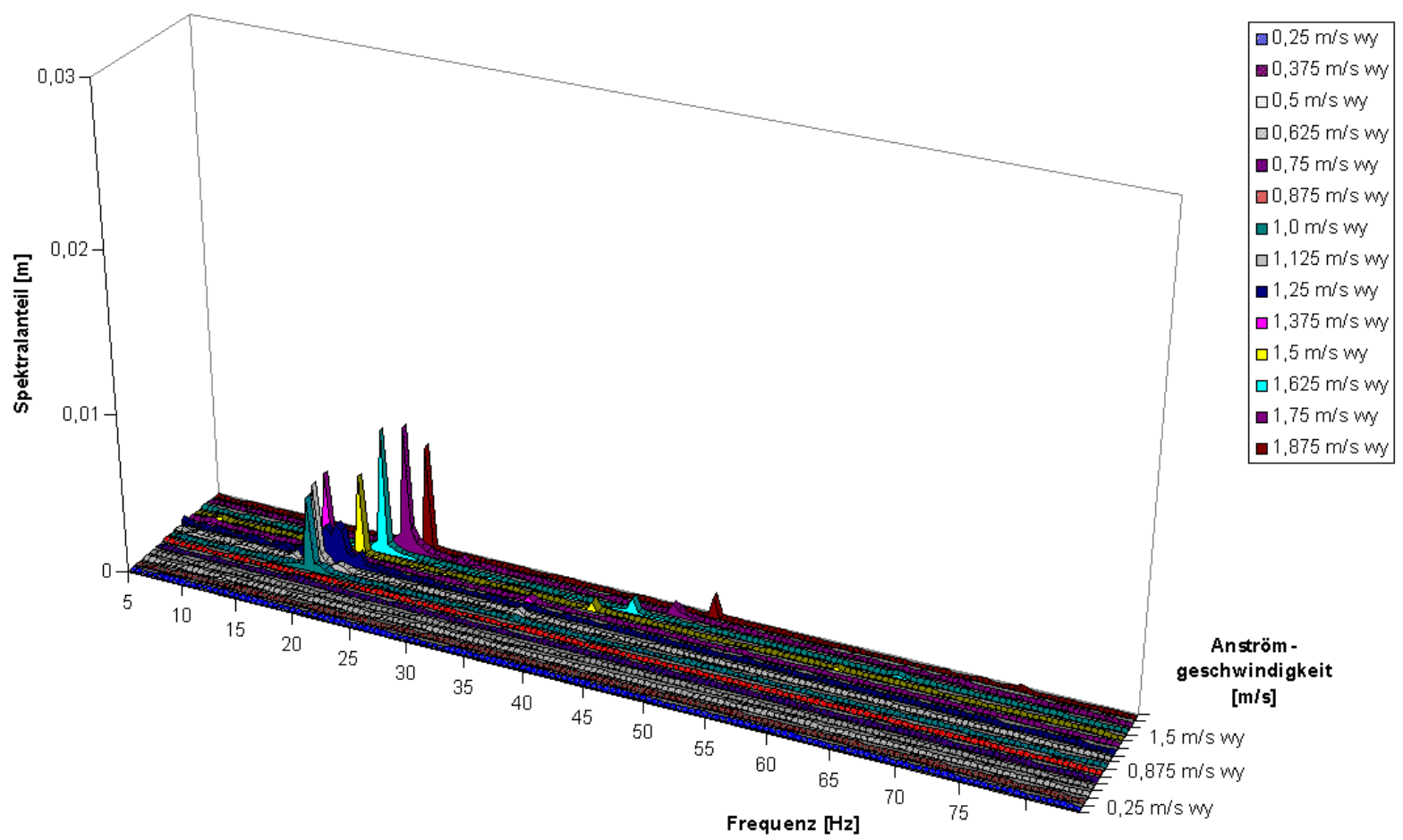

Abbildung 7-4: Fouriertransformation der Auslenkung eines schwingenden Einzelzylinder in Anströmrichtung. 


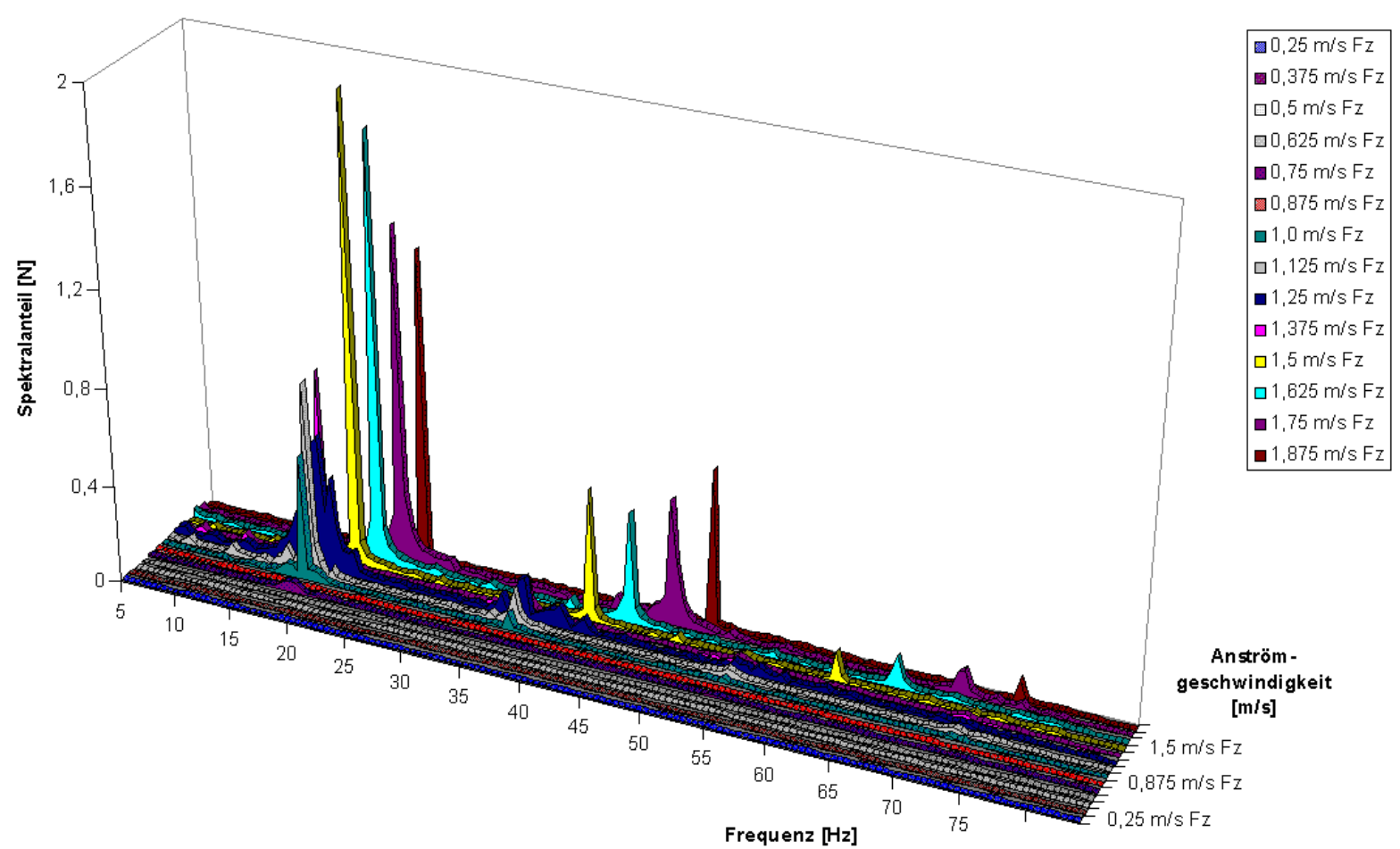

Abbildung 7-5: Fouriertransformation der Strömungskräfte auf einen schwingenden Einzelzylinder quer zur Anströmrichtung.

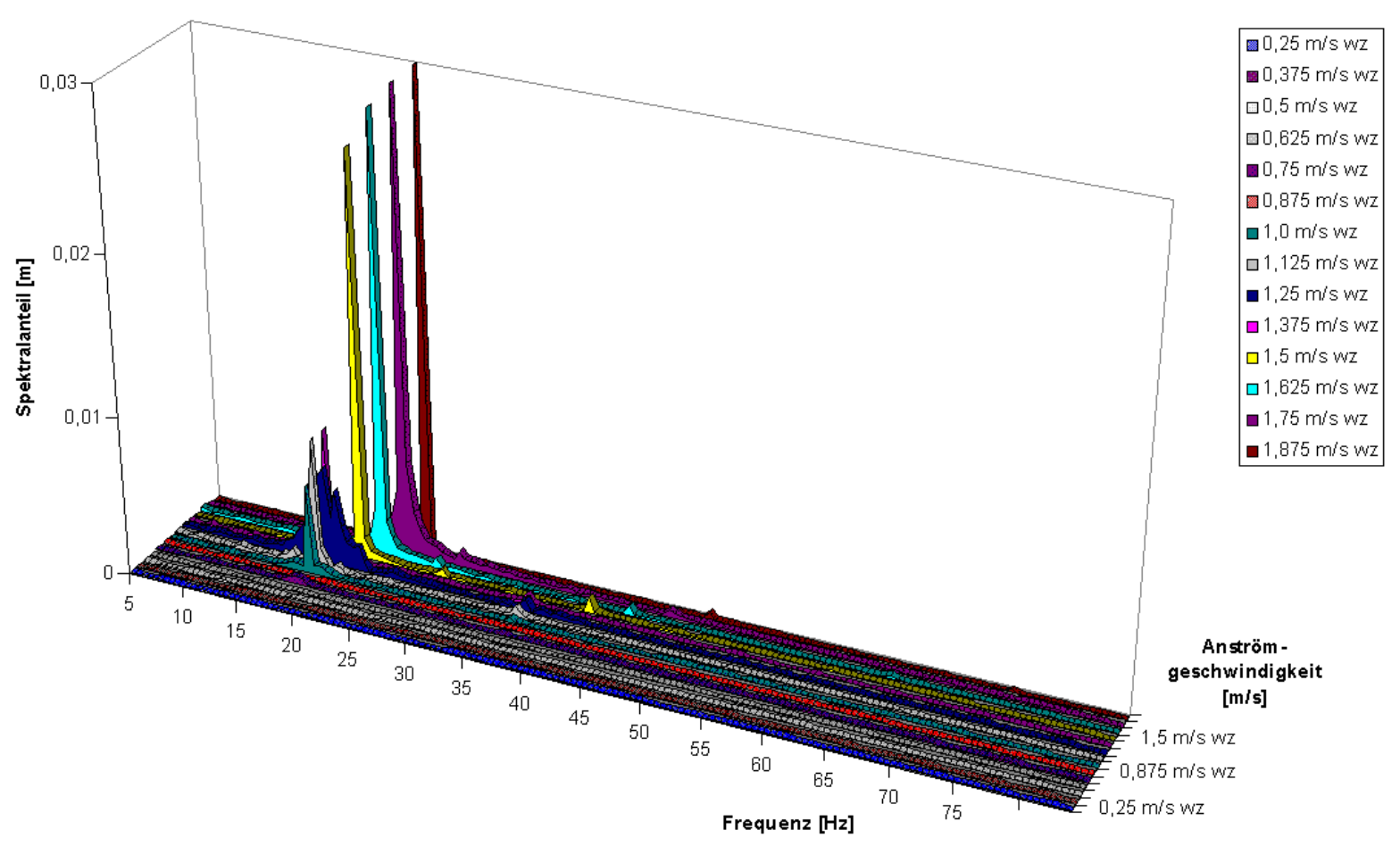

Abbildung 7-6: Fouriertransformation der Auslenkung eines schwingenden Einzelzylinder quer zur Anströmrichtung. 


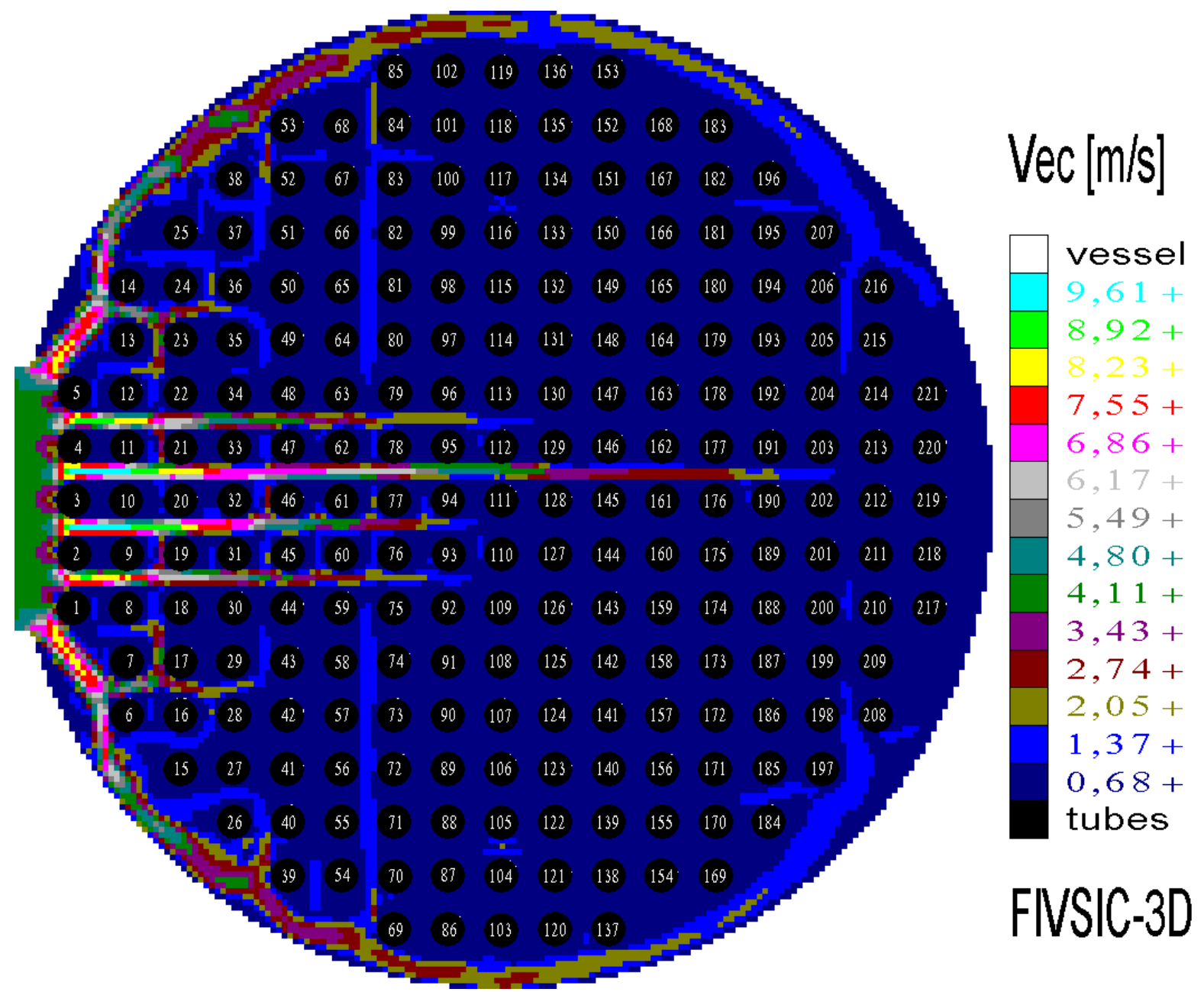

Abbildung 7-7: Geschwindigkeitsverteilung in der Eintrittsebene von BB1-L170 bei einem Volumenstrom von 100 m³/h.
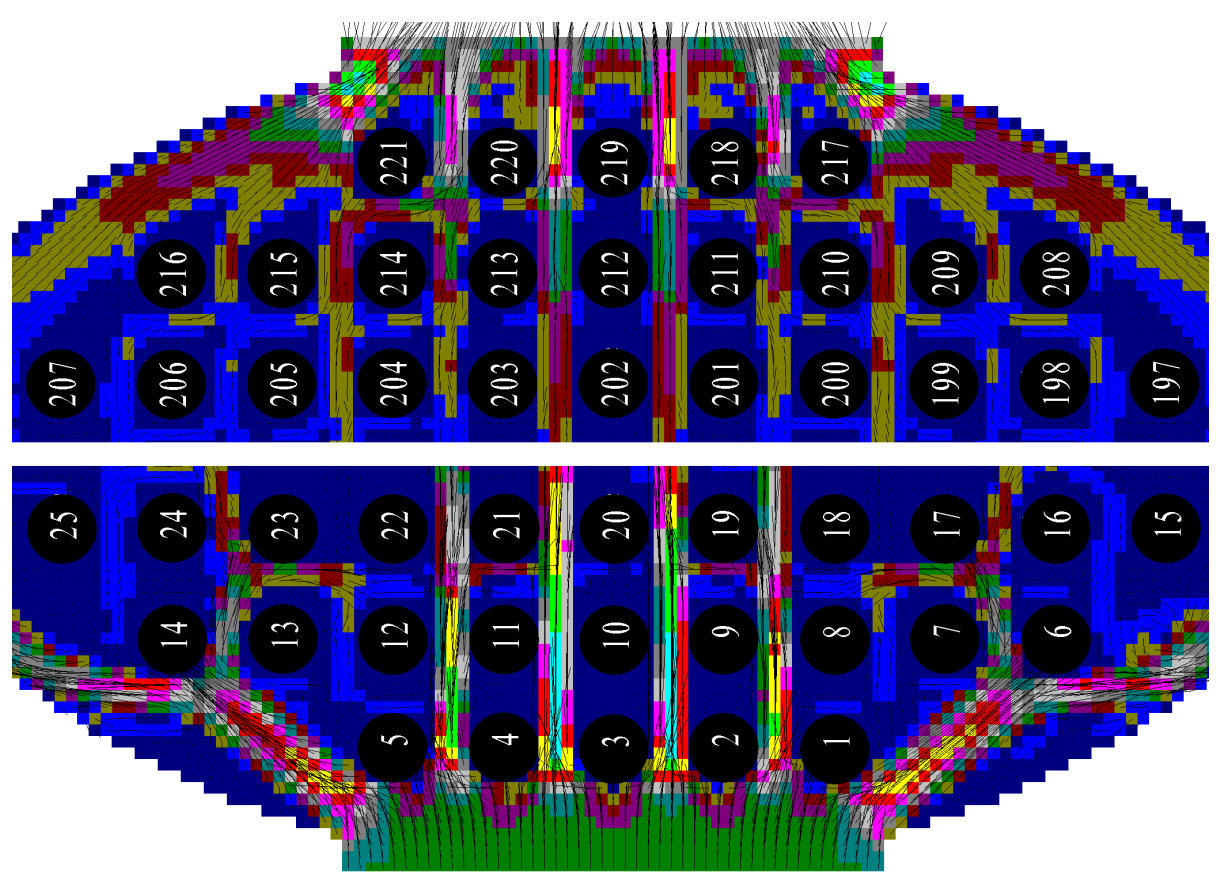

Abbildung 7-8: Detailansicht Eintritts- und Austrittsebene von Abbildung 7-7. 


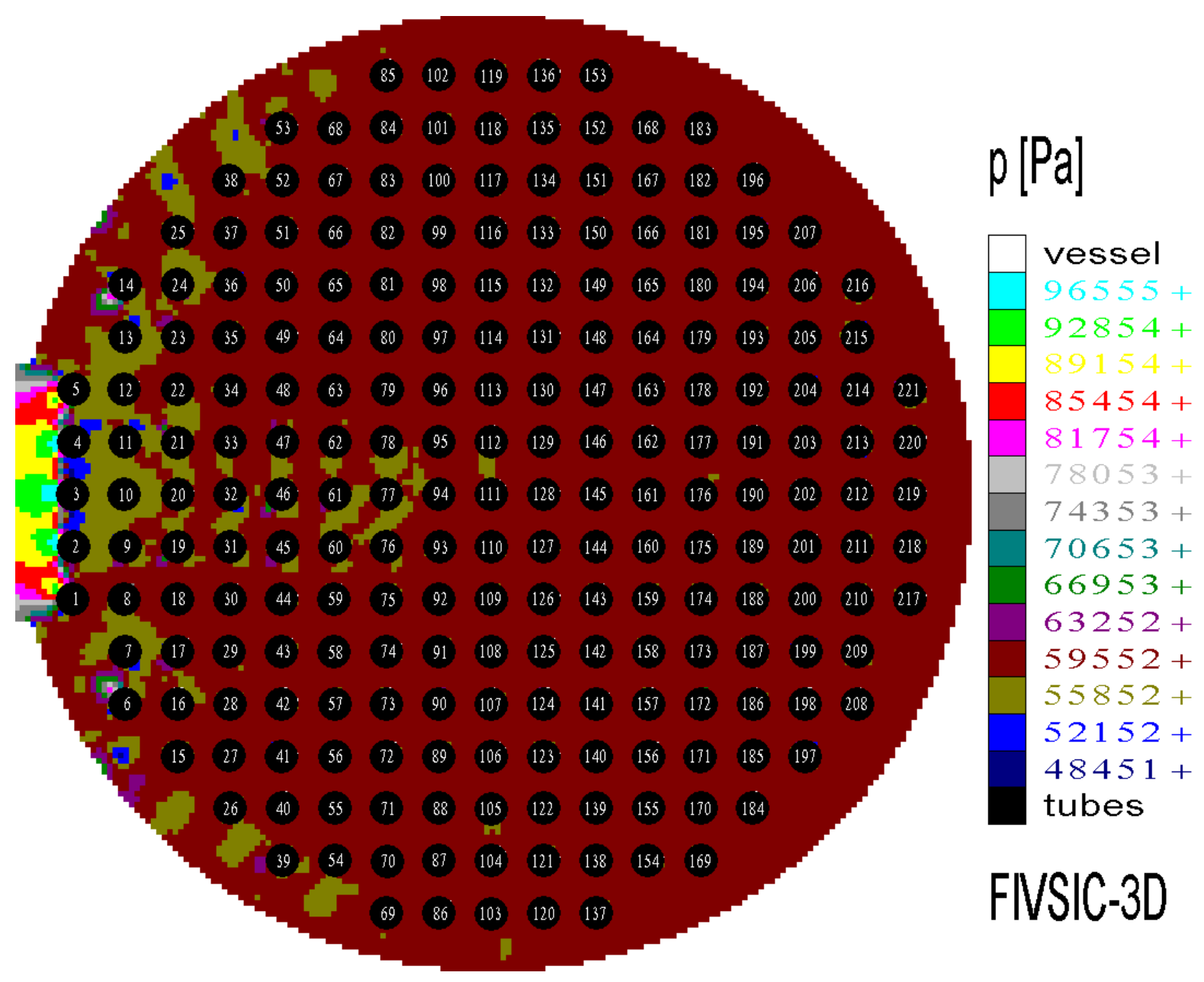

Abbildung 7-9: Druckverteilung in der Eintrittsebene von BB1-L170 bei einem Volumenstrom von $100 \mathrm{~m}^{3} / \mathrm{h}$.
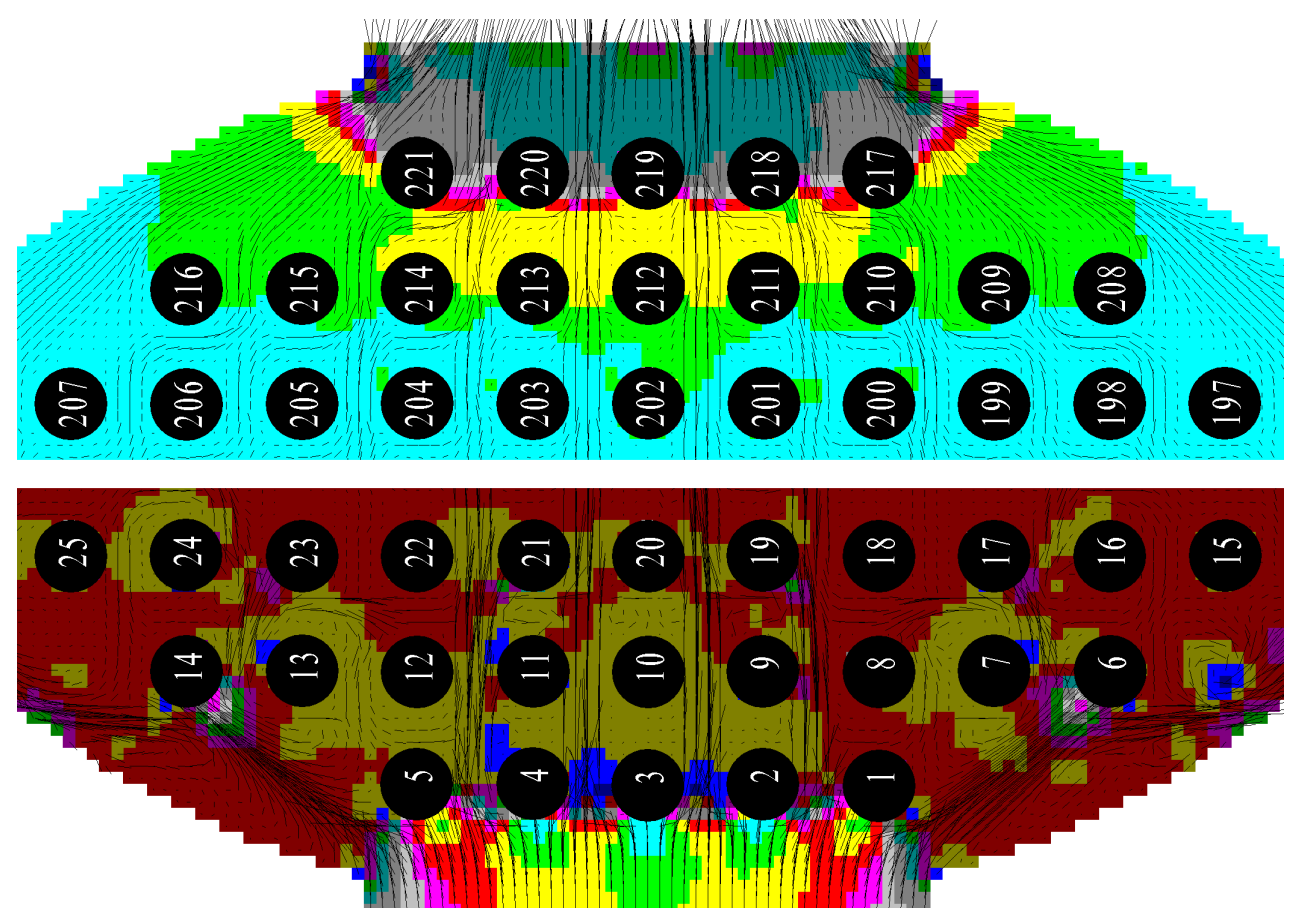

Abbildung 7-10: Detailansicht Eintritts- und Austrittsebene von Abbildung 7-9. 

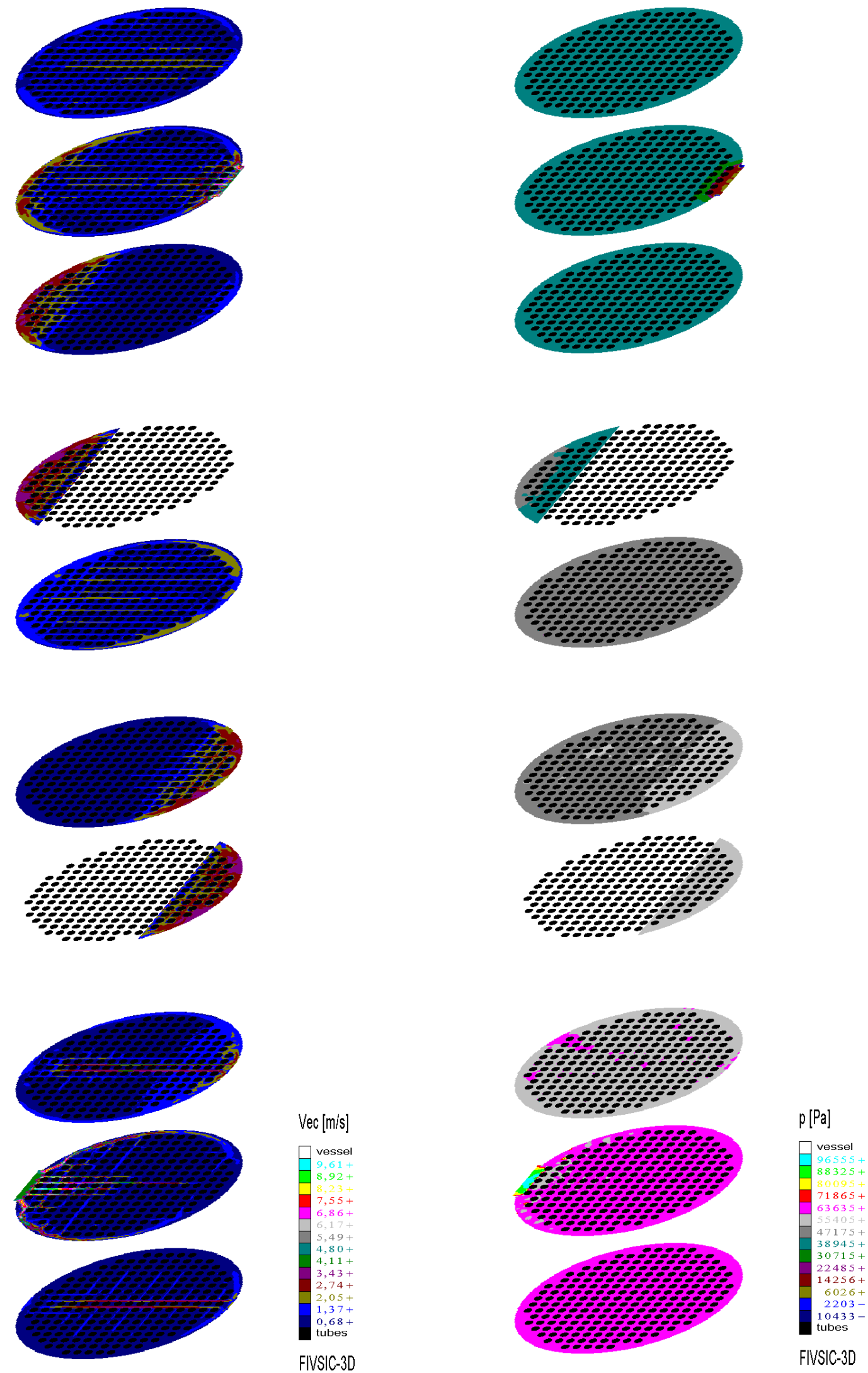

Abbildung 7-11: Geschwindigkeits- und Druckverteilung in BB1-L170 bei einem Volumenstrom von 100 m³/h. 